This document is the Accepted Manuscript version of a Published Work that appeared in final form in Organic Letters, copyright (c) American Chemical Society after peer review and technical editing by the publisher. To access the final edited and published work see http://pubs.acs.org/doi/abs/10.1021/

\title{
Pd-Catalyzed Intramolecular Oxyalkynylation of Alkenes with Hypervalent lodine
}

\author{
Stefano Nicolai, Stéphane Erard, Davinia Fernández González and \\ Jérôme Waser*
}

Laboratory of Catalysis and Organic Synthesis

Ecole Polytechnique Fédérale de Lausanne

EPFL SB ISIC LCSO, BCH 4306, 1015 Lausanne, Switzerland

jerome.waser@epfl.ch

Received Date (will be automatically inserted after manuscript is accepted)

\section{ABSTRACT}

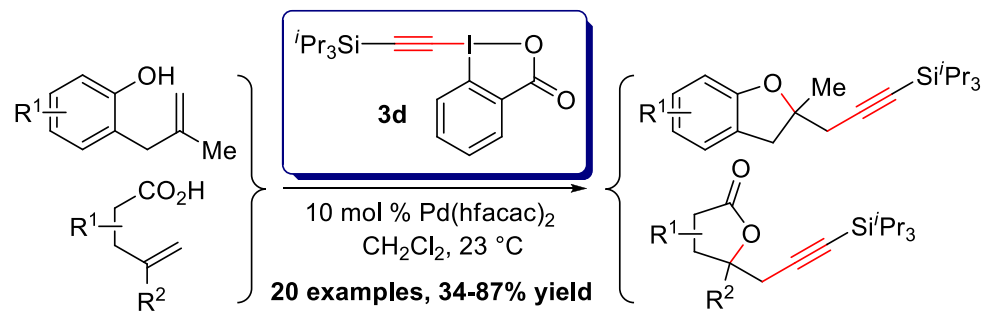

The first example of intramolecular oxyalkynylation of non-activated alkenes using oxidative Pd chemistry is reported. Both phenol and aromatic or aliphatic acid derivatives could be used under operator friendly conditions (room temperature, technical solvents, under air). The discovery of the superiority of benziodoxolone-derived hypervalent iodine reagent $3 \mathrm{~d}$ as alkyne transfer reagent further expands the rapidly increasing utility of hypervalent iodine reagents in catalysis and is expected to have important implications for other similar processes.

Cyclic structures are ubiquitous in natural products and other bioactive substances. ${ }^{1}$ Consequently, the efficient synthesis of carbo- and hetero-cycles is an important field of research in organic chemistry. Metal-catalyzed cyclization reactions, especially when involving multiple $\mathrm{C}-\mathrm{C}$ or $\mathrm{C}-\mathrm{X}$ bond formations, constitute an efficient pathway to heterocycles. ${ }^{2}$ One such method is the Wacker cyclization, which is the $\mathrm{Pd}$ catalyzed cyclization of nucleophiles on double bonds (Scheme 1, A). ${ }^{2 c, 3}$ In place of $\beta$-hydride elimination, further $\mathrm{C}-\mathrm{C},{ }^{2 \mathrm{c}, 4} \mathrm{C}-\mathrm{O},{ }^{5} \mathrm{C}-\mathrm{N}^{6}$ and $\mathrm{C}-\mathrm{Cl}^{7}$ bond formation together with cyclization have been reported. ${ }^{8}$

In particular, $\mathrm{C}-\mathrm{O}$ and $\mathrm{C}-\mathrm{N}$ bond formations have profited tremendously from the use of hypervalent iodine reagents as oxidants. ${ }^{5,6}$ In contrast, $\mathrm{C}-\mathrm{C}$ bond formation has been limited to $\mathrm{SP}^{2}$ hybridized vinyl, carbonyl and aryl groups; none of these methods reported the use of hypervalent iodine reagents. ${ }^{2 c, 4}$ It was recently demonstrated that oxidation of Pd(II) intermediates with aryliodonium salts was much slower than with $\mathrm{PhI}(\mathrm{OAc})_{2}$, which would make $\mathrm{C}-\mathrm{C}$ bond formation unable to compete with other side reactions. ${ }^{8 b}$ Herein, we report a Pd-catalyzed Wacker cyclization-alkynylation domino process using a benziodoxolone-derived reagent $\mathbf{3 d}$ (Scheme 1, B).

Acetylenes have broad utility in organic chemistry, biological chemistry and material sciences. ${ }^{9}$ Furthermore, the direct addition of acetylenes to non-activated olefins is challenging, and has been successful only for strained olefins ${ }^{10}$ or using radical methods. ${ }^{11}$ The Pd-catalyzed C$\mathrm{C}$ bond formation between a $\mathrm{SP}^{3}$ and a $\mathrm{SP}$ center is also a difficult process, which was successful only in rare cases. ${ }^{12}$ Our report constitutes the first example of intramolecular oxyalkynylation of non-activated alkenes, which represents an important breakthrough in the area of oxidative $\mathrm{Pd}$ chemistry. The discovery of the unique 
superiority of benziodoxolone derived reagents $\mathbf{3 c}-\mathbf{3 e}$ for alkynyl transfer when compared with established alkynyliodonium salts $(\mathbf{3 a}, \mathbf{3 b})$ constitutes an important advance in the burgeoning field of hypervalent iodine chemistry $^{13}$ and opens new perspective for the development of more efficient reagents in Pd-mediated C$\mathrm{C}$ bond formation and other acetylene transfer processes. Indeed, we recently discovered in our group that benziodoxolone derivatives were also unique acetylenetransfer reagents in a completely different process, namely the Au-catalyzed alkynylation of heterocycles. ${ }^{14}$

Scheme 1. Wacker Cyclization and Alkoxyalkynylation Reaction

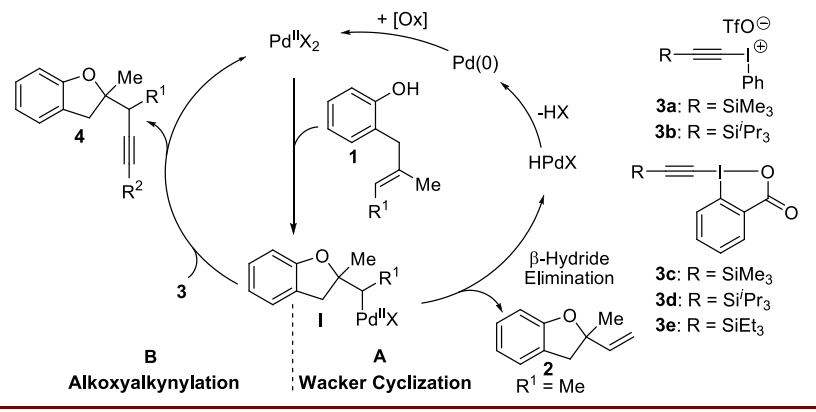

Alkynyliodonium salts are known as oxidative/electrophilic alkynylation reagents, ${ }^{13 \mathrm{c}-\mathrm{f}}$ but they have been used only rarely for the metal-mediated introduction of acetylene groups. ${ }^{15}$ Preliminary results using Stoltz' conditions ${ }^{3 \mathrm{~g}}$ with phenol $\mathbf{1 a}$ and alkynyliodonium salts 3a were disappointing, leading mostly to acetylene dimerization and low conversion (Table 1, Entry 1). However, using neutral benziodoxolone reagents $\mathbf{3 c}$ and $\mathbf{3 d}$, which have been largely ignored as acetylene transfer reagents, a 19\% yield of the desired product was obtained with 3d (Entries 2$3) .{ }^{16}$ Bases were not required and $\mathrm{CH}_{2} \mathrm{Cl}_{2}$ was the best solvent (Entry 4). ${ }^{17}$ At this point, full conversion was obtained, but a non-identified decomposition pathway consumed the starting material. ${ }^{18} \mathrm{~A}$ catalyst screen (Entries 4-6) identified $\mathrm{Pd}(\mathrm{hfacac}){ }_{2}{ }^{19}$ as the most efficient $\mathrm{Pd}$ source for preventing the decomposition of the substrates (73\% yield, Entry 6). To the best of our knowledge, the use of this complex has not been reported yet for oxidative Pd catalysis.

A sterically hindered silyl group is important to obtain good yields, (Entries 6-8), but it is the benziodoxolone structure which is essential for success, as the use of alkynyliodonium salts $\mathbf{3 a}$ or $\mathbf{3 b}$ or simple iodoacetylenes under the optimized conditions were not successful (results not shown). On a $0.40 \mathrm{mmol}$ scale with $10 \mathrm{~mol} \%$ catalyst, $71 \%$ of $\mathbf{4 b}$ was isolated after purification (Entry 9). Using technical grade $\mathrm{CH}_{2} \mathrm{Cl}_{2}$ in a flask open to air, $73 \%$ of product was obtained, demonstrating the tolerance of the reaction to air and moisture (Entry 10). On a 2.4 mmol scale, $68 \%$ of product was isolated and $65 \%$ of $2-$ iodobenzoic acid was recovered through basic extraction. This acid can then be recycled to prepare reagent $\mathbf{3 d}$ in 2 steps and $76 \%$ overall yield.

Table 1. Optimization of the Alkoxyalkynylation Reaction.

\begin{tabular}{|c|c|c|c|c|}
\hline entry & catalyst & reagent & solvent/additive & yield $^{\mathrm{a}}$ \\
\hline 1 & $\mathrm{PdCl}_{2}\left(\mathrm{CH}_{3} \mathrm{CN}\right)_{2}$ & 3a & Toluene, base ${ }^{b}$ & $6 \%(<20 \%)$ \\
\hline 2 & $\mathrm{PdCl}_{2}\left(\mathrm{CH}_{3} \mathrm{CN}\right)_{2}$ & $3 c$ & Toluene, base $\mathrm{e}^{\mathrm{b}}$ & traces $(<20 \%)$ \\
\hline 3 & $\mathrm{Pd}(\mathrm{TFA})_{2}$ & 3d & Toluene, base ${ }^{b}$ & $19 \%(73 \%)$ \\
\hline 4 & $\operatorname{Pd}(\mathrm{TFA})_{2}$ & 3d & $\mathrm{CH}_{2} \mathrm{Cl}_{2}$ & $17 \%(100 \%)$ \\
\hline 5 & $\mathrm{PdCl}_{2}\left(\mathrm{CH}_{3} \mathrm{CN}\right)_{2}$ & 3d & $\mathrm{CH}_{2} \mathrm{Cl}_{2}$ & $40 \%(93 \%)$ \\
\hline 6 & $\operatorname{Pd}(\text { hfacac })_{2}$ & 3d & $\mathrm{CH}_{2} \mathrm{Cl}_{2}$ & $73 \%(100 \%)$ \\
\hline 7 & $\operatorname{Pd}(\text { hfacac })_{2}$ & $3 c$ & $\mathrm{CH}_{2} \mathrm{Cl}_{2}$ & $20 \%(84 \%)$ \\
\hline 8 & $\operatorname{Pd}(\text { hfacac })_{2}$ & $3 e$ & $\mathrm{CH}_{2} \mathrm{Cl}_{2}$ & $43 \%^{\mathrm{c}}(>90 \%)$ \\
\hline 9 & $\operatorname{Pd}(\text { hfacac })_{2}$ & 3d & $\mathrm{CH}_{2} \mathrm{Cl}_{2}$ & $71 \% c$ \\
\hline 10 & $\operatorname{Pd}(\text { hfacac })_{2}$ & 3d & $\mathrm{CH}_{2} \mathrm{Cl}_{2}$ & $73 \% \mathrm{c,d}$ \\
\hline
\end{tabular}

aReaction conditions: $0.069 \mathrm{mmol} \mathrm{1a}, 0.014 \mathrm{mmol}$ catalyst, 0.083 mmol reagent in $5 \mathrm{~mL}$ dry solvent under $\mathrm{N}_{2}$ at $23{ }^{\circ} \mathrm{C}$ for $12-16 \mathrm{~h}$. Yield was determined via GC-MS. Conversion is given in parenthesis. b2 equiv $\mathrm{K}_{2} \mathrm{CO}_{3}$ and 0.20 equiv pyridine were used as base. cIsolated yield using $0.40 \mathrm{mmol} \mathbf{1 a}, 0.48 \mathrm{mmol} \mathbf{3 d}$ and $0.040 \mathrm{mmol}$ catalyst in $10 \mathrm{~mL}$ $\mathrm{CH}_{2} \mathrm{Cl}_{2}$. ${ }^{\mathrm{d}} \mathrm{As}$ Entry 9, but using technical solvent under air.

The scope of the reaction with phenols was examined next (Table 2, Entries 1-10). A 4-methyl group led to a lower yield (Entry 2) and more electron-rich derivatives could not be used. ${ }^{20}$ Apart from this limitation, the reaction displayed good functional group tolerance, including bromo, cyano, nitro and ketone groups (Entries 3-6). The bromo tolerance makes the method orthogonal to $\operatorname{Pd}(0)$ chemistry. The reaction was also successful for 5-Br and 6-Br substituted substrates (Entries 7 and 8). Promising preliminary results were obtained for allyl phenol 1i (Entry 9) and for the formation of 6-membered rings (Entry 10). The former result demonstrates that alkynylation can be efficient even in the presence of a $\beta$ hydrogen atom. Although full conversion was observed, no compound was isolated in significant amount beside the desired product and optimization of the reaction conditions was not successful to improve the yield.

As we hypothesized, the limitation of the scope observed with phenols was due to the sensibility of the substrate under oxidative conditions, we thus decided to examine benzoic acid derivatives next (Table 1, Entries 11-15). 
Table 2. Scope of the Oxyalkynylation reaction.

\begin{tabular}{|c|c|c|c|}
\hline entry & substrate & product & isolated yielda \\
\hline 1 & 1a: $\mathrm{R}=\mathrm{H}$ & $4 b$ & $71 \%$ \\
\hline 2 & 1b: $\mathrm{R}=4-\mathrm{Me}$ & 4d & $34 \%$ \\
\hline 3 & 1c: $\mathrm{R}=4-\mathrm{Br}$ & $4 e$ & $68 \%$ \\
\hline 4 & 1d: $\mathrm{R}=4-\mathrm{CN}$ & $4 f$ & $76 \%$ \\
\hline 5 & 1e: $\mathrm{R}=4-\mathrm{NO}_{2}$ & $4 \mathrm{~g}$ & $72 \%$ \\
\hline 6 & 1f: $R=4-A c$ & $4 h$ & $87 \%$ \\
\hline 7 & $1 \mathrm{~g}: \mathrm{R}=5-\mathrm{Br}$ & $4 \mathbf{i}$ & $66 \%$ \\
\hline 8 & 1h: $\mathrm{R}=6-\mathrm{Br}$ & $4 \mathbf{j}$ & $52 \%$ \\
\hline 9 & & & $43 \%{ }^{b}$ \\
\hline 10 & & & $46 \% \mathrm{c}$ \\
\hline 11 & & & $83 \%$ \\
\hline 12 & & & $80 \%$ \\
\hline 13 & & & $70 \%$ \\
\hline 14 & & & $56 \%$ \\
\hline 15 & & & $80 \%$ \\
\hline 16 & $\mathrm{Me}$ & $8 \mathbf{b}: X=$ & $\begin{array}{c}69 \% \\
\mathbf{8 a}: \mathbf{8 b}=1.5: 1\end{array}$ \\
\hline 17 & & & $70 \%{ }^{\mathrm{d}}$ \\
\hline 18 & $9 b$ & & $72 \% \mathrm{~d}$ \\
\hline 19 & & & $76 \%{ }^{d}$ \\
\hline 20 & & & $82 \%{ }^{d}$ \\
\hline
\end{tabular}

aReaction conditions: $0.40 \mathrm{mmol}$ substrate, $0.48 \mathrm{mmol}$ reagent and $0.040 \mathrm{mmol} \mathrm{Pd}(\mathrm{hfacac})_{2}$ in $10 \mathrm{~mL} \mathrm{CH}_{2} \mathrm{Cl}_{2}$ at $23{ }^{\circ} \mathrm{C}$ for $12 \mathrm{~h}$. bIn $10 \mathrm{~mL}$ $\mathrm{CHCl}_{3}$. ${ }^{\mathrm{N}} \mathrm{No}$ full conversion was observed. ${ }^{\mathrm{d}}$ Reaction time was $3 \mathrm{~h}$.

Indeed, excellent results were obtained and an electrondonating group on the benzene ring (Entry 12) or a diaryl olefin (Entry 13) were now tolerated in the reaction. Even a trisubstituted olefin could be used (Entry 14). In this case the 1,3 addition product was obtained, probably via isomerization of the formed Pd-alkyl intermediate. Formation of a 6-membered ring was also possible in $80 \%$ yield (Entry 15). A single example of nitrogen nucleophile gave a useful amount of product, but further control of the chemoselectivity will be needed (Entry 16).

Although aliphatic alcohols could not be used (not shown), aliphatic acids worked well in the reaction and full conversion and good yields were obtained after $3 \mathrm{~h}$ (Entries 17-20). In this case, the presence of $\beta$-hydrogen on the double bond was not deleterious to the yield (Entries 19-20) and even simple commercially available 4-pentenoic acid could be cyclized in good yield (Entry 20). The obtained $\gamma$-lactones bearing an acetylene group are important building blocks for the synthesis of bioactive compounds. $^{21}$

As the oxyalkynylation process involves several bond forming steps, understanding the mechanism is challenging. Nucleophilic attack on alkynyliodonium salts proceeds via $\beta$-addition followed by 1,2 shift. ${ }^{13 \mathrm{~d}}$ However, when a ${ }^{13} \mathrm{C}$-label was introduced next to $\mathrm{Si}$ in 3d, no shift was observed (eq 1), eliminating the possibility of this pathway. At least two mechanisms could still be envisaged (Scheme 2): (1) oxidative addition of $\mathbf{3 d}$ to $\mathrm{Pd}^{\mathrm{II}}$ to form a $\mathrm{Pd}^{\mathrm{IV}}$ intermediate $\mathbf{I I},{ }^{22}$ followed by oxy-palladation to form $\mathbf{V}$ and reductive elimination to give $\mathbf{4 a}$ or (2) initial oxy-palladation to give IV, either via phenolate complex III or directly from 1a; oxidative reaction with $\mathbf{3 d}$ to form $\mathbf{V}$ and reductive elimination. Currently, we tend to favor the latter, as oxidation to form a $\mathrm{Pd}^{\mathrm{IV}}$ from $\mathrm{Pd}^{\mathrm{II}}$-alkyl intermediate should be easier, electron-deficient catalysts worked best and the intramolecular oxy-palladation of olefins with $\mathrm{Pd}^{\mathrm{II}}$ catalysts is well-precedented. ${ }^{3 \mathrm{~g}}$ Furthermore, a fast reaction was not observed when mixing the reagent and the catalyst.

Scheme 2. Speculative Mechanism.

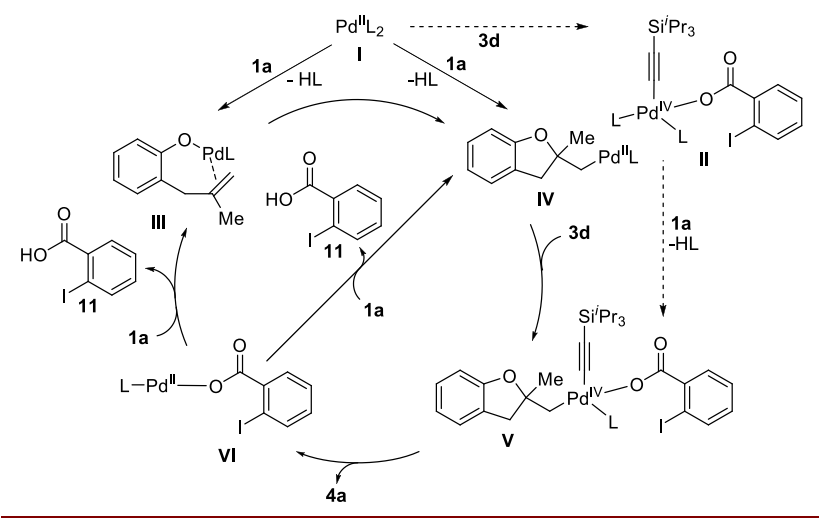




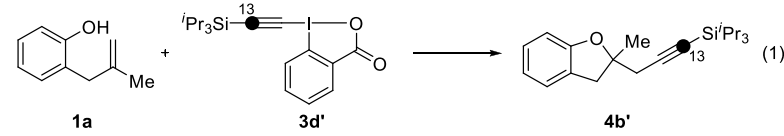

Finally, we think that the basicity of the 2-iodo benzoate formed in the reaction could be important to promote reaction turnover by accelerating proton transfer from 1a in the oxy-palladation step (conversion of VI to III or IV in Scheme 2). The generation of a basic carboxylate upon reaction is again specific to the benziodoxolone derivative 3d, and could further contribute to the exceptional performance of this reagent when compared with more often used alkynyliodonium salts.

In summary, we have reported a novel oxyalkynylation reaction of olefins. Our work represents the first example of acetylene incorporation to olefins using oxidative $\mathrm{Pd}$ chemistry. It is operatively simple and was successful both in the case of phenol and acid derivatives. With phenols, good yields were obtained only for substrates with a geminally disubstituted double bond and electrondonating groups on the benzene ring were not tolerated. With acids, the scope was more general, and good yields were obtained, even for the monosubstituted double bond and in the case of aliphatic substrates. The unprecedented use of benziodoxolone-derived hypervalent iodine was essential for success. Further work on the reaction mechanism, an asymmetric method and the use of hypervalent iodine reagents in other catalytic alkynylation reactions is currently ongoing in our group.

Acknowledgment EPFL and SNF (grant number 200021_119810) are acknowledged for financial support, Dr. Tom Woods (Chemical Synthesis Laboratory, EPFL) for proofreading and Dr. Laure Menin and Mr. Francisco Sepulveda (Mass Spectrometry Services, ISIC, EPFL) for HIRES mass spectra.

Supporting Information Available Experimental details and characterization data. This material is available free of charge via the Internet at http://pubs.acs.org.

\section{References}

(1) Clardy, J.; Walsh, C. Nature 2004, 432, 829

(2) (a) Tietze, L. F. Chem. Rev. 1996, 96, 115. (b) Nakamura, I.; Yamamoto, Y. Chem. Rev. 2004, 104, 2127. (c) Zeni, G.; Larock, R. C. Chem. Rev. 2004, 104, 2285. (d) Zeni, G.; Larock, R. C. Chem. Rev. 2006, 106, 4644.

(3) Pioneering works: (a) Hosokawa, T.; Hirata, M.; Murahashi, S. I.; Sonoda, A. Tetrahedron Lett. 1976, 1821. (b) Hegedus, L. S. Allen, G. F.; Bozell, J. J.; Waterman, E. L. J. Am. Chem. Soc. 1978, 100, 5800. Recent examples: (c) Uozumi, Y.; Kato, K.; Hayashi, T. J. Am. Chem. Soc. 1997, 119, 5063. (d) Arai, M. A.; Kuraishi, M.; Arai, T.; Sasai, H. J. Am. Chem. Soc. 2001, 123 , 2907. (e) Fix, S. R.; Brice, J. L.; Stahl, S. S. Angew. Chem., Int Ed. 2002, 41, 164. (f) Kotov, V.; Scarborough, C. C.; Stahl, S. S. Inorg. Chem. 2007, 46, 1910. (g) Trend, R. M.; Ramtohul, Y. K.; Stoltz, B. M. J. Am. Chem. Soc. 2005, 127, 17778.

(4) (a) Hegedus, L. S.; Allen, G. F.; Olsen, D. J. J. Am. Chem. Soc. 1980, 102, 3583. (b) Tietze, L. F.; Sommer, K. M.; Zinngrebe, J.; Stecker, F. Angew. Chem., Int. Ed. 2005, 44, 257. (c) Rosewall, C. F.; Sibbald, P. A.; Liskin, D. V.; Michael, F. E. J. Am. Chem. Soc. 2009, 131, 9488. (d) Cernak, T. A.; Lambert, T. H. J. Am. Chem. Soc. 2009, 131, 3124. Domino-Heck C-X bond formation: (e)
Wolfe, J. P.; Rossi, M. A. J. Am. Chem. Soc. 2004, 126, 1620. (f) Wolfe, J. P. Synlett 2008, 2913.

(5) (a) Alexanian, E. J.; Lee, C.; Sorensen, E. J. J. Am. Chem. Soc. 2005, 127, 7690. (b) Desai, L. V.; Sanford, M. S. Angew. Chem. Int. Ed. 2007, 46, 5737. (c) Li, Y.; Song, D.; Dong, V. M. J. Am. Chem. Soc. 2008, 130, 2962.

(6) (b) Muniz, K. J. Am. Chem. Soc. 2007, 129, 14542. (c) Muniz, K.; Hovelmann, C. H.; Streuff, J. J. Am. Chem. Soc. 2008, 130, 763. (d) Sibbald, P. A.; Michael, F. E. Org. Lett. 2009, 11, 1147.

(7) (a) Manzoni, M. R.; Zabawa, T. P.; Kasi, D.; Chemler, S. R. Organometallics 2004, 23, 5618. (b) Michael, F. E.; Sibbald, P. A.; Cochran, B. M. Org. Lett. 2008, 10, 793.

(8) Review: (a) Beccalli, E. M.; Broggini, G.; Martinelli, M.; Sottocornola, S. Chem. Rev. 2007, 107, 5318. For a discussion of high oxidation states of Pd, see: (b) Deprez, N. R.; Sanford, M. S. J. Am. Chem. Soc. 2009, 131, 11234. (c) Powers, D. C.; Ritter, T. Nature Chem. 2009, 1, 302.

(9) Diederich F.; Stang, P. J.; Tykwinski, R. R.; Acetylene Chemistry Chemistry, Biology and Material Science; Wiley-VCH, Weinheim, 2005.

(10) (a) Shirakura, M.; Suginome, M. J. Am. Chem. Soc. 2008, 130, 5410. (b) Shirakura, M.; Suginome, M. J. Am. Chem. Soc. 2009, 131, 5060. (c) Kohno, K.; Nakagawa, K.; Yahagi, T.; Choi, J. C.; Yasuda, H.; Sakakura, T. J. Am. Chem. Soc. 2009, 131, 2784.

(11) (a) Gong, J. C.; Fuchs, P. L. J. Am. Chem. Soc. 1996, 118, 4486. (b) Schaffner, A. P.; Darmency, V.; Renaud, P. Angew. Chem., Int Ed. 2006, 45, 5847.

(12) Altenhoff, G.; Wurtz, S.; Glorius, F. Tetrahedron Lett. 2006, 47, 2925.

(13) (a) Wirth, T. Hypervalent iodine chemistry: modern developments in organic synthesis, Vol. 224, Springer, New York, 2003. (b) Zhdankin, V. V.; Stang, P. J. Chem. Rev. 2008, 108, 5299. (c) Beringer, F. M.; Galton, S. A. J. Org. Chem. 1965, 30, 1930. (d) Ochiai, M.; Ito, T.; Takaoka, Y.; Masaki, Y.; Kunishima, M. Tani, S.; Nagao, Y. J. Chem. Soc. Chem. Commun. 1990, 118. (e) Stang, P. J.; Arif, A. M.; Crittell, C. M. Angew. Chem., Int. Ed. Engl. 1990, 29, 287. (f) Zhdankin, V. V.; Stang, P. J. Tetrahedron 1998, 54, 10927. (g) Ochiai, M.; Masaki, Y.; Shiro, M. J. Org. Chem. 1991, 56, 5511. (h) Zhdankin, V. V.; Kuehl, C. J.; Krasutsky, A. P.; Bolz, J. T.; Simonsen, A. J. J. Org. Chem. 1996, $61,6547$.

(14) Brand, J. B.; Charpentier, J.; Waser, J. Angew. Chem., Int. Ed. 2009, 48, 9346.

(15) (a) Kang, S. K.; Lee, H. W.; Jang, S. B.; Ho, P. S. J. Org. Chem. 1996, 61, 4720. (b) Huang, X. A.; Zhong, P. J. Chem. Soc. Perkin Trans. 1 1999, 1543. (c) Yu, C. M.; Kweon, J. H.; Ho, P. S.; Kang, S. C.; Lee, G. Y. Synlett 2005, 2631. (d) Canty, A. J.; Rodemann, T.; Skelton, B. W.; White, A. H. Organometallics 2006, 25, 3996. (e) Canty, A. J.; Gardiner, M. G.; Jones, R. C.; Rodemann, T.; Sharma, M. J. Am. Chem. Soc. 2009, 131, 7236. (f) Chaudhuri, P. D.; Guo, R.; Malinakova, H. C. J. Organomet. Chem. 2008, 693, 567.

(16) Previous to our work, the only report of benziodoxolone reagents for $\mathrm{C}-\mathrm{C}$ bond formation concerned $\mathrm{CF}_{3}$-transfer: (a) Eisenberger, P.; Gischig, S.; Togni, A. Chem. Eur. J. 2006, 12, 2579. (b) Kieltsch, I.; Eisenberger, P.; Togni, A. Angew. Chem., Int. Ed. 2007, 46, 754. (c) Koller, R.; Stanek, K.; Stolz, D.; Aardoom, R.; Niedermann, K.; Togni, A. Angew. Chem., Int. Ed. 2009, 48, 4332.

(17) See Supporting Information for a complete list of tested reaction conditions and catalysts.

(18) Beside the desired product, no low-molecular weight compound was isolated from the reaction mixture. That would indicate that the main pathway for decomposition is polymerization, either via the alkene, or via the aromatic nucleus, for example through hypervalent iodine mediated oxidation.

(19) hfacac $=$ hexafluoroacetonate.

(20) Excepted when stated otherwise, full conversion was observed for all substrates in table 2. In case of electron-rich substrates, decomposition was observed and no defined low-molecular weight product could be isolated. We speculate that this class of substrates is not compatible with the oxidative conditions of the reaction.

(21) (a) Jones, E. R. H.; Jones, J. B.; Skattebol, L.; Whiting, M. C. J. Chem. Soc. 1960, 3489. (b) Doad, G. J. S.; Barltrop, J. A.; Petty, C. M.; Owen, T. C. Tetrahedron Lett. 1989, 30, 1597. (c) Braukmuller, S.; Bruckner, R. Eur. J. Org. Chem. 2006, 2110. (d) Habel, A.; Boland, W. Org. Biomol. Chem. 2008, 6, 1601.

(22) See reference $15 \mathrm{~d}$ for an example of $\mathrm{Pd}^{\mathrm{IV}}$-acetylene complex. 
Supporting information

\section{Pd-Catalyzed Intramolecular Oxyalkynylation of Alkenes with Hypervalent Iodine}

Stefano Nicolai, Stéphane Erard, Davinia Fernández González and Jérôme Waser

Laboratory of Catalysis and Organic Synthesis, Ecole Polytechnique Fédérale de Lausanne, EPFL SB ISIC LCSO, BCH 4306,
1015 Lausanne, Switzerland

jerome.waser@epfl.ch

81 Pages

1. General Methods

2. Preparation of Reagents

3. Preparation of Substrates

4. Optimization of the Reaction

5. Scope of the Reaction

6. Mechanistic Investigations

7. Spectra of New Compounds p. S2

p. S2

p. S7

p. S22

p. S25

p. S35

p. S41 


\section{General Methods}

All reactions were carried out in oven dried glassware under an atmosphere of nitrogen, unless stated otherwise. For quantitative flash chromatography technical grade solvents were used. For flash chromatography for analysis, HPLC grade solvents from Sigma-Aldrich were used. THF, $\mathrm{Et}_{2} \mathrm{O}, \mathrm{CH}_{3} \mathrm{CN}$, toluene, hexane and $\mathrm{CH}_{2} \mathrm{Cl}_{2}$ were dried by passage over activated alumina under nitrogen atmosphere $\left(\mathrm{H}_{2} \mathrm{O}\right.$ content $<30 \mathrm{ppm}$, KarlFischer titration). $\mathrm{NEt}_{3}$ and pyridine were distilled under nitrogen from $\mathrm{KOH}$. All chemicals were purchased from Acros, Aldrich, Fluka, VWR, Aplichem or Merck and used as such unless stated otherwise. Chromatographic purification was performed as flash chromatography using Macherey-Nagel silica 40-63, 60 $\AA$, using the solvents indicated as eluent with 0.1-0.5 bar pressure. TLC was performed on Merck silica gel 60 F254 TLC glass plates or aluminium plates and visualized with UV light, permanganate stain, CAN stain or Anisaldehyde stain. Melting points were measured on a Büchi B-540 melting point apparatus using open glass

capillaries, the data is uncorrected. ${ }^{1} \mathrm{H}-\mathrm{NMR}$ spectra were recorded on a Brucker DPX-400 $400 \mathrm{MHz}$ spectrometer in chloroform-d, DMSO- $\mathrm{d}_{6}, \mathrm{CD}_{2} \mathrm{Cl}_{2}$ or $\mathrm{CD}_{3} \mathrm{OD}$, all signals are reported in ppm with the internal chloroform signal at $7.26 \mathrm{ppm}$, the internal DMSO signal at $2.50 \mathrm{ppm}$, the internal $\mathrm{CD}_{2} \mathrm{Cl}_{2}$ signal at $5.31 \mathrm{ppm}$, or the internal methanol signal at $3.30 \mathrm{ppm}$ as standard. The data is being reported as $(\mathrm{s}=\operatorname{singlet}, \mathrm{d}=\mathrm{doublet}, \mathrm{t}$ = triplet, $\mathrm{q}=$ quadruplet, $\mathrm{qi}=$ quintet, $\mathrm{m}=$ multiplet or unresolved, $\mathrm{b}=$ broad signal, coupling constant $(\mathrm{s})$ in $\mathrm{Hz}$, integration; interpretation). ${ }^{13} \mathrm{C}$-NMR spectra were recorded with ${ }^{1} \mathrm{H}$-decoupling on a Brucker DPX-400 100 $\mathrm{MHz}$ spectrometer in chloroform-d, DMSO- $\mathrm{d}_{6}, \mathrm{CD}_{2} \mathrm{Cl}_{2}$ or $\mathrm{CD}_{3} \mathrm{OD}$, all signals are reported in ppm with the internal chloroform signal at $77.0 \mathrm{ppm}$, the internal DMSO signal at $39.5 \mathrm{ppm}$, the internal $\mathrm{CD}_{2} \mathrm{Cl}_{2}$ signal at $53.5 \mathrm{ppm}$ or the internal methanol signal at $49.0 \mathrm{ppm}$ as standard. Infrared spectra were recorded on a JASCO FT-IR B4100 spectrophotometer with an ATR PRO410-S and a ZnSe prisma and are reported as $\mathrm{cm}^{-1}(\mathrm{w}=$ weak, $\mathrm{m}=$ medium, $\mathrm{s}=$ strong, $\mathrm{sh}=$ shoulder). Gas chromatographic and low resolution mass spectrometric measurements were performed on a Perkin-Elmer Clarus 600 gas chromatographer and mass spectrometer using a Perkin-Elmer Elite fused silica column (length: $30 \mathrm{~m}$, diameter: $0.32 \mathrm{~mm}$ ) and Helium as carrier gas. High resolution mass spectrometric measurements were performed by the mass spectrometry service of ISIC at the EPFL on a MICROMASS (ESI) Q-TOF Ultima API. HPLC measurement were done on a JASCO HPLC system with an AS2055 Autosampler, a PU 2089 Pump, a UV 2075 detector and a SEDEX 85 (SEDERE) detector using a CHIRALPAK IC column from DAICEL Chemical Industries Ltd. HPLC grade solvents from Sigma-Aldrich were used.

\section{Preparation of Reagents}

Phenyl(trimethylsilylethynyl)iodonium triflate (3a). 


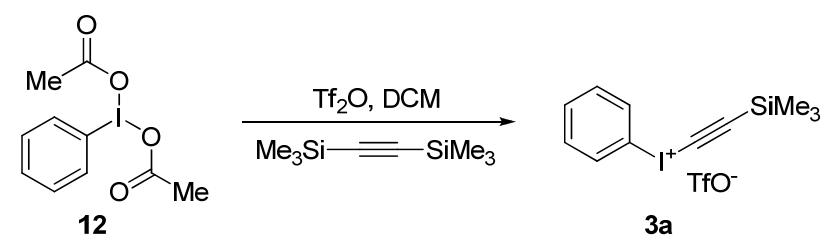

Following a reported procedure, ${ }^{1}$ phenyliodonium diacetate (12) $(3.22 \mathrm{~g}, 10.0 \mathrm{mmol}, 1.00$ equiv) was diluted with $\mathrm{CH}_{2} \mathrm{Cl}_{2}(10 \mathrm{~mL})$ and the mixture was stirred for 5 minutes. $\mathrm{Tf}_{2} \mathrm{O}(0.67 \mathrm{~mL}, 5.0 \mathrm{mmol}, 0.50$ equiv. $)$ was added dropwise at $0{ }^{\circ} \mathrm{C}$ and the resulting yellow mixture was stirred $30 \mathrm{~min}$. Bis(trimethylsilyl)acetylene ( $2.28 \mathrm{~mL}, 10.0 \mathrm{mmol}, 1.00$ equiv) was added. The mixture was then stirred $2 \mathrm{~h}$ and diethyl ether was added to precipitate the product. Filtration afforded $3 a(2.11 \mathrm{~g}, 4.67 \mathrm{mmol}, 47 \%$ yield) as a colorless solid. Mp (Dec.) 139-145 ${ }^{\circ}$ C; Lit.: ${ }^{[1]} 143-146^{\circ} \mathrm{C} .{ }^{1} \mathrm{H}$ NMR $\left(400 \mathrm{MHz}, \mathrm{CDCl}_{3}\right) \delta 8.07$ (d, $\left.J=8.3 \mathrm{~Hz}, 2 \mathrm{H}, \mathrm{ArH}\right), 7.66(\mathrm{~s}, 1 \mathrm{H}$, ArH), 7.55 (m, 2 H, ArH), 0.24 (s, 9 H, TMS). $\left.{ }^{13} \mathrm{C} \mathrm{NMR} \mathrm{(100} \mathrm{MHz,} \mathrm{CDCl}_{3}\right) \delta 133.9,132.4,132.2,119.7$ (q, $J=$ 319 Hz), 119.1, 116.2, 43.3, -1.1. IR v 1448 (w), 1286 (m), 1253 (m), 1236 (s), 1222 (s), 1161 (m), $1026(\mathrm{~s})$, $988(\mathrm{w}), 863(\mathrm{~m}), 847(\mathrm{~s}), 742(\mathrm{w}), 714(\mathrm{~m}), 678(\mathrm{w}), 637$ (s). Characterization data of 3a correspond to the literature values. ${ }^{1}$

\section{Phenyl(triisopropylsilyl)iodonium triflate (3b)}

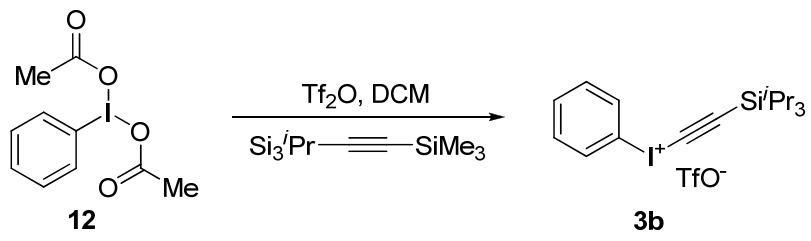

Following a slight modification of the reported procedure, ${ }^{1}$ phenyliodonium diacetate (12) $(2.53 \mathrm{~g}, 7.85$ mmol, 1.00 equiv) was diluted with $\mathrm{CH}_{2} \mathrm{Cl}_{2}(7 \mathrm{~mL})$ and the mixture was stirred for 5 minutes. $\mathrm{Tf}_{2} \mathrm{O}(0.60 \mathrm{~mL}$, 3.9 mmol, 0.50 equiv.) was added dropwise at $0{ }^{\circ} \mathrm{C}$ and the resulting yellow mixture was stirred 30 min. (Trimethylsilyl)(triisopropylsilyl)acetylene $(2.00 \mathrm{~g}, 7.86 \mathrm{mmol}, 1.00$ equiv) was added and the mixture was then stirred $2 \mathrm{~h}$. Water was then added $(30 \mathrm{~mL})$ followed by extraction of the aqueous layer with $\mathrm{CH}_{2} \mathrm{Cl}_{2}(2 \times 30$ $\mathrm{mL}$ ). The combined organic layers were dried over $\mathrm{MgSO}_{4}$, filtered and the solvent was evaporated under reduced pressure. The resulting solid was triturated in hexane $(10 \mathrm{~mL})$. Filtration and removal of solvent in vacuo afforded phenyl(triisopropylsilyl)iodonium triflate (3b) $(2.90 \mathrm{~g}, 11.2 \mathrm{mmol}, 70 \%$ yield) as a colorless solid. Mp 109-114 ${ }^{\circ} \mathrm{C} .{ }^{1} \mathrm{H}$ NMR $\left(400 \mathrm{MHz}, \mathrm{CDCl}_{3}\right) \delta 8.09$ (m, $\left.2 \mathrm{H}, \mathrm{Ar} H\right), 7.65$ (m, $\left.1 \mathrm{H}, \mathrm{Ar} H\right), 7.52(\mathrm{~m}, 2 \mathrm{H}$, $\mathrm{Ar} H), 1.15-1.01$ (m, $21 \mathrm{H}, \mathrm{TIPS}) .{ }^{13} \mathrm{C}$ NMR $\left(100 \mathrm{MHz}, \mathrm{CDCl}_{3}\right) \delta 133.7,132.5,132.4,119.7,117.6,117.6,44.9$, 18.3, 11.1. IR v 3288 (w), 3088 (m), 2949 (m), 2894 (m), 2869 (w), 1563 (m), 1467 (w), 1451 (w), 1388 (w), 1281 (s), 1236 (s), 1221 (s), 1174 (s), 1068 (w), 1028 (s), 988 (m), 916 (m), 884 (m), 736 (s), 679 (m), 639 (s). HRMS (ESI) calcd for $\mathrm{C}_{17} \mathrm{H}_{26} \mathrm{ISi}^{+}$(M-OTf) 385.0843; found 385.0812.

\section{1-Hydroxy-1,2-benziodoxol-3(1H)-one (13)}




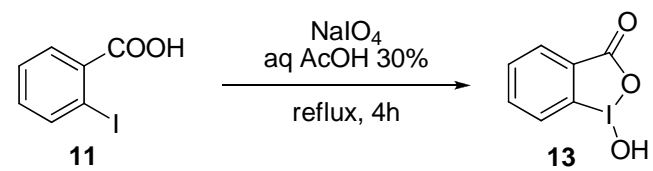

Following a reported procedure, ${ }^{2} \mathrm{NaIO}_{4}(6.7 \mathrm{~g}, 31 \mathrm{mmol} ; 1.0$ equiv) and 2-iodobenzoic acid (11) (7.4 g, 30 mmol, 1.0 equiv) were suspended in $30 \%(\mathrm{v}: \mathrm{v})$ aq. AcOH $(45 \mathrm{~mL})$. The mixture was vigorously stirred and refluxed for $4 \mathrm{~h}$. The reaction mixture was then diluted with cold water $(120 \mathrm{~mL})$ and allowed to cool to room temperature, protecting it from light. After $1 \mathrm{~h}$, the crude product was collected by filtration, washed on the filter with ice water $(3 \times 30 \mathrm{~mL})$ and acetone $(3 \times 30 \mathrm{~mL})$, and air-dried in the dark to give the pure product 13 (7.3 g, $19 \mathrm{mmol}, 92 \%$ yield) as a colorless solid. ${ }^{1} \mathrm{H}$ NMR (400 MHz, $\left.\left(\mathrm{CD}_{3}\right)_{2} \mathrm{SO}\right) \delta 8.02(\mathrm{dd}, J=7.7,1.4 \mathrm{~Hz}, 1$ $\mathrm{H}, \operatorname{Ar} H), 7.97(\mathrm{~m}, 1 \mathrm{H}, \operatorname{Ar} H), 7.85(\mathrm{dd}, J=8.2,0.7 \mathrm{~Hz}, 1 \mathrm{H}, \mathrm{Ar} H), 7.71(\mathrm{td}, J=7.6,1.2 \mathrm{~Hz}, 1 \mathrm{H}, \operatorname{Ar} H) .{ }^{13} \mathrm{C}$ NMR (100 MHz, $\left.\left(\mathrm{CD}_{3}\right)_{2} \mathrm{SO}\right) \delta 167.7,134.5,131.5,131.1,130.4,126.3,120.4 . \mathrm{IR} v 3083(\mathrm{w}), 3060(\mathrm{w}), 2867$ (w), 2402 (w), 1601 (m), 1585 (m), 1564 (m), 1440 (m), 1338 (s), 1302 (m), 1148 (m), $1018(\mathrm{w}), 834(\mathrm{~m}), 798$ (w), $740(\mathrm{~s}), 694(\mathrm{~s}), 674(\mathrm{~m}), 649(\mathrm{~m})$. The characterization data for compounds 13 correspond to the reported values. $^{2}$

\section{1-[(Trimethylsilyl)ethynyl]-1,2-benziodoxol-3(1H)-one (3c)}

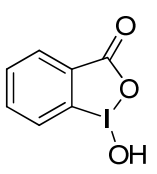

13

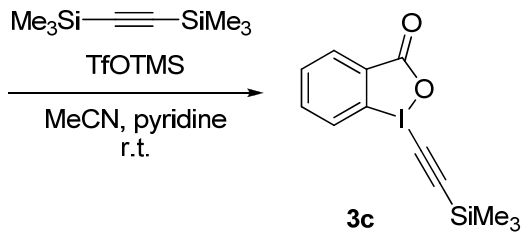

Following a reported procedure, ${ }^{3}$ trimethylsiyltriflate $(2.8 \mathrm{~mL}, 15 \mathrm{mmol}, 1.4$ equiv, freshly distilled $)$ was added dropwise to a stirred solution of 2-iodosylbenzoic acid (13) (3.00 g, $11.4 \mathrm{mmol}, 1.00$ equiv) in acetonitrile $(85 \mathrm{~mL})$ until the mixture turned colorless. Bis(trimethylsilyl)acetylene $(2.14 \mathrm{~g}, 12.5 \mathrm{mmol}, 1.10$ equiv) was then added dropwise, followed, after $20 \mathrm{~min}$, by the addition of pyridine (1.2 $\mathrm{mL}, 15 \mathrm{mmol}, 1.4$ equiv). The mixture was stirred $30 \mathrm{~min}$. The solvent was then removed under reduced pressure and the yellow crude oil was dissolved in dichloromethane $(80 \mathrm{~mL})$. The organic layer was washed with a large amount of water $(130 \mathrm{~mL})$, and the aqueous layer was extracted with $\mathrm{CH}_{2} \mathrm{Cl}_{2}(3 \times 65 \mathrm{~mL})$. The organic layer was washed with brine $(130 \mathrm{~mL})$, dried over $\mathrm{MgSO}_{4}$, filtered and the solvent was evaporated under reduced pressure. Recrystallization from acetonitrile $(2.3 \mathrm{~mL})$ afforded 3c $(2.35 \mathrm{~g}, 6.84 \mathrm{mmol}, 60 \%$ yield $)$ as a colorless solid. . Mp: $143-145^{\circ} \mathrm{C}(\mathrm{dec}) .{ }^{1} \mathrm{H}$ NMR $\left(400 \mathrm{MHz}, \mathrm{CDCl}_{3}\right) \delta 8.42(\mathrm{dd}, J=6.4,1.9 \mathrm{~Hz}, 1 \mathrm{H}, \mathrm{Ar} H), 8.19(\mathrm{~m}, 1 \mathrm{H}, \mathrm{Ar} H)$, 7.78 (m, $2 \mathrm{H}, \mathrm{ArH}), 0.32$ (s, $9 \mathrm{H}, \mathrm{TMS}) .{ }^{13} \mathrm{C} \mathrm{NMR}\left(100 \mathrm{MHz}, \mathrm{CDCl}_{3}\right)$ 166.4, 134.9, 132.6, 131.7, 131.4, 126.1, 117.2, 115.4, 64.2, -0.5. IR v 3389 (w), 2967 (w), 1617 (s), 1609 (s), 1562 (m), 1440 (w), 1350 (m), 1304 (w), $1254(\mathrm{w}), 1246(\mathrm{w}), 1112(\mathrm{w}), 1008(\mathrm{w}), 852(\mathrm{~s}), 746(\mathrm{~m}), 698(\mathrm{~m}), 639(\mathrm{~m})$. The characterization data for compounds $3 c$ corresponded to the reported values. ${ }^{3}$

(2) Kraszkiewicz, L.; Skulski, L. Arkivoc, 2003, 6, 120.

(3) Zhdankin, V. V.; Kuehl, C. J.; Krasutsky, A. P.; Bolz, J. T.; Simonsen, A. J. J. Org. Chem. 1996, 61, 6547. 


\section{Triisopropylsilyl trimethylsilylacetylene (15)}

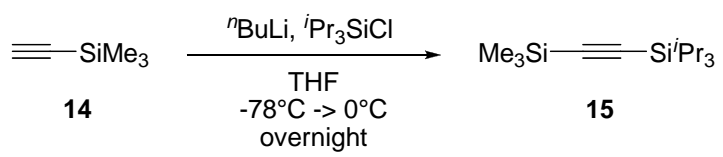

Following a reported procedure, ${ }^{4} n$-butyllithium $(2.5 \mathrm{M}$ in hexanes, $12.0 \mathrm{~mL}, 29.9 \mathrm{mmol}, 0.98$ equiv) was added dropwise to a stirred solution of ethynyltrimethylsilane (14) (3.0 g, $30 \mathrm{mmol}, 1.0$ equiv) in THF (48 mL) at $-78{ }^{\circ} \mathrm{C}$. The mixture was then warmed to $0{ }^{\circ} \mathrm{C}$ and stirred for $5 \mathrm{~min}$. The mixture was then cooled back to -78 ${ }^{\circ} \mathrm{C}$ and chlorotriisopropylsilane $(6.4 \mathrm{~mL}, 30 \mathrm{mmol}, 1.0$ equiv) was added dropwise. The mixture was then allowed to warm to room temperature and stirred overnight. A saturated solution of ammonium chloride (40 $\mathrm{mL}$ ) was added, and the reaction mixture was extracted with diethyl ether $(2 \mathrm{x} 60 \mathrm{~mL})$. The organic layer was washed with water and brine, then dried over $\mathrm{MgSO}_{4}$, filtered and concentrated under reduced pressure to obtain a colorless liquid which was further purified by Kugelrohr distillation $\left(56-57^{\circ} \mathrm{C} / 0.25 \mathrm{mmHg}\right)$ to yield 15 (7.16 g, $28.0 \mathrm{mmol}, 92 \%$ yield) as a colorless liquid. ${ }^{1} \mathrm{H}$ NMR (400 MHz, $\left.\mathrm{CDCl}_{3}\right) \delta 1.08$ (m, $\left.21 \mathrm{H}, \mathrm{TIPS}\right), 0.18$ (s, 9 H, TMS). IR v 2959 (m), 2944 (m), 2896 (w), 2867 (m), 1464 (w), 1385 (w), 1250 (m), 996 (w), 842 (s), $764(\mathrm{~s}), 675(\mathrm{~m}), 660(\mathrm{~m})$. Characterization data of 15 correspond to the literature values. ${ }^{4}$

\section{1-[(Triisopropyllsilyl)ethynyl]-1,2-benziodoxol-3(1H)-one (3d)}

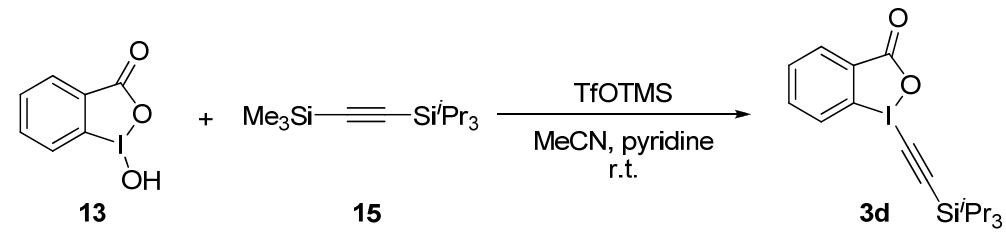

Following a reported procedure, ${ }^{3}$ trimethylsilyltriflate $(3.6 \mathrm{~mL}, 20 \mathrm{mmol}, 1.1$ equiv, freshly distilled) was added dropwise to a stirred solution of 2-iodosylbenzoic acid (13) (4.7 g, $18 \mathrm{mmol}, 1.0$ equiv) in acetonitrile $(140 \mathrm{~mL})$. (Trimethylsilyl)(triisopropylsilyl)acetylene (15) $(5.0 \mathrm{~g}, 20 \mathrm{mmol}, 1.1 \mathrm{equiv})$ was then added dropwise, followed, after $15 \mathrm{~min}$, by the addition of pyridine (1.5 mL, $20 \mathrm{mmol}, 1.1 \mathrm{equiv})$. The mixture was stirred $10 \mathrm{~min}$. The solvent was then removed under reduced pressure and the yellow crude oil was dissolved in dichloromethane $(50 \mathrm{~mL})$. The organic layer was washed with $\mathrm{HCl} 1 \mathrm{M}(50 \mathrm{~mL})$ and the aqueous layer was extracted with $\mathrm{CH}_{2} \mathrm{Cl}_{2}(50 \mathrm{~mL})$. The organic layers were combined, washed with a saturated solution of $\mathrm{NaHCO}_{3}(2 * 50 \mathrm{~mL})$, dried over $\mathrm{MgSO}_{4}$, filtered and the solvent was evaporated under reduced pressure. Recrystallization from acetonitrile ( $c$ a $35 \mathrm{~mL})$ afforded $3 \mathbf{d}(6.3 \mathrm{~g}, 15 \mathrm{mmol}, 83 \%)$ as a colorless solid. Mp (Dec.) $170-176^{\circ} \mathrm{C} .{ }^{1} \mathrm{H}$ NMR $\left(400 \mathrm{MHz}, \mathrm{CDCl}_{3}\right) \delta 8.44(\mathrm{~m}, 1 \mathrm{H}, \mathrm{ArH}), 8.29$ (m, $\left.1 \mathrm{H}, \mathrm{ArH}\right), 7.77$ (m, $\left.2 \mathrm{H}, \mathrm{ArH}\right)$, 1.16 (m, $21 \mathrm{H}, \mathrm{TIPS}) .{ }^{13} \mathrm{C} \mathrm{NMR}\left(100 \mathrm{MHz}, \mathrm{CDCl}_{3}\right) \delta 166.4,134.6,132.3,131.4,131.4,126.1,115.6,114.1$, 64.6, 18.4, 11.1. IR v $2943(\mathrm{~m}), 2865$ (m), $1716(\mathrm{~m}), 1618(\mathrm{~m}), 1604$ (s), 1584 (m), 1557 (m), 1465 (m), 1439

(4) Helal, C J.; Magriotis, P. A.; Corey, E. J. J. Am. Chem. Soc. 1996, 118, 10938. 
(w), 1349 (m), 1291 (m), 1270 (w), 1244 (m), 1140 (m), 1016 (m), 999 (m), 883 (m), 833 (m), 742 (m), 702 (s), $636(\mathrm{~m})$. Characterization data of $\mathbf{3 d}$ corresponded to the literature values. ${ }^{3}$

Triethyl trimethylsilylacetylene (16)

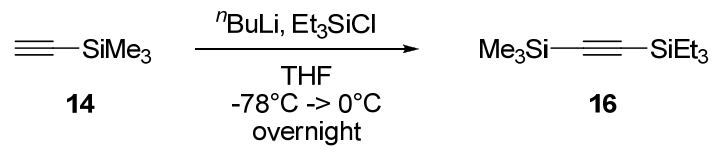

Following a reported procedure ${ }^{4} n$-butyllithium $(2.5 \mathrm{M}$ in hexanes, $5.4 \mathrm{~mL}, 14 \mathrm{mmol}, 1.0$ equiv) was added dropwise to a stirred solution of ethynyltrimethylsilane (14) (1.36 g, $13.8 \mathrm{mmol}, 1.00$ equiv) in THF (21 mL) at $-78{ }^{\circ} \mathrm{C}$. The mixture was then warmed to $0{ }^{\circ} \mathrm{C}$ and stirred for $5 \mathrm{~min}$. The mixture was then cooled back to -78 ${ }^{\circ} \mathrm{C}$ and chlorotriethylsilane $(2.3 \mathrm{~mL}, 14 \mathrm{mmol}, 0.98$ equiv) was added dropwise. The mixture was then allowed to warm to room temperature and stirred overnight. A saturated solution of ammonium chloride $(20 \mathrm{~mL}) \mathrm{was}$ added, and the reaction mixture was extracted with diethyl ether $(2 \times 20 \mathrm{~mL})$. The organic layer was washed with water and brine, then dried over $\mathrm{MgSO}_{4}$, filtered and concentrated under reduced pressure to obtain a colorless liquid which was further purified by Kugelrohr distillation to yield 16 (3.4 g, $11 \mathrm{mmol}, 83 \%$ yield) as a colorless liquid. ${ }^{1} \mathrm{H}$ NMR $\left(400 \mathrm{MHz}, \mathrm{CDCl}_{3}\right) \delta 0.99\left(\mathrm{t}, J=7.9 \mathrm{~Hz}, 9 \mathrm{H}, \mathrm{SiCH}_{2} \mathrm{CH}_{3}\right), 0.59$ (q, J=7.9 Hz, $6 \mathrm{H}$, $\mathrm{SiCH}_{2} \mathrm{CH}_{3}$ ), 0.17 (s, $\left.9 \mathrm{H}, \mathrm{TMS}\right) .{ }^{13} \mathrm{C} \mathrm{NMR}\left(100 \mathrm{MHz}, \mathrm{CDCl}_{3}\right) \oint 115.4,111.2,7.4,4.4,0.0 . \mathrm{IR} v 2958$ (m), 2913 (m), $2879(\mathrm{~m}), 1462(\mathrm{w}), 1414(\mathrm{w}), 1381(\mathrm{w}), 1250(\mathrm{~m}), 1015(\mathrm{~m}), 973(\mathrm{w}), 908(\mathrm{w}), 844(\mathrm{~s}), 773(\mathrm{~s}), 731(\mathrm{~s})$ $702(\mathrm{sh}), 679(\mathrm{sh})$.

\section{1-[(Triethylsilyl)ethynyl]-1,2-benziodoxol-3(1H)-one (3e)}

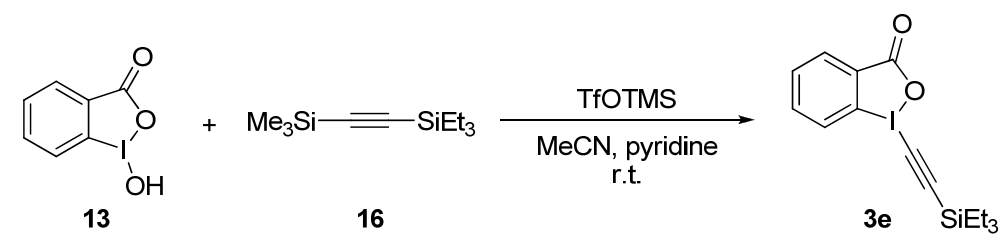

Following a reported procedure, ${ }^{3}$ trimethylsilyltriflate $(1.2 \mathrm{~mL}, 6.3 \mathrm{mmol}, 1.4$ equiv, freshly distilled) was added dropwise to a stirred solution of 2-iodosylbenzoic acid (13) (1.2 g, $4.5 \mathrm{mmol}, 1.0$ equiv) in acetonitrile $(33 \mathrm{~mL})$ until the mixture turned colorless. The mixture was cooled to $0^{\circ} \mathrm{C}$ and (Trimethylsilyl)(triethylsilyl)acetylene (16) (1.0 g, $4.9 \mathrm{mmol}, 1.1$ equiv) was then added dropwise. The reaction mixture was stirred at $0^{\circ} \mathrm{C}$ for $1 \mathrm{~h}$ and then allowed to warm to room temperature. After 20 min pyridine ( 0.50 $\mathrm{mL}, 6.4 \mathrm{mmol}, 1.4$ equiv) was added and the mixture was stirred for an additional $30 \mathrm{~min}$. The solvent was then removed under reduced pressure and the yellow crude oil was dissolved in dichloromethane $(50 \mathrm{~mL})$. The organic layer was washed with a large amount of water $(80 \mathrm{~mL})$, and the aqueous layer was extracted with $\mathrm{CH}_{2} \mathrm{Cl}_{2}(3 \mathrm{x} 40 \mathrm{~mL})$. The organic layer was washed with brine $(80 \mathrm{~mL})$, dried over $\mathrm{MgSO}_{4}$, filtered and the solvent was evaporated under reduced pressure. Recrystallization from acetonitrile $(1.5 \mathrm{~mL})$ afforded $3 \mathbf{e}(0.30$ g, $0.80 \mathrm{mmol}, 17 \%$ yield) as a colorless solid. Mp (Dec.) $111-130^{\circ} \mathrm{C} .{ }^{1} \mathrm{H}$ NMR $\left(400 \mathrm{MHz}, \mathrm{CDCl}_{3}\right) \delta 8.40(\mathrm{~m}$, 
$1 \mathrm{H}, \mathrm{Ar} H), 8.24(\mathrm{~m}, 1 \mathrm{H}, \mathrm{Ar} H), 7.75(\mathrm{~m}, 2 \mathrm{H}, \mathrm{ArH}), 1.06\left(\mathrm{t}, J=8.0 \mathrm{~Hz}, 9 \mathrm{H}, \mathrm{SiCH}_{2} \mathrm{CH}_{3}\right), 0.73$ (q, $J=8.0 \mathrm{~Hz}$ $\left.6 \mathrm{H}, \mathrm{SiCH}_{2} \mathrm{CH}_{3}\right) .{ }^{13} \mathrm{C} \mathrm{NMR}\left(100 \mathrm{MHz}, \mathrm{CDCl}_{3}\right) \delta 166.5,134.8,132.5,131.6,131.3,126.1,115.5,115.1,64.6$, 7.4, 4.1. IR v 3064 (w), 3062 (m), 2957 (m), 2911 (m), 2877 (m), 1621 (s), 1587 (m), 1561 (m), 1460 (m), 1440 (m), 1415 (w), 1378 (w), 1336 (m), 1297 (m), 1237 (w), 1149 (w), 1113 (w), 1010 (m), 976 (w), 912 (w), 912 (w), $834(\mathrm{~m}), 804(\mathrm{w}), 739$ (s), 693 (m), 675 (m), 647 (w). HRMS (ESI) calcd for $\mathrm{C}_{15} \mathrm{H}_{20} \mathrm{IO}_{2} \mathrm{Si}^{+}(\mathrm{M}+\mathrm{H})$ 387.0277; found 387.0290.

\section{2-Iodo-1-triisopropylsilyl acetylene (18)}

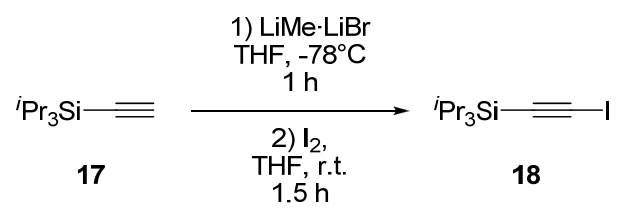

Following a reported procedure, ${ }^{5} \mathrm{MeLi} \cdot \mathrm{LiBr}(1.5 \mathrm{M}$ in diethyl ether, 1.1.mL, $1.6 \mathrm{mmol}, 1.0$ equiv) was added to a stirred solution of triisopropylsilylacetylene $(17)(0.36 \mathrm{~mL}, 1.6 \mathrm{mmol}, 1.0$ equiv) in dry THF (1.8 mL), cooled at $-78^{\circ} \mathrm{C}$, and the mixture was allowed to react for $1 \mathrm{~h}$ at that temperature. A solution of $\mathrm{I}_{2}(457 \mathrm{mg}, 1.80$ mmol, 1.25 equiv) in dry THF $(2.7 \mathrm{~mL})$ was then added dropwise and the mixture was stirred for $1.5 \mathrm{~h}$ at $-78^{\circ} \mathrm{C}$. The mixture was then diluted with brine $(6 \mathrm{~mL})$ and the aqueous layer was extracted with ether $(3 \mathrm{x} 10 \mathrm{~mL})$. The combined organic layers were washed with a saturated aqueous solution of $\mathrm{Na}_{2} \mathrm{~S}_{2} \mathrm{O}_{3}(3 \times 20 \mathrm{~mL})$, dried over $\mathrm{MgSO}_{4}$ and concentrated under reduced pressure. Purification by column chromatography $\left(\mathrm{SiO}_{2}\right.$, hexane) afforded 2-iodo-1-triisopropylsilyl acetylene (18) (470 mg, $1.52 \mathrm{mmol}, 94 \%$ yield) as a colorless oil. ${ }^{1} \mathrm{H}$ NMR $\left(400 \mathrm{MHz} \mathrm{CDCl}_{3}\right) \delta 1.10-1.04$ (m, $\left.21 \mathrm{H}, \mathrm{TIPS}\right) .{ }^{13} \mathrm{C} \mathrm{NMR}\left(100 \mathrm{MHz}, \mathrm{CDCl}_{3}\right) \delta 100.8,18.5,11.4$ (one acetylene carbon was not resolved). Characterization data of $\mathbf{1 8}$ correspond to the literature values. ${ }^{5}$

\section{Preparation of Substrates}

\section{2-(Methylallyl)phenol (1a)}

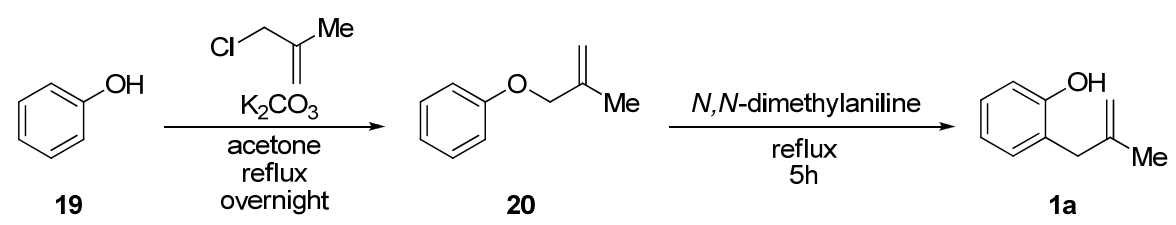

Following a reported procedure, ${ }^{6}$ potassium carbonate $(3.0 \mathrm{~g}, 22 \mathrm{mmol}, 1.1$ equiv) and methyl allyl chloride (2.15 mL, $22.0 \mathrm{mmol}, 1.10$ equiv) were added to a stirred solution of phenol (19) (1.9 g, $20 \mathrm{mmol}, 1.0$ equiv) in dry acetone $(5.0 \mathrm{~mL})$. The mixture was refluxed overnight. Then it was allowed to cool to room temperature and water $(25 \mathrm{~mL})$ was added, followed by extraction with ether $(3 \times 10 \mathrm{~mL})$. The combined organic layers

(5) López, S.; Fernández-Trillo, F.; Midón, P.; Castedo, L.; Saá, L. J. Org. Chem., 2005, 70, 6346.

(6) Goering, H. L.; Jacobson, R. R. J. Am. Chem. Soc. 1958, 80, 3277. 
were then washed with $2 \mathrm{M} \mathrm{NaOH}(3 \times 20 \mathrm{~mL})$, dried with $\mathrm{MgSO}_{4}$, filtered and concentrated in vacuo to afford 1-(2-methylallyloxy)benzene (20) $(1.2 \mathrm{~g}, \leq 8.1 \mathrm{mmol}, \leq 41 \%$ yield) which was used without further purification.

1-(2-Methylallyloxy)benzene (20) (1.2 g, $8.1 \mathrm{mmol}, 1.0$ equiv) was dissolved in $N, N$-dimethylaniline (1.0 $\mathrm{mL}, 8.1 \mathrm{mmol}, 1.0$ equiv) and the mixture was refluxed $5 \mathrm{~h}$ at $205{ }^{\circ} \mathrm{C}$ under $\mathrm{N}_{2}$. It was then cooled to RT and diethyl ether $(100 \mathrm{~mL})$ was added and the reaction mixture washed with $1 \mathrm{M} \mathrm{HCl}(3 \times 50 \mathrm{~mL})$. The aqueous layer was further extracted with diethyl ether $(3 \times 50 \mathrm{~mL})$. The organic layer was washed with water and brine. Then it was dried over $\mathrm{MgSO}_{4}$ and concentrated under reduced pressure. The crude product was purified by flash chromatography $\left(\mathrm{SiO}_{2}\right.$, PET/EtOAc 30:1-5:1) to yield 1a (0.357 g, $24.1 \mathrm{mmol}, 30 \%$ yield) as a yellow oil. $\mathrm{R}_{f}$ 0.36, (PET/EtOAc 10:1). ${ }^{1} \mathrm{H}$ NMR (400 MHz, $\left.\mathrm{CDCl}_{3}\right) \delta 7.17(\mathrm{td}, J=7.7,1.3 \mathrm{~Hz}, 1 \mathrm{H}, \mathrm{Ar} H), 7.13(\mathrm{~d}, J=7.4$ $\mathrm{Hz}, 1 \mathrm{H}, \mathrm{ArH}), 6.91(\mathrm{t}, J=7.7 \mathrm{~Hz}, 1 \mathrm{H}, \mathrm{ArH}), 6.86(\mathrm{~d}, J=8.0 \mathrm{~Hz}, 1 \mathrm{H}, \mathrm{ArH}), 5.34(\mathrm{~d}, J=1.6 \mathrm{~Hz}, 1 \mathrm{H}, \mathrm{OH}), 4.95$ $\left(\mathrm{s}, 1 \mathrm{H}, \mathrm{C}=\mathrm{CH}_{2}\right), 4.87\left(\mathrm{~s}, 1 \mathrm{H}, \mathrm{C}=\mathrm{CH}_{2}\right), 3.41\left(\mathrm{~s}, 2 \mathrm{H}, \mathrm{CH}_{2}\right), 1.77\left(\mathrm{~s}, 3 \mathrm{H}, \mathrm{CH}_{3}\right) .{ }^{13} \mathrm{C} \mathrm{NMR}\left(100 \mathrm{MHz} \mathrm{CDCl}_{3}\right) \delta$ 154.6, 144.6, 130.9, 127.9, 124.8, 120.7, 116.0, 112.3, 39.7, 22.1. IR v $3461(\mathrm{~m}), 3072(\mathrm{w}), 3034(\mathrm{w}), 2971(\mathrm{w})$, $2915(\mathrm{w}), 1650(\mathrm{w}), 1591(\mathrm{w}), 1489$ (m), $1456(\mathrm{~s}), 1375(\mathrm{w}), 1337$ (w), 1331 (w), $1256(\mathrm{~m}), 1227$ (m), 1214 (m), $1171(\mathrm{~m}), 1094(\mathrm{~m}), 1042(\mathrm{w}), 893(\mathrm{~m}), 856(\mathrm{w}), 753(\mathrm{~s}), 721(\mathrm{w})$. Characterization data corresponded to the literature values. ${ }^{6}$

\section{4-Methyl-2(2-methylallyl)phenol (1b)}

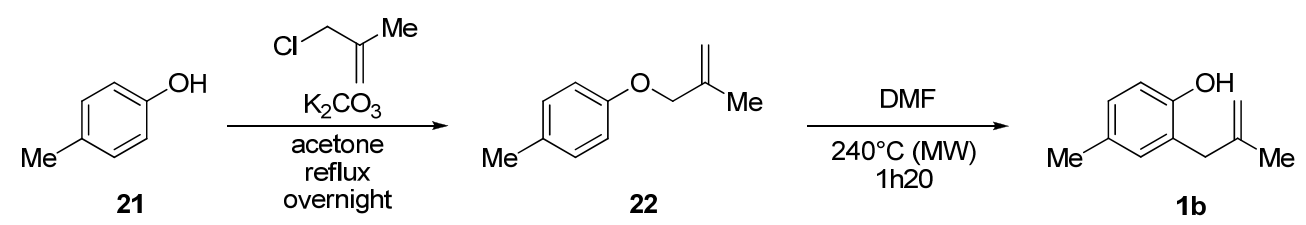

Following a slight modification of the reported procedure, ${ }^{6}$ potassium carbonate $(3.3 \mathrm{~g}, 24 \mathrm{mmol}, 1.2$ equiv) and methyl allyl chloride $(2.4 \mathrm{~mL}, 24 \mathrm{mmol}, 1.2$ equiv) were added to a stirred solution of $p$-cresol (21) (2.2 g, $20 \mathrm{mmol}, 1.0$ equiv) in dry acetone $(5.0 \mathrm{~mL})$. The mixture was stirred under reflux overnight. Then it was allowed to cool to room temperature and water $(25 \mathrm{~mL})$ was added, followed by extraction with ether $(3 \mathrm{x} 10$ $\mathrm{mL})$. The combined organic layers were then washed with $2 \mathrm{M} \mathrm{NaOH}(3 \times 20 \mathrm{~mL})$ and dried with $\mathrm{MgSO}_{4}$, filtered and concentrated in vacuo to afford 1-methyl-4-(2-methylallyloxy)benzene (22) (2.02 g, $\leq 12.5 \mathrm{mmol}, \leq$ $61 \%$ yield) which was used without further purification.

1-Methyl-4-(2-methylallyloxy)benzene (22) (0.80 g, $4.9 \mathrm{mmol})$ was dissolved in DMF (4.0 $\mathrm{mL})$ and the mixture was stirred under $\mathrm{N}_{2}$ at $240^{\circ} \mathrm{C}$ under microwave irradiation for $1 \mathrm{~h} 20$. DMF was evaporated in vacuo. The resulting crude product was purified by chromatography $\left(\mathrm{SiO}_{2}\right.$, PET/EtOAc 30:1-15:1) to yield 1b (404 mg, 2.49 mmol, 51\% yield) as a yellow oil. $\mathrm{R}_{f} 0.63$ (PET/EtOAc 10:1). ${ }^{1} \mathrm{H}$ NMR $\left(400 \mathrm{MHz}, \mathrm{CDCl}_{3}\right) \delta 6.94(\mathrm{dd}$, $J=8.1,1.7 \mathrm{~Hz}, 1 \mathrm{H}, \operatorname{Ar} H), 6.90(\mathrm{~m}, 1 \mathrm{H}, \operatorname{ArH}), 6.73(\mathrm{~d}, J=8.1 \mathrm{~Hz}, 1 \mathrm{H}, \operatorname{ArH}), 4.98(\mathrm{~s}, 1 \mathrm{H}, \mathrm{OH}), 4.92$ (s, $1 \mathrm{H}$, $\left.\mathrm{C}=\mathrm{CH}_{2}\right), 4.85\left(\mathrm{~s}, 1 \mathrm{H}, \mathrm{C}=\mathrm{CH}_{2}\right), 3.34\left(\mathrm{~s}, 2 \mathrm{H}, \mathrm{CH}_{2}\right), 2.27(\mathrm{~s}, 3 \mathrm{H}, \mathrm{PhCH}), 1.75\left(\mathrm{~s}, 3 \mathrm{H}, \mathrm{CH}_{3}\right) .{ }^{13} \mathrm{C} \mathrm{NMR}(100$ $\left.\mathrm{MHz}, \mathrm{CDCl}_{3}\right) \delta 152.2,144.7,131.4,129.8,128.3,124.6,115.8,112.1,39.7,22.1,20.4 . \mathrm{IR} v 3427$ (broad, m), 
3173 (w), 3075 (w), 3015 (m), 2971 (m), 2918 (m), 2863 (m), 1650 (w), 1614 (w), 1504 (s), 1442 (m), 1375 (m), 1337 (m), 1260 (s), 1229 (s), 1196 (s), 1150 (m), 1105 (s), 1040 (w), 1021 (w), 933 (sh), 890 (s), 812 (s), $782(\mathrm{w}), 748(\mathrm{w}), 716(\mathrm{w}), 696(\mathrm{w}), 695(\mathrm{w}), 683(\mathrm{w}), 664(\mathrm{w}), 652(\mathrm{w}), 634(\mathrm{w}), 620(\mathrm{w})$. Characterization data corresponded to the literature values. ${ }^{7}$

\section{4-Methoxy-2(2-methylallyl)phenol (25)}

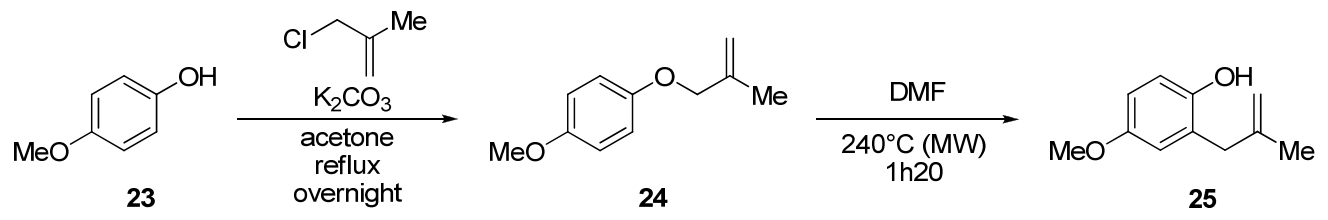

Following a slight modification of the reported procedure, ${ }^{6}$ potassium carbonate $(2.9 \mathrm{~g}, 21 \mathrm{mmol}, 1.3 \mathrm{equiv})$ and methyl allyl chloride $(2.0 \mathrm{~mL}, 21 \mathrm{mmol}, 1.3$ equiv) were added to a stirred solution of $p$-methoxy phenol (23) $(2.0 \mathrm{~g}, 16 \mathrm{mmol}, 1.0$ equiv) in dry acetone $(4.0 \mathrm{~mL})$. The mixture was stirred under reflux overnight. Then it was allowed to cool to room temperature and water $(25 \mathrm{~mL})$ was added, followed by extraction with ether $(3$ x $10 \mathrm{~mL}$ ). The combined organic layers were then washed with $2 \mathrm{M} \mathrm{NaOH}(3 \times 20 \mathrm{~mL})$, dried with $\mathrm{MgSO}_{4}$, filtered and concentrated in vacuo to afford 1-methyl-4-(2-methylallyloxy)benzene (24) $(2.01 \mathrm{~g}, \leq 11.3 \mathrm{mmol}, \leq$ $70 \%$ yield), which was used without further purification.

1-Methyl-4-(2-methylallyloxy)benzene (24) (0.85 g, $4.8 \mathrm{mmol})$ was dissolved in DMF (3.8 $\mathrm{mL})$ and the mixture was stirred under $\mathrm{N}_{2}$ at $240^{\circ} \mathrm{C}$ under microwave irradiation for $1 \mathrm{~h} 20$. DMF was evaporated in vacuo. The resulting crude product was purified by chromatography $\left(\mathrm{SiO}_{2}, \mathrm{PET} /\right.$ EtOAc 30:1-15:1) to yield 25 (684 $\mathrm{mg}, 3.84 \mathrm{mmol}, 80 \%$ yield) as a yellow oil. $\mathrm{R}_{f} 0.23$ (PET/EtOAc 10:1). ${ }^{1} \mathrm{H} \mathrm{NMR}\left(400 \mathrm{MHz}, \mathrm{CDCl}_{3}\right) \delta 6.77(\mathrm{~m}$, $1 \mathrm{H}, \mathrm{ArH}), 6.72-6.65(\mathrm{~m}, 2 \mathrm{H}, \mathrm{ArH}), 4.92(\mathrm{bs}, 1 \mathrm{H}, \mathrm{OH}), 4.87-4.82\left(\mathrm{~m}, 2 \mathrm{H}, \mathrm{C}=\mathrm{CH}_{2}\right), 3.75(\mathrm{~s}, 3 \mathrm{H}, \mathrm{OCH})$, $3.34\left(\mathrm{~s}, 2 \mathrm{H}, \mathrm{CH}_{2}\right), 1.75$ (s, $\left.3 \mathrm{H}, \mathrm{CH}_{3}\right) .{ }^{13} \mathrm{C} \mathrm{NMR}\left(100 \mathrm{MHz}, \mathrm{CDCl}_{3}\right) \oint 153.5,148.6,144.5,126.5,116.6,116.5$, 112.7, 112.2, 55.8, 39.5, 22.2. IR v 3436 (sh), 3436 (m), 3384 (sh), 3076 (w), 3033 (w), $2971(w), 2938$ (w), $2911(\mathrm{w}), 2911$ (w), 2835 (w), 1650 (w), 1611 (w), 1502 (s), 1432 (s), 1374 (m), 1344 (m), 1343 (w), 1274 (w), 1254 (s), 1205 (sh), 1182 (s), 1152 (w), 1106 (m), 1042 (s), 892 (m), 806 (m), 739 (s), 714 (sh), 666 (w), 633 (w), $609(w)$. Characterization data corresponded to the literature values. ${ }^{8}$

\section{4-Bromo-2-(2-methylallyl)phenol (1c)}

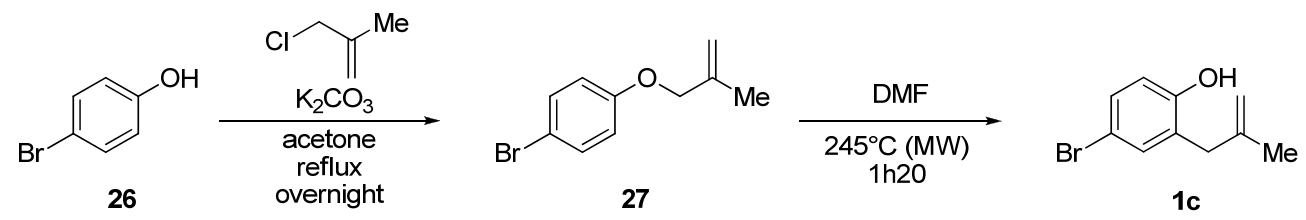

(7) Summermatter, W.; Heimgartner, H. Helvetica Chimica Acta 1984, 67, 1298.

(8) Mali, R. S.; Garkhedkar, M. P.; Sindkhedkar, M. D.; Dhavale, D. D. J. Chem. Res., Synopses 1996, 7, 342. 
Following a slight modification of the reported procedure, ${ }^{6}$ potassium carbonate $(2.4 \mathrm{~g}, 17 \mathrm{mmol}, 1.2$ equiv $)$ and methyl allyl chloride (1.7 mL, $17 \mathrm{mmol}, 1.2$ equiv) were added to a stirred solution of 4-bromo phenol (26) (2.5 g, $14 \mathrm{mmol}, 1.0$ equiv) in dry acetone $(5.0 \mathrm{~mL})$. The mixture was stirred under reflux overnight. Then it was allowed to cool to room temperature and water $(25 \mathrm{~mL})$ was added, followed by extraction with ether $(3 \mathrm{x}$ $10 \mathrm{~mL})$. The combined organic layers were then washed with $2 \mathrm{M} \mathrm{NaOH}(3 \times 20 \mathrm{~mL})$, dried with $\mathrm{MgSO}_{4}$, filtered and concentrated in vacuo to afford 1-bromo-4-(2-methylallyloxy)benzene (27) $(2.84 \mathrm{~g}, \leq 12.5 \mathrm{mmol}, \leq$ $87 \%$ yield) which was used without further purification.

1-Methyl-4-(2-methylallyloxy)benzene (27) (1.1 g, $4.8 \mathrm{mmol})$ was dissolved in DMF (4.0 $\mathrm{mL})$ and the mixture was stirred under $\mathrm{N}_{2}$ at $245^{\circ} \mathrm{C}$ under microwave irradiation for $1 \mathrm{~h} 20$. DMF was evaporated in vacuo. The resulting crude product was purified by chromatography $\left(\mathrm{SiO}_{2}, \mathrm{PET} /\right.$ EtOAc $\left.20: 1-15: 1\right)$ to yield 1c $(0.86 \mathrm{~g}$, $3.8 \mathrm{mmol}, 80 \%$ yield) as a yellow oil. $\mathrm{R}_{f}=0.43$, (PET/EtOAc 10:1). ${ }^{1} \mathrm{H}$ NMR $\left(400 \mathrm{MHz}, \mathrm{CDCl}_{3}\right) \delta 7.31-7.21$ $(\mathrm{m}, 2 \mathrm{H}, \mathrm{ArH}), 6.74(\mathrm{~d}, J=8.2 \mathrm{~Hz}, 1 \mathrm{H}, \mathrm{ArH}), 5.22(\mathrm{~m}, 1 \mathrm{H}, \mathrm{OH}), 4.97\left(\mathrm{~s}, 1 \mathrm{H}, \mathrm{C}=\mathrm{CH}_{2}\right), 4.89\left(\mathrm{~m}, 1 \mathrm{H}, \mathrm{C}=\mathrm{CH}_{2}\right)$, $3.36\left(\mathrm{~s}, 2 \mathrm{H}, \mathrm{CH}_{2}\right), 1.76\left(\mathrm{~s}, 3 \mathrm{H}, \mathrm{CH}_{3}\right) .{ }^{13} \mathrm{C} \mathrm{NMR}\left(100 \mathrm{MHz}, \mathrm{CDCl}_{3}\right) \delta 153.7,143.9,133.4,130.7,127.4,117.8$, 113.0, 112.6, 39.4, 22.1; IR v 3450 (broad), 3071 (w), 2972 (w), 2914 (w), 2851 (w), 1650 (m), 1603 (w),1584 (w), $1480(\mathrm{~s}), 1420$ (m),1411(s), 1376 (m), 1323 (m), 1266 (s), 1211 (s), 1168 (s), 1107 (s), 1020 (w), 891 (s), 809 (s), 779 (w), 672 (m). HRMS (ESI) ${ }^{9}$ expected for $\mathrm{C}_{10} \mathrm{H}_{11}{ }^{79} \mathrm{BrO}^{107} \mathrm{Ag}^{+}\left(\mathrm{M}+{ }^{107} \mathrm{Ag}\right)$ 332.9035; found 332.8976 .

\section{4-Hydoxy-3-(2-methylallyl)benzonitrile (1d)}

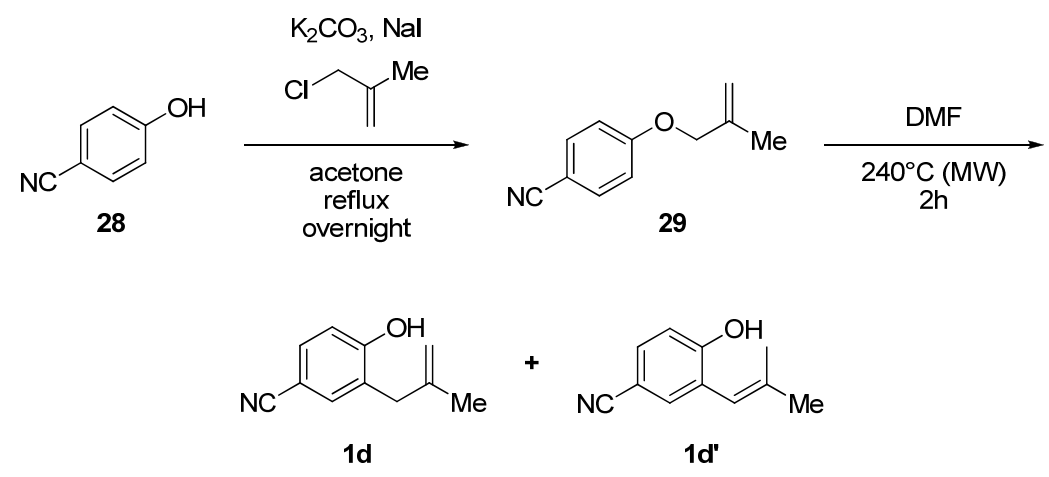

Following a slight modification of the reported procedure, ${ }^{10}$ potassium carbonate $(1.5 \mathrm{~g}, 11 \mathrm{mmol}, 1.3$ equiv), methyl allyl chloride (1.3 mL, $13 \mathrm{mmol}, 1.5$ equiv) and $\mathrm{NaI}$ (tip of a spatula) were added to a stirred solution of 4-hydroxy benzonitrile (28) (1.0 g, $8.5 \mathrm{mmol}, 1.0$ equiv) in dry acetone $(5.2 \mathrm{~mL})$. The mixture was stirred under reflux overnight. Then it was allowed to cool to room temperature and water $(25 \mathrm{~mL})$ was added, followed by extraction with ether $(3 \times 10 \mathrm{~mL})$. The combined organic layers were then washed with $2 \mathrm{M} \mathrm{NaOH}$

(9) ESI mass spectrum of compound 1c was acquired after treatment of 1c with $\mathrm{AgNO}_{3}$, as a consequence of troublesome ionization of the pure compound.

(10) Stanetty, P.; Koller, H.; Pürstinger, G. Monatsh. Chem. 1990, 121, 883. 
(3 $\mathrm{x} 20 \mathrm{~mL}$ ). It was dried with $\mathrm{MgSO}_{4}$, filtered and concentrated in vacuo to afford 4-(2methylallyloxy)benzonitrile (29) $(1.4 \mathrm{~g}, \leq 8.1 \mathrm{mmol}, \leq 95 \%$ yield $)$ which was used without further purification.

4-(2-Methylallyloxy)benzonitrile (29) $(0.52 \mathrm{~g}, 3.0 \mathrm{mmol})$ was dissolved in DMF $(2.4 \mathrm{~mL})$ and the mixture was stirred under $\mathrm{N}_{2}$ at $240{ }^{\circ} \mathrm{C}$ under microwave irradiation for $2 \mathrm{~h}$. DMF was evaporated in vacuo. The resulting crude product was purified by chromatography $\left(\mathrm{SiO}_{2}\right.$, PET/EtOAc 20:1-5:1) to yield a yellowish solid (237 mg; mixture of inseparable isomers 1d, 1d'. Based on ${ }^{1} \mathrm{H}$ NMR, 1d was the most abundant isomer, the ratio being 4.5:1; 1d: $194 \mathrm{mg}, 1.12 \mathrm{mmol}, 37 \%$ yield. The mixture was used in the following reaction without separation, assuming 1d' as a non reactive isomer). $\mathrm{R}_{f}=0.33,(\mathrm{PET} / \mathrm{EtOAc} 4: 1) .{ }^{1} \mathrm{H} \mathrm{NMR}\left(400 \mathrm{MHz}, \mathrm{CDCl}_{3}\right) \delta$ $7.45(\mathrm{dd}, J=2.0,8.0 \mathrm{~Hz}, 1 \mathrm{H}, \mathrm{ArH}), 7.42(\mathrm{~d}, J=2.0 \mathrm{~Hz}, 1 \mathrm{H}, \operatorname{ArH}), 7.36(d, J=2.0 \mathrm{~Hz}), 6.96(d, J=8.5 \mathrm{~Hz})$, $6.89(\mathrm{~d}, J=8.5 \mathrm{~Hz}, 1 \mathrm{H}, \mathrm{Ar} H), 6.05\left(\mathrm{bs}, 1 \mathrm{H}, \mathrm{O} \underline{H}\right.$ or $\left.C \underline{H}=C\left(C_{3}\right)_{2}\right), 5.99(\mathrm{~s}, 1 \mathrm{H}, \mathrm{OH}), 5.66(s, 1 \mathrm{H}, O \underline{H}$ or $\left.\mathrm{C} \underline{H}=\mathrm{C}\left(\mathrm{CH}_{3}\right)_{2}\right), 4.98\left(\mathrm{~s}, 1 \mathrm{H}, \mathrm{C}=\mathrm{CH}_{2}\right), 4.86\left(\mathrm{~s}, 1 \mathrm{H}, \mathrm{C}=\mathrm{CH}_{2}\right), 3.38\left(\mathrm{~s}, 2 \mathrm{H}, \mathrm{CH}_{2}\right), 1.97\left(\mathrm{~d}, \mathrm{~J}=1.5 \mathrm{~Hz}, 3 \mathrm{H}, \mathrm{CH}_{3}\right)$, $1.73\left(\mathrm{~s}, 3 \mathrm{H}, \mathrm{CH}_{3}\right), 1.70-1.66\left(\mathrm{~m}, 3 \mathrm{H}, \mathrm{CH}_{3}\right)^{11}$

\section{2-(2-Methylallyl)-4-nitrophenol (1e)}

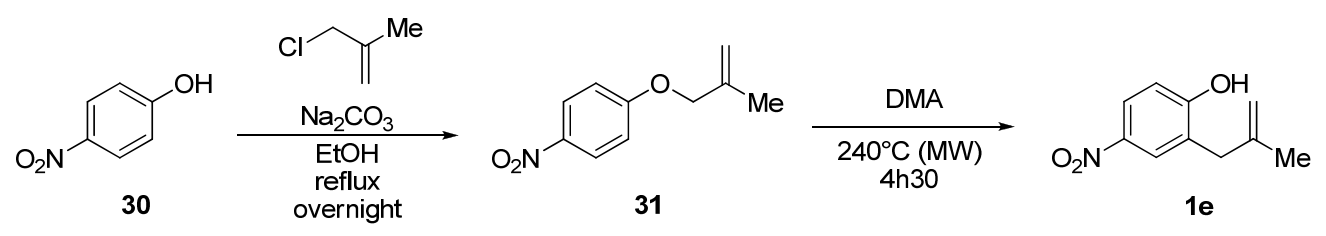

Following a slight modification of the reported procedure, ${ }^{12}$ sodium carbonate $(1.10 \mathrm{~g}, 10.3 \mathrm{mmol}, 1.30$ equiv) and methyl allyl chloride (1.0 mL, $10 \mathrm{mmol}, 1.3$ equiv) were added to a stirred solution of 4-nitro phenol (30) $(1.1 \mathrm{~g}, 7.9 \mathrm{mmol}, 1.0$ equiv) in dry ethanol $(3.0 \mathrm{~mL})$. The mixture was stirred under reflux overnight. Then it was allowed to cool to room temperature and water $(25 \mathrm{~mL})$ was added, followed by extraction with ether (3 x $10 \mathrm{~mL})$. The combined organic layers were then washed with $2 \mathrm{M} \mathrm{NaOH}(3 \mathrm{x} 20 \mathrm{~mL})$. It was dried with $\mathrm{MgSO}_{4}$, filtered and concentrated in vacuo to afford 1-(2-methylallyloxy)-4-nitrobenzene (31) (1.29 g, $\leq 6.67$ mmol, $\leq 84 \%$ yield) which was used without further purification.

1-(2-Methylallyloxy)-4-nitrobenzene (31) (500 mg, $2.58 \mathrm{mmol})$ was dissolved in DMA (2.3 $\mathrm{mL})$ and the mixture was stirred under $\mathrm{N}_{2}$ at $240{ }^{\circ} \mathrm{C}$ under microwave irradiation for $4 \mathrm{~h} 30$. DMA was evaporated in vacuo. The resulting crude product was purified by chromatography $\left(\mathrm{SiO}_{2}, \mathrm{PET} /\right.$ EtOAc 9:1-7:3) to yield 1e (179 mg, $0.926 \mathrm{mmol}, 35 \%$ yield) as a brown solid. $\mathrm{R}_{f}=0.23$, (PET/EtOAc 10:1). Mp 56-58 ${ }^{\circ} \mathrm{C} .{ }^{1} \mathrm{H} \mathrm{NMR}(400 \mathrm{MHz}$ $\left.\mathrm{CDCl}_{3}\right) \delta 8.11-8.03(\mathrm{~m}, 2 \mathrm{H}, \mathrm{Ar} H), 6.91(\mathrm{~d}, J=9.0 \mathrm{~Hz}, 1 \mathrm{H}, \mathrm{ArH}), 6.23(\mathrm{~s}, 1 \mathrm{H}, \mathrm{OH}), 5.00\left(\mathrm{~s}, 1 \mathrm{H}, \mathrm{C}=\mathrm{CH}_{2}\right)$, $4.90\left(\mathrm{~s}, 1 \mathrm{H}, \mathrm{C}=\mathrm{CH}_{2}\right), 3.44\left(\mathrm{~s}, 2 \mathrm{H}, \mathrm{CH}_{2}\right), 1.75\left(\mathrm{~s}, 3 \mathrm{H}, \mathrm{CH}_{3}\right) .{ }^{13} \mathrm{C} \mathrm{NMR}\left(100 \mathrm{MHz}, \mathrm{CDCl}_{3}\right) \delta 160.6,143.2$, 141.5, 126.9, 125.8, 124.5, 116.2, 113.8, 39.5, 22.1. IR v 3389 (broad), 2941 (m), 2865 (m), 2175 (w), 1738 (m), $1650(\mathrm{w}), 1589(\mathrm{~m}), 1523$ (m), 1497 (m), 1451 (m), $1380(\mathrm{w}), 1338(\mathrm{~s}), 1284(\mathrm{~s}), 1237(\mathrm{~m}), 1163$ (w), 1129

(11) ${ }^{1}$ H-NMR values for 1d' are reported in italics. For signals at 6.05 and 5.99 the assigned proton is underlined.

(12) Buu-Hoi, N. P. ; Jacquignon, P. ; Dufour, M. Bull. Soc. Chim. Fr. 1964, 23. 
(w), $1081(\mathrm{~m}), 1040(\mathrm{w}), 996(\mathrm{w}), 906(\mathrm{~m}), 886(\mathrm{~m}), 832(\mathrm{~m}), 758(\mathrm{~m}), 685(\mathrm{~m}) ; 676(\mathrm{~m})$. HRMS (ESI) ${ }^{[13]}$ calcd for $\mathrm{C}_{10} \mathrm{H}_{11} \mathrm{NO}_{3} \mathrm{Ag}^{+}\left(\mathrm{M}+{ }^{107} \mathrm{Ag}, \mathrm{M}+{ }^{109} \mathrm{Ag}\right)$ 299.9796, 301.9779; found 299.9731, 301.9746 .

1-(4-Hydroxy-3-(2-methylallyl)phenyl)ethanone (1f)

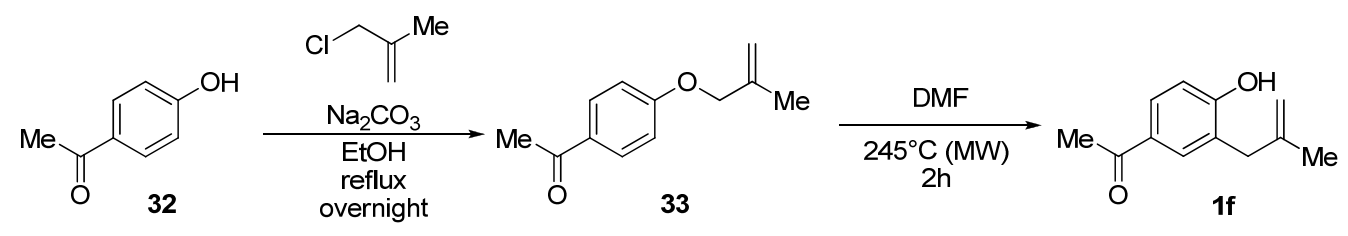

Following a slight modification of the reported procedure, ${ }^{12}$ sodium carbonate $(1.0 \mathrm{~g}, 9.7 \mathrm{mmol}, 1.2$ equiv $)$ and methyl allyl chloride (1.0 mL, $9.7 \mathrm{mmol}, 1.2$ equiv) were added to a stirred solution of 4hydroxybenzophenone (32) (1.1 g, $8.0 \mathrm{mmol}, 1.0$ equiv) in ethanol $(2.0 \mathrm{~mL})$. The mixture was refluxed overnight. Then it was allowed to cool to room temperature and water $(25 \mathrm{~mL})$ was added, followed by extraction with ether $(3 \times 10 \mathrm{~mL})$. The combined organic layers were then washed with $2 \mathrm{M} \mathrm{NaOH}(3 \times 20 \mathrm{~mL})$, dried with $\mathrm{MgSO}_{4}$, filtered and concentrated in vacuo to afford 1-(4-(2-methylallyloxy)phenyl)ethanone (33) $(1.03 \mathrm{~g}, \leq 5.40 \mathrm{mmol}, \leq 66 \%$ yield $)$ which was used without further purification.

1-(4-(2-Methylallyloxy)phenyl)ethanone (33) (500 mg, $2.63 \mathrm{mmol})$ was dissolved in DMF (2.3 $\mathrm{mL})$ and the mixture was stirred under $\mathrm{N}_{2}$ at $245^{\circ} \mathrm{C}$ under microwave irradiation for $2 \mathrm{~h}$. DMF was evaporated in vacuo. The resulting crude product was purified by chromatography $\left(\mathrm{SiO}_{2}, \mathrm{PET} /\right.$ EtOAc 9:1-7:3) to yield $1 \mathbf{1 f}(324 \mathrm{mg}, 1.70$ mmol, 64\% yield) as a pale yellow oil. $\mathrm{R}_{f}=0.28$, (PET/EtOAc 5:1). ${ }^{1} \mathrm{H}$ NMR $\left(400 \mathrm{MHz}, \mathrm{CDCl}_{3}\right) \delta 7.81-7.76$ $(\mathrm{m}, 2 \mathrm{H}, \operatorname{Ar} H), 6.87(\mathrm{~d}, J=8.2 \mathrm{~Hz}, 1 \mathrm{H}, \mathrm{ArH}), 5.75(\mathrm{~s}, 1 \mathrm{H}, \mathrm{OH}), 4.97\left(\mathrm{~s}, 1 \mathrm{H}, \mathrm{C}=\mathrm{CH}_{2}\right), 4.89\left(\mathrm{~s}, 1 \mathrm{H}, \mathrm{C}=\mathrm{CH}_{2}\right)$, $3.43\left(\mathrm{~s}, 2 \mathrm{H}, \mathrm{CH}_{2}\right), 2.55\left(\mathrm{~s}, 3 \mathrm{H}, \mathrm{COCH}_{3}\right), 1.75\left(\mathrm{~s}, 3 \mathrm{H}, \mathrm{C}=\mathrm{CCH}_{3}\right) .{ }^{13} \mathrm{C} \mathrm{NMR}\left(100 \mathrm{MHz}, \mathrm{CDCl}_{3}\right) \delta 198.5,160.0$, 144.1, 132.0, 129.7, 129.4, 125.7, 115.7, 112.5, 38.9, 26.3, 22.3. IR v 3225 (broad, w), 2942 (m), 2864 (m), $2361(\mathrm{w}), 2176$ (m), 1676 (s), 1590 (s), 1523 (m), 1489 (m), 1463 (m), 1437 (m), 1380 (w), 1357 (s), 1326 (w), 1266 (s), 1245 (s), $1171(\mathrm{w}), 1112(\mathrm{w}), 1075$ (m), 1057 (m), 1034 (m), 996 (w), 958 (w), 911 (m), 883 (s), 817 (m), $787(\mathrm{w}), 729(\mathrm{w}), 676(\mathrm{~s})$. HRMS (ESI) calcd for $\mathrm{C}_{12} \mathrm{H}_{15} \mathrm{O}_{2}(\mathrm{M}+\mathrm{H})$ 191.1072; found 191.1075.

\section{5-Bromo-2-(2-methylallyl)phenol (1g)}

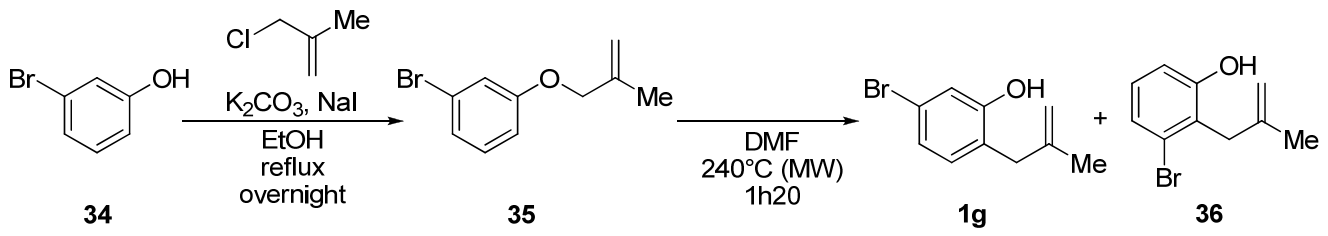

Following a slight modification of the reported procedure, ${ }^{10}$ potassium carbonate $(1.45 \mathrm{~g}, 10.5 \mathrm{mmol}, 1.3$ equiv), methyl allyl chloride (1.2 mL, $12 \mathrm{mmol}, 1.5$ equiv) and $\mathrm{NaI}$ (tip of a spatula) were added to a stirred solution of 3-bromo phenol (34) (1.4 g, $8.1 \mathrm{mmol}, 1.0$ equiv) in dry ethanol (4.5 mL). The mixture was stirred

(13) ESI mass spectrum of compound 1e was acquired after treatment of 1e with $\mathrm{AgNO}_{3}$, as a consequence of troublesome ionization of the pure compound. 
under reflux overnight. Then it was allowed to cool to room temperature and water $(25 \mathrm{~mL})$ was added, followed by extraction with ether $(3 \times 10 \mathrm{~mL})$. The combined organic layers were then washed with $2 \mathrm{M} \mathrm{NaOH}$ (3 $\times 20 \mathrm{~mL}$ ), dried with $\mathrm{MgSO}_{4}$, filtered and concentrated in vacuo to afford 1-bromo-3-(2methylallyloxy)benzene (35) (1.3 g, $\leq 7.3 \mathrm{mmol}, \leq 90 \%$ yield) which was used without further purification.

1-Bromo-3-(2-methylallyloxy)benzene (35) (0.68 g, $3.0 \mathrm{mmol})$ was dissolved in DMF (2.4 mL) and the mixture was stirred under $\mathrm{N}_{2}$ at $240{ }^{\circ} \mathrm{C}$ under microwave irradiation for $1 \mathrm{~h} 20$. DMF was evaporated in vacuo. The resulting crude product was purified by chromatography $\left(\mathrm{SiO}_{2}, \mathrm{PET} / \mathrm{EtOAc}\right.$ 97:3) to yield two products: 36 $\left(\mathrm{R}_{f}=0.62\right.$, PET/EtOAc 10:1; not separable from a non identified by-product), and 5-bromo-2-(2methylallyl)phenol $1 \mathrm{~g}\left(\mathrm{R}_{f}=0.51\right.$, PET/EtOAc 10:1; pure, $0.12 \mathrm{~g}, 0.53 \mathrm{mmol}, 18 \%$ yield). Characterization data for 1g: $\mathrm{R}_{f}=0.62$, $(\mathrm{PET} / \mathrm{EtOAc} 10: 1) .{ }^{1} \mathrm{H}$ NMR $\left(400 \mathrm{MHz}, \mathrm{CDCl}_{3}\right) \delta 7.17(\mathrm{dd}, J=8.2,1.2 \mathrm{~Hz}, 1 \mathrm{H}, \mathrm{ArH}), 7.00$ $(\mathrm{t}, J=8.2 \mathrm{~Hz}, 1 \mathrm{H}, \operatorname{Ar} H), 6.79(\mathrm{~d}, J=8.1 \mathrm{~Hz}, 1 \mathrm{H}, \mathrm{Ar} H), 5.30(\mathrm{~s}, 1 \mathrm{H}, \mathrm{OH}), 4.91\left(\mathrm{~m}, 1 \mathrm{H}, \mathrm{C}=C_{2}\right), 4.74(\mathrm{~m}, 1$ $\left.\mathrm{H}, \mathrm{C}=\mathrm{CH}_{2}\right), 3.62\left(\mathrm{~s}, 2 \mathrm{H}, \mathrm{CH}_{2}\right), 1.80\left(\mathrm{~s}, 3 \mathrm{H}, \mathrm{CH}_{3}\right) .{ }^{13} \mathrm{C} \mathrm{NMR}\left(100 \mathrm{MHz}, \mathrm{CDCl}_{3}\right) \delta 155.6,143.4,128.6,125.9$, 125.3, 115.3, 115.3, 112.0, 38.8, 22.3. IR v 3430 (broad), 3080 (w), 2973 (w), 2914 (w), 2847 (w), 1743 (m), $1650(\mathrm{~m}), 1580(\mathrm{~s}), 1448(\mathrm{~s}), 1374(\mathrm{w}), 1320(\mathrm{~m}), 1276$ (s), $1226(\mathrm{~m}), 1186(\mathrm{~m}), 1120(\mathrm{w}), 1019(\mathrm{w}), 928(\mathrm{~s})$, $893(\mathrm{~m}), 856(\mathrm{~s}), 818(\mathrm{w}), 772(\mathrm{~s}), 721(\mathrm{~m}), 676(\mathrm{w}), 648(\mathrm{w})$. HRMS (ESI) ${ }^{14}$ expected for $\mathrm{C}_{10} \mathrm{H}_{11}{ }^{79} \mathrm{BrO}^{107} \mathrm{Ag}^{+}$ $\left(\mathrm{M}+{ }^{107} \mathrm{Ag}\right) 332.9035$; found 332.8206 .

\section{2-Bromo-6-(2-methylallyl)phenol (1h)}

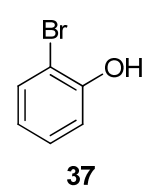

37
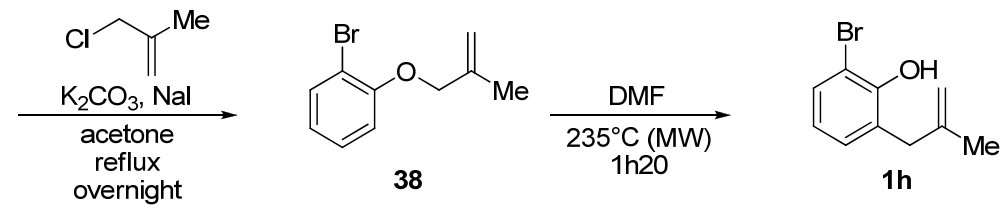

Following a slight modification of the reported procedure, ${ }^{10}$ potassium carbonate $(1.45 \mathrm{~g}, 10.5 \mathrm{mmol}, 1.3$ equiv), methyl allyl chloride (1.2 mL, $12 \mathrm{mmol}, 1.5$ equiv) and $\mathrm{NaI}$ (tip of a spatula) were added to a stirred solution of 2-bromophenol (37) (1.4 g, $8.1 \mathrm{mmol}, 1.0$ equiv) in dry acetone $(4.5 \mathrm{~mL})$. The mixture was stirred under reflux overnight. Then it was allowed to cool to room temperature and water (25 $\mathrm{mL})$ was added, followed by extraction with ether $(3 \times 10 \mathrm{~mL})$. The combined organic layers were then washed with $2 \mathrm{M} \mathrm{NaOH}$ (3 $\mathrm{x} 20 \mathrm{~mL}$ ), dried with $\mathrm{MgSO}_{4}$, filtered and concentrated in vacuo to afford 1-bromo-2-(2methylallyloxy)benzene (38) (1.73 g, $\leq 7.69 \mathrm{mmol}, \leq 95 \%$ yield) which was used without further purification.

1-Bromo-2-(2-methylallyloxy)benzene (38) (650 mg, $2.87 \mathrm{mmol})$ was dissolved in DMF (2.3 $\mathrm{mL})$ and the mixture was stirred under $\mathrm{N}_{2}$ at $235^{\circ} \mathrm{C}$ under microwave irradiation for $1 \mathrm{~h} 20$. DMF was evaporated in vacuo. The resulting crude product was purified by chromatography $\left(\mathrm{SiO}_{2}\right.$, PET/EtOAc 30:1-20:1) to yield $\mathbf{1 h}$ (412 mg, 1.82 mmol, 61\% yield) as a yellow oil. $\mathrm{R}_{\mathrm{f}} 0.84$ (PET/EtOAc 10:1). ${ }^{1} \mathrm{H}$ NMR (400 MHz, $\left.\mathrm{CDCl}_{3}\right) \delta 7.34(\mathrm{dd}$,

(14) ESI mass spectrum of compound $\mathbf{1 g}$ was acquired after treatment of $\mathbf{1 g}$ with $\mathrm{AgNO}_{3}$, as a consequence of troublesome ionization of the pure compound. 
$J=7.9,1.4 \mathrm{~Hz}, 1 \mathrm{H}, \operatorname{Ar} H), 7.07(\mathrm{~d}, J=7.4 \mathrm{~Hz}, 1 \mathrm{H}, \operatorname{Ar} H), 6.76(\mathrm{t}, J=7.7 \mathrm{~Hz}, 1 \mathrm{H}, \operatorname{Ar} H), 5.61(\mathrm{~s}, 1 \mathrm{H}, \mathrm{OH})$, $4.85\left(\mathrm{~s}, 1 \mathrm{H}, \mathrm{C}=\mathrm{CH}_{2}\right), 4.71\left(\mathrm{~s}, 1 \mathrm{H}, \mathrm{C}=\mathrm{CH}_{2}\right), 3.39\left(\mathrm{~s}, 2 \mathrm{H}, \mathrm{CH}_{2}\right), 1.75\left(\mathrm{~s}, 3 \mathrm{H}, \mathrm{CH}_{3}\right) .{ }^{13} \mathrm{C} \mathrm{NMR}(100 \mathrm{MHz}$, $\left.\mathrm{CDCl}_{3}\right) \delta 150.6,144.1,131.1,130.3,130.2,121.4,112.1,110.6,38.9,22.4$; IR v $3511(\mathrm{~m}), 3075(\mathrm{w}), 3028(\mathrm{w})$, 2973 (w), 1915 (w), 1885 (w), 1797 (w), 1650 (m), 1599 (m), 1449 (s), 1375 (m), 1328 (s), 1238 (s), 1210 (m), $1166(\mathrm{~m}), 1115(\mathrm{~m}), 1014$ (w), 893 (s), 863 (s), 820 (w), 764 (s), 732 (s), 636 (m). HRMS (ESI) ${ }^{15}$ calcd for $\mathrm{C}_{10} \mathrm{H}_{11}{ }^{79} \mathrm{BrO}^{109} \mathrm{Ag}^{+}\left(\mathrm{M}+{ }^{109} \mathrm{Ag}\right), \mathrm{C}_{10} \mathrm{H}_{11}{ }^{81} \mathrm{BrO}^{109} \mathrm{Ag}^{+}\left(\mathrm{M}+{ }^{109} \mathrm{Ag}\right)$ 334.9032, 336.9022; found 334.9037, 336.9014.

\section{2-(3-Methylbut-3-enyl)phenol (1j)}

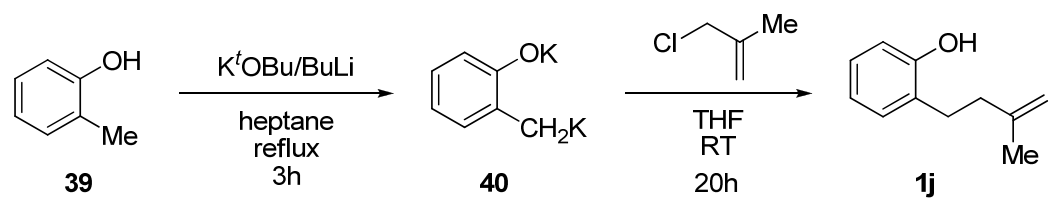

Following a reported procedure, ${ }^{16}$ Ar was bubbled through heptane $(130 \mathrm{~mL})$ for $15 \mathrm{~min}$, and ${ }^{t} \mathrm{BuOK}(5.0 \mathrm{~g}$, $45 \mathrm{mmol}, 4.0$ equiv) and ${ }^{n} \mathrm{BuLi}(2.5 \mathrm{M}$ in hexanes, $18 \mathrm{~mL}, 45 \mathrm{mmol}, 4.0$ equiv) were added. The resulting orange mixture was stirred under Ar at room temperature for $15 \mathrm{~min}$ and then $o$-cresol (39) (1.2 g, $11 \mathrm{mmol}, 1.0$ equiv) was added, whereas the reaction mixture turned to bright yellow; refluxing $3 \mathrm{~h}$ turned it brown. The mixture was then cooled to $0^{\circ} \mathrm{C}$ and the dianion salt $\mathbf{4 0}$ was filtered via cannula and washed with dry hexane $(100 \mathrm{~mL})$. The salt was dissolved in THF $\left(100 \mathrm{~mL}\right.$, previously cooled to $\left.0^{\circ} \mathrm{C}\right)$ and the resulting suspension was transferred via cannula into a solution of methallyl chloride $(3.3 \mathrm{~mL}, 33 \mathrm{mmol}, 3.0$ equiv) in THF (100 mL). The mixture was then stirred at room temperature for $20 \mathrm{~h}$. Water was then added dropwise $(10 \mathrm{~mL})$ and the solution was acidified with $5 \mathrm{M} \mathrm{HCl}$. The aqueous layer was separated and extracted with chloroform $(3 \mathrm{x} 50$ $\mathrm{mL}$ ). The combined organic layers were washed with brine, dried over $\mathrm{MgSO}_{4}$, filtered and evaporated under reduced pressure. The crude product was purified by column chromatography $\left(\mathrm{SiO}_{2}, \mathrm{PET} / \mathrm{EtOAc} 99: 1-15: 1\right)$ to afford 1j (0.70 g, $3.9 \mathrm{mmol}, 35 \%$ yield) as a yellow oil. Rf 0.64, (PET/EtOAc 5:1). $\left.{ }^{1} \mathrm{H} \mathrm{NMR} \mathrm{(400} \mathrm{MHz} \mathrm{CDCl}_{3}\right)$ $\delta 7.13(\mathrm{dd}, J=7.4,1.5 \mathrm{~Hz}, 1 \mathrm{H}, \mathrm{Ar} H), 7.09(\mathrm{td}, J=7.6,1.5 \mathrm{~Hz}, 1 \mathrm{H}, \mathrm{ArH}), 6.88(\mathrm{td}, J=7.2,1.2 \mathrm{~Hz}, 1 \mathrm{H}, \mathrm{ArH})$, $6.76(\mathrm{td}, J=7.9,1.0 \mathrm{~Hz}, 1 \mathrm{H}, \mathrm{ArH}), 4.76\left(\mathrm{~m}, 2 \mathrm{H}, \mathrm{C}=\mathrm{CH}_{2}\right), 4.69(\mathrm{bs}, 1 \mathrm{H}, \mathrm{OH}), 2.76\left(\mathrm{~m}, 2 \mathrm{H}, \mathrm{CH}_{2}\right), 2.32(\mathrm{~m}, 2$ $\left.\mathrm{H}, \mathrm{CH}_{2}\right), 1.79\left(\mathrm{~s}, 3 \mathrm{H}, \mathrm{CH}_{3}\right) .{ }^{13} \mathrm{C} \mathrm{NMR}\left(100 \mathrm{MHz}, \mathrm{CDCl}_{3}\right) \delta 153.6,145.8,130.0,128.3,127.0,120.5,115.2$, 110.1, 37.7, 28.5, 22.6. IR v 3406 (broad, m), 3073 (m), 3035 (m), 2968 (m), 2932 (m), 2857 (m), 1785 (w), 1648 (m), 1609 (m), 1592 (m), 1500 (m), 1455 (s), 1374 (m), 1332 (m), 1296 (sh), 1236 (s), 1173 , (m) 1099 (m), $1045(\mathrm{w}), 932(\mathrm{w}), 889(\mathrm{~m}), 838(\mathrm{w}), 752(\mathrm{~s}), 708,674(\mathrm{w}), 673(\mathrm{w}), 657(\mathrm{w}), 647(\mathrm{w}), 633(\mathrm{w}), 632(\mathrm{w})$, 609 (w). Characterization data corresponded to the literature values. ${ }^{16}$

\section{2-(Prop-2-en-1-yl)benzoic acid (5a)}

(15) ESI mass spectrum of compound $\mathbf{1 h}$ was acquired after treatment of $\mathbf{1 h}$ with $\mathrm{AgNO}_{3}$, as a consequence of troublesome ionization of the pure compound.

(16) Bates, R. B.; Siahaan, T. J. J. Org. Chem. 1986, 51, 1432. 


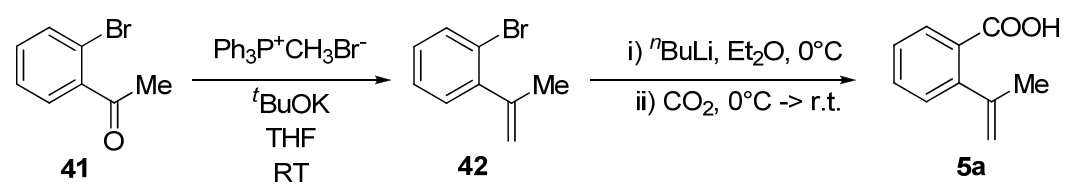

Following a reported procedure, ${ }^{17}$ a solution of potassium tert-butoxide $(2.0 \mathrm{~g}, 18 \mathrm{mmol}, 1.2$ equiv) in THF $(18 \mathrm{~mL})$ was added to a stirred suspension of metylphenylphosphonium bromide ( $6.4 \mathrm{~g}, 18 \mathrm{mmol}, 1.2$ equiv) in THF ( $45 \mathrm{~mL}$ ) under $\mathrm{N}_{2}$ at room temperature. The mixture was stirred for 15 minutes and then a solution of 2'bromoacetophenone (41) $(2.0 \mathrm{~mL}, 15 \mathrm{mmol}, 1.0$ equiv) in THF $(30 \mathrm{~mL})$ was added via cannula. The resulting reaction mixture was stirred for $3 \mathrm{~h}$ at room temperature and quenched by the addition of a saturated solution of ammonium chloride $(60 \mathrm{~mL})$. The aqueous layer was extracted with ether $(3 \times 50 \mathrm{~mL})$ and the combined organic layers were washed with water and brine, filtered and concentrated in vacuo. The resulting colorless solid was triturated with hexane $(100 \mathrm{~mL})$ to separate $\mathrm{Ph}_{3} \mathrm{P}=\mathrm{O}$ which was removed by filtration. The filtrate was concentrated under reduced pressure. Purification by column chromatography $\left(\mathrm{SiO}_{2}\right.$, hexane) afforded bromostyrene 42 ( $2.45 \mathrm{~g}, 12.5 \mathrm{mmol}, 83 \%$ yield) as a colorless oil.

A solution of bromostyrene $42\left(600 \mathrm{mg}, 3.06 \mathrm{mmol}, 1.00\right.$ equiv) in $\mathrm{Et}_{2} \mathrm{O}(6.1 \mathrm{~mL}$, anhydrous, previously flushed with Ar) was treated dropwise with ${ }^{n} \mathrm{BuLi}\left(2.5 \mathrm{~m}\right.$ in hexane, $1.5 \mathrm{~mL}, 3.7 \mathrm{mmol}, 1.2$ equiv) at $0{ }^{\circ} \mathrm{C}$. The reaction mixture was stirred at $0{ }^{\circ} \mathrm{C}$ for 10 minutes and then anhydrous $\mathrm{CO}_{2}$ was bubbled through it for 10 minutes. The mixture was allowed to warm to room temperature and stirred for an additional 30 minutes. The reaction was quenched with $\mathrm{NaHCO}_{3}$ (saturated solution, $30 \mathrm{~mL}$ ). The aqueous layer was washed with diethyl ether $(2 \times 30 \mathrm{~mL})$ and then acidified with $2 \mathrm{~N} \mathrm{HCl}$ to $\mathrm{pH} 1$ and extracted with ether $(3 \times 30 \mathrm{~mL})$. The combined organic layers were dried over $\mathrm{MgSO}_{4}$, filtered and concentrated under reduced pressure to afford benzoic acid 5a (414 mg, $2.55 \mathrm{mmol}, 83 \%$ yield) as a colorless solid. $\mathrm{R}_{\mathrm{f}} 0.33$ (PET/EtOAc 5:1). Mp 67-69 ${ }^{\circ} \mathrm{C} ; 63-66^{\circ} \mathrm{C} .{ }^{17}{ }^{1} \mathrm{H}$ NMR $\left(400 \mathrm{MHz}, \mathrm{CDCl}_{3}\right) \delta 11.1$ (bs, $\left.1 \mathrm{H}, \mathrm{COO} H\right), 7.98(\mathrm{dd}, J=7.9,1.4 \mathrm{~Hz}, 1 \mathrm{H}, \mathrm{Ar} H), 7.53(\mathrm{td}, J=7.6,1.4 \mathrm{~Hz}$, $1 \mathrm{H}, \operatorname{Ar} H), 7.38(\mathrm{td}, J=7.7,1.4 \mathrm{~Hz}, 1 \mathrm{H}, \operatorname{Ar} H), 7.34-7.18(\mathrm{~m}, 1 \mathrm{H}, \mathrm{Ar} H), 5.17$ (q, $J=1.5 \mathrm{~Hz}, 1 \mathrm{H}, \mathrm{C}=C_{2}$ ), 4.96 (m, $\left.1 \mathrm{H}, \mathrm{C}=\mathrm{CH}_{2}\right), 2.15\left(\mathrm{~s}, 3 \mathrm{H}, \mathrm{CH}_{3}\right) .{ }^{13} \mathrm{C} \mathrm{NMR}\left(100 \mathrm{MHz}, \mathrm{CDCl}_{3}\right) \delta 173.5,146.6,146.3,132.6,130.8,129.8$, 128.0, 127.1, 114.0, 24.3. IR v 3078 (m), 3077 (m), 3010 (m), 2978 (m), 2916 (m), 2863 (m), 2823 (m), 2652 (w), 1693 (s), 1641 (w), 1598 (w), 1570 (w), 1487(w), 1452 (w), 1408 (m), 1372 (w), 1299 (s), 1266 (s), 1141 (w), $1076(\mathrm{w}), 897(\mathrm{~s}), 804(\mathrm{w}), 768(\mathrm{~s}), 737(\mathrm{~m}), 718(\mathrm{~m}), 651(\mathrm{~m}) .{ }^{1} \mathrm{H}$ NMR spectra corresponded to the literature values. ${ }^{18}$

\section{4-methoxy-2-(prop-1-en-2-yl)benzoic acid (5b)}

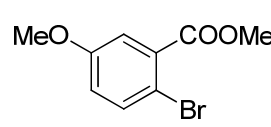

43

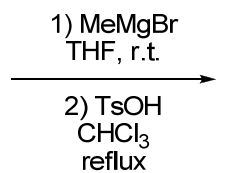

reflux

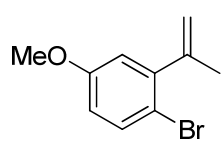

44

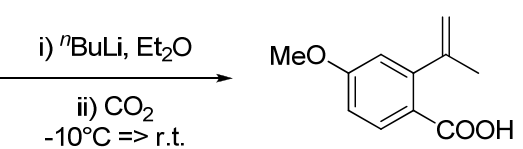

$5 \mathbf{b}$

(17) Trend, R. M.; Ramtohul, Y. K.; Stoltz, B. M. J. Am. Chem. Soc. 2005, 127, 17778.

(18) Hellwinkel, D.; Aulmich, G.; Melan, M. Chem. Ber. 1981, 114, 86. 
Following a slight modification of a reported procedure, ${ }^{19}$. methyl 2-bromo-5-methoxybenzoate (43) (0.73 g, $3.0 \mathrm{mmol}, 1.0$ equiv) was added dropwise to a solution of $\operatorname{MeMgBr}\left(3 \mathrm{M}\right.$ in $\left.\mathrm{Et}_{2} \mathrm{O}\right)(2.1 \mathrm{~mL}, 6.2 \mathrm{mmol}, 2.1$ equiv) in THF $(6 \mathrm{~mL})$. The resulting colorless suspension was stirred under nitrogen for $20 \mathrm{~h}$ and then the reaction was quenched by adding a saturated solution of $\mathrm{NH}_{4} \mathrm{Cl}(7 \mathrm{~mL})$. The aqueous layer was exctracted with $\mathrm{Et}_{2} \mathrm{O}(3 \times 10$ $\mathrm{mL}$ ) and the combined organic layers were washed with brine, dried over $\mathrm{MgSO}_{4}$, filtered and concentrated in vacuo to afford the expected tertiary alcohol $(680 \mathrm{mg})$ as a colorless solid which was used without further purification. The alcohol was dissolved in chloroform $(7.3 \mathrm{~mL})$ and toluenesulfonic acid $(7 \mathrm{mg}, 0.04 \mathrm{mmol}$, 0.01 equiv) and the mixture was refluxed for $1 \mathrm{~h}$. The reaction was then quenched by addition of a saturated solution of $\mathrm{NaHCO}_{3}(10 \mathrm{~mL})$ followed by extraction of the aqueous layer with $\mathrm{Et}_{2} \mathrm{O}(3 \times 10 \mathrm{~mL})$. The combined organic layers were dried over $\mathrm{MgSO}_{4}$, filtered and the solvent was evaporated under reduced pressure. The crude procduct was purified by column chromatography $\left(\mathrm{SiO}_{2}, \mathrm{PET} / \mathrm{EtOAc} 98: 2\right)$ to afford the bromide $\mathbf{4 4}$ (513 mg, $2.26 \mathrm{mmol}, 76 \%$ yield) as a colorless solid.

Following a reported procedure, ${ }^{17}$ bromide 44 (0.25 g, $1.1 \mathrm{mmol}, 1.0$ equiv) was dissolved in Ar-flushed $\mathrm{Et}_{2} \mathrm{O}(2.2 \mathrm{~mL})$ and the solution was cooled to $-10^{\circ} \mathrm{C}$. After 5 minutes ${ }^{n} \mathrm{BuLi}(2.5 \mathrm{M}$ in hexanes $)(0.53 \mathrm{~mL}, 1.3$ mmol, 1.2 equiv) was added dropwise and the resulting orange mixture was stirred at $-10^{\circ} \mathrm{C}$ for 20 min. Then $\mathrm{CO}_{2}$ was bubbled through the mixture for $15 \mathrm{~min}$ at $-10^{\circ} \mathrm{C}$ and the solution was then allowed to warm to room temperature and stirred for $40 \mathrm{~min}$. The reaction was quenched by adding a saturated solution of $\mathrm{NaHCO}_{3}(5$ $\mathrm{mL})$. The organic layer was separated and washed with a 1 M solution of $\mathrm{NaOH}(3 \times 10 \mathrm{~mL})$. The combined basic layers were acidified with a $1 \mathrm{M}$ solution of $\mathrm{HCl}$ until $\mathrm{pH} 1$ and then extracted with $\mathrm{Et}_{2} \mathrm{O}(3 \mathrm{x} 10 \mathrm{~mL})$. The combined organic layers were dried over $\mathrm{MgSO}_{4}$, filtered and the solvent was evaporated under reduced pressure. The crude yellow solid was purified by recrystallization from Hexane/EtOAc to furnish 4-methoxy-2(prop-1-en-2-yl)benzoic acid (5b) $(97 \mathrm{mg}, 0.50 \mathrm{mmol}, 46 \%)$ as a colorless solid. $\mathrm{R}_{\mathrm{f}} 0.23$ (PET/EtOAc 5: 1). Mp $109-111^{\circ} \mathrm{C} .{ }^{1} \mathrm{H}$ NMR $\left(400 \mathrm{MHz}, \mathrm{CDCl}_{3}\right) \delta 12.00$ (br s, $\left.1 \mathrm{H}, \mathrm{COOH}\right), 8.01(\mathrm{~d}, J=8.7 \mathrm{~Hz}, 1 \mathrm{H}, \mathrm{Ar} H), 6.85(\mathrm{dd}$, $\left.J_{1}=8.7,2.6 \mathrm{~Hz}, 1 \mathrm{H}, \mathrm{ArH}\right), 6.74(\mathrm{~d}, J=2.7 \mathrm{~Hz}, 1 \mathrm{H}, \mathrm{ArH}), 5.11\left(\mathrm{~m}, 1 \mathrm{H}, \mathrm{C}=\mathrm{CH}_{2}\right), 4.88\left(\mathrm{~m}, 1 \mathrm{H}, \mathrm{C}=\mathrm{CH}_{2}\right), 3.87$ $\left(\mathrm{s}, 3 \mathrm{H}, \mathrm{OCH}_{3}\right), 2.11\left(\mathrm{~s}, 3 \mathrm{H}, \mathrm{CH}_{3}\right) .{ }^{13} \mathrm{C} \mathrm{NMR}\left(100 \mathrm{MHz}, \mathrm{CDCl}_{3}\right) \delta 172.2,162.9,149.2,147.2,133.5,119.8$, 115.3, 113.2, 112.2, 55.4, 24.2. IR v 3380 (br, m), 2946 (s), 2893 (m), 2866 (s), 2177 (w), 2176 (s), 1780 (m), 1705 (w), 1541 (w), 1514 (w), 1462 (m), 1425 (w), 1389 (w), 1298 (w), 1274 (w), 1233 (w), 1179 (m), 1024 (m), 921 (w), $884(\mathrm{~m}), 839$ (w), 791 (w), 742 (w), 680 (m), 659 (m), 633 (m). HRMS (ESI) calcd for $\mathrm{C}_{11} \mathrm{H}_{13} \mathrm{O}_{3}{ }^{+}$ $(\mathrm{M}+\mathrm{H})$ 191.0708; found 191.0704.

\section{2-(1-Phenylvinyl)benzoic acid (5c)}




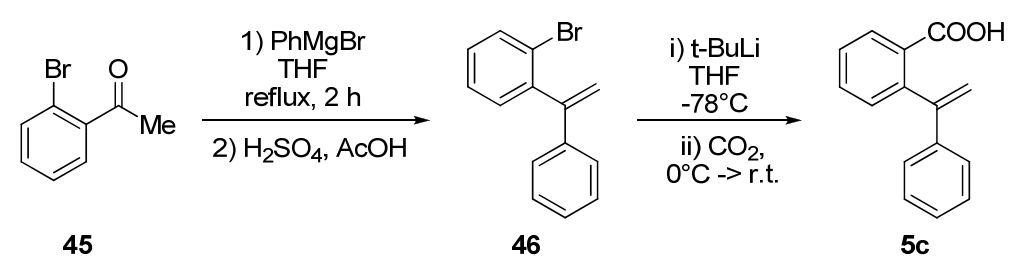

Following a reported procedure, ${ }^{20}$ a solution of 2'-bromacetophenone (45) $(2.0 \mathrm{~mL}, 15 \mathrm{mmol}, 1.0$ equiv) in THF ( $2 \mathrm{~mL})$ was added dropwise to a stirred solution of phenyl magnesium bromide in THF $(1 \mathrm{M}, 16.6 \mathrm{~mL}$, $16.6 \mathrm{mmol}, 1.10$ equiv). The resulting mixture was heated at reflux for $2 \mathrm{~h}$. It was then allowed to cool to room temperature and the reaction was quenched by addition of saturated $\mathrm{NH}_{4} \mathrm{Cl}(15 \mathrm{~mL})$. The aqueous layer was extracted with diethyl ether $(3 \times 20 \mathrm{~mL})$ and the combined organic layers were washed with water, dried over $\mathrm{MgSO}_{4}$, filtered and concentrated in vacuo to afford the crude carbinol (2.3 g, $\left.8.5 \mathrm{mmol}\right)$. The crude carbinol was treated with a solution of $\mathrm{H}_{2} \mathrm{SO}_{4}$ in acetic acid $(4 \mathrm{~mL}, 20 \% \mathrm{v} / \mathrm{v})$ at $50{ }^{\circ} \mathrm{C}$ for 5 minutes. The mixture was then poured into a $\mathrm{Et}_{2} \mathrm{O} /$ water two-phase system $(1: 1,100 \mathrm{~mL})$. The aqueous layer was extracted with diethyl ether $(2 \times 100 \mathrm{~mL})$ and the combined organic layers were washed with an aqueous solution of $\mathrm{NaHCO}_{3}(1 \mathrm{M}, 25$ $\mathrm{mL}$ ), dried over $\mathrm{MgSO}_{4}$, filtered and concentrated under reduced pressure. The crude product was purified by column chromatography $\left(\mathrm{SiO}_{2}, \mathrm{PET}\right)$ to furnish 1-bromo-2-(1-phenylvinyl)benzene (46) (2.2 g, $8.5 \mathrm{mmol}, 56 \%$ yield) as a colorless oil.

2-(1-Phenylvinyl)benzene (46) (0.60 g, $2.3 \mathrm{mmol}, 1.0$ equiv) was dissolved in $\mathrm{Et}_{2} \mathrm{O}$ (4.5 mL, anhydrous, previously flushed with argon) and the resulting colorless mixture was cooled to $-78{ }^{\circ} \mathrm{C}$ and stirred for 5 minutes. ${ }^{t} \mathrm{BuLi}$ (1.6 $\mathrm{M}$ in pentane, $3.4 \mathrm{~mL}, 5.5 \mathrm{mmol}, 2.3$ equiv) was added dropwise, whereas the reaction mixture turned to yellow. After 50 minutes, $\mathrm{CO}_{2}$ was bubbled through the mixture for 10 minutes. The mixture turned to black, then to red and finally to yellow. It was then warmed to room temperature and stirred for an additional 30 minutes. The reaction was quenched by adding $\mathrm{NaHCO}_{3}$ (saturated aqueous solution, $20 \mathrm{~mL}$ ). The aqueous layer was washed with diethyl ether $(3 \times 20 \mathrm{~mL})$, acidified to $\mathrm{pH} 1$ with $2 \mathrm{~N} \mathrm{HCl}$ and extracted with diethyl ether $(3 \times 20 \mathrm{~mL})$. The combined organic layers were dried over $\mathrm{MgSO}_{4}$, filtered and concentrated in vacuo, to afford the crude product as a yellow foam. Purification by crystallization from hexane furnished 2-(1phenylvinyl)benzoic acid (5c) (90 mg, $0.40 \mathrm{mmol}, 17 \%$ yield) as a colorless solid. $\mathrm{R}_{\mathrm{f}}$ 0.10, (PET/EtOAc 5:1). ${ }^{1} \mathrm{H}$ NMR (400 MHz, $\mathrm{CDCl}_{3}$ ) $\delta 10.8$ (bs, 1H, COOH), 7.92 (dd, J=7.7, $\left.1.0 \mathrm{~Hz}, 1 \mathrm{H}, \mathrm{ArH}\right), 7.56(\mathrm{td}, J=7.6,1.4$ $\mathrm{Hz}, 1 \mathrm{H}, \mathrm{ArH}), 7.43(\mathrm{td}, J=7.7,1.2 \mathrm{~Hz}, 1 \mathrm{H}, \mathrm{Ar} H), 7.36(\mathrm{dd}, J=7.6,0.7 \mathrm{~Hz}, 1 \mathrm{H}, \mathrm{ArH}), 7.29-7.18(\mathrm{~m}, 5 \mathrm{H}$, $\mathrm{PhH}), 5.67\left(\mathrm{~d}, J=0.7 \mathrm{~Hz}, 1 \mathrm{H}, \mathrm{C}=\mathrm{CH}_{2}\right), 5.22\left(\mathrm{~d}, J=0.7 \mathrm{~Hz}, 1 \mathrm{H}, \mathrm{C}=\mathrm{CH}_{2}\right)$. Due to the low stability of the product, no further characterization was possible for $\mathbf{5 c}$.

\section{2-(But-2-en-2-yl)benzoic acid (5d)}




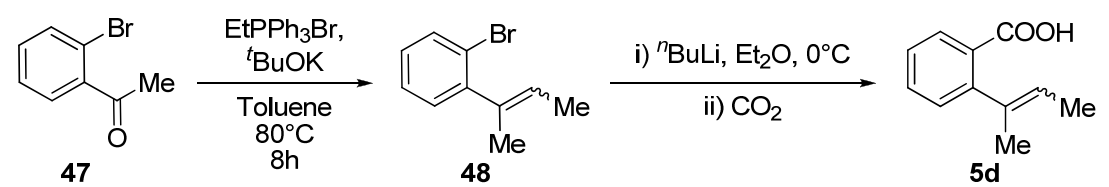

Following a reported procedure, ${ }^{17}$ ethyltriphenylphosphonium bromide (4.4 g, 12 mmol, 2.7 equiv) was added to a suspension of potassium $t$-butoxide $(1.35 \mathrm{~g}, 12.0 \mathrm{mmol}, 2.70$ equiv) in toluene $(44 \mathrm{~mL})$. The resulting orange suspension was stirred at $0{ }^{\circ} \mathrm{C}$ for 10 minutes, allowed to warm to room temperature and stirred for an additional $1 \mathrm{~h}$. The mixture was cooled to $0{ }^{\circ} \mathrm{C}$ and 2'-bromoacetophenone (47) (0.88 g, 4.4 mmol, 1.0 equiv) was added dropwise. The mixture was heated at reflux for $8 \mathrm{~h}$, then cooled to room temperature and quenched with $\mathrm{NH}_{4} \mathrm{Cl}$ (saturated aqueous solution, $50 \mathrm{~mL}$ ). The aqueous layer was extracted with diethyl ether $\left(3 \times 50 \mathrm{~mL}\right.$ ) and the combined organic layers were dried over $\mathrm{MgSO}_{4}$, filtered and concentrated under reduced pressure. The resulting colorless solid was triturated with hexane $(50 \mathrm{~mL})$ and $\mathrm{Ph}_{3} \mathrm{P}=\mathrm{O}$ was removed by filtration. The filtrate was concentrated in vacuo and purified by column chromatography ( $\mathrm{SiO}_{2}$, hexane) to afford bromostyrene 48 (679 mg, $3.23 \mathrm{mmol}, 73 \%$ yield, mixture of $\mathrm{E}$ and $\mathrm{Z}$ isomers) as a colorless oil.

${ }^{n} \operatorname{BuLi}(2.5 \mathrm{~m}$ in hexanes, $0.80 \mathrm{~mL}, 2.1 \mathrm{mmol}, 1.2$ equiv) was added dropwise to a stirred solution of $\mathbf{4 8}(0.38$ g, $1.8 \mathrm{mmol}, 1.0$ equiv) in diethyl ether $\left(3.8 \mathrm{~mL}\right.$, anhydrous, previously flushed with Ar) at $0{ }^{\circ} \mathrm{C}$. After 15 minutes $\mathrm{CO}_{2}$ was bubbled through the reaction mixture for 10 minutes. The mixture was then allowed to warm to room temperature and stirred for an additional 30 minutes. The reaction was quenched with $\mathrm{NaHCO}_{3}$ (saturated aqueous solution, $15 \mathrm{~mL})$. The aqueous layer was washed with diethyl ether $(3 \times 15 \mathrm{~mL})$, then acidified to $\mathrm{pH} 1$ with $2 \mathrm{~N} \mathrm{HCl}$ and extracted with $\mathrm{Et}_{2} \mathrm{O}(3 \times 15 \mathrm{~mL})$. The combined organic layers were dried over $\mathrm{MgSO}_{4}$, filtered and concentrated in vacuo to afford benzoic acid $5 \mathbf{d}$ (143 mg, $0.811 \mathrm{mmol}, 45 \%$ yield; 2.6 $: 1$, mixture of olefin isomers) as a colorless solid. Rf 0.23 (hexane/EtOAc 4:1). ${ }^{1} \mathrm{H}$ NMR (400 MHz, $\mathrm{CDCl}_{3}$, data for a mixture $2.6: 1$ of olefin isomers based on the relative integration of peaks at $\delta 1.79$ and 1.40) $\delta 11.2$ (bs, $1 \mathrm{H}, \mathrm{COO} H$ ), 8.03 (dd, $J=7.9,1.4 \mathrm{~Hz}, 1 \mathrm{H}, \operatorname{Ar} H$ ), 7.92 (dd, $J=7.7,1.4 \mathrm{~Hz}, 0.5 \mathrm{H}, \operatorname{Ar} H$ ), 7.54 (td, $J=7.6$, $1.4 \mathrm{~Hz}, 1 \mathrm{H}, \operatorname{Ar} H), 7.47$ (td, $J=7.6,1.5 \mathrm{~Hz}, 0.5 \mathrm{H}, \operatorname{Ar} H), 7.41-7.28$ (m, 1.5 H, $\operatorname{Ar} H), 7.22(\mathrm{dd}, J=7.7,1.2 \mathrm{~Hz}$, $0.5 \mathrm{H}, \mathrm{Ar} H), 7.15(\mathrm{dd}, J=7.7,1.2 \mathrm{~Hz}, 1 \mathrm{H}, \mathrm{Ar} H), 5.50\left(\mathrm{~m}, 1 \mathrm{H}, \mathrm{C}=C H \mathrm{CH}_{3}\right), 5.50\left(\mathrm{~m}, 0.5 \mathrm{H}, \mathrm{C}=\mathrm{CHCH}_{3}\right), 2.04$ $\left(\mathrm{m}, 3 \mathrm{H}, \mathrm{CH}_{3}\right), 1.98\left(\mathrm{~m}, 1.5 \mathrm{H}, \mathrm{CH}_{3}\right), 1.77$ (dd, $\left.J=6.7,1.0 \mathrm{~Hz}, 1.5 \mathrm{H}, \mathrm{CH}_{3}\right), 1.38(\mathrm{dd}, J=6.7,1.5 \mathrm{~Hz}, 3 \mathrm{H}$, $\left.\mathrm{CH}_{3}\right) .{ }^{1} \mathrm{H}$ NMR spectra corresponded to the literature values. ${ }^{17}$

\section{2-(2-Methylallyl)benzoic acid (5e)}

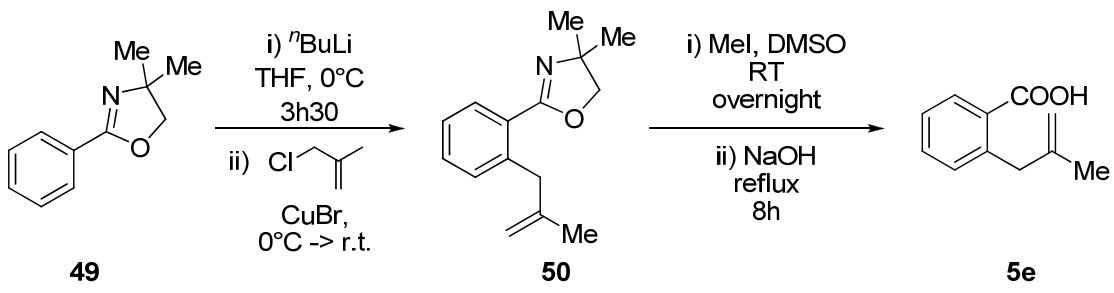


Following a reported procedure, ${ }^{21} n$-BuLi $(2.5 \mathrm{M}$ in hexanes, $6.1 \mathrm{~mL}, 15 \mathrm{mmol}, 1.3$ equiv) was added dropwise to a solution of 4,4-dimethyl-2-phenyl-2-oxazoline (49) (2.0 g, $11 \mathrm{mmol}, 1.0$ equiv) in THF (34 mL, previously flushed with argon) at $0{ }^{\circ} \mathrm{C}$. The mixture was stirred at $0^{\circ} \mathrm{C}$ for $3 \mathrm{~h} 30$ and then it was transferred to a suspension of $\mathrm{CuBr}(1.61 \mathrm{~g}, 11.2 \mathrm{mmol}, 0.99$ equiv) in THF (10 mL) via cannula. The resulting green mixture was stirred at $0{ }^{\circ} \mathrm{C}$ for $1 \mathrm{~h} 30$, methallyl chloride $(1.0 \mathrm{~mL}, 10 \mathrm{mmol}, 0.9$ equiv) was added and the reaction mixture was stirred at room temperature overnight. The reaction was then quenched by addition of water (10 $\mathrm{mL})$ and $\mathrm{NH}_{3}(25 \%$ aq. solution, $10 \mathrm{~mL})$. The aqueous layer was extracted with diethyl ether $(3 \mathrm{x} 20 \mathrm{~mL})$ and the combined organic layers were washed with brine, dried over $\mathrm{MgSO}_{4}$, filtered and concentrated under reduced pressure. Purification of the crude product by column chromatography $\left(\mathrm{SiO}_{2}\right.$, Hexane/EtOAc 10:1) afforded 4,4-dimethyl-2-(2-(2-methylallyl)phenyl)-4,5-oxazoline (50) (1.26 g, 5.49 mmol, 47 \% yield) as a dark oil.

The oxazoline was converted to the methiodide salt by stirring in excess MeI $(2.1 \mathrm{~mL}, 33 \mathrm{mmol}, 6$ equiv) and DMSO (1.2 mL) overnight at room temperature. The solvents were then evaporated in vacuo and the crude oxazoline methiodide was treated with $2 \mathrm{M} \mathrm{NaOH}(17.3 \mathrm{~mL})$ at reflux for $9 \mathrm{~h}$. The solution was then allowed to cool to room temperature and washed with DCM $(3 \times 20 \mathrm{~mL})$. The aqueous layer was acidified to $\mathrm{pH} 1 \mathrm{with} 12$ $\mathrm{N} \mathrm{HCl}$ and extracted with DCM $(3 \times 25 \mathrm{~mL})$. The combined organic layers were washed with brine, dried over $\mathrm{MgSO}_{4}$, filtered and concentrated under reduced pressure. The crude product was filtered on $\mathrm{SiO}_{2}$ to afford 2(2-methylallyl)benzoic acid (5e) (0.53 g, $3.1 \mathrm{mmol}, 55 \%$ yield) as a colorless solid. $\mathrm{R}_{\mathrm{f}} 0.42$ (28:12:1 hexaneether-formic acid). ${ }^{1} \mathrm{H} \mathrm{NMR}\left(400 \mathrm{MHz}, \mathrm{CDCl}_{3}\right) \delta 11.2(\mathrm{bs}, 1 \mathrm{H}, \mathrm{COOH}), 8.03(\mathrm{dd}, J=0.9,7.4, \mathrm{~Hz}, 1 \mathrm{H}, \mathrm{ArH})$, $7.49(\mathrm{td}, J=1.5,7.6 \mathrm{~Hz}, 1 \mathrm{H}, \mathrm{ArH}), 7.36-7.26(\mathrm{~m}, 2 \mathrm{H}, \mathrm{ArH}), 4.8\left(\mathrm{~d}, J=0.7 \mathrm{~Hz}, 1 \mathrm{H}, \mathrm{C}=\mathrm{CH}_{2}\right), 4.47(\mathrm{~d}, J=0.7$ $\left.\mathrm{Hz}, 1 \mathrm{H}, \mathrm{C}=\mathrm{CH}_{2}\right), 3.78\left(\mathrm{~s}, 2 \mathrm{H}, \mathrm{CH}_{2}\right), 1.76\left(\mathrm{~s}, 3 \mathrm{H}, \mathrm{CH}_{3}\right) .{ }^{13} \mathrm{C} \mathrm{NMR}\left(100 \mathrm{MHz}, \mathrm{CDCl}_{3}\right) \delta 173.1,145.4,142.2$, 132.7, 131.6, 131.5, 128.8, 126.3, 111.6, 41.8, 23.0. IR v 3075 (m), $2972(\mathrm{~m}), 2900(\mathrm{~m}), 2817(\mathrm{sh}), 2653(\mathrm{~m})$, 2556 (m), 1688 (s), 1656 (sh), 1602 (w), 1576 (w), 1489 (w), 1449 (m), 1406 (m), 1378 (sh), 1301 (s), 1271 (s), $1196(\mathrm{w}), 1168(\mathrm{w}), 1142(\mathrm{w}), 1083$ (w), $1051(\mathrm{w}), 1018(\mathrm{w}), 891(\mathrm{~s}), 847(\mathrm{w}), 802$ (m), 783 (s), 740 (s), 709 $(\mathrm{w}), 658(\mathrm{~m})$. Characterization data for 5 e corresponded to the literature values. ${ }^{22}$

\section{$N$-(Benzyloxy)-2-(prop-2-en-1-yl)benzamide (7a)}

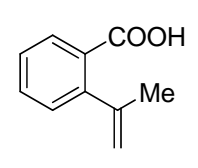

$5 \mathbf{a}$

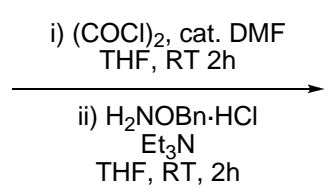

THF, RT, 2h

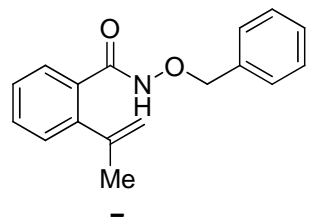

$7 \mathbf{a}$

Following a reported procedure, ${ }^{17}$ oxalyl chloride $(1.3 \mathrm{~mL}, 15 \mathrm{mmol}, 5.0$ equiv) was added to a stirred solution of acid 5a (487 mg, $3.00 \mathrm{mmol}, 1.00$ equiv) in THF (16 mL), followed by a catalytic amount of DMF 
(3 drops). After $2 \mathrm{~h}$ the solvent were evaporated under reduced pressure. The residue was diluted with THF (16 $\mathrm{mL}$ ) and reacted with $O$-benzylhydroxylamine-hydrochloride $(957 \mathrm{mg}, 5.99 \mathrm{mmol}, 2.00$ equiv) and triethylamine $(2.1 \mathrm{~mL}, 15 \mathrm{mmol}, 5.0$ equiv). The mixture was stirred for $2 \mathrm{~h}$ and then quenched by adding $2 \mathrm{M}$ $\mathrm{NaOH}(24 \mathrm{~mL})$. The aqueous layer was extracted with EtOAc $(3 \times 25 \mathrm{~mL})$. The combined organic layers were washed with $2 \mathrm{M} \mathrm{HCl}(25 \mathrm{~mL})$, dried over $\mathrm{MgSO}_{4}$, filtered and the solvent was evaporated in vacuo. Purification by column chromatography ( $\mathrm{SiO}_{2}$, PET/EtOAc 4:1) afforded $N$-(benzyloxy)-2-(prop-2-en-1yl)benzamide (7a) (635 mg, $2.37 \mathrm{mmol}, 79 \%$ yield) as a pale yellow solid. $\mathrm{R}_{\mathrm{f}} 0.15$ (Hexane/EtOAc 1:1). Mp 84 $-88^{\circ} \mathrm{C} .{ }^{1} \mathrm{H}$ NMR $\left(400 \mathrm{MHz}, \mathrm{CDCl}_{3}\right) \delta 8.92$ (bs, $\left.1 \mathrm{H}, \mathrm{NH}\right), 7.50$ (d, J=7.4 Hz, $\left.1 \mathrm{H}, \mathrm{ArH}\right), 7.47-7.31$ (m, $6 \mathrm{H}, 5$ $\mathrm{H} \mathrm{PhH}$ and $1 \mathrm{H}, \mathrm{Ar} H), 7.27(\mathrm{td}, J=7.6,1.2 \mathrm{~Hz}, 1 \mathrm{H}, \mathrm{ArH}), 7.20(\mathrm{~d}, J=7.6 \mathrm{~Hz}, 1 \mathrm{H}, \mathrm{ArH}), 5.12(\mathrm{t}, J=1.5 \mathrm{~Hz}, 1$ $\left.\mathrm{H}, \mathrm{C}=\mathrm{CCH}_{2}\right), 5.05-4.95\left(\mathrm{~m}, 3 \mathrm{H}, 1 \mathrm{H}, \mathrm{C}=\mathrm{CCH}_{2}\right.$ and $\left.2 \mathrm{H}, \mathrm{PhCH}_{2}\right), 2.01\left(\mathrm{~m}, 3 \mathrm{H}, \mathrm{CH}_{3}\right) .{ }^{13} \mathrm{C} \mathrm{NMR}(100 \mathrm{MHz}$, $\left.\mathrm{CDCl}_{3}\right) \oint 167.1,145.1,142.0,135.3,130.9,130.5,128.8,128.7,128.5,128.4,127.2,116.1,77.2,24.0$ (two carbons cannot be resolved). IR v 3188 (m), $3087(\mathrm{~m}), 3064(\mathrm{~m}), 3031$ (m), $2966(\mathrm{~m}), 2948$ (m), 2876 (m), 2349 (w), 1650 (s), 1598 (m), 1570 (w), 1497 (m), 1455 (m), 1371 (m), 1303 (m), 1212 (w), 1161 (w), 1088 (w), 1021 (s), 955 (w), 901 (s), 837 (w), 819 (w), 770 (s), 745 (s), 699 (s), 660 (m). HRMS (ESI) calcd for $\mathrm{C}_{17} \mathrm{H}_{18} \mathrm{NO}_{2}(\mathrm{M}+\mathrm{H})$ 268.1338; found 268.1328.

\section{2,2,4-Trimethylpent-4-enoic acid (9a)}

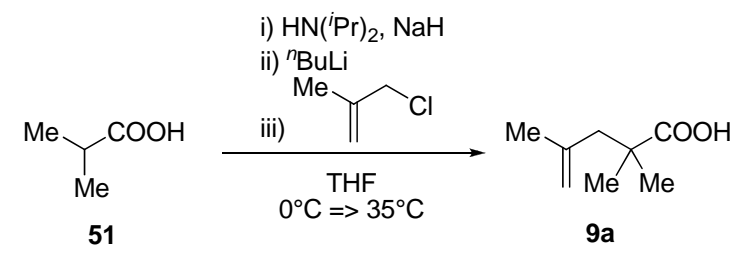

Following a reported procedure, ${ }^{23}$ isobutyric acid (51) (475 mg, 5.39 mmol, 1.0 equiv) was added dropwise to a stirred suspension of $\mathrm{NaH}$ (60\% dispersion in mineral oil) (236 mg, $5.89 \mathrm{mmol}, 1.1$ equiv) and diisopropylamine $(0.78 \mathrm{~mL}, 5.5 \mathrm{mmol}, 1.0$ equiv) in THF $(7.5 \mathrm{~mL})$. The resulting suspension was heated at reflux for $20 \mathrm{~min}$ and then cooled to $0^{\circ} \mathrm{C}$ for $15 \mathrm{~min}$ prior to the dropwise addition of ${ }^{n} \mathrm{BuLi}(2.5 \mathrm{M}$ in hexanes) (2.2 $\mathrm{mL}, 5.4 \mathrm{mmol}, 1.0$ equiv). The resulting greenish suspension was stirred at $0^{\circ} \mathrm{C}$ for an additional $15 \mathrm{~min}$ and then heated to $35^{\circ} \mathrm{C}$ for $30 \mathrm{~min}$. Then it was cooled to $0^{\circ} \mathrm{C}$ and methallyl chloride $(0.54 \mathrm{~mL}, 5.5 \mathrm{mmol}, 1.0$ equiv) was added dropwise to give an off-white suspension which was stirred for $2 \mathrm{~h}$ at $35^{\circ} \mathrm{C}$. The suspension was then cooled with an ice-bath and the excess of $\mathrm{NaH}$ was neutralized with water $(10 \mathrm{~mL})$. The organic layer was washed with a $1 \mathrm{M} \mathrm{NaOH}$ solution $(3 \times 20 \mathrm{~mL})$ and the combined aqueous layers were then extracted with $\mathrm{Et}_{2} \mathrm{O}(20 \mathrm{~mL})$. The aqueous layer was acidified by addition of a $1 \mathrm{M} \mathrm{HCl}$ solution until $\mathrm{pH} 3$ and was then extracted with ether $(3 \times 20 \mathrm{~mL})$. The combined organic layers were dried over $\mathrm{MgSO}_{4}$, filtered and the solvent was evaporated in vacuo to afford 2,2,4-trimethylpent-4-enoic acid (9a) (611 mg, $4.29 \mathrm{mmol}, 79 \%$ yield) as a colorless oil. $\mathrm{R}_{\mathrm{f}} 0.61$ (Petroleum Ether/EtOAc 5/1). ${ }^{1} \mathrm{H}$ NMR (400 MHz, $\mathrm{CDCl}_{3}$ ) $\delta 9.91$ (br s, $\left.1 \mathrm{H}, \mathrm{COOH}\right), 4.83$ 
$\left(\mathrm{m}, 1 \mathrm{H}, \mathrm{C}=\mathrm{CH}_{2}\right), 4.70\left(\mathrm{~m}, 1 \mathrm{H}, \mathrm{C}=\mathrm{CH}_{2}\right), 2.34\left(\mathrm{~d}, \mathrm{~J}=0.5 \mathrm{~Hz}, 2 \mathrm{H}, \mathrm{CH}_{2}\right), 1.71\left(\mathrm{~s}, 3 \mathrm{H}, \mathrm{CH}_{3} \mathrm{C}=\mathrm{C}\right), 1.24(\mathrm{~s}, 6 \mathrm{H}$, $\left.\mathrm{C}\left(\mathrm{CH}_{3}\right)_{2}\right) .{ }^{13} \mathrm{C} \mathrm{NMR}\left(100 \mathrm{MHz}, \mathrm{CDCl}_{3}\right) \delta 185.2,142.2,114.4,48.2,42.0,25.3,23.6 . \mathrm{IR} v 3126(\mathrm{~m}), 3077(\mathrm{~m})$, 2976 (m), 2933 (m), 2883 (m), 2685 (w), 2619 (w), 1700 (s), 1647 (w), 1475 (m), 1453 (m), 1411 (m), 1371 (w), 1314 (m), 1281 (m), 1225 (m), 1165 (w), 1139 (w), 1021 (w), 943 (m), 897 (s), 870 (m), 829 (w), $811(\mathrm{w})$, $790(w), 767(w)$. Characterization data for 9a corresponded to the literature values. ${ }^{22}$

\section{4-Methylpent-4-enoic acid (9b)}

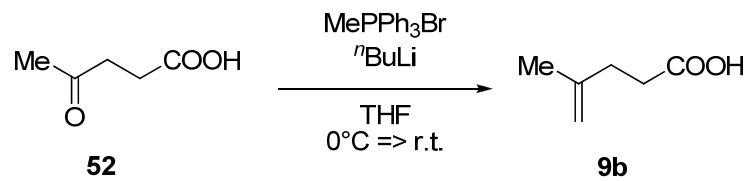

Following a reported procedure, ${ }^{24}$ methyltriphenylphosphonium bromide $(3.75 \mathrm{~g}, 10.5 \mathrm{mmol}, 3.0$ equiv) was suspended in THF (46 mL). The colorless suspension was cooled to $0^{\circ} \mathrm{C}$ and ${ }^{n} \mathrm{BuLi}(2.5 \mathrm{M}$ in hexanes) (4.2 $\mathrm{mL}$, $10.5 \mathrm{mmol}, 3.0$ equiv) was added dropwise. The resulting bright orange solution was stirred at $0^{\circ} \mathrm{C}$ for $1 \mathrm{~h}$ before the dropwise addition of levulinic acid (52) $(0.41 \mathrm{~g}, 3.5 \mathrm{mmol}, 1.0$ equiv). The suspension was allowed to warm to room temperature and stirred overnight. The reaction was then quench by adding a $1 \mathrm{M} \mathrm{HCl}$ solution $(10 \mathrm{~mL})$ and the aqueous layer was extracted with $\mathrm{Et}_{2} \mathrm{O}(3 \times 40 \mathrm{~mL})$. The combined organic layers were dried over $\mathrm{MgSO}_{4}$, filtered and concentrated under reduced pressure. The crude product was purified by column chromatography $\left(\mathrm{SiO}_{2}\right.$, Petroleum ether/EtOAc/AcOH 85: 15: 0.1) to afford 4-methylpent-4-enoic acid (9b) (247 mg, 2.16 mmol, 62\% yield) as a clear yellowish oil. $\mathrm{R}_{\mathrm{f}} 0.47$ (Petroleum Ether/EtOAc 4/1). ${ }^{1} \mathrm{H}$ NMR (400 $\left.\mathrm{MHz}_{2} \mathrm{CDCl}_{3}\right) \delta 9.74($ br s, $1 \mathrm{H}, \mathrm{COOH}), 4.77\left(\mathrm{~m}, 1 \mathrm{H}, \mathrm{C}=\mathrm{CH}_{2}\right), 4.71\left(\mathrm{~d}, J=0.7 \mathrm{~Hz}, 1 \mathrm{H}, \mathrm{C}=\mathrm{CH}_{2}\right), 2.54(\mathrm{~m}, 2 \mathrm{H}$, $\left.\mathrm{CH}_{2} \mathrm{COOH}\right), 2.37\left(\mathrm{t}, \mathrm{J}=8.2 \mathrm{~Hz}, \mathrm{CH}_{2} \mathrm{C}=\mathrm{CH}_{2}\right), 1.75\left(\mathrm{~d}, \mathrm{~J}=0.4 \mathrm{~Hz}, 3 \mathrm{H}, \mathrm{CH}_{3} \mathrm{C}=\mathrm{CH}_{2}\right) .{ }^{13} \mathrm{C} \mathrm{NMR}(100 \mathrm{MHz}$, $\left.\mathrm{CDCl}_{3}\right) \delta 180.0,143.7,110.5$, 32.4, 32.2, 22.5. IR v $3080(\mathrm{~m}), 3034$ (m), $2974(\mathrm{~m}), 2917(\mathrm{~m}), 2857$ (m), 2682 (w), 1710 (s), 1652 (w), 1444 (m), 1416 (m), 1379 (w), 1293 (m), 1245 (m), 1212 (m), 1164 (w), 1051 (w), 935 (w, sh), 891 (s), 841 (w), 787 (w). Characterization data for $9 \mathbf{b}$ corresponded to the literature values. ${ }^{25}$

\section{2,2-Dimethylpent-4-enoic acid (9c)}

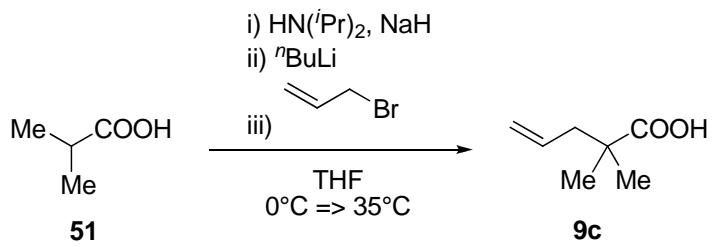

Following a reported procedure, ${ }^{22}$ isobutyric acid (51) (475 mg, $5.39 \mathrm{mmol}, 1.0$ equiv) was added dropwise to a stirred suspension of $\mathrm{NaH}(60 \%$ dispersion in mineral oil) (236 $\mathrm{mg}, 5.89 \mathrm{mmol}, 1.1$ equiv) and diisopropylamine $(0.78 \mathrm{~mL}, 5.5 \mathrm{mmol}, 1.0$ equiv) in THF $(7.5 \mathrm{~mL})$. The resulting suspension was heated at

(24) Lee, E. E.; Rovis, T. Org. Lett., 2008, 10, 1231.

(25) Braddock, D. C.; Cansell, G.; Hermitage, S. A. Chem. Commun., 2006, 2483. 
reflux for $20 \mathrm{~min}$ and then cooled to $0^{\circ} \mathrm{C}$ for $15 \mathrm{~min}$ prior to the dropwise addition of ${ }^{n} \mathrm{BuLi}(2.5 \mathrm{M}$ in hexanes) (2.2 $\mathrm{mL}, 5.4 \mathrm{mmol}, 1.0$ equiv). The resulting greenish suspension was stirred at $0^{\circ} \mathrm{C}$ for an additional $15 \mathrm{~min}$ and then heated to $35^{\circ} \mathrm{C}$ for $30 \mathrm{~min}$. Passed this time it was cooled to $0^{\circ} \mathrm{C}$ and allyl bromide $(0.48 \mathrm{~mL}, 5.5$ mmol, 1.0 equiv) was added dropwise to give an off-white suspension which was stirred for $2 \mathrm{~h}$ at $35^{\circ} \mathrm{C}$. The suspension was then cooled with an ice-bath and the excess of $\mathrm{NaH}$ was neutralized with water $(10 \mathrm{~mL})$. The organic layer was washed with a $1 \mathrm{M} \mathrm{NaOH}$ solution $(3 \times 20 \mathrm{~mL})$ and the combined aqueous layers were then extracted with $\mathrm{Et}_{2} \mathrm{O}(20 \mathrm{~mL})$. The aqueous layer was acidified by addition of a $1 \mathrm{M} \mathrm{HCl}$ solution until $\mathrm{pH} 3$ and it was then extracted with ether $(3 \times 20 \mathrm{~mL})$. The combined organic layers were dried over $\mathrm{MgSO}_{4}$, filtered and the solvent was evaporated in vacuo. The crude product was purified by column chromatography $\left(\mathrm{SiO}_{2}\right.$, Petroleum ether/EtOAc/AcOH 95: 5: 0.1) to afford 2,2-dimethylpent-4-enoic acid (9c) (150 mg, $1.17 \mathrm{mmol}$, 22\%). $\mathrm{R}_{\mathrm{f}} 0.56$ (Petroleum ether/EtOAc/AcOH 80: 20: 0.1). ${ }^{1} \mathrm{H}$ NMR (400 MHz, $\left.\mathrm{CDCl}_{3}\right) \delta 8.80($ br s, $1 \mathrm{H}$, $\mathrm{COOH}), 5.77\left(\mathrm{~m}, 1 \mathrm{H}, \mathrm{CH}=\mathrm{CH}_{2}\right), 5.10\left(\mathrm{~m}, 1 \mathrm{H}, \mathrm{CH}=\mathrm{CH}_{2}\right), 5.06\left(\mathrm{~m}, 1 \mathrm{H}, \mathrm{CH}=\mathrm{CH}_{2}\right), 2.31(\mathrm{dt}, J=7.4,1.0 \mathrm{~Hz}, 2$ $\left.\mathrm{H}, \mathrm{CH}_{2} \mathrm{CH}=\mathrm{CH}_{2}\right), 1.20\left(\mathrm{~s}, 6 \mathrm{H}, \mathrm{C}\left(\mathrm{CH}_{3}\right)_{2}\right) .{ }^{13} \mathrm{C} \mathrm{NMR}\left(100 \mathrm{MHz}, \mathrm{CDCl}_{3}\right) \delta 184.6,133.9,118.2,44.4,41.5,24.5$. IR v $3078(\mathrm{~m}), 2979(\mathrm{~m}), 2930(\mathrm{~m}), 2879(\mathrm{~m}), 2714(\mathrm{w}), 1702$ (s), 1643 (w), $1475(\mathrm{w}), 1411(\mathrm{w}), 1388(\mathrm{w})$, 1367 (w), 1315 (w), 1238 (w), 1207 (w), 1181 (w), 997 (w), 919 (m), 863 (w), 739 (w), 677 (w), 655 (w), 645 (w), $624(\mathrm{w}), 616(\mathrm{w})$. Characterization data for 9c corresponded to the literature values. ${ }^{26}$

\section{Optimization of the Reaction}

\section{General procedure for reaction optimization:}

The catalyst ( $0.014 \mathrm{mmol}, 0.20$ equiv) was dissolved in the dry solvent $(5 \mathrm{~mL})$ and the mixture was stirred under nitrogen. Methylallyl phenol (1a) $(10 \mathrm{mg}, 0.069 \mathrm{mmol}, 1.0$ equiv) was then added, followed by the hypervalent iodine reagent $(35 \mathrm{mg}, 0.084 \mathrm{mmol}, 1.2$ equiv). The mixture was stirred at room temperature overnight. It was then filtered over silica gel and the filtrate was concentrated in vacuo. The residue was diluted with dichloromethane $(1 \mathrm{~mL}$, solution A). $0.1 \mathrm{~mL}$ of a solution of decane $0.02 \mathrm{M}$ in dichloromethane and $0.8 \mathrm{~mL}$ of dichloromethane were added to $0.1 \mathrm{~mL}$ of solution A. The resulting solution was injected into GC-MS and the following oven program was followed: Initial temperature: $50 \mathrm{~min}$, Ramp: $10.0{ }^{\circ} \mathrm{C} / \mathrm{min}$ to $250{ }^{\circ} \mathrm{C}$, hold 15 $\min$ at $250^{\circ} \mathrm{C}$.

\section{GC-MS Quantification}

A solution of decane $\mathrm{C}=0.02 \mathrm{M}$ in dichloromethane and a solution of product $4 \mathrm{C}=0.02 \mathrm{M}$ in dichloromethane were prepared. For the calibration, 3 points were measured corresponding to 3 different ratio (decane : 4). The ratios used are 1:1,1:2 and 2:1.

The observed ratio by integration of the GC peaks and the real ratios were used as the axis of the calibration graph (Figure S1 for the example of $\mathbf{4 b}$ ). 


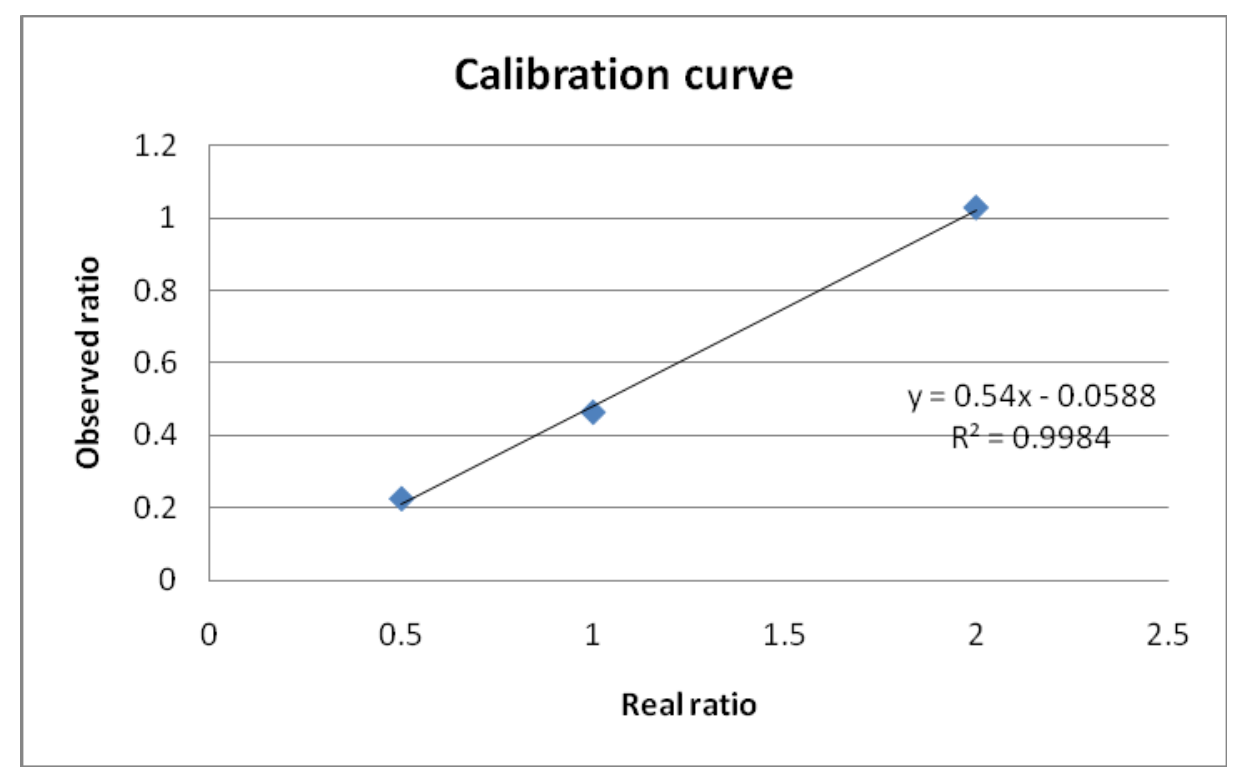

Figure S1: Calibration curve for GC yields calculation in the case of $\mathbf{4 b}$. 
Detailed results for the optimization studies

Table S1: Catalyst screening for the reaction of 1 a with $\mathbf{3 d}$.

\begin{tabular}{|c|c|c|}
\hline Entry & Catalyst & Yield $^{\mathrm{a}}$ \\
\hline 1 & $\operatorname{Pd}(\mathrm{TFA})_{2}$ & $17 \%$ \\
\hline 2 & $\mathrm{Pd}(\mathrm{OAc})_{2}$ & $18 \%$ \\
\hline 3 & $\mathrm{PdCl}_{2}\left(\mathrm{CH}_{3} \mathrm{CN}\right)_{2}$ & $40 \%$ \\
\hline 4 & {$\left[\mathrm{Pd}(\mathrm{MeCN})_{4}\right]\left(\mathrm{BF}_{4}\right)_{2}$} & $21 \%$ \\
\hline 5 & $\operatorname{Pd}(\mathrm{acac})_{2}$ & $2 \%$ \\
\hline 6 & $\operatorname{Pd}(\text { hfacac })_{2}$ & $73 \%$ \\
\hline 7 & $\mathrm{PdCl}_{2}\left(\mathrm{PPh}_{3}\right)_{2}$ & traces \\
\hline 8 & $\mathrm{Pd}(\text { hfacac })_{2} / \mathrm{PPh}_{3}$ & traces \\
\hline 9 & & $0 \%$ \\
\hline 10 & & $0 \%$ \\
\hline 11 & & $31 \%$ \\
\hline 12 & $\operatorname{Pd}(\mathrm{dba})_{2}$ & $7 \%$ \\
\hline 13 & $\mathrm{Pd}_{2}(\mathrm{dba})_{3} \cdot \mathrm{CHCl}_{3}$ & $6 \%$ \\
\hline 14 & $10 \mathrm{~mol} \% \mathrm{Pd}(\mathrm{hfacac})_{2}$ & $71 \%$ \\
\hline 15 & $5 \mathrm{~mol} \% \mathrm{Pd}(\mathrm{hfacac})_{2}$ & $68 \%$ \\
\hline 16 & $40 \mathrm{~mol} \% \mathrm{Pd}(\mathrm{hfacac})_{2}$ & $79 \%$ \\
\hline
\end{tabular}

${ }^{\mathrm{a}}$ Reaction conditions: $0.069 \mathrm{mmol}$ 1a, 20\% mol catalyst, 1.2 equiv reagent, 5 $\mathrm{mL}$ dry solvent under $\mathrm{N}_{2}$. Yield was determined via GC-MS. 
Table S2: Solvents screening for the reaction of 1a with 3d.

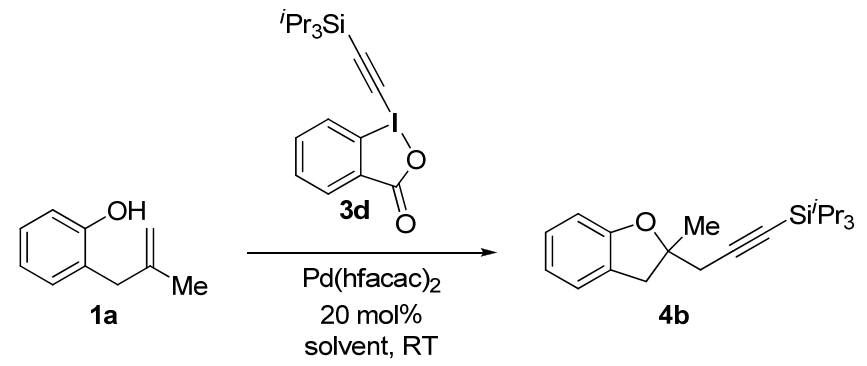

\begin{tabular}{ccc}
\hline Entry & Solvent & Yield $^{\mathrm{a}}$ \\
\hline $\mathbf{1}$ & $\mathrm{CH}_{2} \mathrm{Cl}_{2}$ & $73 \%$ \\
$\mathbf{2}$ & $\mathrm{CHCl}_{3}$ & $70 \%$ \\
$\mathbf{3}$ & $\mathrm{C}_{2} \mathrm{H}_{4} \mathrm{Cl}_{2}$ & $67 \%$ \\
$\mathbf{4}$ & toluene & $32 \%$ \\
$\mathbf{5}$ & hexane/toluene $1: 1$ & $42 \%$ \\
$\mathbf{6}$ & $\alpha, \alpha, \alpha$-trifluorotoluene & $67 \%$ \\
$\mathbf{7}$ & $\mathrm{THF}$ & $12 \%$ \\
$\mathbf{8}$ & Et & $9 \%$ \\
$\mathbf{9}$ & acetone & $9 \%$ \\
$\mathbf{1 0}$ & $\mathrm{CH}_{3} \mathrm{CN}$ & $21 \%$ \\
$\mathbf{1 1}$ & methanol & $25 \%$ \\
$\mathbf{1 2}$ & DMF & traces \\
\hline${ }^{\mathrm{R}}$ & &
\end{tabular}

${ }^{\mathrm{a}}$ Reaction conditions: $0.069 \mathrm{mmol}$ 1a, $20 \%$ mol catalyst, 1.2 equiv reagent, $5 \mathrm{~mL}$ dry solvent under $\mathrm{N}_{2}$. Yield was determined via GC-MS.

\section{Scope of the Reaction}

Trimethyl-[3-(2-methyl-2,3-dihydro-benzofuran-2-yl)-prop-1-ynyl]-silane (4a)

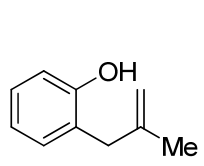

$1 \mathbf{a}$

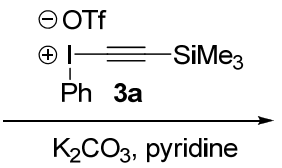

toluene

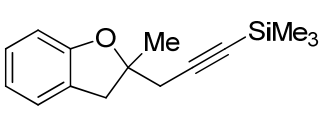

$4 a$

A small amount of 4a was obtained using the conditions in entry 1, Table 1 on a preparative scale: 2(methylallyl)phenol (1a) (85 mg, $0.57 \mathrm{mmol}, 1.0$ equiv) was added to a solution of $\mathrm{Pd}(\mathrm{II})$ bis(acetonitrile) (18 $\mathrm{mg}, 0.057 \mathrm{mmol}, 0.10$ equiv), pyridine $(9 \mu \mathrm{L}, 0.1 \mathrm{mmol}, 0.2$ equiv) and potassium carbonate (158 $\mathrm{mg}, 1.14$ mmol, 2.00 equiv) in toluene $(4.6 \mathrm{~mL})$. Phenyl(trimethylsilylethynyl)iodonium triflate (4a) (308 mg, 0.685 mmol, 1.2 equiv) was then added and the mixture was stirred at $0{ }^{\circ} \mathrm{C}$. After $1 \mathrm{~h}$, the mixture was warmed to room temperature and stirred additionally $90 \mathrm{~min}$. Then a solution of $\mathrm{NaHCO}_{3}$ sat. was added, followed by water and a solution of $\mathrm{NaCl}$ sat., followed by an extraction with diethyl ether $(3 \times 20 \mathrm{ml})$. It was then dried with 
$\mathrm{MgSO}_{4}$ and evaporated under reduced pressure.The black crude oil was purified by chromatography (hexane $\left./ \mathrm{CH}_{2} \mathrm{Cl}_{2} 4: 1\right)$ to yield $\mathbf{4 a}(8.5 \mathrm{mg}, 0.035 \mathrm{mmol}, 6 \%)$ as a yellow oil, which was used for GC/MS calibration. $\mathrm{R}_{\mathrm{f}} 0.56$ (hexane/EtOAc 10:1). ${ }^{1} \mathrm{H}$ NMR $\left(400 \mathrm{MHz}, \mathrm{CDCl}_{3}\right) \delta 7.12(\mathrm{~m}, 2 \mathrm{H}, \mathrm{Ar} \mathrm{H}), 6.83(\mathrm{t}, J=7.4$ $\mathrm{Hz}, 1 \mathrm{H}, \mathrm{Ar} \mathrm{H}), 6.74$ (d, J=8.0 Hz, $1 \mathrm{H}, \mathrm{Ar} \mathrm{H}), 3.31\left(\mathrm{~m}, 1 \mathrm{H}, \mathrm{CH}_{2}\right), 3.00$ (d, J=15.7 Hz, $\left.1 \mathrm{H}, \mathrm{CH}_{2}\right), 2.62(\mathrm{~m}, 2$ $\mathrm{H}, \mathrm{CH}_{2}$ ), $1.58(\mathrm{~s}, 3 \mathrm{H}, \mathrm{Me}), 0.11(\mathrm{~s}, 9 \mathrm{H}, \mathrm{TMS})$. MS (EI) found for $\mathrm{C}_{15} \mathrm{H}_{20} \mathrm{OSi}^{+}$(M) 244. GC Elution Time: 18.7 min.Due to the low amount of product 4a and the low yield generally obtained, no further characterization was made.

\section{General optimized procedure for the Wacker cyclization - alkynylation domino reaction:}

Palladium (II) hexafluoroacetylacetonate $(21 \mathrm{mg}, 0.10$ equiv.) was dissolved in dichloromethane $(10 \mathrm{~mL})$. The substrate $(0.40 \mathrm{mmol})$ was then added, followed by 1-((triisopropylsilyl)ethynyl)-1,2-benziodoxol-3(1 H)one (3d). The resulting solution was stirred under $\mathrm{N}_{2}$ at room temperature for $5 \mathrm{~h}$. DCM was then removed under reduced pressure and the residue was treated with $\mathrm{NaHCO}_{3}$ (saturated solution, $6 \mathrm{~mL}$ ) and brine $(6 \mathrm{~mL})$. The mixture was extracted with diethyl ether $(3 \times 15 \mathrm{~mL})$ and the combined organic layers were dried on $\mathrm{MgSO}_{4}$, filtered and concentrated in vacuo. The brown crude oil was purified by column chromatography on silica gel.

Triisopropyl-[3-(2-methyl-2,3-dihydro-benzofuran-2-yl)-prop-1-ynyl]-silane (4b)

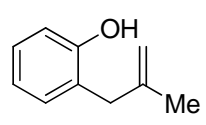

$1 \mathbf{a}$

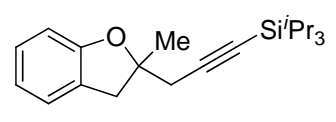

$\mathbf{4 b}$

Column chromatography ( $\mathrm{SiO}_{2}, \mathrm{PET} / \mathrm{DCM}$ 98:2) afforded product $\mathbf{4 b}$ (92 $\mathrm{mg}, 0.28 \mathrm{mmol}, 71 \%$ yield) as a yellow oil. $\mathrm{R}_{\mathrm{f}} 0.41$ (PET/DCM 4:1). ${ }^{1} \mathrm{H}$ NMR (400 MHz, $\left.\mathrm{CDCl}_{3}\right) \delta 7.15$ (dd, J=7.4, 0.7 Hz, $\left.1 \mathrm{H}, \mathrm{ArH}\right), 7.11$ $(\mathrm{m}, 1 \mathrm{H}, \operatorname{Ar} H), 6.83(\mathrm{td}, J=7.4,0.9 \mathrm{~Hz}, 1 \mathrm{H}, \mathrm{Ar} H), 6.74(\mathrm{~d}, J=7.9 \mathrm{~Hz}, 1 \mathrm{H}, \operatorname{Ar} H), 3.39(\mathrm{~d}, J=15.8 \mathrm{~Hz}, 1 \mathrm{H}$, $\left.\mathrm{ArCH}_{2}\right), 3.01(\mathrm{~d}, \mathrm{~J}=15.8 \mathrm{~Hz}, 1 \mathrm{H}, \mathrm{ArCH}), 2.67\left(\mathrm{~m}, 2 \mathrm{H}, \mathrm{CH}_{2} \mathrm{C} \equiv \mathrm{C}\right), 1.60$ (s, $\left.3 \mathrm{H}, \mathrm{CH}_{3}\right), 1.05$ (m, $\left.21 \mathrm{H}, \mathrm{TIPS}\right)$. ${ }^{13} \mathrm{C}$ NMR $\left(100 \mathrm{MHz} \mathrm{CDCl}_{3}\right) \delta 158.7,128.0,126.5,125.1,120.2,109.5,104.3,87.3,82.9,40.6,32.5,26.1$, 18.6, 11.2. IR v $2956(\mathrm{~m}), 2942(\mathrm{~m}), 2892(\mathrm{~m}), 2864(\mathrm{~m}), 2176(\mathrm{~m}), 1600(\mathrm{w}), 1481(\mathrm{~s}), 1462$ (m), $1380(\mathrm{w})$, 1328 (w), 1239 (m), 1116 (w), 1069 (m), 1033 (m), 1017 (m), 996 (m), 921 (m), 883 (s), 796 (w), 785 (w), 747 (s), $710(\mathrm{w}), 677$ (s), 660 (s), 636 (s). HRMS (ESI) calcd for $\mathrm{C}_{21} \mathrm{H}_{32} \mathrm{OSiNa}^{+}(\mathrm{M}+\mathrm{Na})$ 351.2120; found: 351.2139. GC Elution Time: 27.5 min.

Triethyl-[3-(2-methyl-2,3-dihydro-benzofuran-2-yl)-prop-1-ynyl]-silane (4c) 


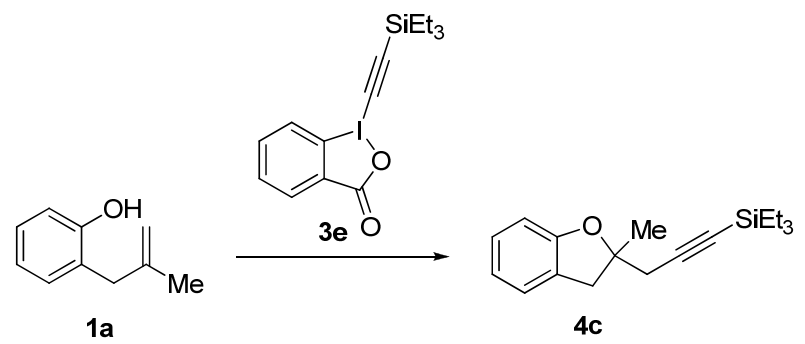

Following the general procedure, but using reagent $3 \mathbf{e}(0.19 \mathrm{~g}, 0.48 \mathrm{mmol}, 1.2$ equiv) product $4 \mathbf{c}(50 \mathrm{mg}$, 0.17 mmoles, $43 \%$ yield) was obtained as a yellow oil after purification by column chromatography $\left(\mathrm{SiO}_{2}\right.$, PET/DCM 98:2). $\mathrm{R}_{\mathrm{f}} 0.82$ (PET/EtOAc 10:1). ${ }^{1} \mathrm{H}$ NMR (400 MHz, CDCl $\left.{ }_{3}\right) \delta 7.13$ (t, J= 7.2 Hz, $\left.1 \mathrm{H}, \mathrm{ArH}\right), 7.09$ $(\mathrm{d}, J=7.4 \mathrm{~Hz}, 1 \mathrm{H}, \operatorname{Ar} H), 6.83(\mathrm{t}, J=7.4,1 \mathrm{H}, \operatorname{Ar} H), 6.73(\mathrm{~d}, J=7.9 \mathrm{~Hz}, 1 \mathrm{H}, \operatorname{Ar} H), 3.35(\mathrm{~d}, J=15.8 \mathrm{~Hz}, 1 \mathrm{H}$, $\left.\mathrm{ArCH}_{2}\right), 3.00\left(\mathrm{~d}, J=15.8 \mathrm{~Hz}, 1 \mathrm{H}, \mathrm{ArCH}_{2}\right), 2.68\left(\mathrm{~d}, J=16.7 \mathrm{~Hz}, 1 \mathrm{H}, \mathrm{CH}_{2} \mathrm{C} \equiv \mathrm{C}\right), 2.60(\mathrm{~d}, J=16.7 \mathrm{~Hz}, 1 \mathrm{H}$, $\left.\mathrm{CH}_{2} \mathrm{C} \equiv \mathrm{C}\right), 1.58\left(\mathrm{~s}, 3 \mathrm{H}, \mathrm{CH}_{3}\right), 0.95\left(\mathrm{t}, J=7.9 \mathrm{~Hz}, 9 \mathrm{H}, \mathrm{SiCH}_{2} \mathrm{CH}_{3}\right), 0.54$ (q, $\left.J=\mathrm{Hz}, 6 \mathrm{H}, \mathrm{SiCH}_{2} \mathrm{CH}_{3}\right) .{ }^{13} \mathrm{C} \mathrm{NMR}$ $\left(100 \mathrm{MHz}, \mathrm{CDCl}_{3}\right) \delta 158.7,128.0,126.6,125.1,120.3,109.5,103.8,87.3,84.3,40.6,32.5,26.1,7.5,4.5 . \mathrm{IR} v$ 3093 (s), 2956 (m), 2933 (m), 2910 (w), 2878 (m), 2349 (w), 2177 (s), 1598 (m), $1481(\mathrm{w}), 1461$ (w), $1416(\mathrm{w})$, 1379 (s), 1325 (w), 1240 (s), 1116 (m), $1116(w), 1070(w), 1034(\mathrm{~m}), 1017(\mathrm{~m}), 969$ (w), $954(\mathrm{w}), 925$ (w), 887 (w), $861(w), 792(w), 743$ (s), 632 (w). HRMS (ESI) calcd for $\mathrm{C}_{18} \mathrm{H}_{26} \mathrm{OSi}^{+}$(M+H) 287.1831; found: 287.1833.

\section{Triisopropyl-[3-(2,5-dimethyl-2,3-dihydro-benzofuran-2-yl)-prop-1-ynyl]-silane (4d)}

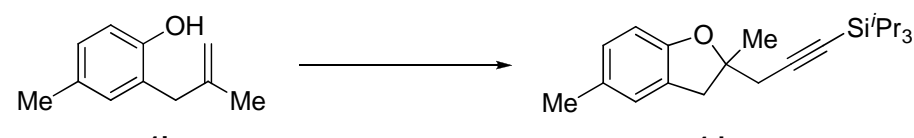

$1 \mathrm{~b}$

4d

Column chromatography ( $\left.\mathrm{SiO}_{2}, \mathrm{PET} / \mathrm{DCM} 98: 2\right)$ afforded product 4d (48 $\mathrm{mg}, 0.13 \mathrm{mmol}, 34 \%$ yield) as a yellow oil. $\mathrm{R}_{\mathrm{f}} 0.80(\mathrm{PET} / \mathrm{EtOAc} 10: 1) .{ }^{1} \mathrm{H} \mathrm{NMR}\left(400 \mathrm{MHz}, \mathrm{CDCl}_{3}\right) \delta 6.97(\mathrm{~s}, 1 \mathrm{H}, \mathrm{ArH}), 6.90(\mathrm{dd}, J=8.1,1.0$ $\mathrm{Hz}, 1 \mathrm{H}, \operatorname{Ar} H), 6.63(\mathrm{~d}, J=8.1 \mathrm{~Hz}, 1 \mathrm{H}, \mathrm{ArH}), 3.35$ (d, J=15.6 Hz, $1 \mathrm{H}, \operatorname{ArCH}), 2.97$ (d, J=15.6 Hz, $1 \mathrm{H}$, $\left.\mathrm{ArCH}_{2}\right), 2.65\left(\mathrm{~m}, 2 \mathrm{H}, \mathrm{CH}_{2} \mathrm{C} \equiv \mathrm{C}\right), 2.28\left(\mathrm{~s}, 3 \mathrm{H}, \mathrm{CH}_{3}\right), 1.59\left(\mathrm{~s}, 3 \mathrm{H}, \mathrm{CH}_{3}\right), 1.09-0.99$ (m, $\left.21 \mathrm{H}, \mathrm{TIPS}\right) .{ }^{13} \mathrm{C} \mathrm{NMR}$ $\left(100 \mathrm{MHz} \mathrm{CDCl}_{3}\right) \delta 156.6,129.5,128.3,126.6,125.7,109.0,104.5,87.4,82.8,40.7,32.5,29.8,26.1,18.7$, 11.2. IR v 2941 (s), 2864 (s), 2175 (s), 1618 (w), 1491 (s), 1464 (s), 1380 (m), 1306 (m), 1249 (s), 1217 (m), 1125 (w), 1070 (s), 1034 (s), 995 (m), 923 (m), 882 (s), 811 (s), 676 (s), 634 (m). HRMS (ESI) calcd for $\mathrm{C}_{22} \mathrm{H}_{35} \mathrm{OSi}^{+}(\mathrm{M}+\mathrm{H})$ 343.2457; found: 343.2443.

\section{Triisopropyl-[3-(5-bromo-2-methyl-2,3-dihydro-benzofuran-2-yl)-prop-1-ynyl]-silane (4e)}
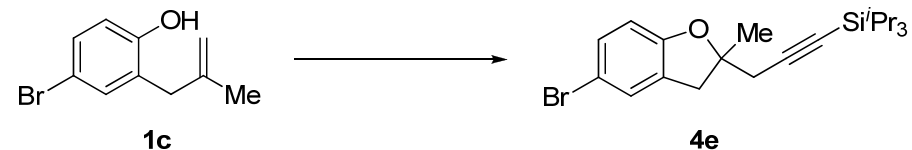

Column chromatography $\left(\mathrm{SiO}_{2}, \mathrm{PET} /\right.$ EtOAc 98:2) afforded product 4e $(0.11 \mathrm{~g}, 0.27 \mathrm{mmol}, 68 \%$ yield $)$ as a yellow oil. $\mathrm{R}_{\mathrm{f}} 0.80(\mathrm{PET} / \mathrm{EtOAc} 10: 1) .{ }^{1} \mathrm{H} \mathrm{NMR}\left(400 \mathrm{MHz}, \mathrm{CDCl}_{3}\right) \delta 7.25-7.23(\mathrm{~m}, 1 \mathrm{H}, \mathrm{Ar} H), 7.18(\mathrm{dd}, J=$ 
2.1, 8.4 Hz, 1H, ArH), $6.56(\mathrm{~d}, J=8.4 \mathrm{~Hz}, 1 \mathrm{H}, \operatorname{Ar} H), 3.37(\mathrm{~d}, J=16.2 \mathrm{~Hz}, 1 \mathrm{H}, \operatorname{ArCH}), 2.98(\mathrm{~d}, J=16.2 \mathrm{~Hz}, 1$ $\left.\mathrm{H}, \mathrm{ArCH}_{2}\right), 2.60\left(\mathrm{~m}, 2 \mathrm{H}, \mathrm{CH}_{2} \mathrm{C} \equiv \mathrm{C}\right), 1.57\left(\mathrm{~s}, 3 \mathrm{H}, \mathrm{CH}_{3}\right), 1.06-0.97$ (m, $\left.21 \mathrm{H}, \mathrm{TIPS}\right) .{ }^{13} \mathrm{C} \mathrm{NMR}(100 \mathrm{MHz}$, $\left.\mathrm{CDCl}_{3}\right) \delta 158.0,130.8,129.1,128.0,112.0,111.0,103.7,88.2,83.3,40.4,32.5,26.3,18.6,11.2$. IR $v 3730(\mathrm{w})$, 2942 (m), 2864 (m), 2360 (w), 2340 (w), 2176 (m), 1659 (w), 1605 (w), 1490 (m), 1575 (s), 1381 (m), 1294 (m), 1239 (s), 1178 (m), 1107 (m), 1072 (s), 1034 (s), 995 (m), 960 (m), 920 (m), 884 (s), 810 (s), 785 (m), 678 (s), 629 (s). HRMS (ESI) calcd for $\mathrm{C}_{21} \mathrm{H}_{32}{ }^{79} \mathrm{BrOSi}^{+}, \mathrm{C}_{21} \mathrm{H}_{32}{ }^{81} \mathrm{BrOSi}^{+}(\mathrm{M}+\mathrm{H})$ 407.1406, 409.1388; found: 407.1395, 409.1383.

2-Methyl-2-(3-(triisopropylsilyl)prop-2-ynyl)-2,3-dihydro-1-benzofuran-5-carbonitrile (4f)

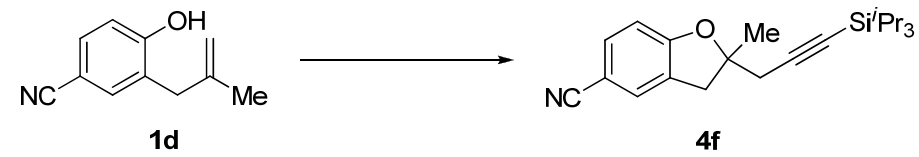

Column chromatography $\left(\mathrm{SiO}_{2}, \mathrm{PET} /\right.$ EtOAc 98:2) afforded product $\mathbf{4 f}$ (107 $\mathrm{mg}, 0.303 \mathrm{mmol}, 76 \%$ yield) as a yellow oil. $\mathrm{R}_{\mathrm{f}} 0.74$ (PET/EtOAc 5:1). ${ }^{1} \mathrm{H}$ NMR (400 MHz, $\left.\mathrm{CDCl}_{3}\right) \delta 7.48-7.40$ (m, $\left.2 \mathrm{H}, \mathrm{ArH}\right), 6.78(\mathrm{dd}, J=$ 9.0, 3.0 Hz, 1H, ArH), 3.45 (d, $\left.J=16.1 \mathrm{~Hz}, 1 \mathrm{H}, \mathrm{ArCH}_{2}\right), 3.04(\mathrm{~d}, J=16.1 \mathrm{~Hz}, 1 \mathrm{H}, \operatorname{ArCH}), 2.72(\mathrm{dm}, J=17.1$ $\left.\mathrm{Hz}, 1 \mathrm{H}, \mathrm{CH}_{2} \mathrm{C} \equiv \mathrm{C}\right), 2.62\left(\mathrm{dm}, J=17.1 \mathrm{~Hz}, 1 \mathrm{H}, \mathrm{CH}_{2} \mathrm{C} \equiv \mathrm{C}\right), 1.60\left(\mathrm{~m}, 3 \mathrm{H}, \mathrm{CH}_{3}\right), 1.15-0.88(\mathrm{~m}, 21 \mathrm{H}, \mathrm{TIPS}) .{ }^{13} \mathrm{C}$ NMR $\left(100 \mathrm{MHz}, \mathrm{CDCl}_{3}\right) \delta 162.5,133.6,129.1,128.4,124.0,119.7,110.3,103.0,89.3,83.7,39.8,32.7,26.5$, 18.5, 11.2. IR v 2943 (s), 2865 (s), 2349 (w), 2224 (m), 2177 (m), 1770 (w), 1614 (m), 1487 (s), 1464 (m), 1382 (w), 1300 (m), 1266 (m), 1247 (m), 1112 (w), 1108 (w), 1069 (m), 1035 (m), 996 (w), 923 (w), 882 (m), 821 (m), 788 (w), 739 (w), 677 (s). HRMS (ESI) calcd for $\mathrm{C}_{22} \mathrm{H}_{32} \mathrm{NOSi}^{+}$(M+H) 354.2253; found: 354.2250.

\section{Triisopropyl-[3-(5-nitro-2-methyl-2,3-dihydro-benzofuran-2-yl)-prop-1-ynyl]-silane (4g)}

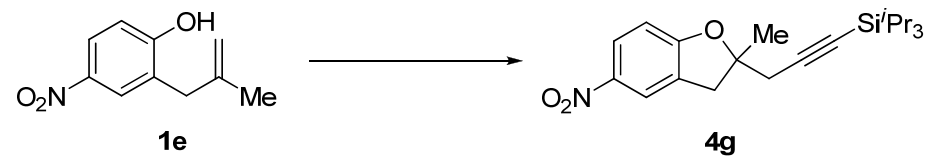

Column chromatography ( $\mathrm{SiO}_{2}, \mathrm{PET} /$ EtOAc 98:2) afforded product $\mathbf{4 g}$ (108 $\mathrm{mg}, 0.289 \mathrm{mmol}, 72 \%$ yield) as a yellow oil. $\mathrm{R}_{\mathrm{f}} 0.85$ (PET/EtOAc 5:1). ${ }^{1} \mathrm{H}$ NMR (400 MHz, $\left.\mathrm{CDCl}_{3}\right) \delta 8.12-8.03$ (m, $\left.2 \mathrm{H}, \mathrm{ArH}\right), 6.75(\mathrm{~m}, 1 \mathrm{H}$, $\operatorname{Ar} H), 3.47\left(\mathrm{~d}, J=16.6 \mathrm{~Hz}, 1 \mathrm{H}, \mathrm{ArCH} \mathrm{C}_{2}\right), 3.06\left(\mathrm{~d}, J=16.6 \mathrm{~Hz}, 1 \mathrm{H}, \mathrm{ArCH}_{2}\right), 2.75(\mathrm{~d}, J=17.1 \mathrm{~Hz}, 1 \mathrm{H}$, $\left.\mathrm{CH}_{2} \mathrm{C} \equiv \mathrm{C}\right), 2.65\left(\mathrm{~d}, \mathrm{~J}=17.1 \mathrm{~Hz}, 1 \mathrm{H}, \mathrm{CH}_{2} \mathrm{C} \equiv \mathrm{C}\right), 1.60\left(\mathrm{~s}, 3 \mathrm{H}, \mathrm{CH}_{3}\right), 1.02-0.94(\mathrm{~m}, 21 \mathrm{H}, \mathrm{TIPS}) .{ }^{13} \mathrm{C}$ NMR $(100$ $\left.\mathrm{MHz}, \mathrm{CDCl}_{3}\right) \delta 164.3,141.8,128.2,125.8,121.5,109.2,102.8,90.4,83.9,39.7,32.7,26.5,18.5,11.2$. IR $v$ 2942 (m), 2865 (m), 2349 (w), 2176 (w), 1621 (w), 1598 (m), 1523 (m), 1477 (m), 1339 (s), 1289 (m), 1272 (m), 1242 (m), $1120(\mathrm{w}), 1061$ (m), 1035 (m), 997 (w), 925 (m), 883 (m), 810 (w), 783 (w), 739 (w), 665 (m), 636 (m). HRMS (ESI) calcd for $\mathrm{C}_{21} \mathrm{H}_{32} \mathrm{NO}_{3} \mathrm{Si}^{+}(\mathrm{M}+\mathrm{H}) 374.2151$; found: 374.2133 .

\section{1-(2-Methyl-2-(3-(triisopropylsilyl)prop-2-ynyl)-2,3-dihydro-1-benzofuran-5-yl)ethanone (4h)}




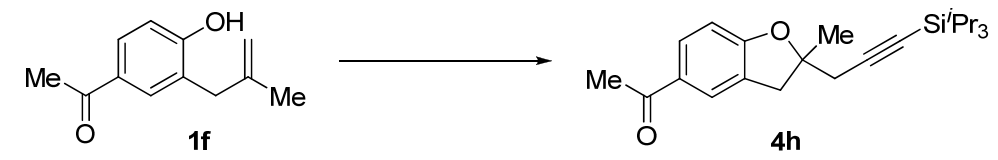

Column chromatography $\left(\mathrm{SiO}_{2}, \mathrm{PET} /\right.$ EtOAc 98:2) afforded product $4 \mathbf{h}$ (128 $\mathrm{mg}, 0.345 \mathrm{mmol}, 87 \%$ yield) as a yellow oil. Rf $0.54(\mathrm{PET} / \mathrm{EtOAc} 8: 1) .{ }^{1} \mathrm{H} \mathrm{NMR}\left(400 \mathrm{MHz}, \mathrm{CDCl}_{3}\right) \delta 7.78(\mathrm{~m}, 1 \mathrm{H}, \mathrm{ArH}), 7.75(\mathrm{~d}, J=1.9 \mathrm{~Hz}$, $1 \mathrm{H}, \operatorname{Ar} H), 6.72(\mathrm{~d}, J=8.2 \mathrm{~Hz}, 1 \mathrm{H}, \operatorname{Ar} H), 3.40(\mathrm{~d}, J=16.0 \mathrm{~Hz}, 1 \mathrm{H}, \operatorname{ArCH})_{2}, 3.01(\mathrm{~d}, J=16.0 \mathrm{~Hz}, 1 \mathrm{H}$, $\left.\mathrm{ArCH}_{2}\right), 2.70\left(\mathrm{~d}, \mathrm{~J}=16.8 \mathrm{~Hz}, 1 \mathrm{H}, \mathrm{CH}_{2} \mathrm{C} \equiv \mathrm{C}\right), 2.62\left(\mathrm{~d}, \mathrm{~J}=16.8 \mathrm{~Hz}, 1 \mathrm{H}, \mathrm{CH}_{2} \mathrm{C} \equiv \mathrm{C}\right), 2.51\left(\mathrm{~s}, 3 \mathrm{H}, \mathrm{COCH}_{3}\right), 1.58$ $\left(\mathrm{s}, 3 \mathrm{H}, \mathrm{CH}_{3}\right), 1.09-0.85$ (m, $\left.21 \mathrm{H}, \mathrm{TIPS}\right) .{ }^{13} \mathrm{C} \mathrm{NMR}\left(100 \mathrm{MHz}, \mathrm{CDCl}_{3}\right) \delta 196.6,163.1,130.6,130.4,127.4$, 125.8, 109.1, 103.5, 89.2, 83.4, 39.9, 32.6, 26.4, 18.5, 11.2 (one carbon cannot be resolved). IR v 2942 (s), 2864 (s), $2176(\mathrm{~m}), 1676(\mathrm{~s}), 1609$ (s), 1589 (m), 1489 (m), $1464(\mathrm{~m}), 1438(\mathrm{~m}), 1381$ (w), $1358(\mathrm{~m}), 1305$ (w), 1265 (s), $1170(\mathrm{w}), 1110(\mathrm{w}), 1075(\mathrm{~m}), 1056$ (m), $1034(\mathrm{~m}), 996(\mathrm{w}), 956(\mathrm{w}), 911(\mathrm{~m}), 883$ (s), $816(\mathrm{~m}), 787$ (w), 729 (w), 675 (s), 634 (s), 612 (m). HRMS (ESI) expected for $\mathrm{C}_{23} \mathrm{H}_{34} \mathrm{O}_{2} \mathrm{Si}^{+}(\mathrm{M}+\mathrm{H})$ 371.2406; found: 371.2419.

Triisopropyl-[3-(6-bromo-2-methyl-2,3-dihydro-benzofuran-2-yl)-prop-1-ynyl]-silane (4i)

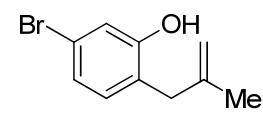

$1 \mathrm{~g}$

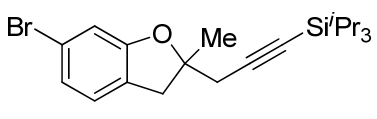

4i

Column chromatography $\left(\mathrm{SiO}_{2}, \mathrm{PET} / \mathrm{EtOAc} 98: 2\right)$ afforded product $4 \mathbf{i}$ (107 $\mathrm{mg}, 0.263 \mathrm{mmol}, 66 \%$ yield) as a yellow oil. $\mathrm{R}_{\mathrm{f}} 0.86(\mathrm{PET} / \mathrm{EtOAc} 10: 1) .{ }^{1} \mathrm{H} \mathrm{NMR}\left(400 \mathrm{MHz}, \mathrm{CDCl}_{3}\right) \delta 6.98-6.94(\mathrm{~m}, 2 \mathrm{H}, \mathrm{ArH}), 6.65(\mathrm{~m}, 1 \mathrm{H}$, $\mathrm{Ar} H), 3.41\left(\mathrm{~d}, J=16.3 \mathrm{~Hz}, 1 \mathrm{H}, \mathrm{ArCH}_{2}\right), 2.99$ (d, $\left.J=\mathrm{Hz}, 1 \mathrm{H}, \mathrm{ArCH}\right)_{2}, 2.72\left(\mathrm{~d}, J=16.7 \mathrm{~Hz}, 1 \mathrm{H}, \mathrm{CH}_{2} \mathrm{C} \equiv \mathrm{C}\right)$, $2.62\left(\mathrm{~d}, J=16.7 \mathrm{~Hz}, 1 \mathrm{H}, \mathrm{CH}_{2} \mathrm{C} \equiv \mathrm{C}\right), 1.58\left(\mathrm{~s}, 3 \mathrm{H}, \mathrm{CH}_{3}\right), 1.07-0.95$ (m, $\left.21 \mathrm{H}, \mathrm{TIPS}\right) .{ }^{13} \mathrm{C} \mathrm{NMR}(100 \mathrm{MHz}$, $\left.\mathrm{CDCl}_{3}\right) \delta 159.2,129.5,128.0,123.2,119.3,108.3,103.6,87.3,83.4,42.0,32.8,26.6,18.6,11.2$. IR $v 3743(\mathrm{w})$, 2941 (m), 2865 (m), 2176 (m), 1605 (m), 1584 (m), 1449 (s), 1382 (w), 1319 (w), 1266 (s), 1241 (s), 1171 (w), $1111(\mathrm{w}), 1074$ (m), 995 (m), 936 (w), 906 (s), 882 (s), 825 (w), 765 (s), 707 (m), 664 (s). HRMS (ESI) calcd for $\mathrm{C}_{21} \mathrm{H}_{32}{ }^{79} \mathrm{BrOSi}^{+}, \mathrm{C}_{21} \mathrm{H}_{32}{ }^{81} \mathrm{BrOSi}^{+}(\mathrm{M}+\mathrm{H})$ 407.1406, 409.1388; found: 407.1391, 409.1367.

Triisopropyl-[3-(7-bromo-2-methyl-2,3-dihydro-benzofuran-2-yl)-prop-1-ynyl]-silane (4j)

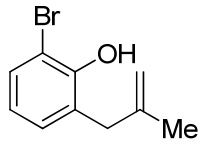

$1 \mathrm{~h}$

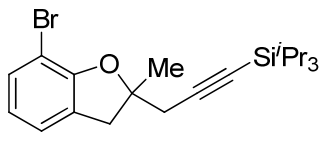

4j

Column chromatography $\left(\mathrm{SiO}_{2}, \mathrm{PET} /\right.$ EtOAc 98:2) afforded product 4j (84 mg, $0.21 \mathrm{mmol}, 52 \%$ yield) as a yellow oil. $\mathrm{R}_{\mathrm{f}} 0.78$ (PET/EtOAc 10:1). ${ }^{1} \mathrm{H}$ NMR (400 MHz, $\left.\mathrm{CDCl}_{3}\right) \delta$ 7.23-7.27 (m, $\left.1 \mathrm{H}, \mathrm{ArH}\right), 7.06(\mathrm{dd}, J=$ 7.4, 1.2 Hz, 1H, ArH), 6.70 (t, $J=7.6 \mathrm{~Hz}, 1 \mathrm{H}, \mathrm{ArH}), 3.48$ (d, J=16.0 Hz, $1 \mathrm{H}, \mathrm{ArCH}$ ), 3.08 (d, J=16.0 Hz, 1 $\left.\mathrm{H}, \mathrm{ArCH}_{2}\right), 2.71\left(\mathrm{~m}, 2 \mathrm{H}, \mathrm{CH}_{2} \mathrm{C} \equiv \mathrm{C}\right), 1.62\left(\mathrm{~s}, 3 \mathrm{H}, \mathrm{CH}_{3}\right), 0,99-1.05$ (m, $\left.21 \mathrm{H}, \mathrm{TIPS}\right) .{ }^{13} \mathrm{C} \mathrm{NMR}(100 \mathrm{MHz}$, $\left.\mathrm{CDCl}_{3}\right) \delta 156.2,128.0,131.1,124.0,121.5,103.7,102.7,88.2,83.2,41.4,32.6,26.3,18.6,11.2 . \mathrm{IR} v 2941(\mathrm{~m})$, 
$2864(\mathrm{~m}), 2176(\mathrm{w}), 1648$ (w), 1604 (w), 1584 (w), 1455 (s), $1382(\mathrm{w}), 1329$ (w), $1291(\mathrm{w}), 1246$ (w), 1216 (m), $1112(\mathrm{w}), 1072$ (m), 1034 (m), 1028 (m), 994 (w), 920 (w), 882 (s), 783 (w), 759 (s), 714 (m), 664 (s), 644 (s). HRMS (ESI) calcd for $\mathrm{C}_{21} \mathrm{H}_{32}{ }^{79} \mathrm{BrOSi}^{+}, \mathrm{C}_{21} \mathrm{H}_{32}{ }^{81} \mathrm{BrOSi}^{+}$(M+H) 407.1406, 409.1388; found: 407.1409, 409.1393.

\section{(3-(2,3-Dihydro-benzofuran-2-yl)-prop-1-ynyl)triisopropyl silane (4k)}

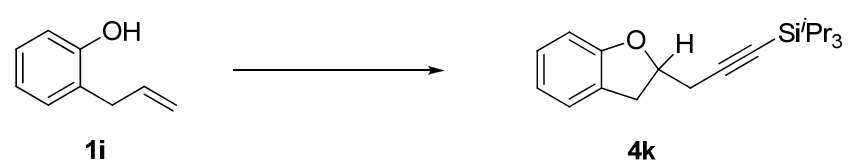

Column chromatography ( $\mathrm{SiO}_{2}$, PET/DCM 95:5) afforded product 4k (47 mg, $0.15 \mathrm{mmol}, 37 \%$ yield) as a yellow oil. $\mathrm{R}_{\mathrm{f}} 0.88\left(\mathrm{PET} /\right.$ EtOAc 10:1). ${ }^{1} \mathrm{H}$ NMR (400 MHz, $\left.\mathrm{CDCl}_{3}\right) \delta 7.17(\mathrm{~d}, J=7.2 \mathrm{~Hz}, 1 \mathrm{H}, \mathrm{ArH}), 7.10(\mathrm{t}, J$ $=8.1 \mathrm{~Hz}, 1 \mathrm{H}, \operatorname{Ar} H), 6.83(\mathrm{t}, J=7.4 \mathrm{~Hz}, 1 \mathrm{H}, \operatorname{Ar} H), 6.75(\mathrm{~d}, J=8.1 \mathrm{~Hz}, 1 \mathrm{H}, \operatorname{Ar} H), 4.92(\mathrm{~m}, 1 \mathrm{H}, C H), 3.36(\mathrm{dd}$, $J=15.8,9.1 \mathrm{~Hz}, 1 \mathrm{H}, \mathrm{ArCH}), 3.19\left(\mathrm{dd}, J=15.6,6.5 \mathrm{~Hz}, 1 \mathrm{H}, \mathrm{ArCH}_{2}\right), 2.76(\mathrm{dd}, J=16.7,4.5 \mathrm{~Hz}, 1 \mathrm{H}$, $\left.\mathrm{CH}_{2} \mathrm{C} \equiv \mathrm{C}\right), 2.67\left(\mathrm{dd}, J=16.7,7.7 \mathrm{~Hz}, 1 \mathrm{H}, \mathrm{CH}_{2} \mathrm{C} \equiv \mathrm{C}\right), 1.09-0.97$ (m, $\left.21 \mathrm{H}, \mathrm{TIPS}\right) .{ }^{13} \mathrm{C} \mathrm{NMR}(100 \mathrm{MHz}$ $\left.\mathrm{CDCl}_{3}\right) \delta 159.4,128.0,126.3,125.0,120.5,109.3,103.3,83.2,80.5,34.6,27.0,18.6,11.2$. IR v $2942(\mathrm{~s}), 2864$ (s), 2176 (m), 1599 (m), 1481 (s), 1463 (s), 1383 (w), 1365 (w), $1336(\mathrm{w}), 1323$ (w), 1294 (w), 1230 (s), 1172 (w), $1142(\mathrm{w}), 1098(\mathrm{w}), 1074(\mathrm{w}), 1026(\mathrm{~m}), 1017(\mathrm{~m}), 984(\mathrm{~m}), 920(\mathrm{w}), 884(\mathrm{~s}), 858(\mathrm{~m}), 817(\mathrm{w}), 794(\mathrm{w})$, $748(\mathrm{~s}), 710(\mathrm{~m}), 677$ (s), 660 (s), 638 (s), 615(m). HRMS (ESI) calcd for $\mathrm{C}_{20} \mathrm{H}_{31} \mathrm{OSi}^{+}$(M+H) 315.2144; found: 315.2139 .

\section{Triisopropyl-(3-(2-methylchroman-2-yl)-prop-1-ynyl)-silane (4l)}
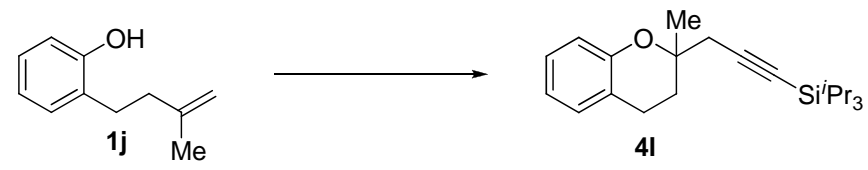

Column chromatography ( $\mathrm{SiO}_{2}, \mathrm{PET} / \mathrm{DCM}$ 95:5) afforded product $4 \mathbf{l}$ (63 mg, $0.18 \mathrm{mmol}, 46 \%$ yield) as a colorless oil. $\mathrm{R}_{\mathrm{f}} 0.94$ (PET/EtOAc 10:1). ${ }^{1} \mathrm{H}$ NMR (400 MHz, $\left.\mathrm{CDCl}_{3}\right) \delta 7.13-7.05$ (m, $\left.2 \mathrm{H}, \mathrm{Ar} H\right), 6.84(\mathrm{td}, J=$ 1.0, 7.4 Hz, $1 \mathrm{H}, \operatorname{Ar} H), 6.79(\mathrm{~d}, J=8.1 \mathrm{~Hz}, 1 \mathrm{H}, \operatorname{ArH}), 2.80\left(\mathrm{~m}, 2 \mathrm{H}, \operatorname{ArCH}_{2}\right), 2.62(\mathrm{~d}, J=16.7 \mathrm{~Hz}, 1 \mathrm{H}$, $\left.\mathrm{CH}_{2} \mathrm{C} \equiv \mathrm{C}\right), 2.53\left(\mathrm{~d}, \mathrm{~J}=16.7 \mathrm{~Hz}, 1 \mathrm{H}, \mathrm{CH}_{2} \mathrm{C} \equiv \mathrm{C}\right), 2.14\left(\mathrm{~m}, 1 \mathrm{H}, \mathrm{CH}_{2}\right), 1.88\left(\mathrm{~m}, 1 \mathrm{H}, \mathrm{CH}_{2}\right), 1.48\left(\mathrm{~s}, 3 \mathrm{H}, \mathrm{CH}_{3}\right)$, 1.16 - 1.03 (m, $21 \mathrm{H}$, TIPS). ${ }^{13} \mathrm{C}$ NMR (100 MHz, $\left.\mathrm{CDCl}_{3}\right) \delta 153.5,129.5,127.4,121.1,120.0,117.3,104.5$, 83.1, 75.8, 30.6, 29.9, 25.1, 22.1, 18.7, 11.3. IR v 2941 (s), 2864 (s), $2174(\mathrm{~m}), 1612(\mathrm{w}), 1583$ (m), 1489 (s), 1456 (s), 1379 (m), 1355 (w), 1306 (m), 1272 (w), 1270 (w), 1242 (s), 1216 (m), 1190 (w), 1148 (m), 1133 (m), 1111 (s), 1088 (s), 1037 (s), 995 (m), 948 (m), 928 (m), 883 (s), $830(\mathrm{w}), 753$ (s), 728 (w), 710 (w), 677 (s), 667 (s), 637 (s). HRMS (ESI) calcd for $\mathrm{C}_{22} \mathrm{H}_{35} \mathrm{OSi}^{+}(\mathrm{M}+\mathrm{H})$ 343.2457; found: 343.2456.

\section{3-Methyl-3-(3-(triisopropylsilyl)prop-2-ynyl)isobenzofuran-1(3H)-one (6a)}




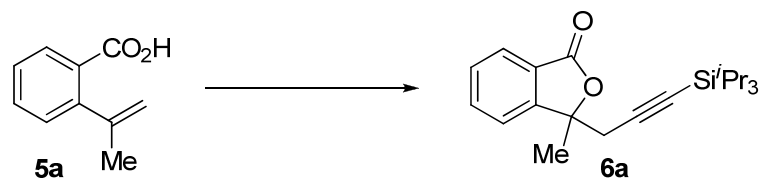

Column chromatography $\left(\mathrm{SiO}_{2}, \mathrm{PET} /\right.$ EtOAc 98:2) afforded product 6a (70 mg, $0.33 \mathrm{mmol}, 83 \%$ yield) as a colorless solid. $\mathrm{R}_{\mathrm{f}} 0.73$ (PET/EtOAc 5:1). Mp $104-105{ }^{\circ} \mathrm{C} .{ }^{1} \mathrm{H}$ NMR (400 MHz, $\left.\mathrm{CDCl}_{3}\right) \delta 7.85$ (d, $J=7.6 \mathrm{~Hz}$, $1 \mathrm{H}, \operatorname{Ar} H), 7.64(\mathrm{td}, J=7.7,1.0 \mathrm{~Hz}, 1 \mathrm{H}, \operatorname{Ar} H), 7.59(\mathrm{~d}, J=7.6 \mathrm{~Hz}, 1 \mathrm{H}, \operatorname{Ar} H), 7.51(\mathrm{td}, J=7.6,1.2 \mathrm{~Hz}, 1 \mathrm{H}$, $\mathrm{ArH}), 2.97\left(\mathrm{~d}, J=16.8 \mathrm{~Hz}, 1 \mathrm{H}, \mathrm{CH}_{2} \mathrm{C} \equiv \mathrm{C}\right), 2.83\left(\mathrm{~d}, J=16.8 \mathrm{~Hz}, 1 \mathrm{H}, \mathrm{CH}_{2} \mathrm{C} \equiv \mathrm{C}\right), 1.74\left(\mathrm{~s}, 3 \mathrm{H}, \mathrm{CH}_{3}\right), 1.05-0.88$ (m, $21 \mathrm{H}, \mathrm{TIPS}) .{ }^{13} \mathrm{C}$ NMR $\left(100 \mathrm{MHz}, \mathrm{CDCl}_{3}\right) \delta 169.4,152.7,133.9,129.3,126.0,125.7,121.5,101.6,85.2$, 84.8, 32.3, 25.1, 19.0, 10.6. IR v 2942 (s), 2864 (s), 2174 (m), 1767 (s), 1615 (w), 1465 (m), 1382 (w), 1363 (w), $1337(\mathrm{w}), 1312(\mathrm{w}), 1286(\mathrm{~m}), 1248(\mathrm{w}), 1222(\mathrm{w}), 1164(\mathrm{w}), 1121(\mathrm{~m}), 1077(\mathrm{w}), 1035(\mathrm{~s}), 1001(\mathrm{~m}), 916$ (m), $884(\mathrm{~m}), 805$ (m), 765 (w), 726 (w), 696 (s), 674 (s), 618 (s). HRMS (ESI) calcd for $\mathrm{C}_{21} \mathrm{H}_{31} \mathrm{O}_{2} \mathrm{Si}^{+}(\mathrm{M}+\mathrm{H})$ 343.2093; found: 343.2080 .

5-Methoxy-3-methyl-3-(3-(triisopropylsilyl)prop-2-ynyl)isobenzofuran-1(3H)-one (6b)

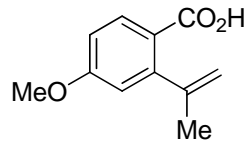

$5 b$

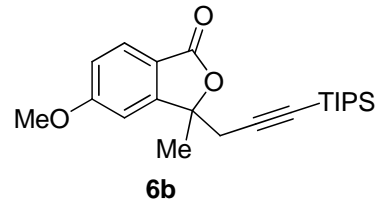

$6 b$

Column chromatography $\left(\mathrm{SiO}_{2}\right.$, Hexane/EtOAc 95:5) afforded product $6 \mathbf{b}$ (120 mg, $0.323 \mathrm{mmol}, 80 \%$ yield) as a yellow solid. $\mathrm{R}_{\mathrm{f}} 0.4$ (PET/EtOAc 5:1). Mp $73-76^{\circ} \mathrm{C} .{ }^{1} \mathrm{H}$ NMR $\left(400 \mathrm{MHz}, \mathrm{CDCl}_{3}\right) \delta 7.76(\mathrm{~m}, 1 \mathrm{H}, \mathrm{ArH})$, 7.07-7.00 (m, $2 \mathrm{H}, \mathrm{ArH}), 3.91\left(\mathrm{~m}, 3 \mathrm{H}, \mathrm{OCH}_{3}\right), 2.99\left(\mathrm{~d}, J=16.9 \mathrm{~Hz}, 1 \mathrm{H}, \mathrm{CH}_{2} \mathrm{C} \equiv \mathrm{C}\right), 2.83(\mathrm{~d}, J=16.9 \mathrm{~Hz}, 1 \mathrm{H}$, $\left.\mathrm{CH}_{2} \mathrm{C} \equiv \mathrm{C}\right), 1.76\left(\mathrm{~s}, 3 \mathrm{H}, \mathrm{CH}_{3}\right), 1.10-0.95$ (m, $\left.21 \mathrm{H}, \mathrm{TIPS}\right) .{ }^{13} \mathrm{C}$ NMR $\left(100 \mathrm{MHz}, \mathrm{CDCl}_{3}\right) \delta 164.7,155.5,127.2$, 118.2, 116.3, 105.8, 101.8, 84.6, 84.3, 55.8, 32.1, 25.1, 18.5, 11.1 (one aromatic carbon cannot be resolved). IR v $2968(\mathrm{~m}), 2943(\mathrm{w}), 2893(\mathrm{~m}), 2865(\mathrm{w}), 2178(\mathrm{~s}), 1760$ (s), 1608 (m), $1492(\mathrm{~m}), 1464(\mathrm{~m}), 1382$ (m), 1341 (m), 1330 (m), 1295 (m), 1247 (s), 1203 (w), 1180 (w), 1119 (m), 1069 (m), 1037 (s), 996 (w), 948 (w), 918 (w), $884(\mathrm{~m}), 842(\mathrm{w}), 787(\mathrm{w}), 738(\mathrm{w}), 699(\mathrm{~m}), 677(\mathrm{~m}), 626(\mathrm{~m})$. HRMS (ESI) calcd for $\mathrm{C}_{22} \mathrm{H}_{33} \mathrm{O}_{3} \mathrm{Si}^{+}(\mathrm{M}+\mathrm{H})$ 373.2199; found: 373.2204 .

\section{3-Phenyl-3-(3-(triisopropylsilyl)prop-2-ynyl)isobenzofuran-1(3H)-one (6c)}

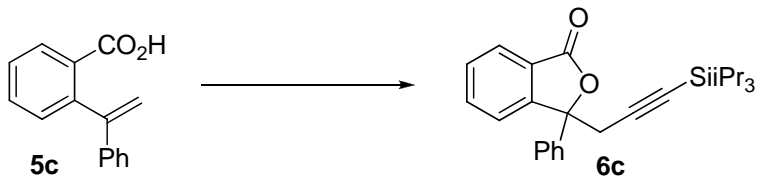

Column chromatography $\left(\mathrm{SiO}_{2}, \mathrm{PET} /\right.$ EtOAc 98:2) afforded product 6c (113 mg, $0.279 \mathrm{mmol}, 70 \%$ yield) as a colorless solid. $\mathrm{R}_{\mathrm{f}} 0.67$ (PET/EtOAc 5:1). Mp $58-60{ }^{\circ} \mathrm{C} .{ }^{1} \mathrm{H}$ NMR $\left(400 \mathrm{MHz}, \mathrm{CDCl}_{3}\right) \delta 7.90(\mathrm{~d}, J=7.6 \mathrm{~Hz}$, $1 \mathrm{H}, \operatorname{Ar} H), 7.70-7.60(\mathrm{~m}, 2 \mathrm{H}, \operatorname{Ar} H), 7.57-7.49$ (m, $3 \mathrm{H}, \mathrm{ArH}, \mathrm{Ph} H), 7.42-7.27$ (m, $3 \mathrm{H}, \mathrm{Ph} H), 3.41(\mathrm{~d}, J=$ 
$\left.16.8 \mathrm{~Hz}, 1 \mathrm{H}, \mathrm{CH}_{2} \mathrm{C} \equiv \mathrm{C}\right), 3.28\left(\mathrm{~d}, J=16.8 \mathrm{~Hz}, 1 \mathrm{H}, \mathrm{CH}_{2} \mathrm{C} \equiv \mathrm{C}\right), 0.97-0.80(\mathrm{~m}, 21 \mathrm{H}, \mathrm{TIPS}) .{ }^{13} \mathrm{C}$ NMR (100 $\left.\mathrm{MHz}, \mathrm{CDCl}_{3}\right) \delta 169.4,151.3,139.0,134.1,129.5,128.7,128.7,126.4,125.8,125.5,122.9,101.1,87.6,85.2$, 32.6, 18.4 11.1. IR $v 3062(\mathrm{w}), 2942$ (m), 2864 (m), $2174(\mathrm{w}), 1772$ (s), 1603 (w), 1496 (w), 1465 (m), 1419 (w), 1386 (w), 1366 (w), 1339 (w), 1286 (m), 1228 (m), 1218 (m), 1189 (w), 1159 (w), 1094 (s), 1019 (m), 991 (s), 942 (m), 918 (w), 884 (s), 766 (s), 723 (w), 696 (s), 678 (s), 618 (m). HRMS (ESI) calcd for $\mathrm{C}_{26} \mathrm{H}_{33} \mathrm{O}_{2} \mathrm{Si}^{+}$ $(\mathrm{M}+\mathrm{H})$ 405.2250; found: 405.2259 .

\section{3-Methyl-3-(4-(triisopropylsilyl)but-3-ynyl)benzofuran-1(3H)-one (6d)}
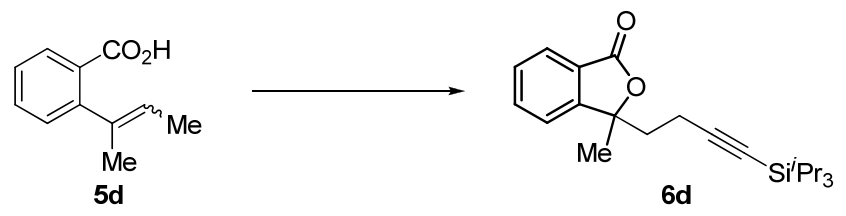

Column chromatography $\left(\mathrm{SiO}_{2}, \mathrm{PET} /\right.$ EtOAc 98:2) afforded product 6d (80 mg, $0.22 \mathrm{mmol}, 56 \%$ yield) as a yellow oil. $\mathrm{R}_{\mathrm{f}} 0.75$ (PET/EtOAc 5:1). ${ }^{1} \mathrm{H}$ NMR (400 MHz, $\left.\mathrm{CDCl}_{3}\right) \delta 7.86(\mathrm{dd}, J=8.0,1.0 \mathrm{~Hz}, 1 \mathrm{H}, \mathrm{ArH}), 7.67$ $(\mathrm{td}, J=7.5,1.0 \mathrm{~Hz}, 1 \mathrm{H}, \operatorname{Ar} H), 7.51(\mathrm{t}, J=7.5,1 \mathrm{H}, \operatorname{Ar} H), 7.40(\mathrm{dd}, J=0.5,7.5 \mathrm{~Hz}, 1 \mathrm{H}, \operatorname{Ar} H), 2.31(\mathrm{~m}, 2 \mathrm{H}$, $\left.\mathrm{CH}_{2} \mathrm{CH}_{2} \mathrm{C} \equiv \mathrm{C}\right), 2.12\left(\mathrm{~m}, 1 \mathrm{H}, \mathrm{CH}_{2} \mathrm{CH}_{2} \mathrm{C} \equiv \mathrm{C}\right), 1.96\left(\mathrm{~m}, 1 \mathrm{H}, \mathrm{CH}_{2} \mathrm{CH}_{2} \mathrm{C} \equiv \mathrm{C}\right), 1.66\left(\mathrm{~s}, 3 \mathrm{H}, \mathrm{CH}_{3}\right), 1.11-0.91(\mathrm{~m}, 21$ $\mathrm{H}, \mathrm{TIPS}) .{ }^{13} \mathrm{C} \mathrm{NMR}\left(100 \mathrm{MHz}, \mathrm{CDCl}_{3}\right) \delta 169.6,153.0,134.3,129.2,125.9,125.8,121.0,107.0,86.7,81.0$, 39.2, 25.8, 18.6, 14.9, 11.2. IR v 2942 (m), $2865(\mathrm{~m}), 2174(\mathrm{w}), 1767(\mathrm{~s}), 1615$ (w), 1465 (m), 1382 (w), 1363 (w), $1312(\mathrm{w}), 1286(\mathrm{~m}), 1248$ (w), 1222 (w), 1164 (m), 1121 (w), 1035 (s), 1001 (m), 916 (m), 884 (m), 805 (w), $726(\mathrm{w}), 696(\mathrm{~m}), 674(\mathrm{~s}), 618(\mathrm{~m})$. HRMS (ESI) expected for $\mathrm{C}_{22} \mathrm{H}_{33} \mathrm{O}_{2} \mathrm{Si}^{+}(\mathrm{M}+\mathrm{H})$ 357.2250; found: 357.2262 .

\section{3-Methyl-3-(3-(triisopropylsilyl)prop-2-ynyl)isochroman-1-one (6e)}

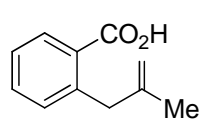

$5 e$

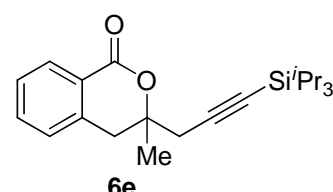

$6 e$

Column chromatography $\left(\mathrm{SiO}_{2}, \mathrm{PET} /\right.$ EtOAc 98:2) afforded product 6e (115 mg, $0.322 \mathrm{mmol}, 80 \%$ yield) as a colorless solid. $\mathrm{R}_{\mathrm{f}} 0.72$ (PET/EtOAc 5:1). Mp $71-73{ }^{\circ} \mathrm{C} .{ }^{1} \mathrm{H}$ NMR $\left(400 \mathrm{MHz}, \mathrm{CDCl}_{3}\right) \delta 8.07(\mathrm{dd}, J=7.7,1.0$ $\mathrm{Hz}, 1 \mathrm{H}, \mathrm{Ar} H), 7.54(\mathrm{td}, J=7.4,1.4 \mathrm{~Hz}, 1 \mathrm{H}, \mathrm{Ar} H), 7.38$ (dt, $J=7.4,0.5, \mathrm{~Hz}, 1 \mathrm{H}, \mathrm{ArH}), 7.22(\mathrm{~d}, J=7.4 \mathrm{~Hz}, 1$ $\mathrm{H}, \mathrm{ArH}), 3.33\left(\mathrm{~d}, J=16.3 \mathrm{~Hz}, 1 \mathrm{H}, \mathrm{ArCH}_{2}\right), 3.07$ (d, $\left.J=16.3 \mathrm{~Hz}, 1 \mathrm{H}, \mathrm{ArCH}_{2}\right), 2.68\left(\mathrm{~m}, 2 \mathrm{H}, \mathrm{CH}_{2} \mathrm{C} \equiv \mathrm{C}\right), 1.56$ (s, $\left.3 \mathrm{H}, \mathrm{CH}_{3}\right), 1.08-1.03$ (m, $\left.21 \mathrm{H}, \mathrm{TIPS}\right) .{ }^{13} \mathrm{C} \mathrm{NMR}\left(100 \mathrm{MHz}, \mathrm{CDCl}_{3}\right) \delta 164.4,137.5,134.0,130.1,128.1$, 127.7, 124.6, 102.9, 84.5, 81.7, 36.5, 31.8, 25.3, 18.6, 11.2. IR v 3330 (m), 3162 (m), 3067 (m), 3034 (m), 2943 (s), 2859 (s), 2177 (m), 2114 (m), 1727 (s), 1669 (m), 1649 (m), 1599 (m), 1550 (s), 1498 (m), 1463 (s), 1441 (m), 1385 (m), 1350 (m), 1314 (s), 1280 (s), 1226 (s), 1175 (m), 1114 (s), 1070 (s), 1034 (s), 885 (m), 845 (w), 798 (w), 746 (s), 677 (s); HRMS (ESI) calcd for $\mathrm{C}_{22} \mathrm{H}_{33} \mathrm{O}_{2} \mathrm{Si}^{+}$(M+H) 357.2250; found: 356.2265. 
2-(Benzyloxy)-3-methyl-3-(3-(triisopropylsilyl)prop-2-ynyl)isoindolin-1-one (8a) and 3-methyl-3-(3(triisopropylsilyl)prop-2-ynyl)isobenzofuran-1(3H)-one $O$-benzyl oxime (8b)
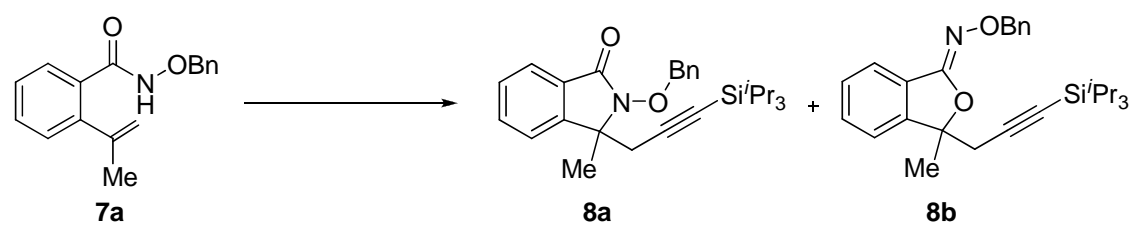

Column chromatography $\left(\mathrm{SiO}_{2}\right.$, PET/EtOAc 19:1) afforded product 8a (73 mg, $0.16 \mathrm{mmol}, 41 \%$ yield) as a yellow solid and $\mathbf{8 b}$ (49 mg, $0.11 \mathrm{mmol}, 28 \%$ yield) as a yellow solid. Product 8a: $\mathrm{R}_{\mathrm{f}} 0.57$ (PET/EtOAc 5:1). Mp 66-70 ${ }^{\circ} \mathrm{C} .{ }^{1} \mathrm{H}$ NMR $\left(400 \mathrm{MHz}, \mathrm{CDCl}_{3}\right) \delta 7.86(\mathrm{~d}, J=7.6 \mathrm{~Hz} ; \mathrm{ArH}), 7.61-7.52(\mathrm{~m}, 4 \mathrm{H}, \mathrm{Ph} H), 7.71-7.35$ (m, $4 \mathrm{H}, \mathrm{ArH}, \mathrm{Ph} H), 5.38$ (d, $\left.J=10.1 \mathrm{~Hz}, 1 \mathrm{H}, \mathrm{CH}_{2} \mathrm{OPh}\right), 5.28\left(\mathrm{~d}, J=10.1 \mathrm{~Hz}, 1 \mathrm{H}, \mathrm{CH}_{2} \mathrm{OPh}\right), 2.97(\mathrm{~d}, J=17.0$ $\left.\mathrm{Hz}, 1 \mathrm{H}, \mathrm{CH}_{2} \mathrm{C} \equiv \mathrm{C}\right), 2.71\left(\mathrm{~d}, \mathrm{~J}=17.0 \mathrm{~Hz}, 1 \mathrm{H}, \mathrm{CH}_{2} \mathrm{C} \equiv \mathrm{C}\right), 1.54\left(\mathrm{~s}, 3 \mathrm{H}, \mathrm{CH}_{3}\right), 1.05-0.84(\mathrm{~m}, 21 \mathrm{H}, \mathrm{TIPS}) .{ }^{13} \mathrm{C}$ NMR (100 MHz, $\left.\mathrm{CDCl}_{3}\right) \delta 164.6,146.4,135.3,132.0,129.4,129.1,128.7,128.5,128.3,123.6,121.4,102.5$, 84.4, 79.0, 65.0, 29.5, 22.8, 18.4, 11.0. IR v 3317 (broad, w), 3066 (w), 3034 (w), 2942 (s), 2892 (m), 2865 (s), 2249 (w), 2177 (m), 1713 (s), 1619 (w), 1464 (s), 1422 (w), 1375 (m), 1353 (w), 1319 (w), 1242 (w), 1219 (w), $1195(\mathrm{w}), 1161(\mathrm{w}), 1141(\mathrm{w}), 1141(\mathrm{w}), 1098(\mathrm{w}), 1065$ (m), 1032 (m), 992 (m), 969 (m), 942 (m), 913 (s), 884 (s), 839 (w), 791 (w), 763 (m), 735 (s), 693 (s), 670 (s). HRMS (ESI) expected for $\mathrm{C}_{28} \mathrm{H}_{38} \mathrm{NO}_{2} \mathrm{Si}^{+}(\mathrm{M}+\mathrm{H})$ 448.2672; found: 448.2678. Product 8b: $\mathrm{R}_{\mathrm{f}} 0.73$ (PET/EtOAc 5:1). Mp $63-66{ }^{\circ} \mathrm{C} .{ }^{1} \mathrm{H} \mathrm{NMR}\left(400 \mathrm{MHz} \mathrm{CDCl}_{3}\right)$ $\delta 7.62(\mathrm{~d}, J=7.6 \mathrm{~Hz} ; \mathrm{ArH}), 7.52-7.25(\mathrm{~m}, 8 \mathrm{H}, \mathrm{ArH}, \mathrm{PhH}), 5.13\left(\mathrm{~s}, 2 \mathrm{H}, \mathrm{CH}_{2} \mathrm{OPh}\right), 2.97(\mathrm{~d}, J=16.7 \mathrm{~Hz}, 1 \mathrm{H}$, $\left.\mathrm{CH}_{2} \mathrm{C} \equiv \mathrm{C}\right), 2.82\left(\mathrm{~d}, \mathrm{~J}=16.7 \mathrm{~Hz}, 1 \mathrm{H}, \mathrm{CH}_{2} \mathrm{C} \equiv \mathrm{C}\right), 1.74\left(\mathrm{~s}, 3 \mathrm{H}, \mathrm{CH}_{3}\right), 1.05-0.93(\mathrm{~m}, 21 \mathrm{H}, \mathrm{TIPS}) .{ }^{13} \mathrm{C}$ NMR $(100$ $\left.\mathrm{MHz}_{,} \mathrm{CDCl}_{3}\right) \delta 155.1,147.0,138.1,130.5,128.8,128.5,128.4,128.2,127.6,121.7,121.3,102.3,90.0,84.1$, 76.6, 32.9, 25.7, 18.5, 11.2. IR v 3166 (w), 3065 (w), 3032 (s), 2941 (m), 2893 (s), 2865 (m), 2178 (sh), 1721 (sh), 1688 (s), 1662 (sh), 1493 (sh), 1466 (s), 1415 (sh), 1355 (m), 1304 (m), 1249 (w), 1209 (w), 1160 (w), 1122 (w), 1076 (sh), 1040 (s), 994 (sh), 964 (m), 914 (s), 884 (s), 838 (w), 807 (w), 756 (sh), 755 (s), 736 (s), 700 (s), 673 (s), 604 (m). HRMS (ESI) expected for $\mathrm{C}_{28} \mathrm{H}_{38} \mathrm{NO}_{2} \mathrm{Si}^{+}(\mathrm{M}+\mathrm{H})$ 448.2672; found: 448.2660 .

\section{3,3,5-Trimethyl-5-(3-(triisopropylsilyl)prop-2-ynyl)dihydrofuran-2(3H)-one (10a)}

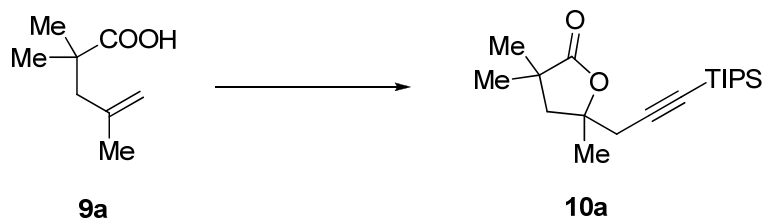

Column chromatography ( $\mathrm{SiO}_{2}$, PET/EtOAc 99:1) afforded product 10a (91 mg, $0.28 \mathrm{mmol}, 70 \%$ yield) as a yellow oil. $\mathrm{R}_{\mathrm{f}} 0.69$ (PET/EtOAc 5: 1). ${ }^{1} \mathrm{H} \mathrm{NMR}\left(400 \mathrm{MHz}, \mathrm{CDCl}_{3}\right) \delta 2.66$ (br m, $\left.2 \mathrm{H}, \mathrm{CH}_{2} \mathrm{C} \equiv \mathrm{C}\right), 2.40(\mathrm{~d}, J=$ $\left.13.5 \mathrm{~Hz}, 1 \mathrm{H}, \mathrm{CH}_{2} \mathrm{C}\left(\mathrm{CH}_{3}\right)_{2}\right), 2.01\left(\mathrm{~d}, \mathrm{~J}=13.4 \mathrm{~Hz}, 1 \mathrm{H}, \mathrm{CH}_{2} \mathrm{C}\left(\mathrm{CH}_{3}\right)_{2}\right), 1.57\left(\mathrm{~s}, 3 \mathrm{H}, \mathrm{OCCH}_{3}\right), 1.37(\mathrm{~s}, 3 \mathrm{H}$, $\left.\mathrm{O}=\mathrm{CC}\left(\mathrm{CH}_{3}\right)_{2}\right), 1.34\left(\mathrm{~s}, 3 \mathrm{H}, \mathrm{O}=\mathrm{CC}\left(\mathrm{CH}_{3}\right)_{2}\right), 1.13-1.05(\mathrm{~m}, 21 \mathrm{H}, \mathrm{TIPS}) .{ }^{13} \mathrm{C} \mathrm{NMR}\left(100 \mathrm{MHz}, \mathrm{CDCl}_{3}\right) \delta 181.5$, 102.8, 83.9, 81.2, 46.4, 40.8, 34.2, 27.8, 27.7, 27.0 18.6, 11.2. IR v 2943 (s), 2894 (m), 2866 (s), 2176 (w), 1774 
(s), $1463(\mathrm{~m}), 1384(\mathrm{w}), 1290(\mathrm{w}), 1231(\mathrm{~m}), 1203(\mathrm{w}), 1163(\mathrm{w}), 1089(\mathrm{~m}), 1035(\mathrm{w}), 995(\mathrm{w}), 954(\mathrm{w}), 954$ (w), $920(\mathrm{w}), 883(\mathrm{~m}), 753(\mathrm{w}), 728(\mathrm{w}), 677$ (s), $620(\mathrm{~m})$. HRMS (ESI) expected for $\mathrm{C}_{19} \mathrm{H}_{35} \mathrm{O}_{2} \mathrm{Si}^{+}(\mathrm{M}+\mathrm{H})$ 323.2406; found: 323.2410 .

\section{5-Methyl-5-(3-(triisopropylsilyl)prop-2-ynyl)dihydrofuran-2(3H)-one (10b)}

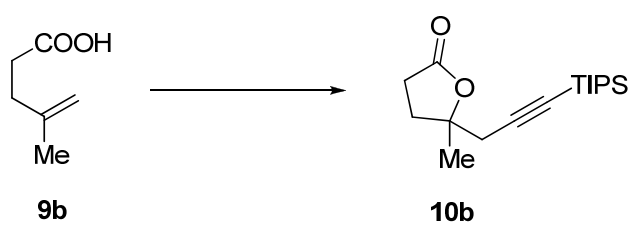

Column chromatography $\left(\mathrm{SiO}_{2}, \mathrm{PET} /\right.$ EtOAc 97:3) afforded product 10b $(86.5 \mathrm{mg}, 0.287 \mathrm{mmol}, 72 \%$ yield) as a yellow solid. $\mathrm{R}_{\mathrm{f}} 0.57$ (PET/EtOAc 4: 1). Mp $47-49^{\circ} \mathrm{C} .{ }^{1} \mathrm{H}$ NMR $\left(400 \mathrm{MHz}, \mathrm{CDCl}_{3}\right) \delta 2.77-2.54(\mathrm{~m}, 4 \mathrm{H}$, $\left.\mathrm{CH}_{2} \mathrm{C} \equiv \mathrm{C}, \mathrm{CH}_{2} \mathrm{C}=\mathrm{O}\right), 2.41\left(\mathrm{ddd}, J=13.0,10.1,6.8 \mathrm{~Hz}, 1 \mathrm{H}, \mathrm{CH}_{2} \mathrm{C}\left(\mathrm{CH}_{3}\right)\right), 2.05(\mathrm{ddd}, J=13.0,9.7,7.2 \mathrm{~Hz}, 1 \mathrm{H}$, $\left.\mathrm{CH}_{2} \mathrm{C}\left(\mathrm{CH}_{3}\right)\right), \quad 1.51\left(\mathrm{~s}, 3 \mathrm{H}, \mathrm{CH}_{3}\right), 1.10-1.01(\mathrm{~m}, 21 \mathrm{H}, \quad \mathrm{TIPS}) .{ }^{13} \mathrm{C}$ NMR $\left(100 \mathrm{MHz} \mathrm{CDCl}_{3}\right)$ $\delta$ 176.2, 102.6, 84.9, 84.1, 32.5, 32.0, 29.1, 26.1, 18.6, 11.2. IR v 2943 (s), 2892 (m), 2866 (s), 2175 (m), 1778 (s), 1463 (m), 1419 (w), 1385 (w), 1295 (w), 1277 (w), 1248 (w), $1196(\mathrm{~s}), 1172$ (w), 1117 (m), 1087 (m), 1037 (m), 1013 (w), 994 (w), 946 (m), 918 (w), 884 (m), 735 (w), 677 (s). HRMS (ESI) expected for $\mathrm{C}_{17} \mathrm{H}_{31} \mathrm{O}_{2} \mathrm{Si}^{+}$ $(\mathrm{M}+\mathrm{H})$ 295.2093; found: 295.2079.

\section{3,3-Dimethyl-5-(3-(triisopropylsilyl)prop-2-ynyl)dihydrofuran-2(3H)-one (10c)}

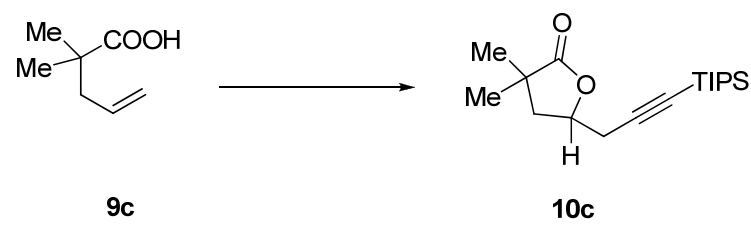

Column chromatography $\left(\mathrm{SiO}_{2}, \mathrm{PET} / \mathrm{DCM} /\right.$ EtOAc 96: 3: 1) afforded product 10c (94 mg, $0.30 \mathrm{mmol}, 76 \%$ yield) as a yellow oil. $\mathrm{R}_{\mathrm{f}} 0.39$ (PET/EtOAc 10: 1). ${ }^{1} \mathrm{H}$ NMR $\left(400 \mathrm{MHz}, \mathrm{CDCl}_{3}\right) \delta 4.54(\mathrm{~m}, 1 \mathrm{H}, \mathrm{CH}), 2.75(\mathrm{dd}, J$ $\left.=16.9,4.4 \mathrm{~Hz}, 1 \mathrm{H}, \mathrm{CH}_{2} \mathrm{C} \equiv \mathrm{C}\right), 2.66\left(\mathrm{dd}, \mathrm{J}=16.9,7.0 \mathrm{~Hz}, 1 \mathrm{H}, \mathrm{CH}_{2} \mathrm{C} \equiv \mathrm{C}\right), 2.19(\mathrm{dd}, J=12.8,6.1 \mathrm{~Hz}, 1 \mathrm{H}$, $\left.\mathrm{CH}_{2} \mathrm{C}\left(\mathrm{CH}_{3}\right)_{2}\right), 2.05\left(\mathrm{dd}, J=12.8,9.7 \mathrm{~Hz}, 1 \mathrm{H}, \mathrm{CH}_{2} \mathrm{C}\left(\mathrm{CH}_{3}\right)_{2}\right), 1.29\left(\mathrm{~s}, 3 \mathrm{H}, \mathrm{CH}_{3}\right), 1.26\left(\mathrm{~s}, 3 \mathrm{H}, \mathrm{CH}_{3}\right), 1.08-1.01$ $(\mathrm{m}, \quad 21 \quad \mathrm{H}, \quad$ TIPS $) . \quad{ }^{13} \mathrm{C} \quad \mathrm{NMR} \quad\left(100 \quad \mathrm{MHz}, \quad \mathrm{CDCl}_{3}\right)$ $\delta$ 181.3, 101.6, 84.2, 74.1, 42.0, 40.4, 26.2, 24.8, 24.7, 18.6, 11.1. IR v $2943(\mathrm{~m}), 2893(\mathrm{w}), 2866(\mathrm{~m}), 2178(\mathrm{w})$, 1777 (s), 1464 (m), 1384 (w), 1349 (w), 1231 (w), 1206 (m), 1147 (m), 1121 (m), 1074 (w), 1037, 1016 (m), $918(\mathrm{~m}), 884(\mathrm{~m}), 739$ (w), 676 (s), 629 (m). HRMS (ESI) expected for $\mathrm{C}_{18} \mathrm{H}_{33} \mathrm{O}_{2} \mathrm{Si}^{+}$(M+H) 309.2250; found: 309.2251 .

\section{5-(3-(triisopropylsilyl)prop-2-ynyl)dihydrofuran-2(3H)-one (10d)}




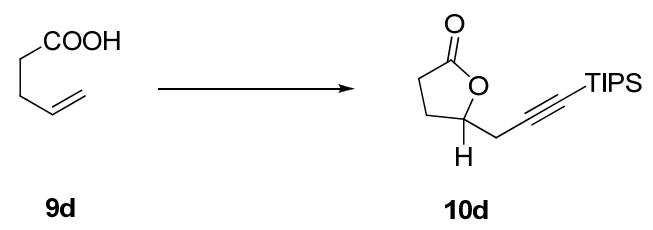

Column chromatography $\left(\mathrm{SiO}_{2}\right.$, PET/EtOAc 95: 5) afforded product 10d (92 mg, $0.33 \mathrm{mmol}, 82 \%$ yield 95\% purity according to ${ }^{1} \mathrm{H}-\mathrm{NMR}$ ) as a yellow oil. $\mathrm{R}_{\mathrm{f}} 0.35$ (PET/EtOAc 4:1). ${ }^{1} \mathrm{H}$ NMR $\left(400 \mathrm{MHz}, \mathrm{CDCl}_{3}\right) \delta$ 4.66 (qd, $J=7.0,4.4 \mathrm{~Hz}, 1 \mathrm{H}, \mathrm{CH}), 2.85-2.51\left(\mathrm{~m}, 4 \mathrm{H}, \mathrm{CH}_{2} \mathrm{C}=\mathrm{O}, \mathrm{CH}_{2} \mathrm{C} \equiv \mathrm{C}\right), 2.43\left(\mathrm{~m}, 1 \mathrm{H}, \mathrm{CH}_{2}\right), 2.20$ (m, $1 \mathrm{H}$, $\mathrm{CH}_{2}$ ), 1.20-0.95 (m, $\left.21 \mathrm{H}, \mathrm{TIPS}\right) .{ }^{13} \mathrm{C}$ NMR $\left(100 \mathrm{MHz}, \mathrm{CDCl}_{3}\right) \delta$ 176.7, 101.6, 84.3, 77.7, 28.4, 26.52, 26.5, 18.5, 11.0. IR $\vee 2943$ (s), 2894 (m), 2866 (s), 2176 (w), 1781 (s), 1463 (m), 1382 (w), 1352 (w), 1291 (w), 1239 (w), 1177 (s), 1150 (sh), 1049 (sh), 1019 (s), 998 (m), 919 (m), 884 (s), 839 (w), 797 (w), 732 (m), 678 (s), 660 (s), 632 (m). HRMS (ESI) expected for $\mathrm{C}_{16} \mathrm{H}_{29} \mathrm{O}_{2} \mathrm{Si}^{+}$(M+H) 281.1937; found: 281.1939.

\section{Mechanistic Investigations}

\section{Stoichiometric NMR investigations}

Experiment 1: Reagent 3d (17 mg, $0.040 \mathrm{mmol}, 1.0$ equiv) was added to a solution of Pd (hfacac) $2(21 \mathrm{mg}$, 0.040 mmol, 1.0 equiv) in $\mathrm{CD}_{2} \mathrm{Cl}_{2}(1 \mathrm{~mL}) .{ }^{1} \mathrm{H}-\mathrm{NMR}$ spectra were acquired $10 \mathrm{~min}, 40 \mathrm{~min}$ and $3 \mathrm{~h}$ after mixing, whereas full decomposition of $\mathbf{3 d}$ was observed.

Experiment 2: Methylallyl-phenol (1a) (6 mg, $0.04 \mathrm{mmol}, 1$ equiv) was added to a solution of $\mathrm{Pd}$ (hfacac) $)_{2}(21$ $\mathrm{mg}, 0.040 \mathrm{mmol}, 1.0$ equiv) in $\mathrm{CD}_{2} \mathrm{Cl}_{2}(1 \mathrm{~mL}) .{ }^{1} \mathrm{H}-\mathrm{NMR}$ spectra were acquired $10 \mathrm{~min}, 40 \mathrm{~min}$ and $8 \mathrm{~h}$ after mixing. Partially isomerization of the double bond into conjugation was observed.

Experiment 3: Reagent 3d (17 mg, $0.040 \mathrm{mmol}, 1.0$ equiv) was added to a solution of Pd (hfacac) $2(21 \mathrm{mg}$, 0.040 mmol, 1.0 equiv) in $\mathrm{CD}_{2} \mathrm{Cl}_{2}(1 \mathrm{~mL})$. After 6 min Methylallyl phenol (1a) $(6 \mathrm{mg}, 0.04 \mathrm{mmol}, 1$ equiv) was added. ${ }^{1}$ H-NMR spectra were acquired 5 minutes and 2 hours after the addition of 3d. After 2 hours complete conversion to product $\mathbf{4 b}$ was observed.

Experiment 4: Methylallyl phenol 1a (6 $\mathrm{mg}, 0.04 \mathrm{mmol}, 1$ equiv) was added to a solution of Pd (hfacac) $)_{2}(21$ $\mathrm{mg}, 0.040 \mathrm{mmol}, 1.0$ equiv) in $\mathrm{CD}_{2} \mathrm{Cl}_{2}(1 \mathrm{~mL})$. After $50 \mathrm{~min},{ }^{1} \mathrm{H}-\mathrm{NMR}$ showed partial isomerization of the double bond. After $55 \mathrm{~min}$ reagent $3 \mathbf{d}\left(17 \mathrm{mg}, 0.040 \mathrm{mmol}, 1.0\right.$ equiv) was added. ${ }^{1} \mathrm{H}-\mathrm{NMR}$ spectra were acquired 5 minutes and 2 hours after the addition of $\mathbf{3 d}$. After 2 hours complete conversion to product $\mathbf{4 b}$ was observed.

\section{Preparation and reaction of ${ }^{13} \mathrm{C}$-labeled reagent $3 d^{\prime}$}

\section{2-(4-Methoxybenzyloxy)ethanol (55)}

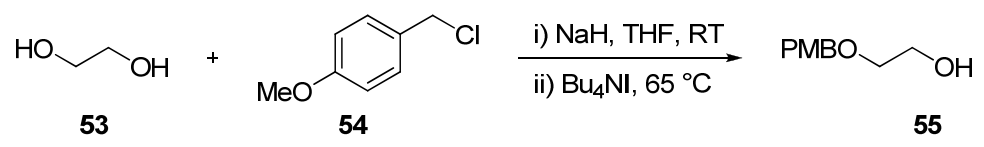


Following a reported procedure, ${ }^{27}$ sodium hydride $(60 \%$ in mineral oil, $0.70 \mathrm{~g}, 17 \mathrm{mmol}, 1.0$ equiv) was added to a solution of ethylene glycol (53) (freshly distilled from drierite $\left(\mathrm{p}=0.3 \mathrm{mbar}, \mathrm{T}=46{ }^{\circ} \mathrm{C}\right), 2.8 \mathrm{~mL}, 50$ mmol, 3.0 equiv) in THF (30 mL). After stirring $30 \mathrm{~min}$ at RT, 4-methoxybenzyl chloride (54) (2.60 g, 16.6 mmol, 1.00 equiv) and $\mathrm{Bu}_{4} \mathrm{NI}(0.61 \mathrm{~g}, 1.7 \mathrm{mmol}, 0.10$ equiv) were added, and the reaction mixture was heated to reflux. After $4.5 \mathrm{~h}$, the reaction mixture was cooled to RT, the reaction was quenched with sat. $\mathrm{NH}_{4} \mathrm{Cl}(30$ $\mathrm{mL})$ and extracted with $\mathrm{Et}_{2} \mathrm{O}(3 \times 30 \mathrm{~mL})$. The combined organic layers were washed with brine $(30 \mathrm{~mL})$, dried over $\mathrm{MgSO}_{4}$ and the solvent was removed under reduced pressure. The crude product was purified by flash column chromatography (PET/AcOEt 5/1-1/1) to yield protected alcohol 55 (2.54 g, $13.9 \mathrm{mmol}, 84 \%)$ as a yellow oil. $R_{f}\left(\mathrm{PET} / \mathrm{AcOEt} 1 / 1, \mathrm{KMnO}_{4}\right)$ 0.25. ${ }^{1} \mathrm{H} \mathrm{NMR}\left(\mathrm{CDCl}_{3}, 400 \mathrm{MHz}\right) \delta 7.27(\mathrm{dm}, J=8.2 \mathrm{~Hz}, 2 \mathrm{H}, \mathrm{ArH})$, $6.89(\mathrm{dm}, J=8.6 \mathrm{~Hz}, 2 \mathrm{H}, \mathrm{ArH}), 4.49\left(\mathrm{~s}, 2 \mathrm{H}\right.$, benzyl $\left.\mathrm{CH}_{2}\right), 3.81\left(\mathrm{~s}, 3 \mathrm{H}, \mathrm{OCH}_{3}\right), 3.74(\mathrm{t}, J=4.3 \mathrm{~Hz}, 2 \mathrm{H}$, $\mathrm{CH}_{2} \mathrm{OPMB}$ ), 3.57 (m, $\left.2 \mathrm{H}, \mathrm{CH}_{2} \mathrm{OH}\right), 2.08$ (br s, $\left.1 \mathrm{H}, \mathrm{OH}\right) .{ }^{1} \mathrm{H}$ NMR corresponded to the literature values. ${ }^{22}$

\section{2-(4-Methoxybenzyloxy)acetaldehyde (57)}

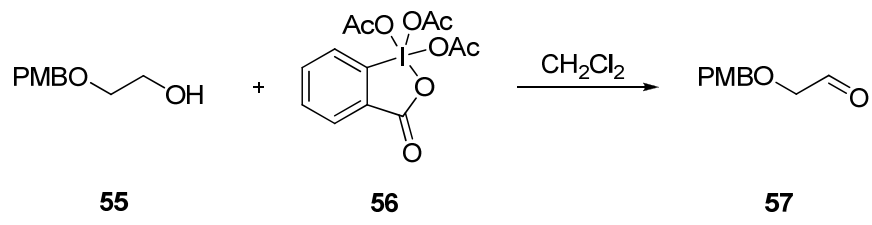

Following a reported procedure, ${ }^{28}$ Dess-Martin Periodinane (56) (0.53 g, $1.3 \mathrm{mmol}, 1.1$ equiv) was added to a solution of alcohol $55\left(0.21 \mathrm{~g}, 1.2 \mathrm{mmol}, 1.0\right.$ equiv) in wet $\mathrm{CH}_{2} \mathrm{Cl}_{2}(9 \mathrm{~mL})$. After stirring $2.5 \mathrm{~h}$ at $\mathrm{RT}$, the reaction was quenched with sat. $\mathrm{NaHCO}_{3}(10 \mathrm{~mL})$ and sat. sodium thiosulfate solution $(10 \mathrm{~mL})$ and the mixture was stirred vigorously for 10 min until two clear layers were obtained. The layers were separated and the water layer was extracted with $\mathrm{CH}_{2} \mathrm{Cl}_{2}(3 \times 20 \mathrm{~mL})$. The combined organic layers were dried over $\mathrm{MgSO}_{4}$ and the solvent was removed under reduced pressure to give aldehyde 57 (0.21 g, $1.2 \mathrm{mmol}$, quant.) as a yellow oil, which was used immediately in the next step without further purification. $R_{f}\left(\mathrm{PET} / \mathrm{AcOEt} 1 / 1, \mathrm{KMnO}_{4}\right) 0.35$. ${ }^{1} \mathrm{H}$ NMR $\left(\mathrm{CDCl}_{3}, 400 \mathrm{MHz}\right) \delta 9.71(\mathrm{t}, J=0.9 \mathrm{~Hz}, \mathrm{CHO}), 7.29(\mathrm{dm}, J=8.8 \mathrm{~Hz}, 2 \mathrm{H}, \mathrm{ArH}), 6.90(\mathrm{dm}, J=8.8$ $\mathrm{Hz}, 2 \mathrm{H}, \mathrm{ArH}), 4.57$ (s, $2 \mathrm{H}$, benzyl $\mathrm{CH}_{2}$ ), 4.07 (d, J=0.9 Hz, $\left.2 \mathrm{H}, \mathrm{CH}_{2} \mathrm{CHO}\right), 3.81$ (s, $\left.3 \mathrm{H}, \mathrm{OCH}_{3}\right) .{ }^{1} \mathrm{H} \mathrm{NMR}^{\mathrm{N}}$ corresponded to the literature values. ${ }^{27}$

\section{Labeled 1-((3,3-dibromoallyloxy)methyl)-4-methoxybenzene \\ (60) and triisopropyl(3-(4-} methoxybenzyloxy)prop-1-ynyl)silane (61)

(27) Masutani, K.; Minowa, T.; Hagiwara, Y.; Mukaiyama, T. Bull. Chem. Soc. Jpn. 2006, 79, 1106.

(28) Collins, I.; Caldwell, J.; Fonseca, T.; Donald, A.; Bavetsias, V.; Hunter, L. J. K.; Garrett, M. D.; Rowlands, M. G.; Aherne, G. W.; Davies, T. G.; Berdini, V.; Woodhead, S. J.; Davis, D.; Seavers, L. C. A.; Wyatt, P. G.; Workman, P.; McDonald, E. Bioorg. Med. Chem. 2006, 14, 1255. 

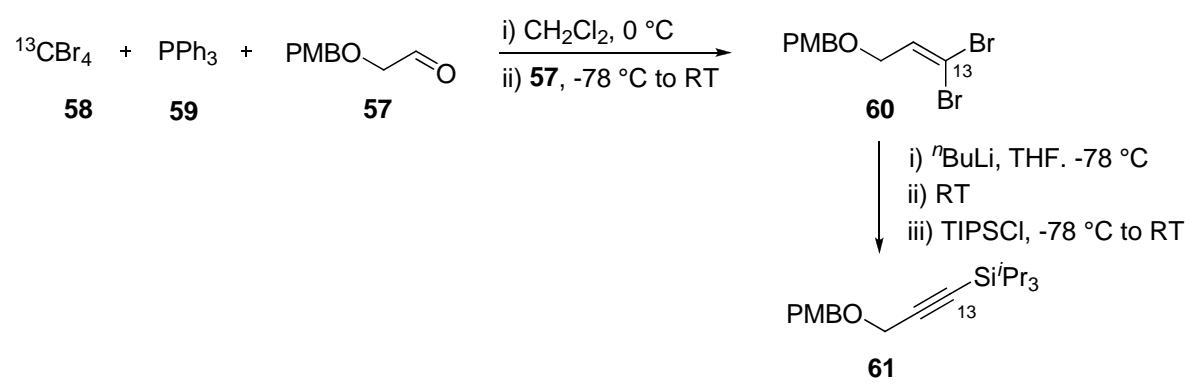

Following a slightly modified literature procedure, ${ }^{29}$ a solution of $\mathrm{PPh}_{3}(57)(1.6 \mathrm{~g}, 6.0 \mathrm{mmol}, 2.0$ equiv) in $\mathrm{CH}_{2} \mathrm{Cl}_{2}(9 \mathrm{~mL})$ was added to a solution of $\mathrm{CBr}_{4}(58)\left(1.0 \mathrm{~g}, 3.0 \mathrm{mmol}, 1.0\right.$ equiv, $20 \%{ }^{13} \mathrm{C}$, prepared from $0.80 \mathrm{~g}$ natural $\mathrm{CBr}_{4}$ and $0.20 \mathrm{~g} \mathrm{99 \%}{ }^{13} \mathrm{C}$-enriched $\left.\mathrm{CBr}_{4}\right)$ in $\mathrm{CH}_{2} \mathrm{Cl}_{2}(12 \mathrm{~mL})$ at $0{ }^{\circ} \mathrm{C}$ over $15 \mathrm{~min}$. After stirring for 15 min at $0{ }^{\circ} \mathrm{C}$, the yellow-orange solution was cooled to $-78{ }^{\circ} \mathrm{C}$ and a solution of aldehyde 57 (freshly synthesized, $0.66 \mathrm{~g}, 3.6 \mathrm{mmol}, 1.2$ equiv) in $\mathrm{CH}_{2} \mathrm{Cl}_{2}(9 \mathrm{~mL})$ was added over $10 \mathrm{~min}$, whereas the reaction mixture turned dark red-brown. The reaction mixture was left to warm to RT over $17 \mathrm{~h}$, quenched with sat. $\mathrm{NaHCO}_{3}(30 \mathrm{~mL})$ and extracted with $\mathrm{CH}_{2} \mathrm{Cl}_{2}(3 \times 30 \mathrm{~mL})$. The combined organic layers were washed with brine $(30 \mathrm{~mL})$, dried over $\mathrm{MgSO}_{4}$ and the solvent was removed under reduced pressure. The crude product was purified by flash column chromatography ( $\left.\mathrm{PET} / \mathrm{CH}_{2} \mathrm{Cl}_{2} 3 / 1-1.5 / 1\right)$ to yield dibromide 60 (501 g, $1.55 \mathrm{mmol}$, $52 \%)$ as a slightly yellow oil, which was used directly in the next step. $R_{f}\left(\mathrm{PET} / \mathrm{CH}_{2} \mathrm{Cl}_{2} 2 / 1, \mathrm{KMnO}_{4}\right) 0.30 .{ }^{1} \mathrm{H}$ NMR $\left(\mathrm{CDCl}_{3}, 400 \mathrm{MHz}\right) \delta 7.28(\mathrm{dm}, J=8.6 \mathrm{~Hz}, 2 \mathrm{H}, \mathrm{ArH}), 6.90(\mathrm{dm}, J=8.6 \mathrm{~Hz}, 2 \mathrm{H}, \mathrm{ArH}), 6.64(\mathrm{tm}, J=6.1$ $\mathrm{Hz}, 1 \mathrm{H}$, alkene $\mathrm{H}), 4.46\left(\mathrm{~s}, 2 \mathrm{H}\right.$, benzyl $\left.\mathrm{CH}_{2}\right), 4.04\left(\mathrm{~m}, 2 \mathrm{H}\right.$, alkene $\left.\mathrm{CH}_{2}\right), 3.81\left(\mathrm{~s}, 3 \mathrm{H}, \mathrm{OCH}_{3}\right) .{ }^{13} \mathrm{C} \mathrm{NMR}$ $\left(\mathrm{CDCl}_{3}, 100 \mathrm{MHz}\right) \delta 159.3,135.6,129.5,129.4,113.8,91.3$ (labeled 20x more intensive), 72.2, 69.4, 55.2.

Following a literature procedure, ${ }^{30}{ }^{n} \mathrm{BuLi}(2.5 \mathrm{M}$ in hexane, $1.4 \mathrm{~mL}, 3.4 \mathrm{mmol}, 2.2$ equiv) was added dropwise to a solution of dibromide $60\left(0.50 \mathrm{~g}, 1.5 \mathrm{mmol}, 1.0\right.$ equiv) in THF $(9 \mathrm{~mL})$ at $-78{ }^{\circ} \mathrm{C}$. The yellow solution was stirred $1 \mathrm{~h}$ at $-78{ }^{\circ} \mathrm{C}$ and $1 \mathrm{~h}$ at RT. After cooling to $-78{ }^{\circ} \mathrm{C}$, TIPSCl $(0.43 \mathrm{~mL}, 2.0 \mathrm{mmol}, 1.3$ equiv) was added and the reaction was left to warm to RT over $12 \mathrm{~h}$. The reaction was quenched with sat. $\mathrm{NaHCO}_{3}(10 \mathrm{~mL})$ and extracted with $\mathrm{Et}_{2} \mathrm{O}(3 \times 20 \mathrm{~mL})$. The combined organic layers were washed with brine $(10 \mathrm{~mL})$, dried over $\mathrm{MgSO}_{4}$ and the solvent was removed under reduced pressure. The crude product was purified by flash column chromatography ( $\mathrm{PET} / \mathrm{CH}_{2} \mathrm{Cl}_{2}$ 4/1-2/1) to yield alkyne 61 (372 g, $\left.1.12 \mathrm{mmol}, 72 \%\right)$ as a colorless oil. Comparison of the ${ }^{13} \mathrm{CNMR}$ with an unlabeled sample (synthesized following the same procedure) showed $20 \%{ }^{13} \mathrm{C}$ incorporation at the indicated position only. $R_{f}\left(\mathrm{PET} / \mathrm{CH}_{2} \mathrm{Cl}_{2} 2 / 1, \mathrm{KMnO}_{4}\right) 0.35$. ${ }^{1} \mathrm{H} \mathrm{NMR}\left(\mathrm{CDCl}_{3}, 400 \mathrm{MHz}\right) \delta 7.30(\mathrm{dm}, J=8.4 \mathrm{~Hz}, 2 \mathrm{H}, \mathrm{ArH}), 6.89(\mathrm{dm}, J=8.6 \mathrm{~Hz}, 2 \mathrm{H}, \operatorname{ArH}), 4.59(\mathrm{~s}, 2 \mathrm{H}$, benzyl $\left.\mathrm{CH}_{2}\right), 4.19$ (s, $2 \mathrm{H}$, alkyne $\left.\mathrm{CH}_{2}\right), 3.81\left(\mathrm{~s}, 3 \mathrm{H}, \mathrm{OCH}_{3}\right), 1.12$ (m, $21 \mathrm{H}$, TIPS). ${ }^{13} \mathrm{C}$ NMR $\left(\mathrm{CDCl}_{3}, 100\right.$ MHz) $\delta 159.3,129.8,129.5,113.8,103.4,87.7$ (labeled 20x more intensive), 70.6, 57.4, 55.2, 18.6, 11.1. IR v $2961(w), 2944$ (w), 2931 (w), 2866 (w), 2171 (w), 1663 (w), 1614 (w), 1515 (w), 1463 (w), 1444 (w), 1378

(29) Paquette, L. A.; Chang, J. Y.; Liu, Z. S. J. Org. Chem. 2004, 69, 6441.

(30) Corey, E. J.; Fuchs, P. L. Tetrahedron Lett. 1972, 3769. 
(w), $1250(\mathrm{w}), 1078(\mathrm{~m}), 1036(\mathrm{w}), 907$ (s), 730 (s), 651 (m). HRMS(ESI) calcd for $\mathrm{C}_{15} \mathrm{H}_{16} \mathrm{O}_{3}{ }^{+}(\mathrm{M}+\mathrm{H})$

333.2250, found 333.2254. Isotope repartition: expected for about 20\% incorporation was obtained.

\section{Labeled triisopropyl acetylene (17’)}

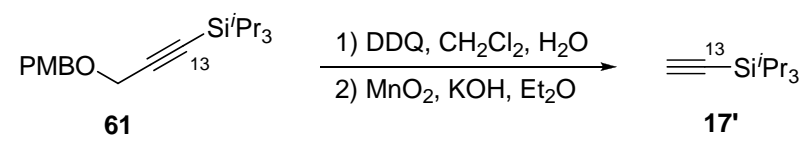

Following a literature procedure, ${ }^{31}$ DDQ $(0.38 \mathrm{~g}, 1.7 \mathrm{mmol}, 1.5$ equiv $)$ was added to a solution of protected alcohol 61 (372 mg, $1.12 \mathrm{mmol}, 1.00$ equiv) in $\mathrm{CH}_{2} \mathrm{Cl}_{2}(11 \mathrm{~mL})$ and water $(1.1 \mathrm{~mL})$ at $0{ }^{\circ} \mathrm{C}$. The reaction mixture was stirred $15 \mathrm{~min}$ at $0{ }^{\circ} \mathrm{C}$ and $3 \mathrm{~h}$ at $\mathrm{RT}$. The resulting dark red thick suspension was quenched with sat. $\mathrm{NaHCO}_{3}(20 \mathrm{~mL})$ and extracted with $\mathrm{CH}_{2} \mathrm{Cl}_{2}(3 \times 20 \mathrm{~mL})$. The combined organic layers were washed with sat. $\mathrm{NaHCO}_{3}(20 \mathrm{~mL})$ and brine $(20 \mathrm{~mL})$, dried over $\mathrm{MgSO}_{4}$ and the solvent was removed under reduced pressure. TLC (PET/AcOEt 6/1, $\mathrm{KMnO}_{4}$ ) showed a mixture of two products, which were shown to be the corresponding propargylic alcohol $\left(\mathrm{R}_{\mathrm{f}}=0.50\right)$ and anisaldehyde $\left(\mathrm{R}_{\mathrm{f}}=0.45\right)$ by ${ }^{1} \mathrm{H}$ NMR. This mixture was directly used as such in the next step.

Following a literature procedure, ${ }^{32}$ the obtained mixture was diluted in $\mathrm{Et}_{2} \mathrm{O}(14 \mathrm{~mL})$ and $\mathrm{MnO}_{2}(\mathrm{Aldrich}$ activated, $1.2 \mathrm{~g}, 13 \mathrm{mmol}, 12$ equiv) and $\mathrm{KOH}$ (freshly grounded, $0.38 \mathrm{~g}, 6.8 \mathrm{mmol}, 6.0$ equiv) were added in 4 portions every hour. After stirring for further $3 \mathrm{~h}$, TLC (PET/AcOEt 6/1, $\mathrm{KMnO}_{4}$ ) showed complete conversion and the reaction mixture was filtered over $\mathrm{SiO}_{2}$ and the filter cake was washed with $\mathrm{Et}_{2} \mathrm{O}(50 \mathrm{~mL})$. The solvent was removed under reduced pressure and the crude mixture was purified by flash column chromatography (PET) to yield alkyne 17' (167 mg, $0.915 \mathrm{mmol}, 82 \%)$ as a colorless oil. Comparison of the ${ }^{13} \mathrm{CNMR}$ with an unlabeled sample (synthesized following the same procedure) showed $20 \%{ }^{13} \mathrm{C}$ incorporation at the indicated position only. $R_{f}\left(\mathrm{PET}, \mathrm{KMnO}_{4}\right)$ 0.80. ${ }^{1} \mathrm{H} \mathrm{NMR}\left(\mathrm{CD}_{2} \mathrm{Cl}_{2}, 400 \mathrm{MHz}\right) \delta 2.38(\mathrm{~s}, 1 \mathrm{H}$, alkyne $\mathrm{H}), 1.07(\mathrm{~m}, 21 \mathrm{H}$, TIPS). ${ }^{13} \mathrm{C} \mathrm{NMR}\left(\mathrm{CD}_{2} \mathrm{Cl}_{2}, 100 \mathrm{MHz}\right) \delta 94.9,86.2$ (labeled 20x more intensive), 18.3, 11.1.

\section{Labeled triisopropyl((trimethylsilyl)ethynyl)silane (15’)}

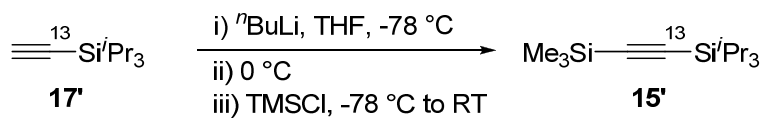

${ }^{n} \operatorname{BuLi}(2.5 \mathrm{M}$ in hexane, $0.44 \mathrm{~mL}, 1.1 \mathrm{mmol}, 1.2$ equiv) was added to a solution of acetylene 17’ (167 mg, $0.915 \mathrm{mmol}, 1.00$ equiv) in THF $(2 \mathrm{~mL})$ at $-78{ }^{\circ} \mathrm{C}$. The reaction mixture was stirred $15 \mathrm{~min}$ at $0{ }^{\circ} \mathrm{C}$ and the yellow solution was cooled back to $-78^{\circ} \mathrm{C}$. $\mathrm{TMSCl}$ (freshly distilled, $0.15 \mathrm{~mL}, 1.2 \mathrm{mmol}, 1.3$ equiv) was added and the colorless solution was left to warm to RT over $6 \mathrm{~h}$. The reaction was quenched with sat. $\mathrm{NH}_{4} \mathrm{Cl}(3 \mathrm{~mL})$ and extracted with $\mathrm{Et}_{2} \mathrm{O}(3 \times 10 \mathrm{~mL})$. The combined organic layers were washed with brine $(5 \mathrm{~mL})$, dried over

(31) Dimopoulos, P.; Athlan, A.; Manaviazar, S.; George, J.; Walters, M.; Lazarides, L.; Aliev, A. E.; Hale, K. J. Org. Lett. 2005, 7, 5369.

(32) Kukula, H.; Veit, S.; Godt, A. Eur. J. Org. Chem. 1999, 277. 
$\mathrm{MgSO}_{4}$ and the solvent was removed under reduced pressure. The crude mixture was purified by flash column chromatography (PET) to yield protected alkyne 15’ (184 g, $0.722 \mathrm{mmol}, 79 \%)$ as a colorless oil. Comparison of the ${ }^{13} \mathrm{C}$ NMR with an unlabeled sample showed $20 \%{ }^{13} \mathrm{C}$ incorporation at the indicated position only. $R_{f}$ $\left(\mathrm{PET}, \mathrm{KMnO}_{4}\right)$ 0.80. ${ }^{1} \mathrm{H} \mathrm{NMR}\left(\mathrm{CDCl}_{3}, 400 \mathrm{MHz}\right) \delta 1.07$ (m, $\left.21 \mathrm{H}, \mathrm{TIPS}\right), 0.17$ (s, $\left.9 \mathrm{H}, \mathrm{TMS}\right) .{ }^{13} \mathrm{C} \mathrm{NMR}$ $\left(\mathrm{CDCl}_{3}, 100 \mathrm{MHz}\right) \delta 116.2,110.1$ (labeled 20x more intensive), 18.6, 11.1, 0.0. IR v $2959(\mathrm{~m}), 2944$ (m), 2896 (w), 2867 (m), 1464 (w), 1385 (w), 1250 (m), 996 (w), 842 (s), 764 (s), 675 (m), 660 (m). ${ }^{1} \mathrm{H} \mathrm{NMR}$ corresponded to the literature values. ${ }^{4}$

\section{Labeled 1-[(triisopropyllsilyl)ethynyl]-1,2-benziodoxol-3(1H)-one (3d')}

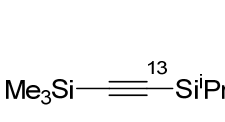

15'

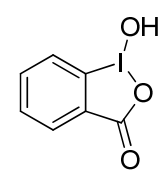

13

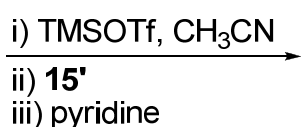

iii) pyridine

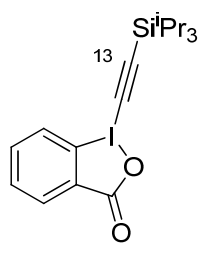

3d'

Following a slightly modified literature procedure, ${ }^{3}$ TMSOTf (freshly distilled, $0.15 \mathrm{~mL}, 0.82 \mathrm{mmol}, 1.1$ equiv) was added to a suspension of iodinane 13 (freshly synthesized, $0.19 \mathrm{~g}, 0.72 \mathrm{mmol}, 1.0$ equiv) in $\mathrm{CH}_{3} \mathrm{CN}$ $(6.5 \mathrm{~mL})$. After $10 \mathrm{~min}$, a solution of acetylene 15’ $\left(0.18 \mathrm{~g}, 0.72 \mathrm{mmol}, 1.0\right.$ equiv) in $\mathrm{CH}_{2} \mathrm{Cl}_{2}(1.0 \mathrm{~mL})$ was added to the slightly yellow solution. After stirring $15 \mathrm{~min}$ at $\mathrm{RT}$, pyridine ( $70 \mu \mathrm{L}, 0.87 \mathrm{mmol}, 1.2$ equiv) was added and the solvent was removed under reduced pressure below $30{ }^{\circ} \mathrm{C}$. The reaction mixture was diluted with $\mathrm{CH}_{2} \mathrm{Cl}_{2}(15 \mathrm{~mL})$ and washed with $1 \mathrm{M} \mathrm{HCl}(5 \mathrm{~mL})$. The water layer was extracted with $\mathrm{CH}_{2} \mathrm{Cl}_{2}(2 \times 5 \mathrm{~mL})$ and the combined organic layers were washed with sat. $\mathrm{Na}_{2} \mathrm{CO}_{3}(2 \times 10 \mathrm{~mL})$. The combined basic aqueous layers were extracted with $\mathrm{CH}_{2} \mathrm{Cl}_{2}(10 \mathrm{~mL})$ and the combined organic layers were dried over $\mathrm{MgSO}_{4}$ and the solvent was removed under reduced pressure to give iodinane 3d' $\left(>95 \%\right.$ pure by ${ }^{1} \mathrm{HNMR}$, containing traces of acetylene 15’, $259 \mathrm{mg}, 0.604 \mathrm{mmol}, 84 \%$ ) as a slightly yellow solid. Comparison of the ${ }^{13} \mathrm{C}$ NMR with an unlabeled sample showed $20 \%{ }^{13} \mathrm{C}$ incorporation at the indicated position only. ${ }^{1} \mathrm{H} \mathrm{NMR}\left(\mathrm{CDCl}_{3}, 400 \mathrm{MHz}\right) \delta$ $8.40(\mathrm{~m}, 1 \mathrm{H}, \mathrm{Ar} \mathrm{H}), 8.28(\mathrm{~m}, 1 \mathrm{H}, \mathrm{Ar} \mathrm{H}), 7.74(\mathrm{~m}, 2 \mathrm{H}, \mathrm{Ar} \mathrm{H}), 1.13$ (m, $21 \mathrm{H}, \mathrm{TIPS}) .{ }^{13} \mathrm{C} \mathrm{NMR}\left(\mathrm{CDCl}_{3}, 100\right.$ MHz) $\delta 166.3,134.6,132.4,131.5,131.4,126.0,115.6,114.1$ (labeled 20x more intensive), 64.7, 18.4, 11.1.

\section{Labeled triisopropyl-[3-(2-methyl-2,3-dihydro-benzofuran-2-yl)-prop-1-ynyl]-silane (4b’)}

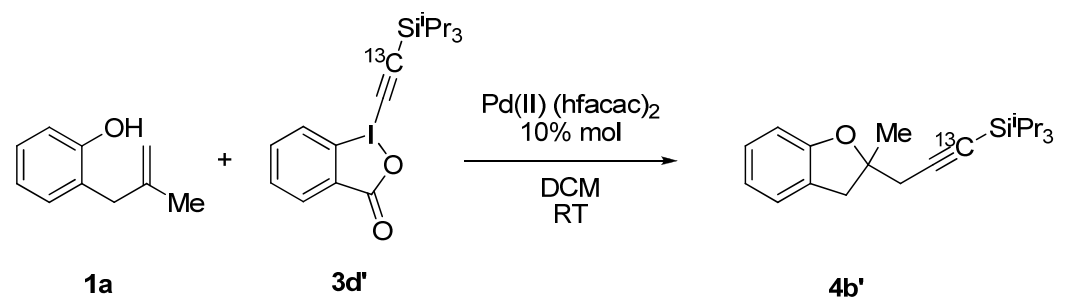

Palladium (II) hexafluoroacetylacetonate (11 $\mathrm{mg}, 0.020 \mathrm{mmol}, 0.10$ equiv.) was dissolved in dichloromethane (5 mL). 2-(Methylallyl)phenol (1a) (30 mg, $0.20 \mathrm{mmol}, 1.0$ equiv) was then added, followed 
by ${ }^{13} \mathrm{C}$ labeled 1-((triisopropylsilyl)ethynyl)-1,2-benziodoxol-3(1H)-one (3d') (1:1 mixture with non-labeled 3d: $0.10 \mathrm{~g}, 0.24 \mathrm{mmol}, 1.2$ equiv overall). The resulting solution was stirred under $\mathrm{N}_{2}$ at room temperature overnight. DCM was then removed under reduced pressure and the residue was treated with $\mathrm{NaHCO}_{3}$ (saturated solution, $6 \mathrm{~mL})$ and brine $(6 \mathrm{~mL})$. The mixture was extracted with diethyl ether $(3 \times 15 \mathrm{~mL})$ and the combined organic layers were dried over $\mathrm{MgSO}_{4}$, filtered and concentrated in vacuo. Column chromatography $\left(\mathrm{SiO}_{2}\right.$, PET/DCM 98:2) afforded product 4b' (51 mg, $0.15 \mathrm{mmol}, 75 \%$ yield) as a yellow oil. ${ }^{13} \mathrm{C} \mathrm{NMR}(100 \mathrm{MHz}$, $\left.\mathrm{CDCl}_{3}\right) \delta 158.7,128.0,126.6,125.1,120.2,109.5,104.3,87.4,82.9,40.6,32.5,26.2,18.6,11.3$. Intensity of peak at $\delta 82.9$ was increased approximately 10 times when compared with the corresponding peak for the nonlabeled compound $\mathbf{4 b}$.

\section{2-Methyl-2-(prop-2-ynyl)-2,3-dihydrobenzofuran (62)}

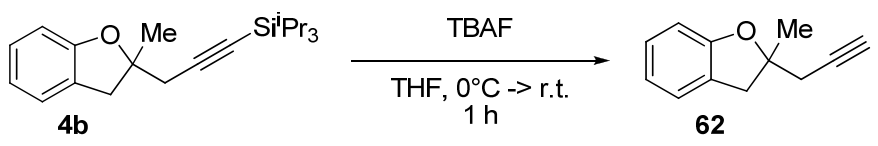

Following a reported procedure, ${ }^{33}$ triisopropyl-[3-(2-methyl-2,3-dihydro-benzofuran-2-yl)-prop-1-ynyl]silane (4b) (197 mg, $0.600 \mathrm{mmol}, 1.0$ equiv) was dissolved in THF (2.5 mL) and the resulting solution was stirred at $0{ }^{\circ} \mathrm{C}$ for $5 \mathrm{~min}$. TBAF ( $1 \mathrm{M}$ solution in THF, $1.2 \mathrm{~mL}, 1.2 \mathrm{mmol}, 2.0$ equiv) was then added and the mixture was stirred a $0{ }^{\circ} \mathrm{C}$ for $50 \mathrm{~min}$. The reaction mixture was allowed to warm to room temperature and stirred for $10 \mathrm{~min}$. $\mathrm{NH}_{4} \mathrm{Cl}$ (saturated solution, $25 \mathrm{~mL}$ ) was added followed by water $(17 \mathrm{~mL})$. The aqueous layer was extracted with $\mathrm{Et}_{2} \mathrm{O}(3 \mathrm{x} 20 \mathrm{~mL})$ and the combined organic layers were washed with $\mathrm{NH}_{4} \mathrm{Cl}(\mathrm{saturated}$ solution, $25 \mathrm{~mL})$ and brine $(25 \mathrm{~mL})$, dried over $\mathrm{MgSO}_{4}$, filtered and concentrated under reduced pressure. Purification by column chromatography $\left(\mathrm{SiO}_{2}\right.$, PET/EtOAc 99:1) afforded 2-methyl-2-(prop-2-ynyl)-2,3dihydrobenzofuran (62) (80 mg, $0.46 \mathrm{mmol}, 77 \%$ yield) as a colorless oil. $\mathrm{R}_{\mathrm{f}} 0.82(\mathrm{PET} / \mathrm{EtOAc} 10: 1) .{ }^{1} \mathrm{H} \mathrm{NMR}$ $\left(400 \mathrm{MHz} \mathrm{CDCl}_{3}\right) \oint 7.18-7.09(\mathrm{~m}, 2 \mathrm{H}, \mathrm{ArH}), 6.85(\mathrm{t}, J=7.4 \mathrm{~Hz}, 1 \mathrm{H}, \mathrm{ArH}), 6.77(\mathrm{~d}, J=8.1 \mathrm{~Hz}, 1 \mathrm{H}, \mathrm{ArH})$, $3.29(\mathrm{~d}, J=15.6 \mathrm{~Hz}, 1 \mathrm{H}, \mathrm{ArCH}), 3.02$ (d, J=15.8 Hz, $\left.1 \mathrm{H}, \mathrm{ArCH}_{2}\right), 2.61$ (d, J=2.6 Hz, $\left.2 \mathrm{H}, \mathrm{C}_{2} \mathrm{CCH}_{2}\right), 2.03$ (t, $J=2.7 \mathrm{~Hz}, 1 \mathrm{H}, \mathrm{C} \equiv \mathrm{CH}), 1.59\left(\mathrm{~s}, 3 \mathrm{H}, \mathrm{CH}_{3}\right) .{ }^{13} \mathrm{C} \mathrm{NMR}\left(100 \mathrm{MHz}, \mathrm{CDCl}_{3}\right) \delta 159.3$ (aromatic C), 128.0 (aromatic CH), 126.3 (aromatic C), 125.0 (aromatic $\mathrm{CH}$ ), 120.3 (aromatic $\mathrm{CH}$ ), 109.6 (aromatic $\mathrm{CH}$ ), 86.8 (quaternary CO), 80.2 (alkynyl C), 70.5 (alkynyl CH), $40.6\left(\mathrm{CH}_{2}\right), 30.9\left(\mathrm{CH}_{2}\right), 25.8\left(\mathrm{CH}_{3}\right)$. IR v $3297(\mathrm{~m}), 3053$ (m), 2974 (m), 2931 (m), 2861 (m), 2651 (w), 2650 (w), 2562 (w), 2121 (w), 1691 (s), 1599 (m), 1576 (sh), 1482 (s), 1460 (s), 1409 (m), 1380 (m), 1325 (m), 1302 (sh), 1271 (sh), 1240 (s), 1186 (w), 1144 (w), 1116 (w), $1070(\mathrm{~m}), 1017(\mathrm{w}), 957(\mathrm{w}), 920(\mathrm{sh}), 886(\mathrm{~s}), 859(\mathrm{sh}), 786(\mathrm{~m}), 749(\mathrm{~s}), 711(\mathrm{~m}), 649(\mathrm{~s})$. MS (EI) found for $\mathrm{C}_{12} \mathrm{H}_{12} \mathrm{O}^{+}(\mathrm{M})$ 172. DEPT experiments (see spectra) was not conclusive to differentiate definitively the two acetylene carbons at 80.2 and $70.5 \mathrm{ppm}$, as even the quaternary carbon displayed a significant signal in all DEPT experiments. To confirm further the assignment, 62 was deprotonated with ${ }^{n} \mathrm{BuLi}$ in THF and the 
resulting organolithium was quenched with $\mathrm{D}_{2} \mathrm{O}$. The obtained C-D coupling was much larger at 70.2 ppm (39 $\mathrm{Hz}$ vs $7.7 \mathrm{~Hz}$ ), confirming definitively this carbon to be the terminal one: ${ }^{1} \mathrm{H} \mathrm{NMR}\left(400 \mathrm{MHz}, \mathrm{CDCl}_{3}\right) \oint 7.16$ $(\mathrm{d}, J=8.2 \mathrm{~Hz}, 1 \mathrm{H}, \mathrm{Ar} H), 7.12(\mathrm{~d}, J=7.7 \mathrm{~Hz}, 1 \mathrm{H}, \mathrm{Ar} H), 6.86(\mathrm{t}, J=7.4 \mathrm{~Hz}, 1 \mathrm{H}, \mathrm{Ar} H), 6.78(\mathrm{~d}, J=8.1 \mathrm{~Hz}, 1$ $\mathrm{H}, \operatorname{Ar} H), 3.30\left(\mathrm{~d}, J=15.8 \mathrm{~Hz}, 1 \mathrm{H}, \mathrm{ArCH}_{2}\right), 3.03\left(\mathrm{~d}, J=15.8 \mathrm{~Hz}, 1 \mathrm{H}, \mathrm{ArCH}_{2}\right), 2.62\left(\mathrm{~s}, 2 \mathrm{H}, \mathrm{C} \equiv \mathrm{CCH}_{2}\right), 1.61(\mathrm{~s}$, $\left.3 \mathrm{H}, \mathrm{CH}_{3}\right) .{ }^{13} \mathrm{C} \mathrm{NMR}\left(100 \mathrm{MHz}, \mathrm{CDCl}_{3}\right) \delta .158 .5,128.0,126.4,125.1,120.3,109.6,86.9,79.7\left(\mathrm{t},{ }^{2} J(\mathrm{C}, \mathrm{D})=7.7\right.$ $\mathrm{Hz}, C \equiv \mathrm{CD}), 70.2\left(\mathrm{t},{ }^{1} J(\mathrm{C}, \mathrm{D})=39 \mathrm{~Hz}, \mathrm{C} \equiv C \mathrm{D}\right), 40.7,30.9,25.7$.

\section{Labeled 2-methyl-2-(prop-2-ynyl)-2,3-dihydrobenzofuran (62')}

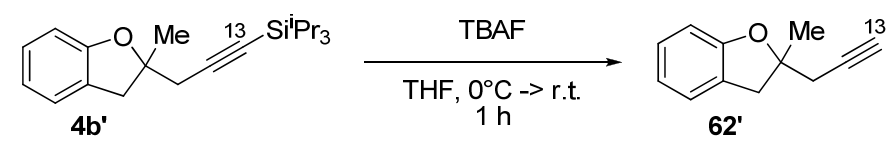

The same procedure as for compounds 62 was used to prepare the ${ }^{13} \mathrm{C}$ labeled compound 62 '. ${ }^{13} \mathrm{C}$ labeled triisopropyl-[3-(2-methyl-2,3-dihydro-benzofuran-2-yl)-prop-1-ynyl]-silane (4b') (51 mg, 0.15 mmol, 1.0 equiv) was dissolved in THF $(0.7 \mathrm{~mL})$ and the resulting solution was stirred at $0{ }^{\circ} \mathrm{C}$ for $5 \mathrm{~min}$. TBAF $(1 \mathrm{M}$ solution in THF, $0.3 \mathrm{~mL}, 0.3 \mathrm{mmol}, 2.0$ equiv) was then added and the mixture was stirred a $0{ }^{\circ} \mathrm{C}$ for $50 \mathrm{~min}$. The reaction mixture was allowed to warm to room temperature and stirred for $10 \mathrm{~min}$. $\mathrm{NH}_{4} \mathrm{Cl}(\mathrm{saturated}$ solution, $12 \mathrm{~mL})$ was added followed by water $(4 \mathrm{~mL})$. The aqueous phase was extracted with $\mathrm{Et}_{2} \mathrm{O}(3 \mathrm{x} 5 \mathrm{~mL})$ and the combined organic layers were washed with $\mathrm{NH}_{4} \mathrm{Cl}$ (saturated solution, $12 \mathrm{~mL}$ ) and brine $(12 \mathrm{~mL})$, dried over $\mathrm{MgSO}_{4}$, filtered and concentrated under reduced pressure. Purification by column chromatography $\left(\mathrm{SiO}_{2}\right.$, PET/EtOAc 99:1) afforded ${ }^{13} \mathrm{C}$ labeled 2-methyl-2-(prop-2-ynyl)-2,3-dihydrobenzofuran (62') (19 mg, 0.11 mmol, 71\% yield) as a colorless oil. $\left.{ }^{13} \mathrm{C} \mathrm{NMR} \mathrm{(100} \mathrm{MHz}, \mathrm{CDCl}_{3}\right) \oint 159.3$ (aromatic C), 128.0 (aromatic $\mathrm{CH}$ ), 126.3 (aromatic C), 125.0 (aromatic CH), 120.3 (aromatic CH), 109.6 (aromatic CH), 86.8 (alkynyl C), 80.2 (quaternary C), $70.5(10 x$ more intensive, alkynyl $\mathrm{CH}), 40.6\left(\mathrm{CH}_{2}\right), 30.9\left(\mathrm{CH}_{2}\right), 25.8\left(\mathrm{CH}_{3}\right)$.

\section{Spectra of New Compounds.}


solvent: $<\mathrm{CDCl} 3>$

Frequency $400,13 \mathrm{MHz}$<smiles>C=C(C)Cc1cc(Br)ccc1O</smiles>

1c

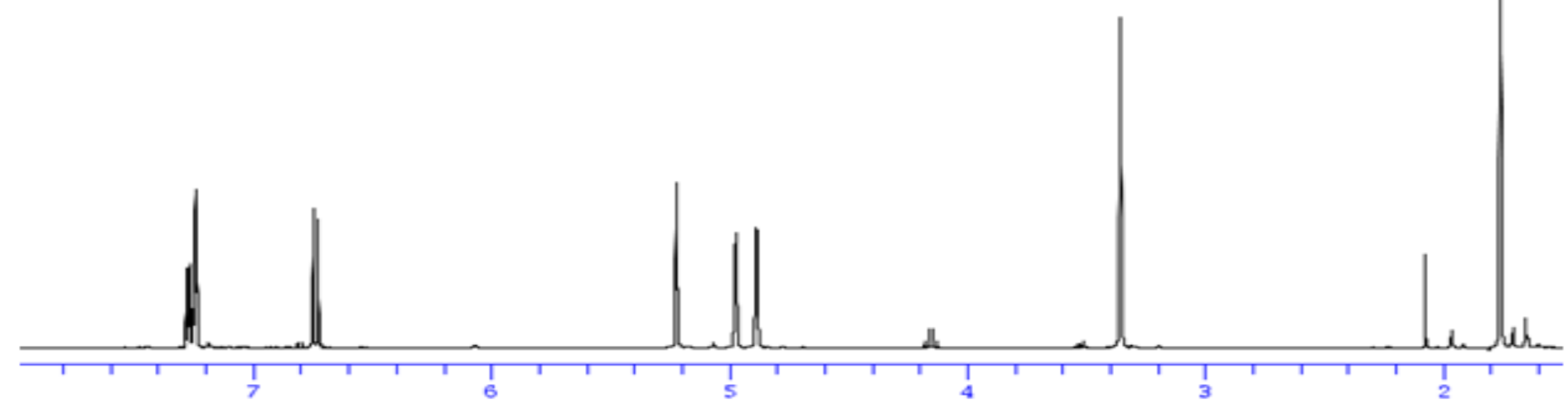

solvent: $<\mathrm{CDCI} 3>$

Frequency. $400.13 \mathrm{MHz}$
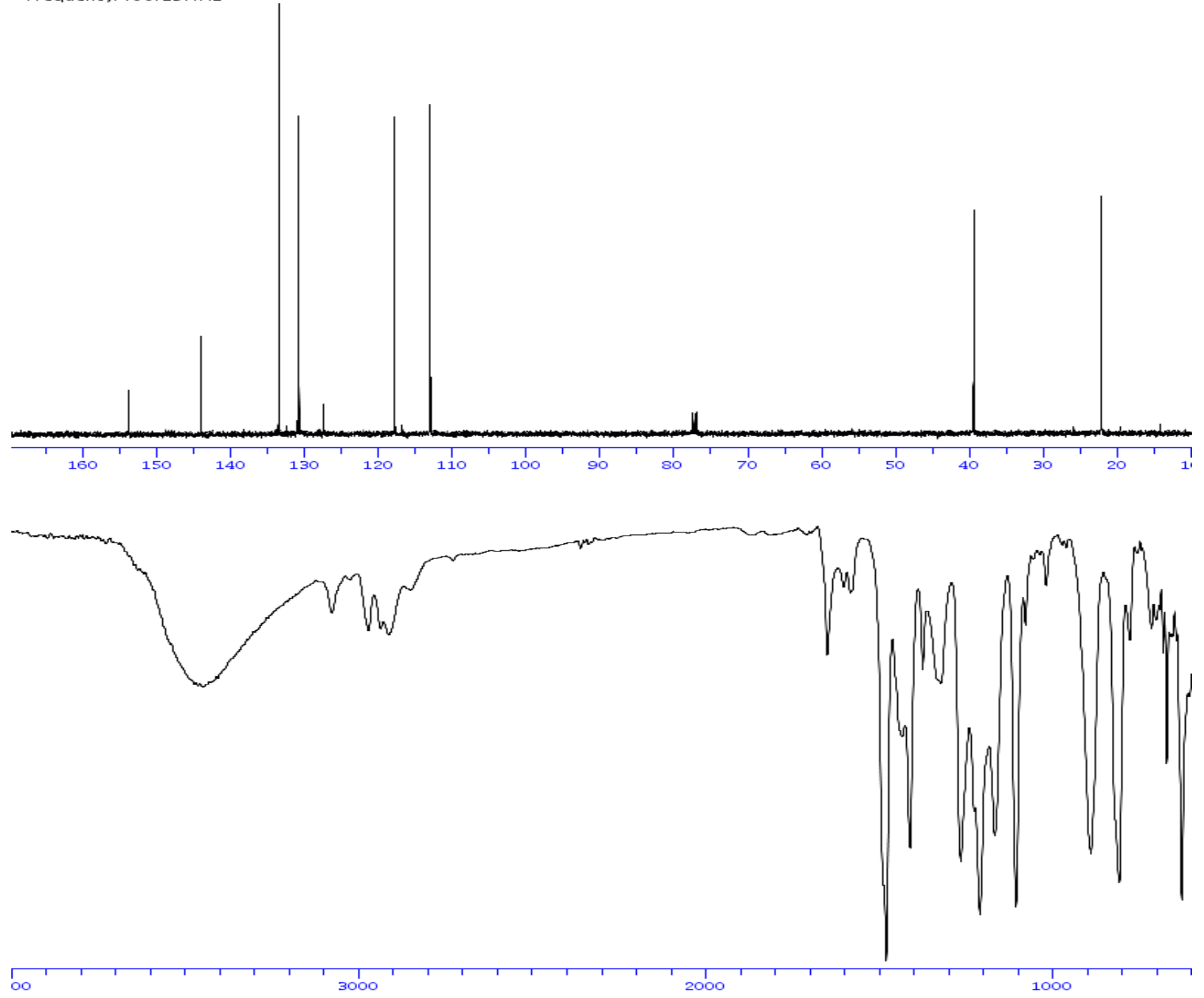
solvent: $\langle\mathrm{CDCI}>$

Frequency. $100.61 \mathrm{MHz}$<smiles>C=C(C)Cc1cc([N+](=O)[O-])ccc1O</smiles>

$1 e$
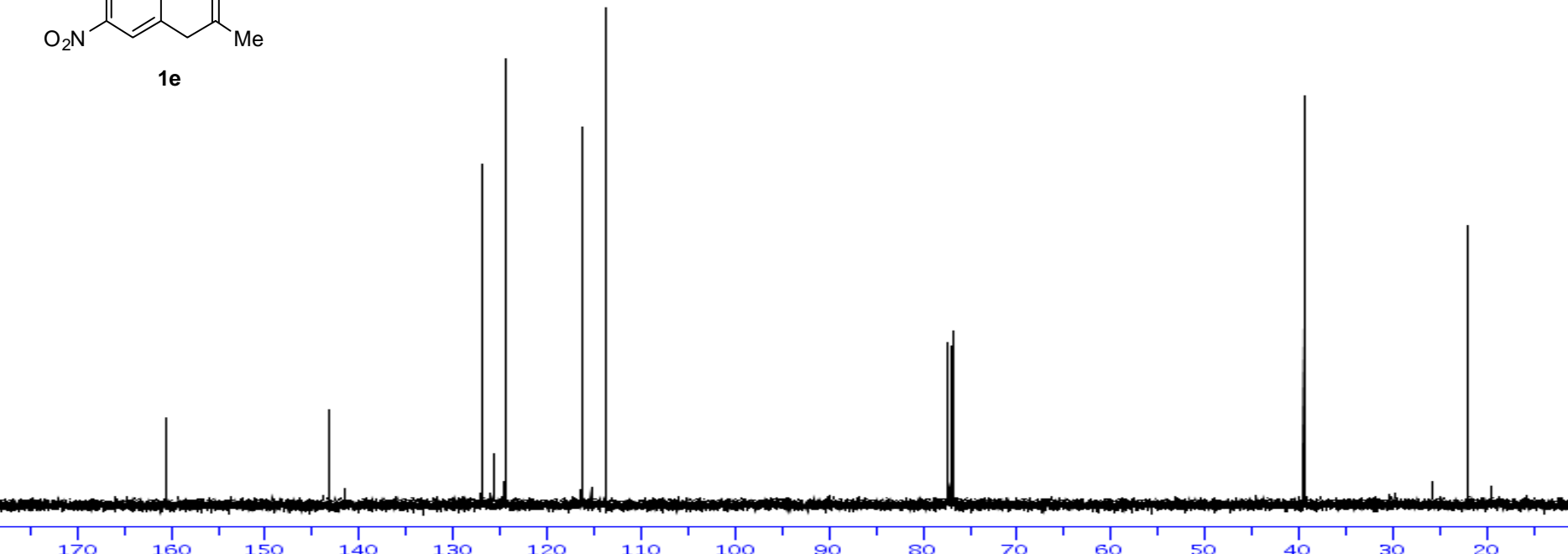

solvent: $\langle\mathrm{CDCI} 3\rangle$

Frequency. $100.61 \mathrm{MHz}$
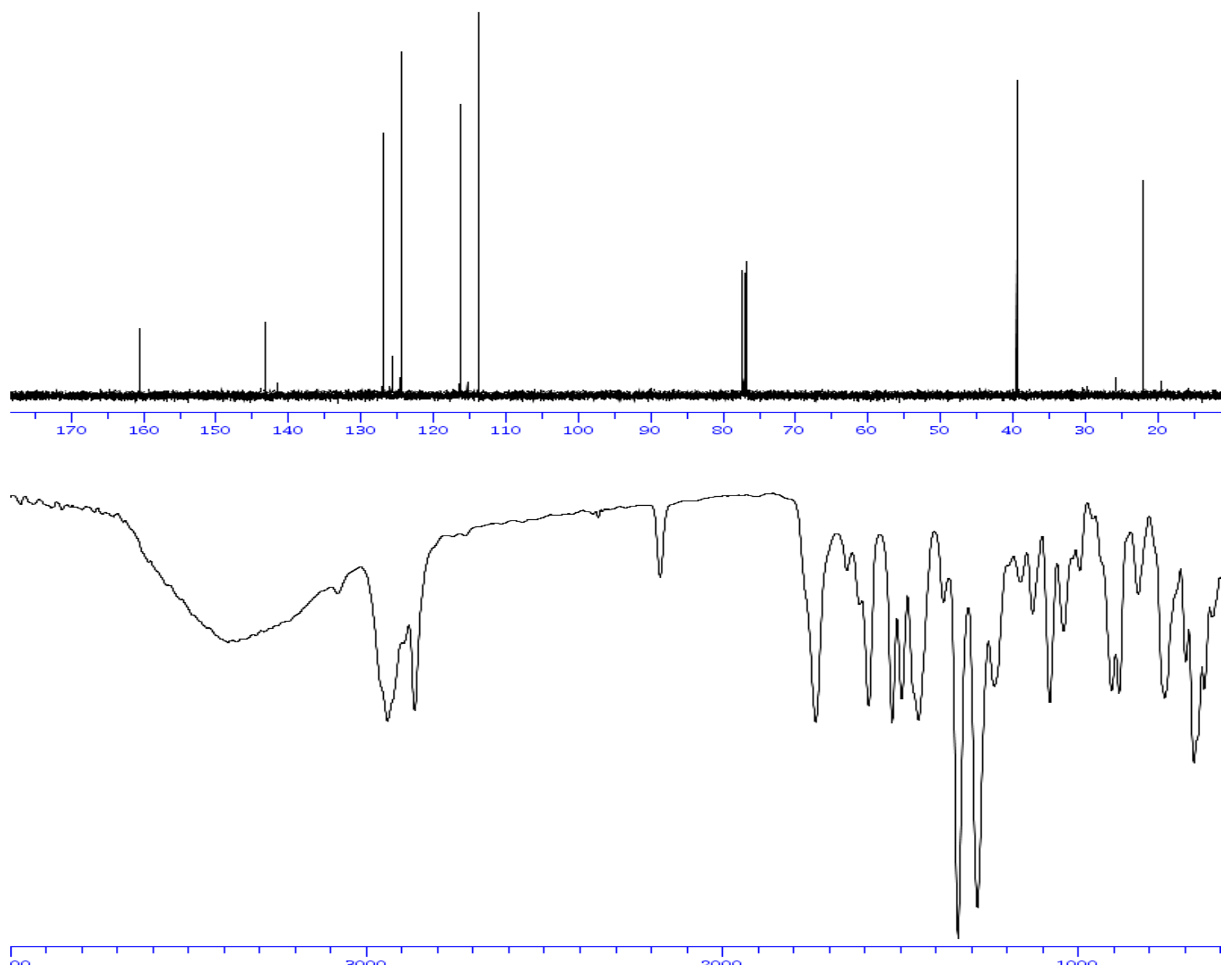
<smiles>C=C(C)Cc1cc(C(C)=O)ccc1O</smiles>

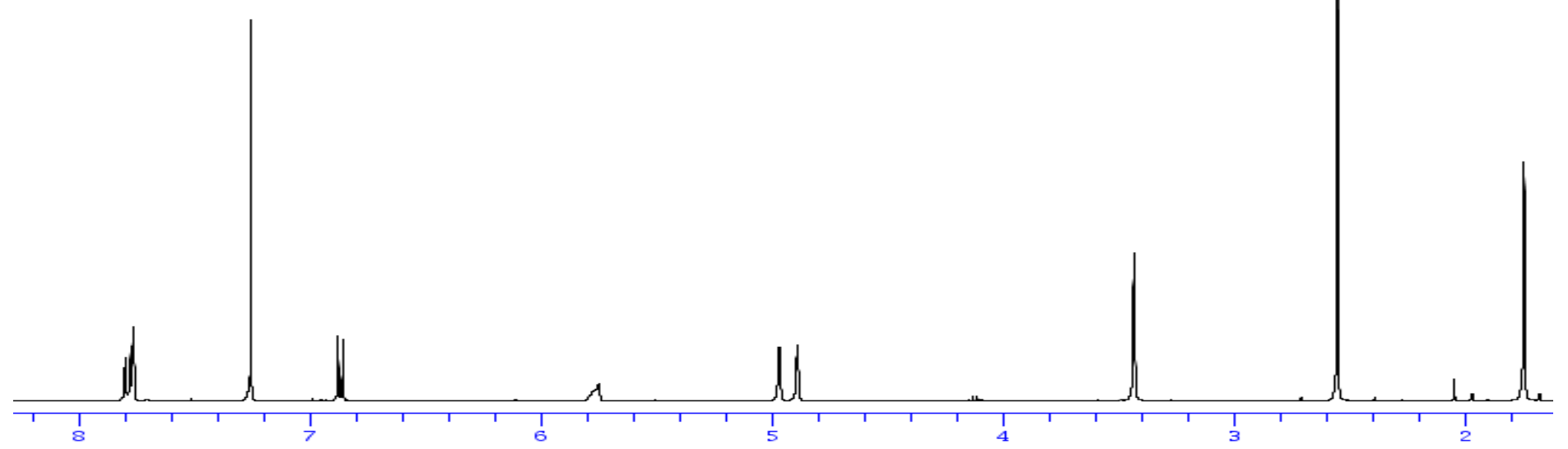

solvent: $<\mathrm{CDCI} 3\rangle$
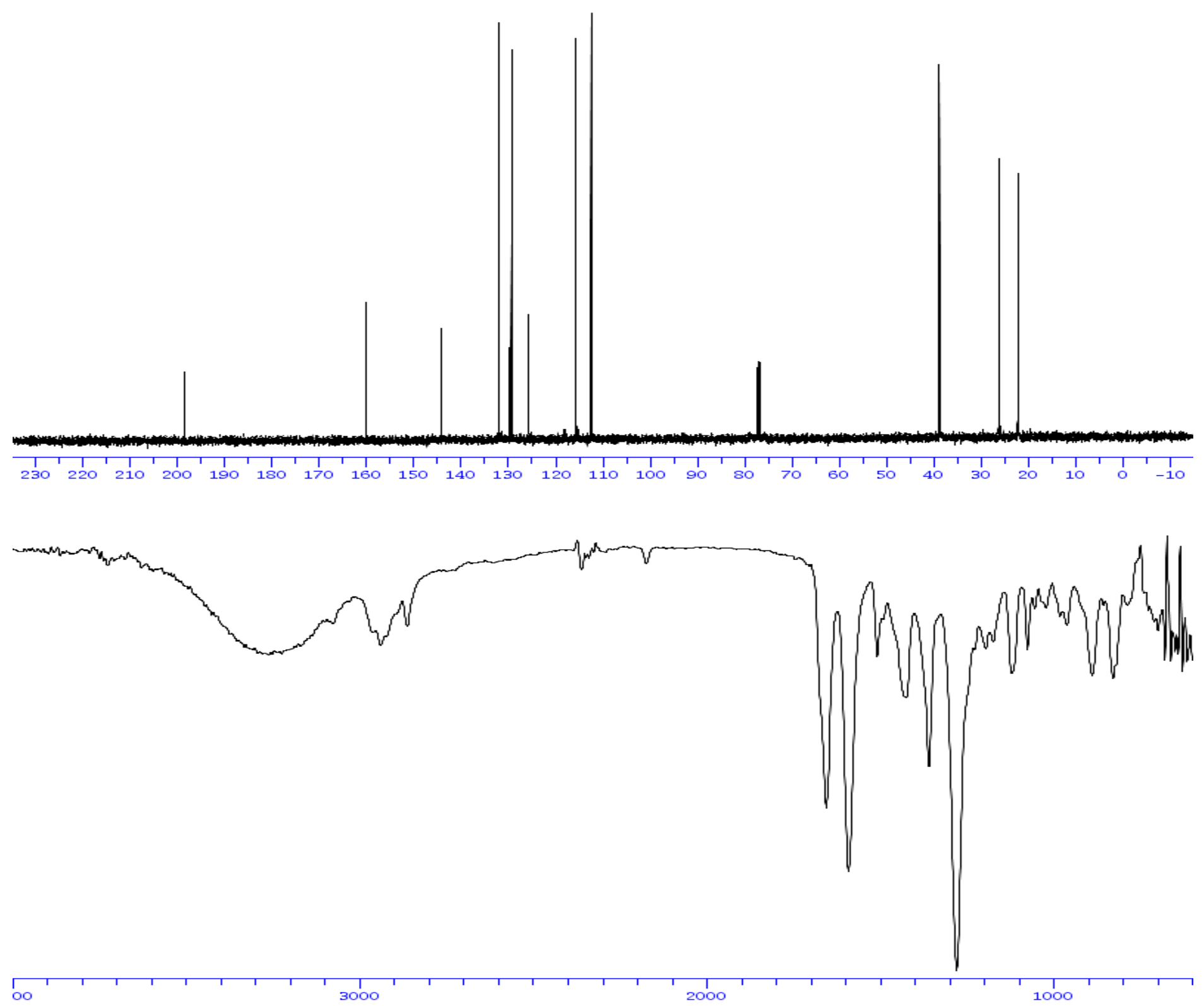
solvent: $<\mathrm{CDCI} 3>$

Frequency. 400. 13 $\mathrm{m} \mathrm{MHz}$

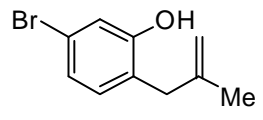

$1 \mathrm{~g}$

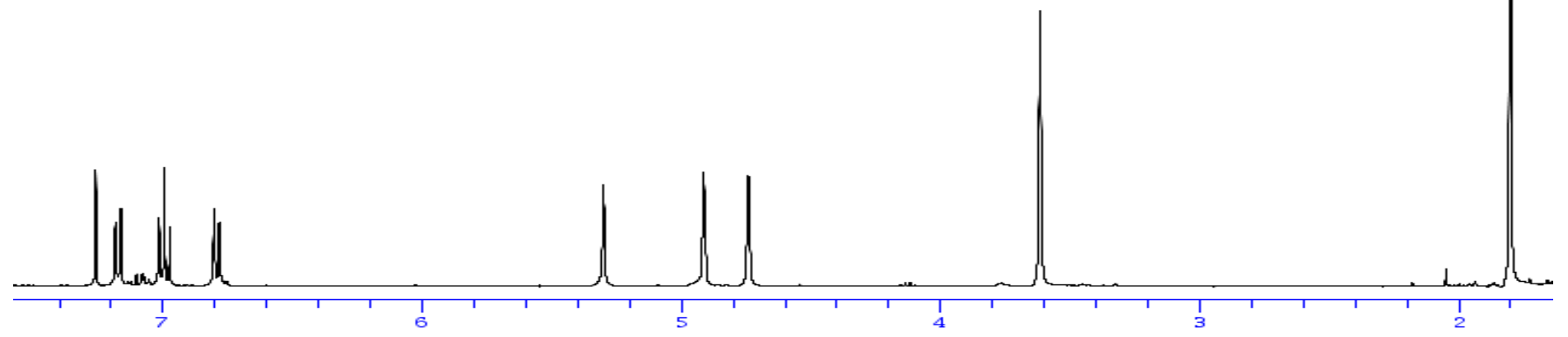

Solvent: <CDCI3 >

Frequency. $100.61 \mathrm{MHz}$
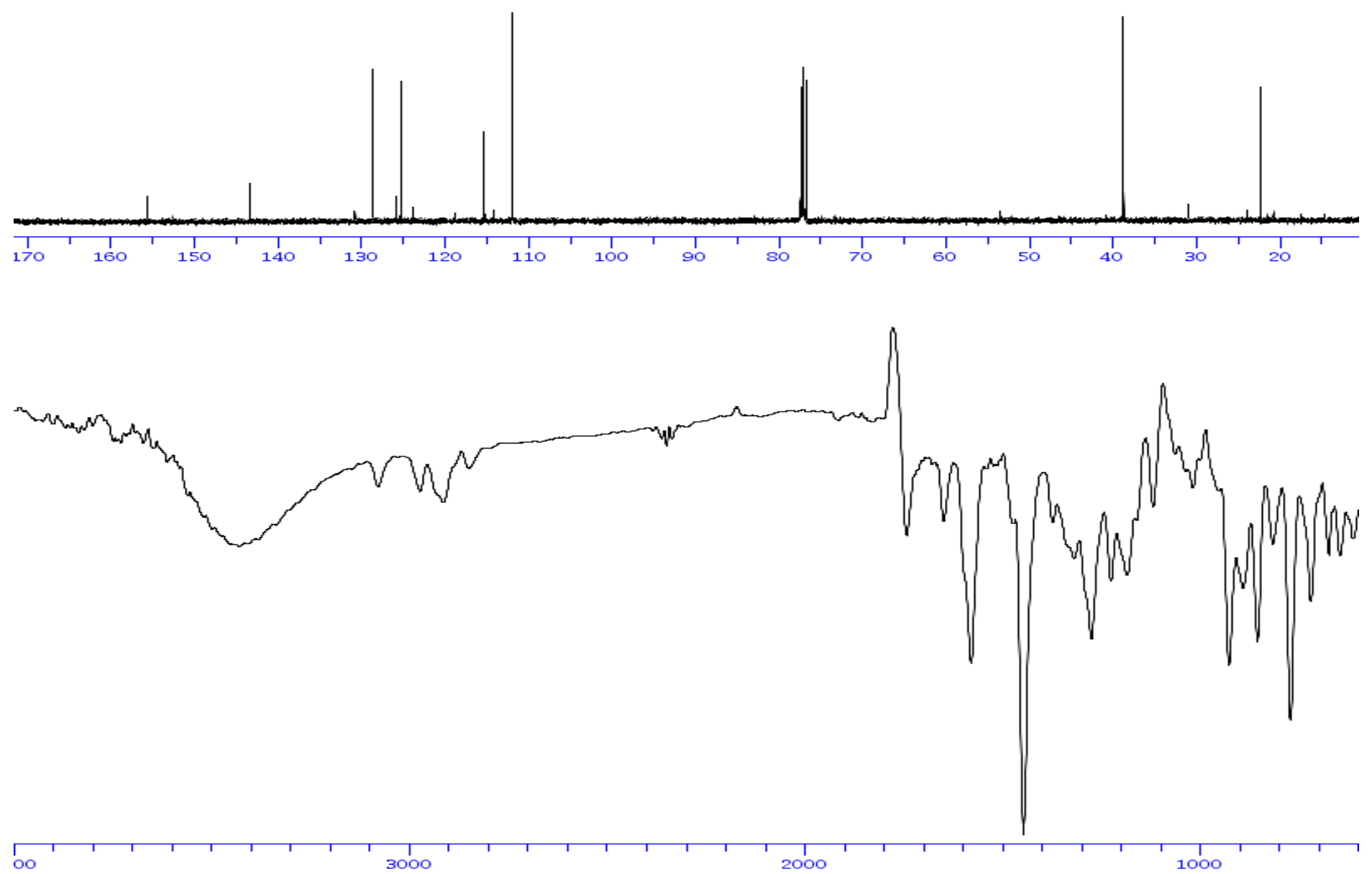
solvent: <CDCI3 >

Frequency. 400.13 $\mathrm{MHz}$
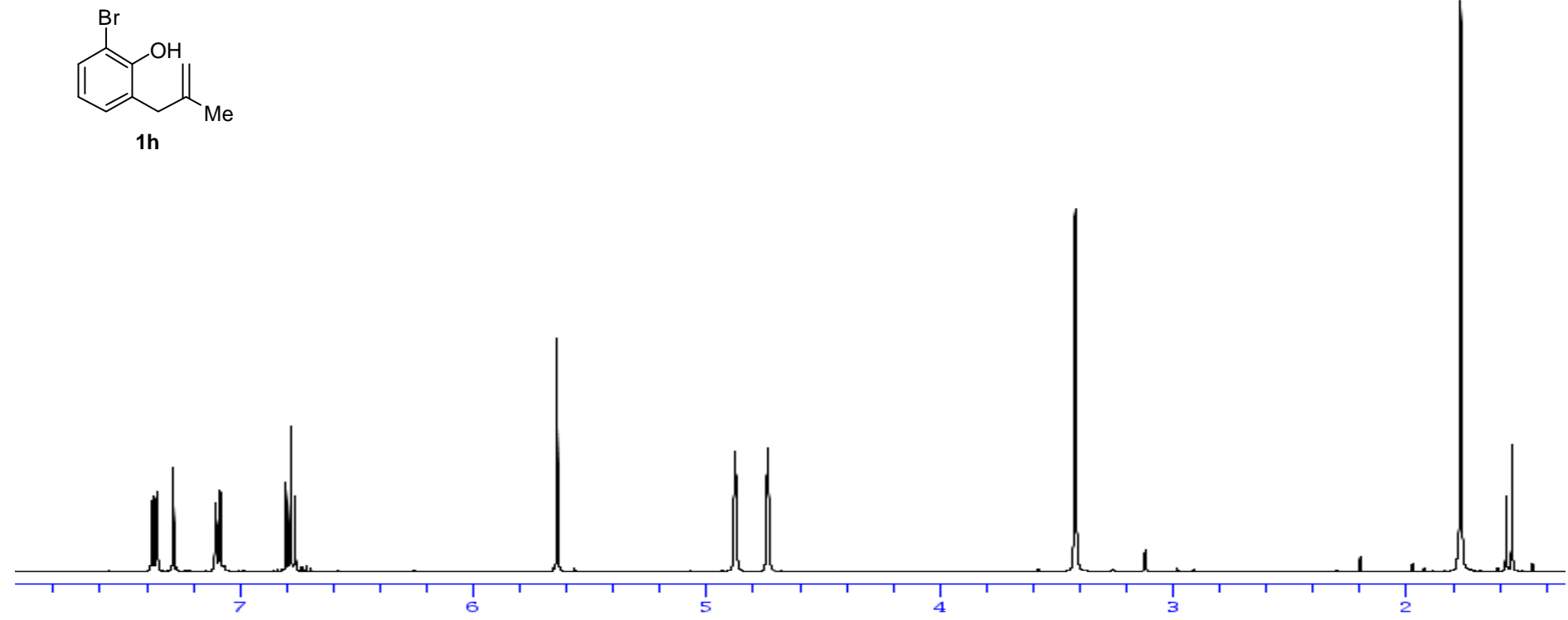

solvent: $\langle\mathrm{CDCI} 3\rangle$

Frequency. $100.61 \mathrm{MHz}$
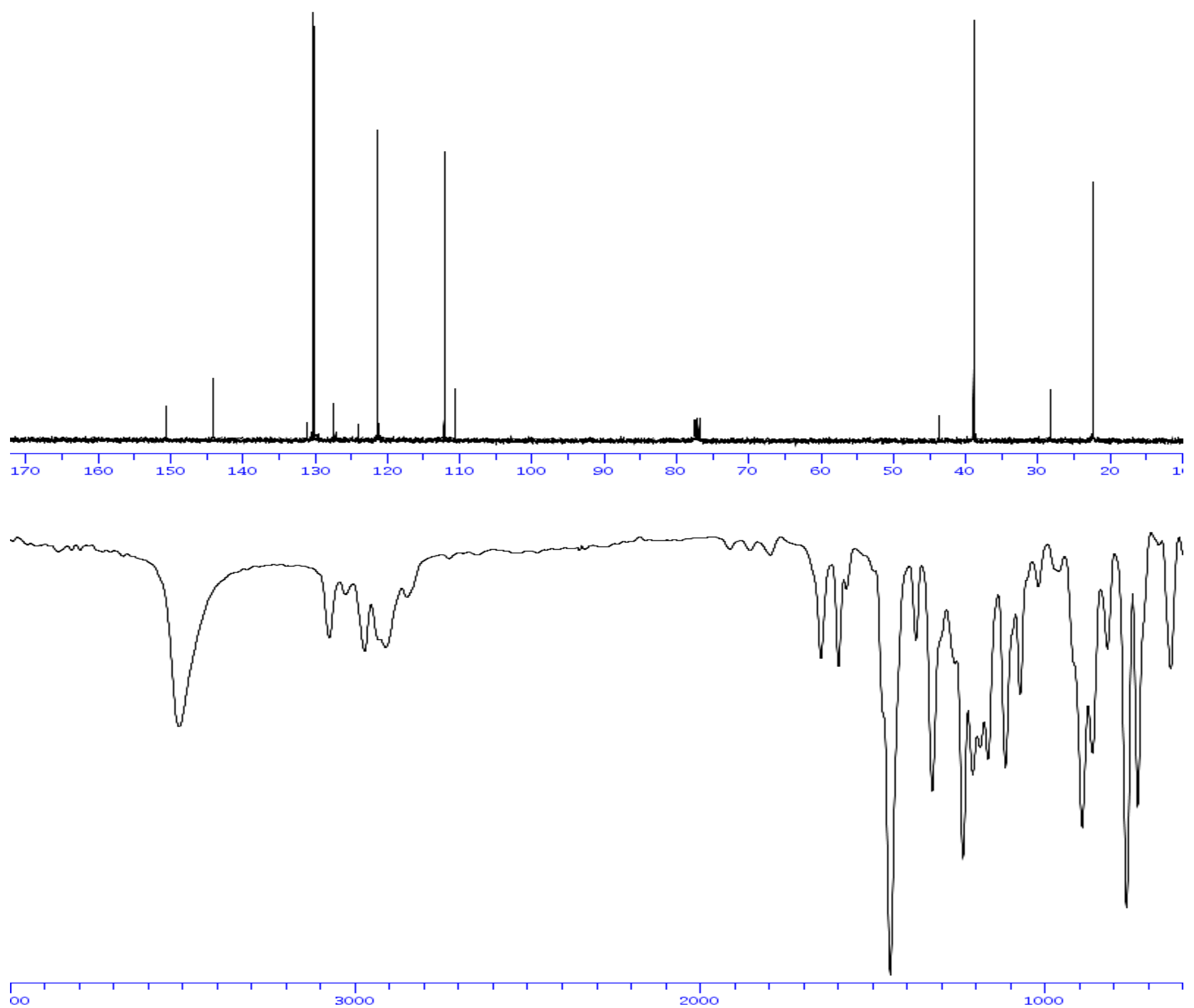


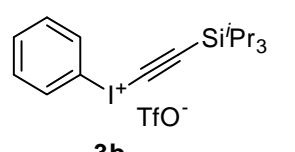

$3 b$

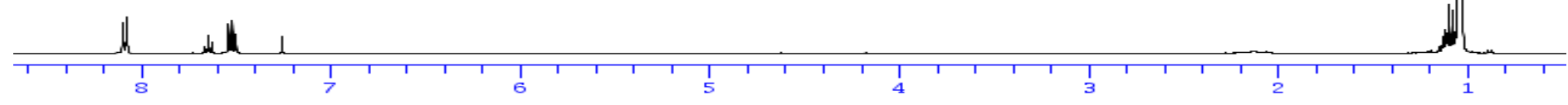

solvent: $<\mathrm{CDCI} 3>$

Frequency. $100.61 \mathrm{MHz}$
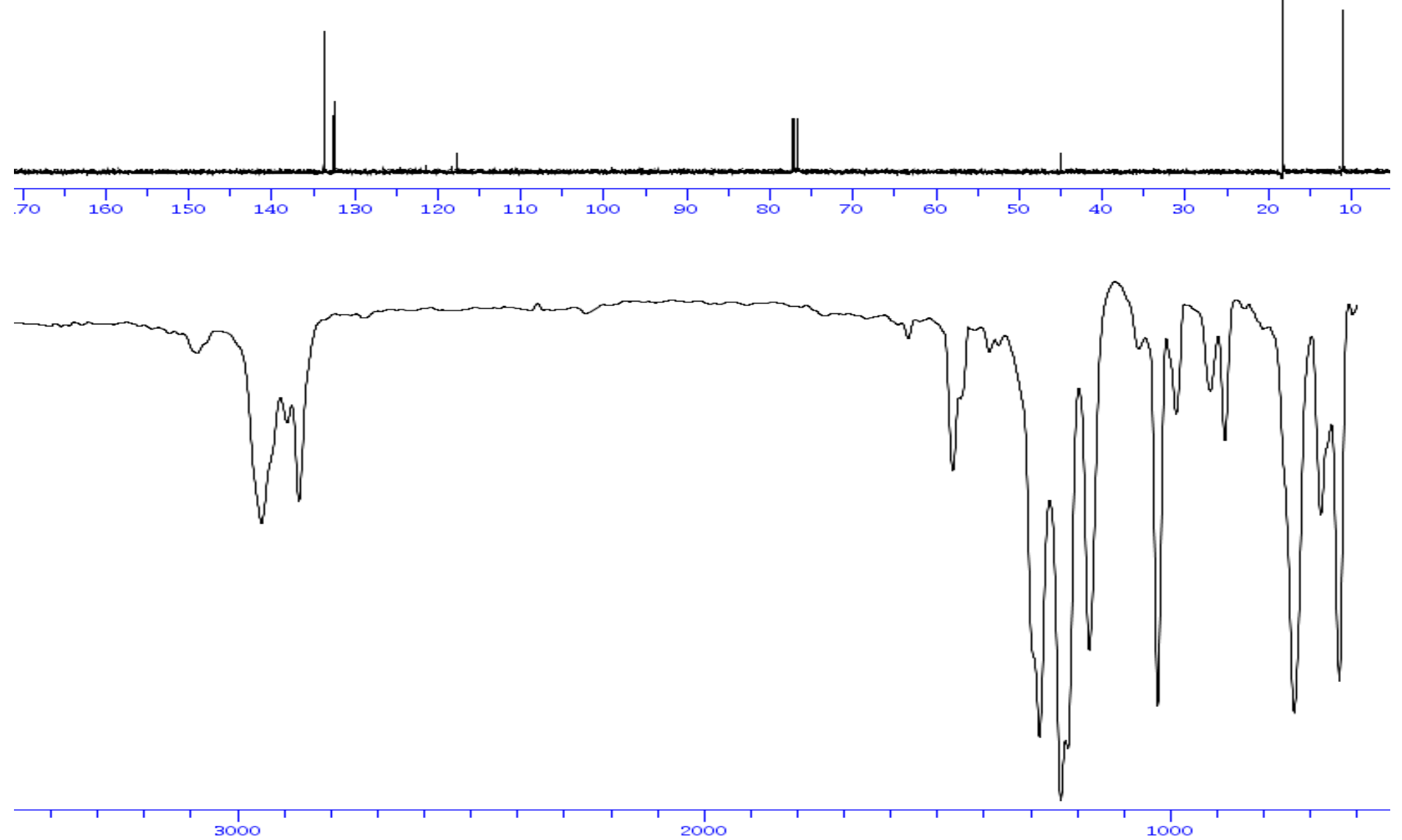

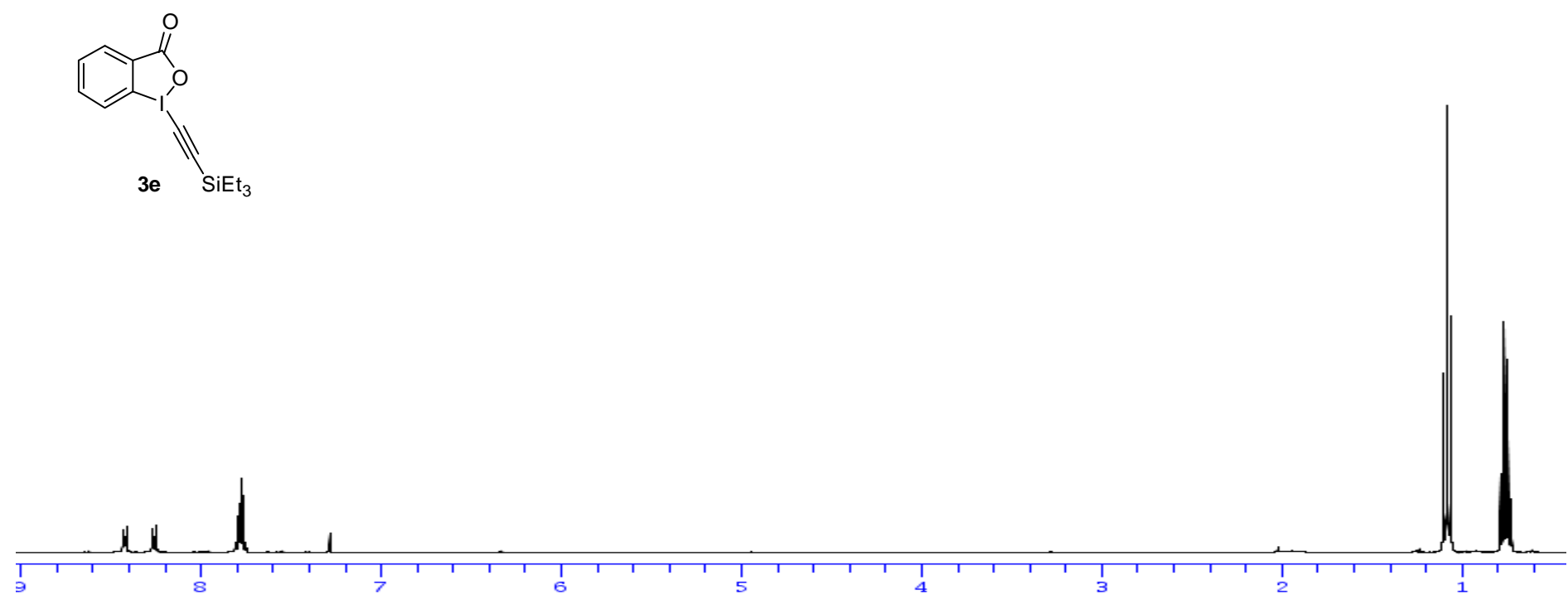

solvent: $\langle\mathrm{CDCI}\rangle$

Frequency. 400.13 $\mathrm{MHz}$
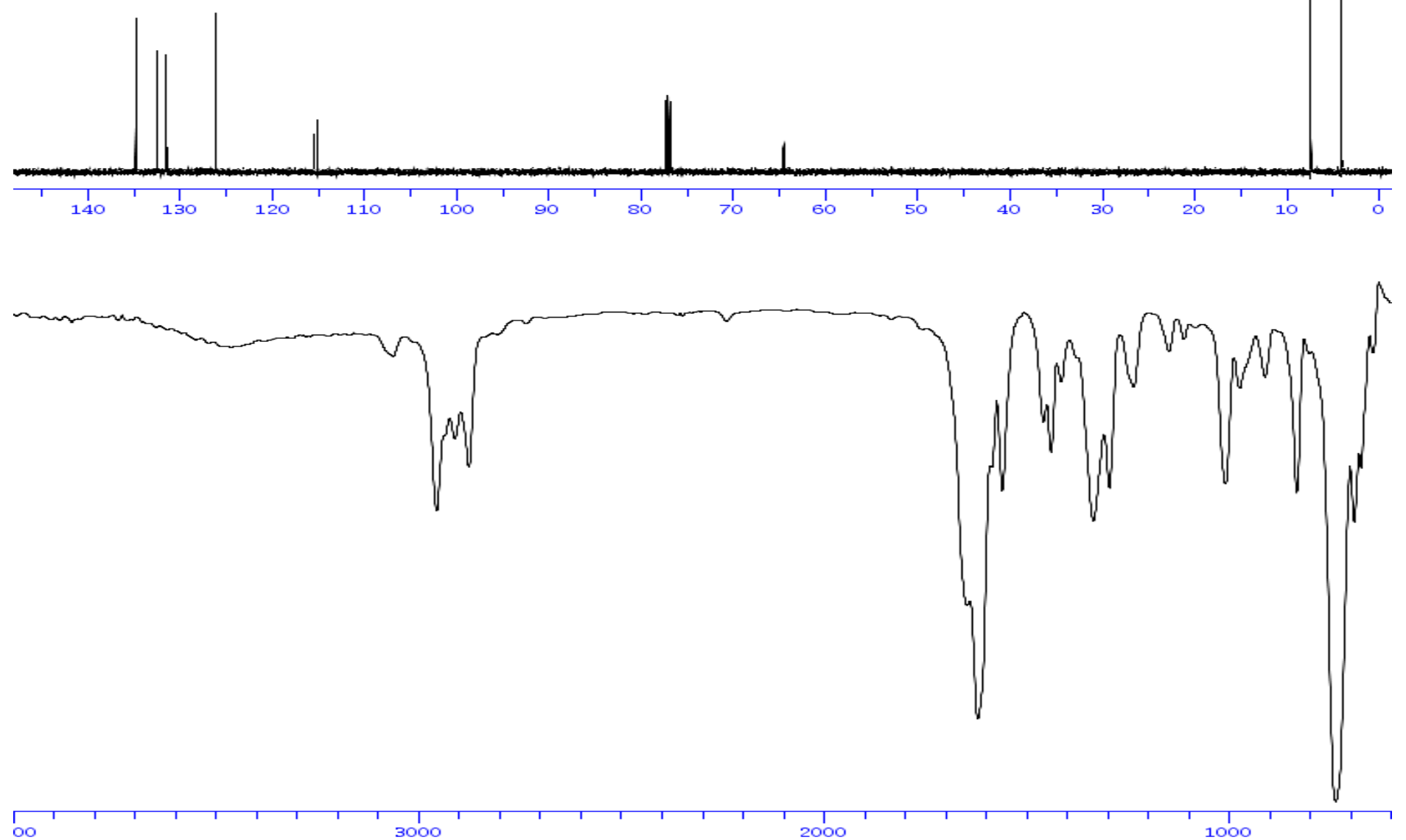
solvent: $<\mathrm{CDC1} 3>$

Frequency $400.13 \mathrm{MHz}$

$\sim^{\mathrm{Me}} \mathrm{C}^{\mathrm{Si}^{\mathrm{i}} \mathrm{Pr}_{3}}$

4b

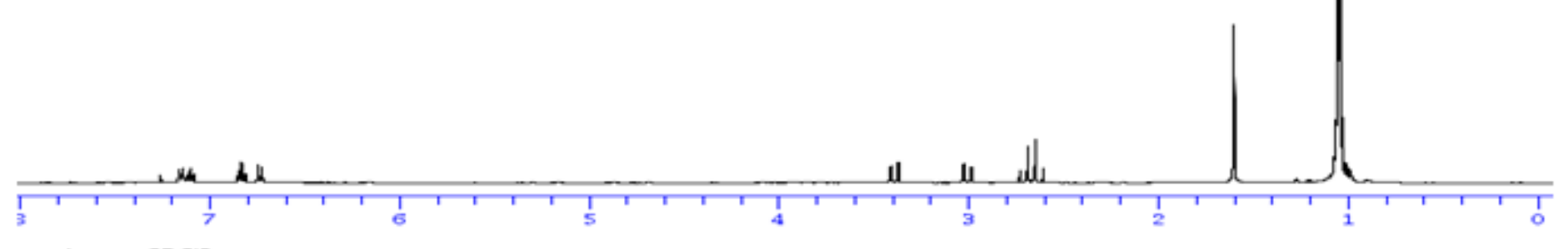

solvent: <CDCI3 >

Frequency 100.61MHz
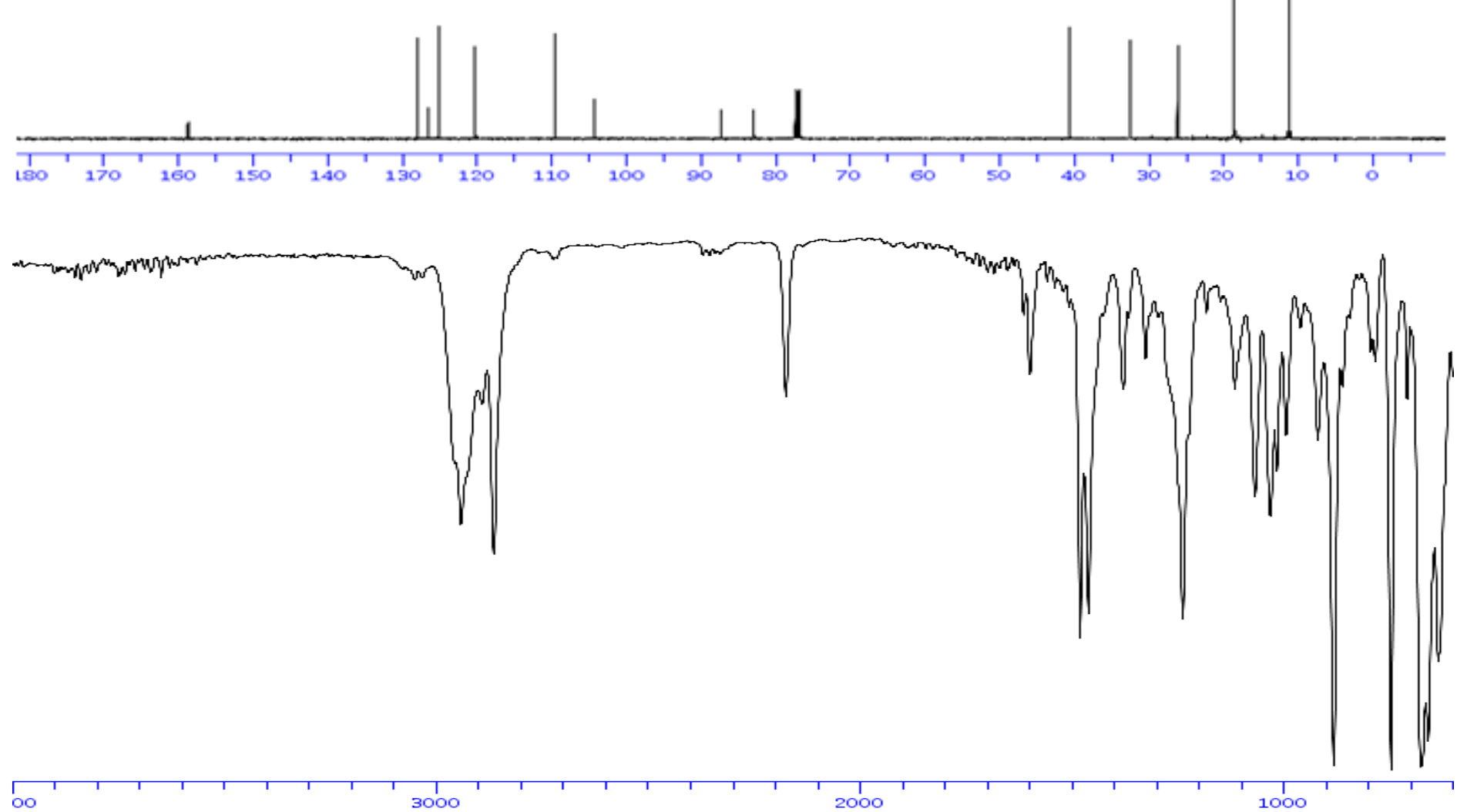


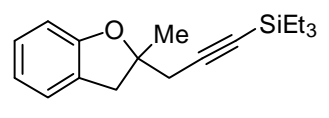

$4 c$

\section{so}
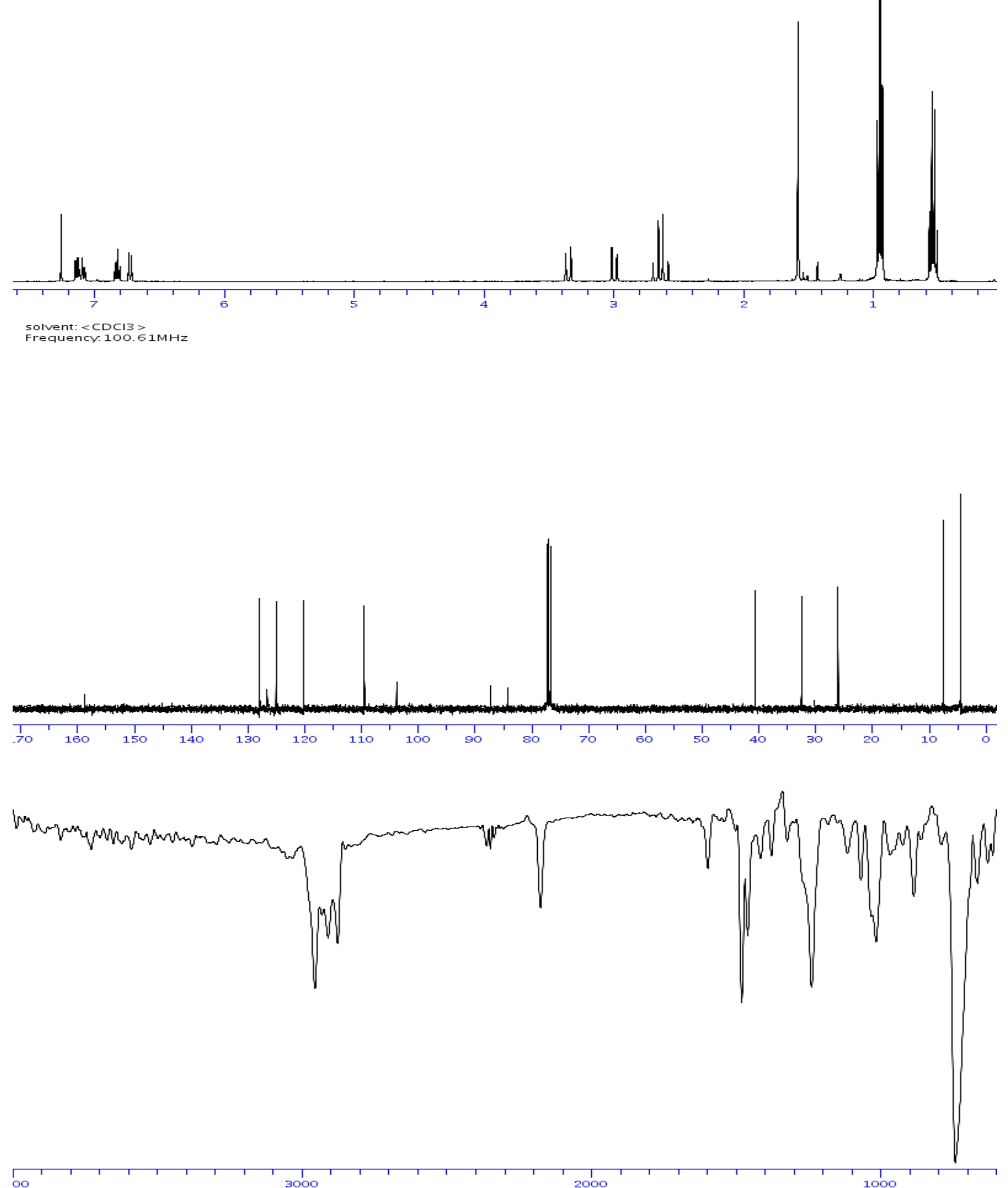


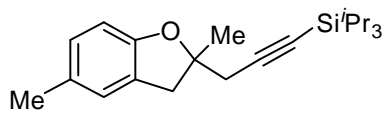

4d

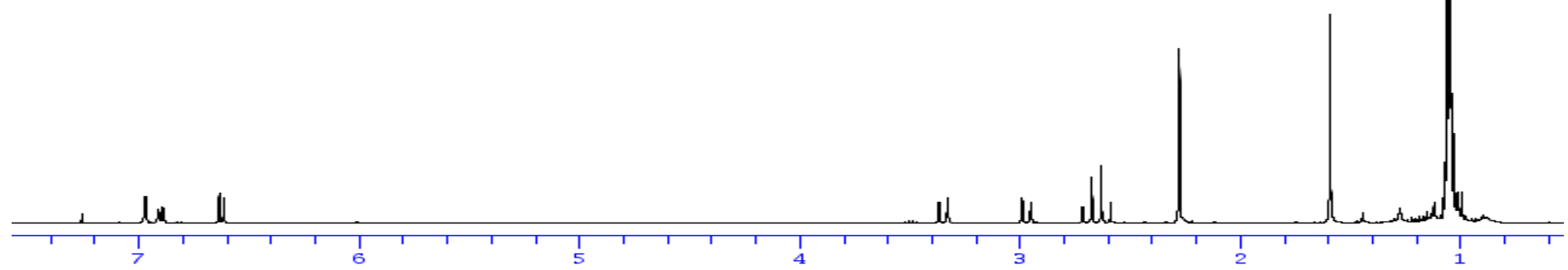

Solvent: $<\mathrm{CDCI} 3\rangle$
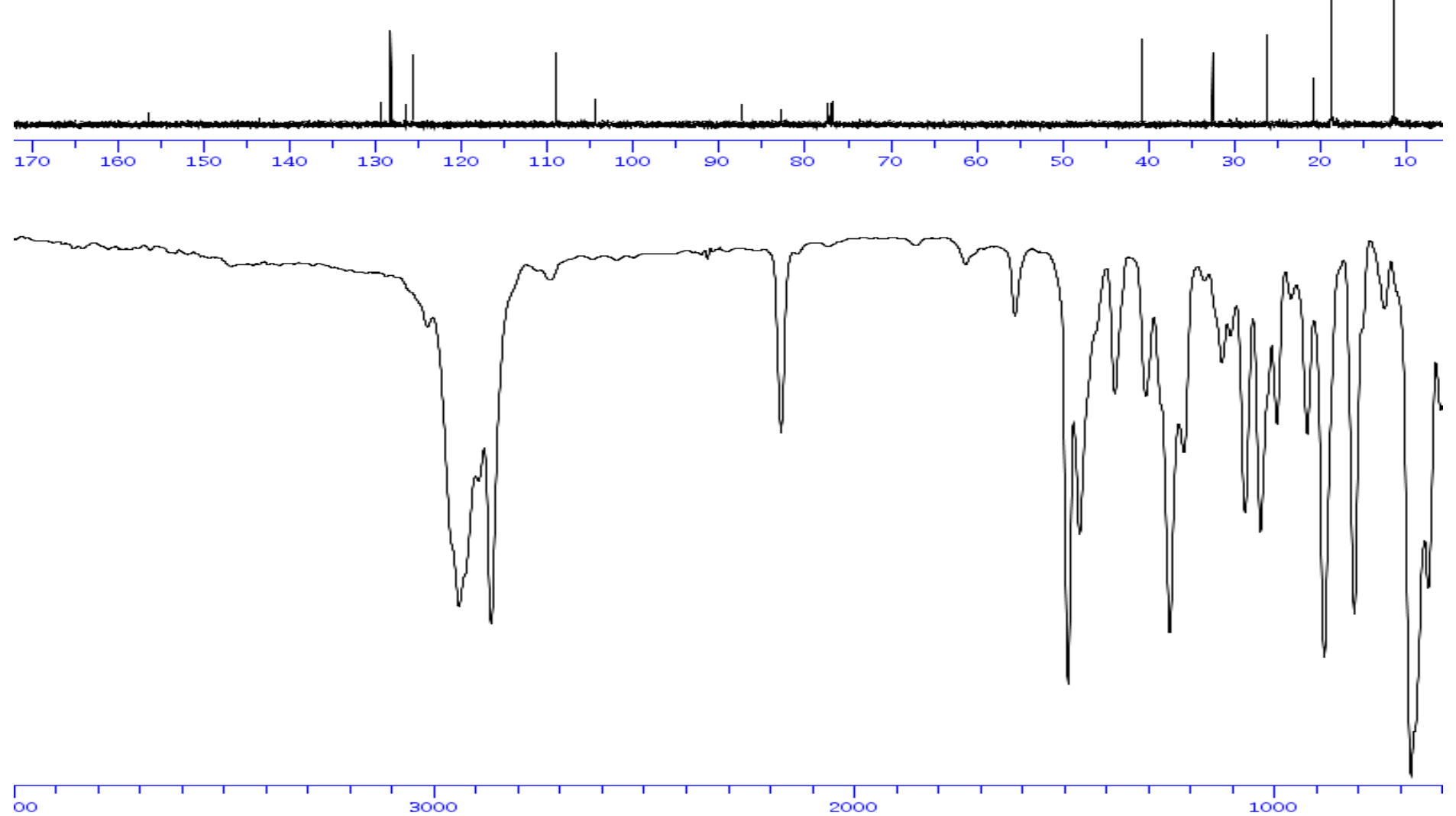
solvent: $<C D C I 3>$
Frequency. $400.13 \mathrm{MHz}$

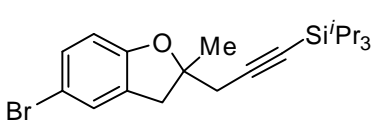

$4 \mathrm{e}$

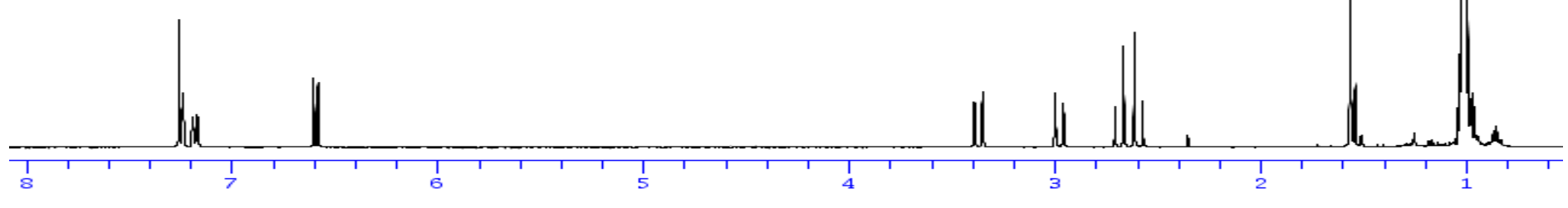

solvent: $<$ CDCL3 $>$
Frequency. $100.61 \mathrm{MHz}$
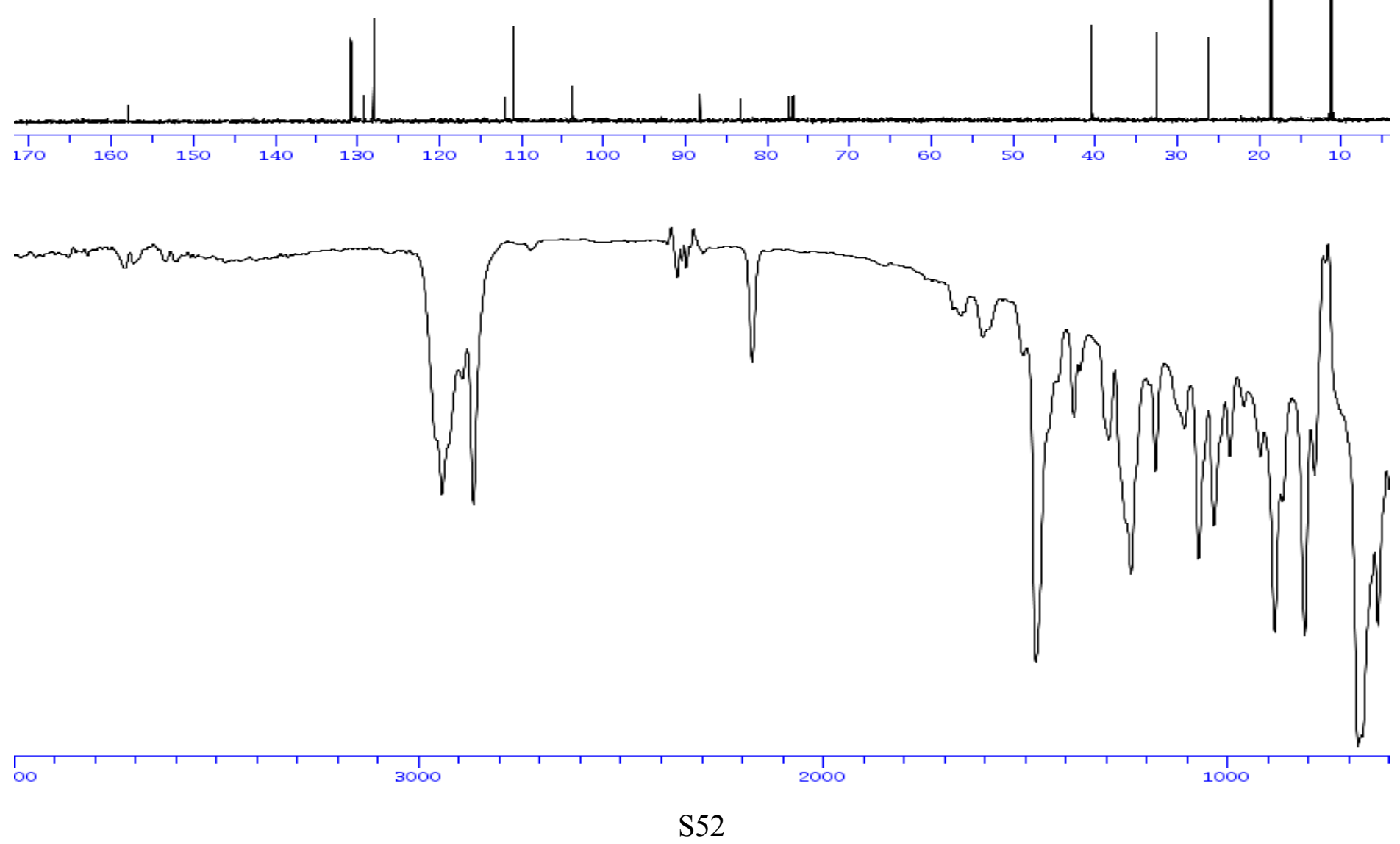
solvent: $<C D C I 3>$
Frequency. $400.13 \mathrm{MHz}$

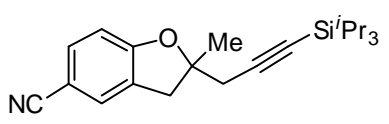

$4 f$

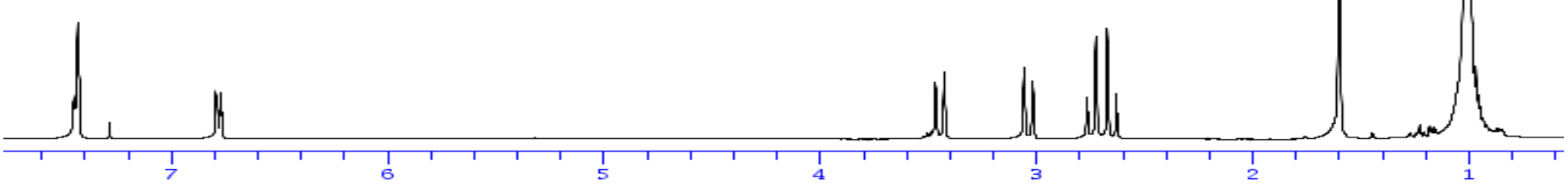

Solvent: $<C D C I 3>$
Frequency. $100.61 \mathrm{MHz}$
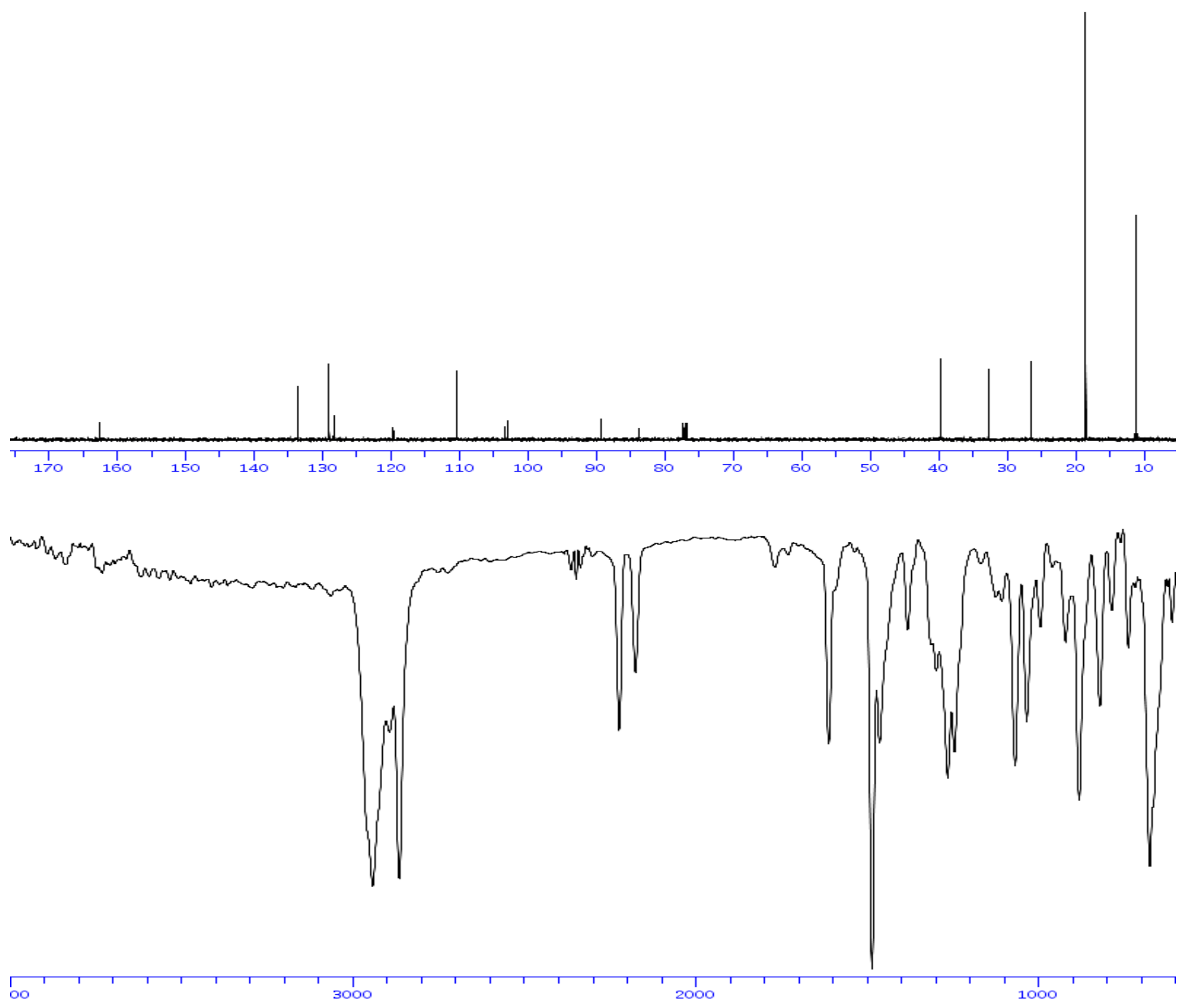

S53 


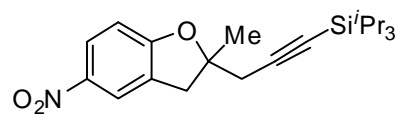

$4 \mathrm{~g}$

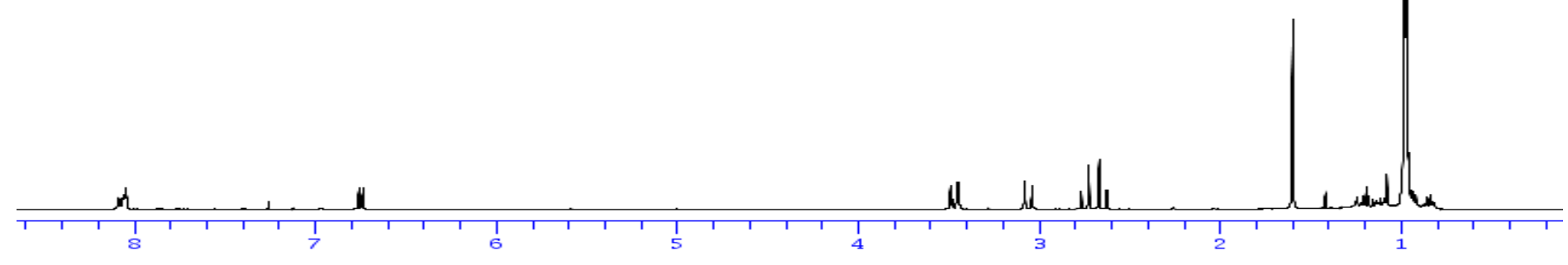

solvent: $<C D C L 3>$

Frequency. $100.61 \mathrm{MHz}$
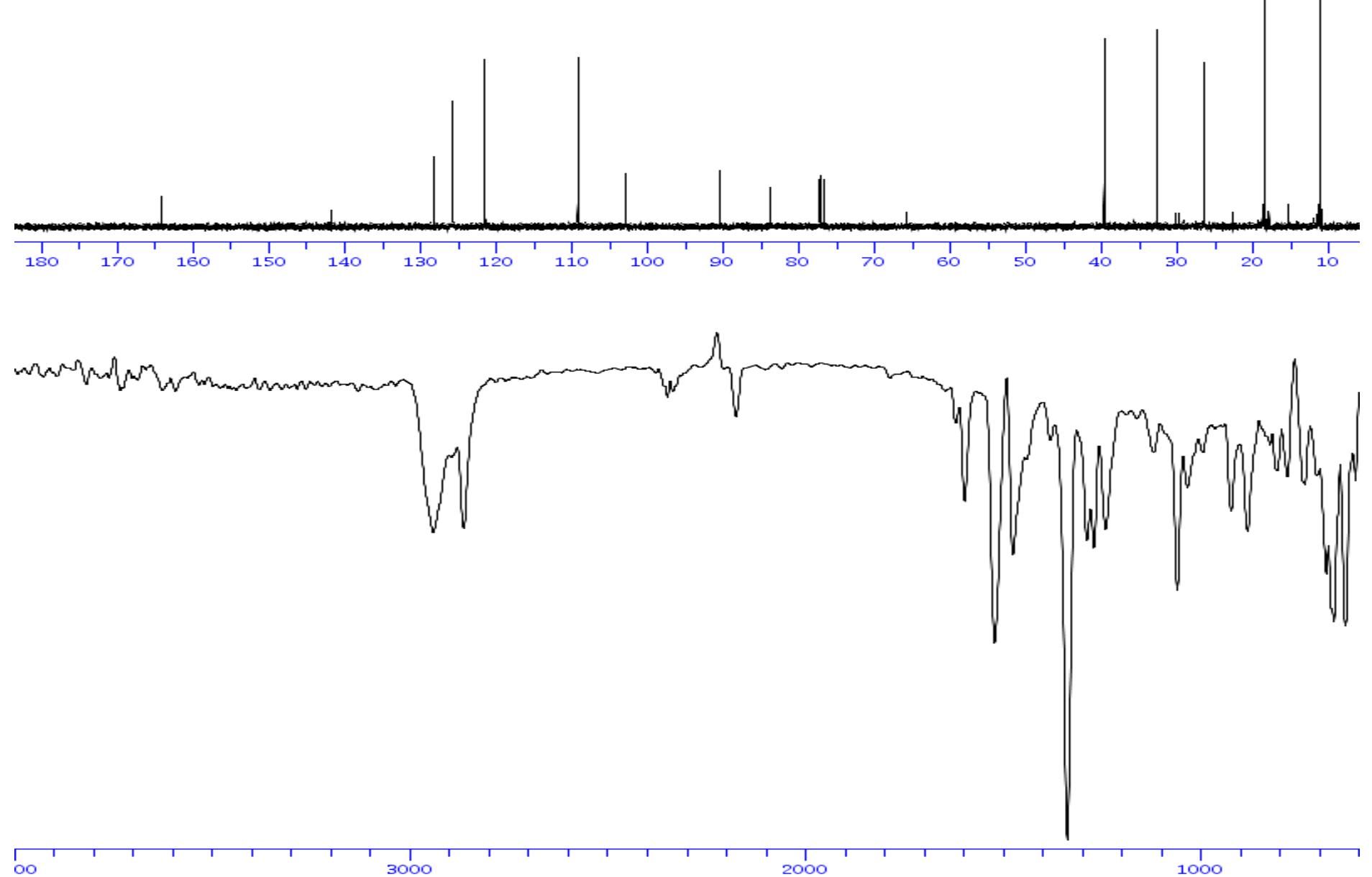

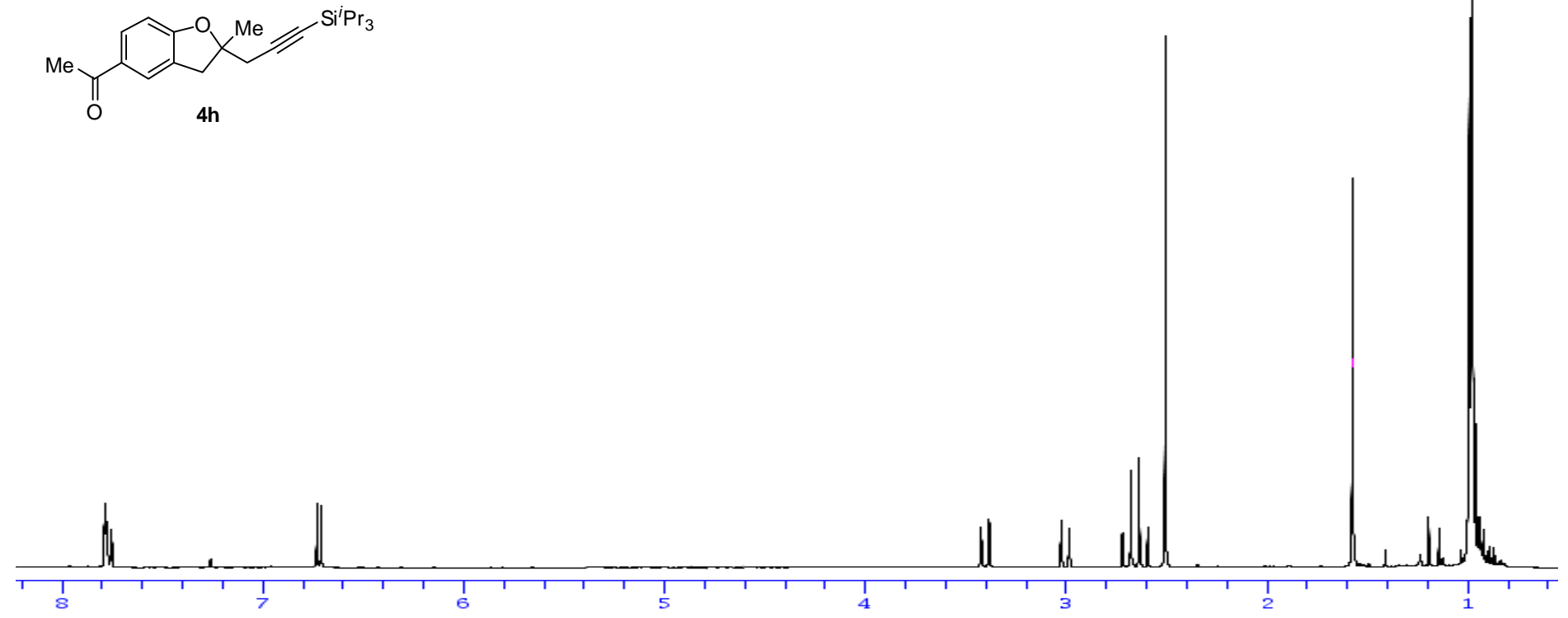

solvent: $\langle\mathrm{CDCI} 3\rangle$

Frequency. 100.61M Hz
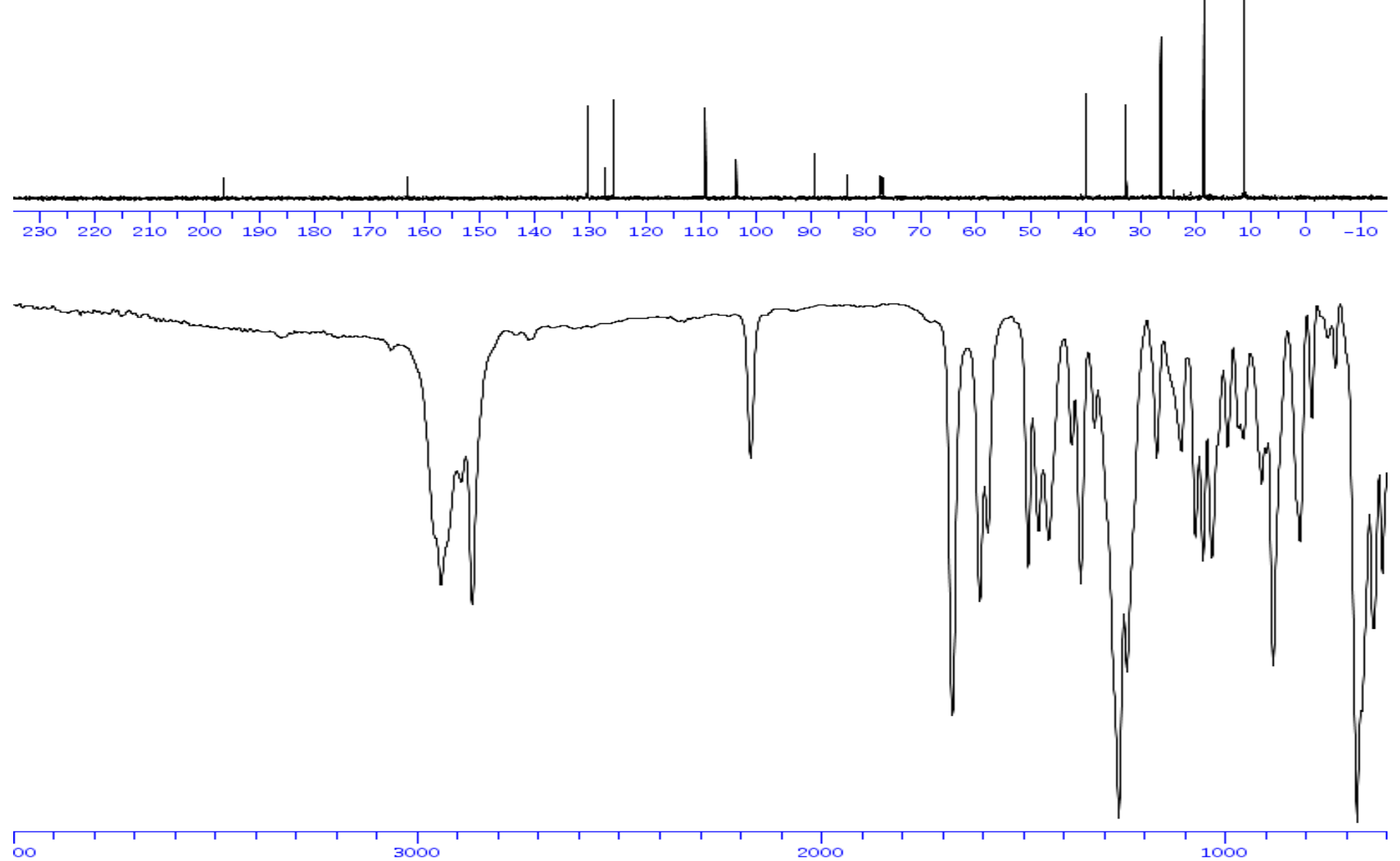
solvent: $<C D C I 3>$
Frequency. $400.13 \mathrm{MHz}$

$\mathrm{Cr}^{\mathrm{Be}} \mathrm{Si}^{\mathrm{Si} \mathrm{Pr}_{3}}$

$4 \mathbf{i}$
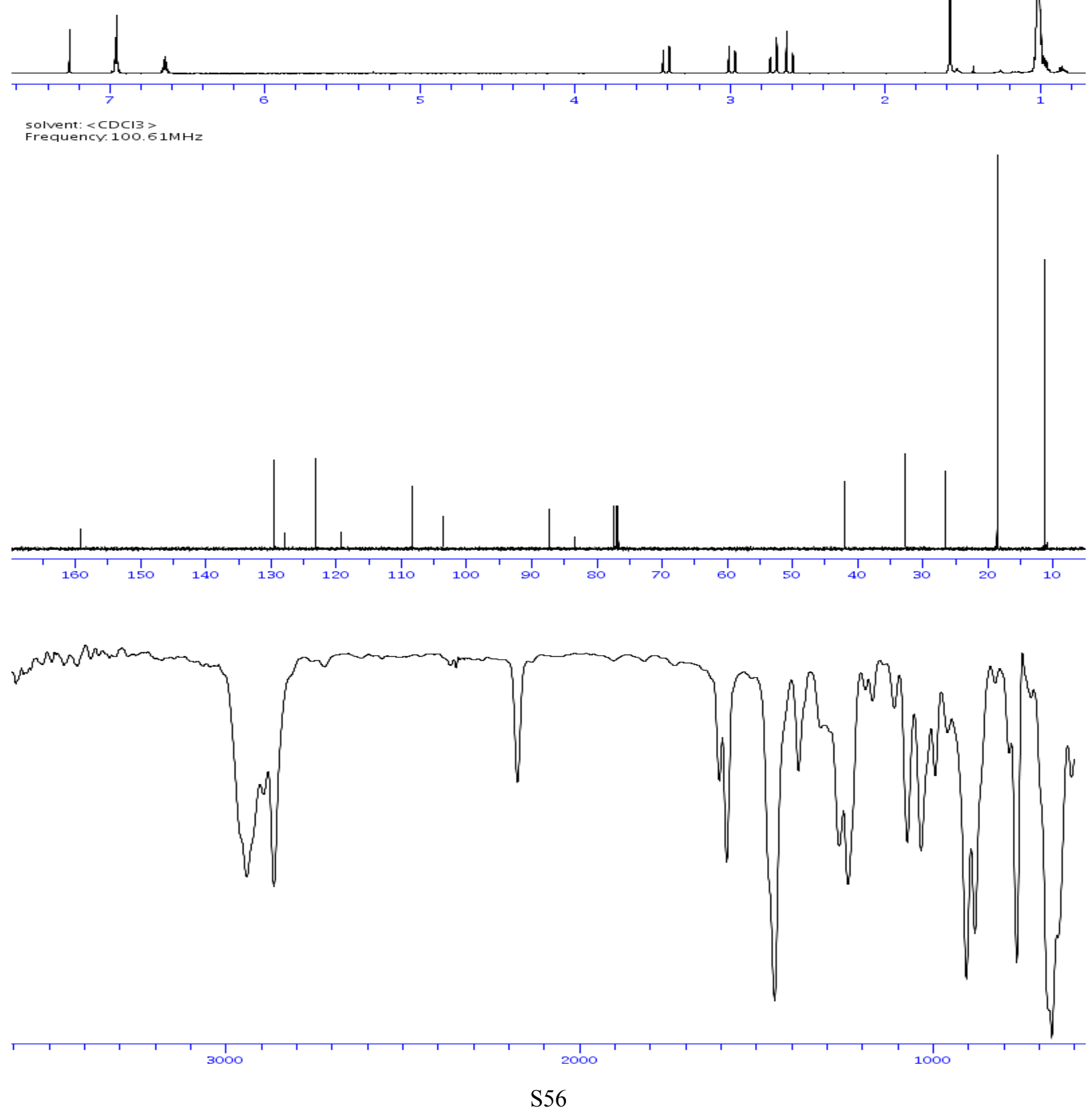


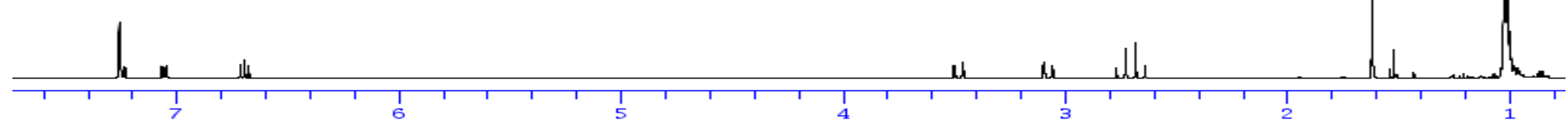

solvent: $\langle\mathrm{CDCI} 3\rangle$ Frequency. $100.61 \mathrm{MHz}$
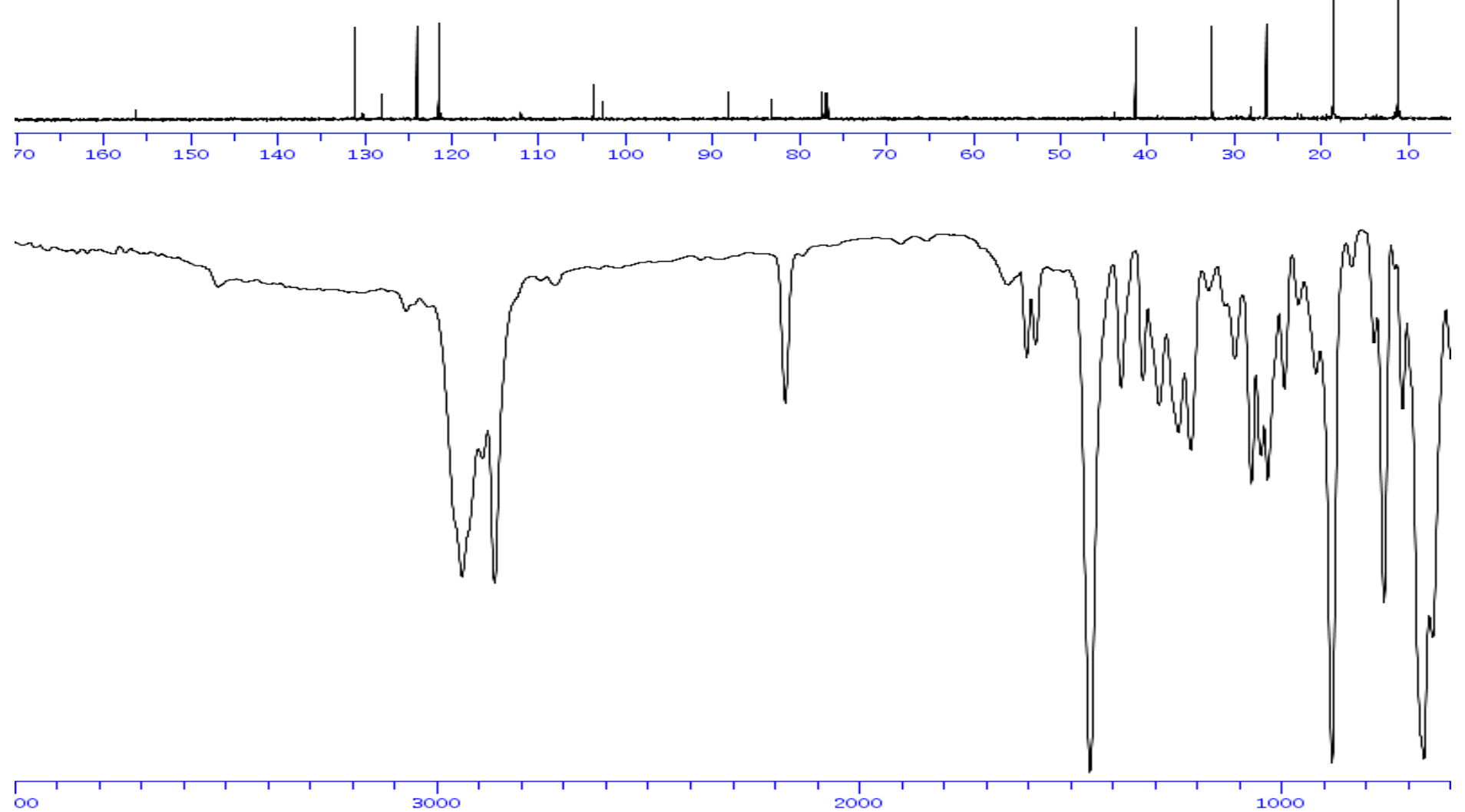

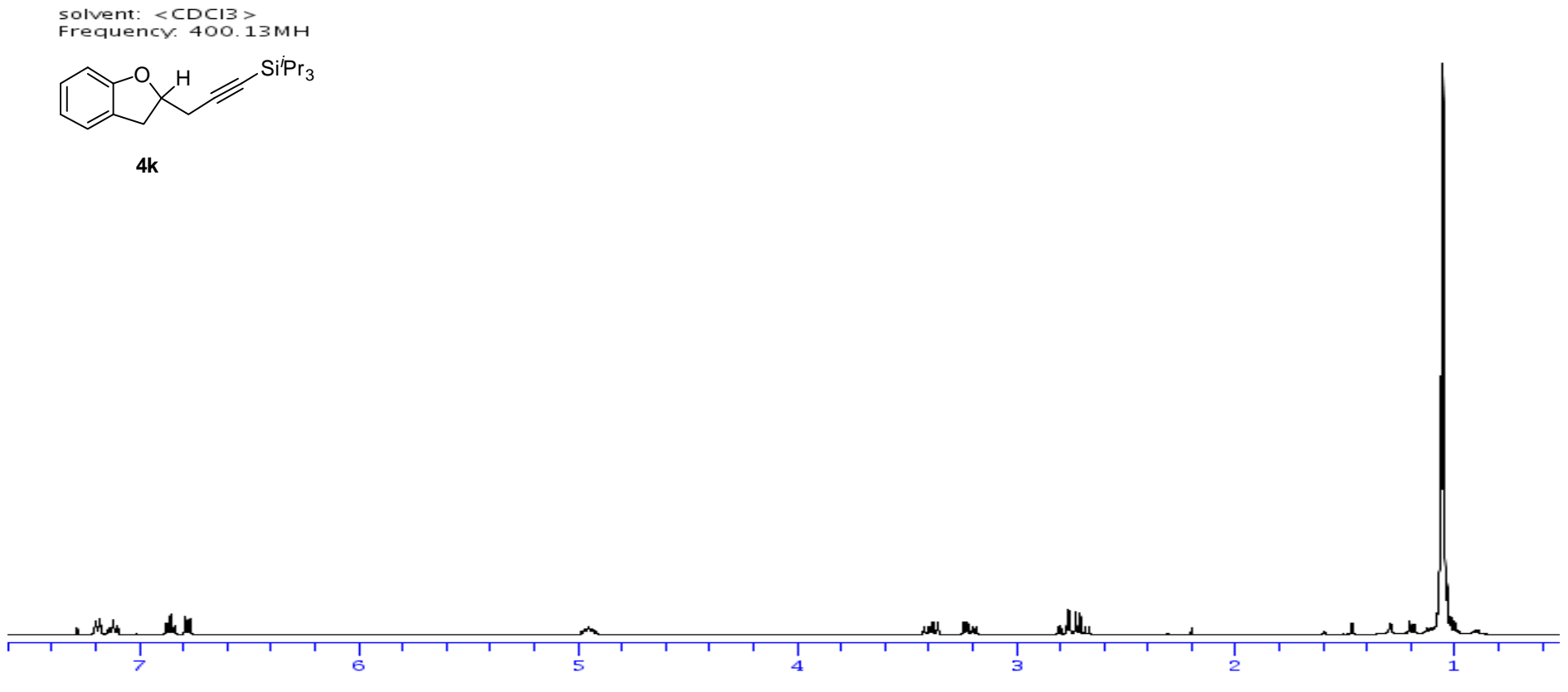

solvent: $<C D C I 3>$
Frequency. $100.61 \mathrm{MHz}$
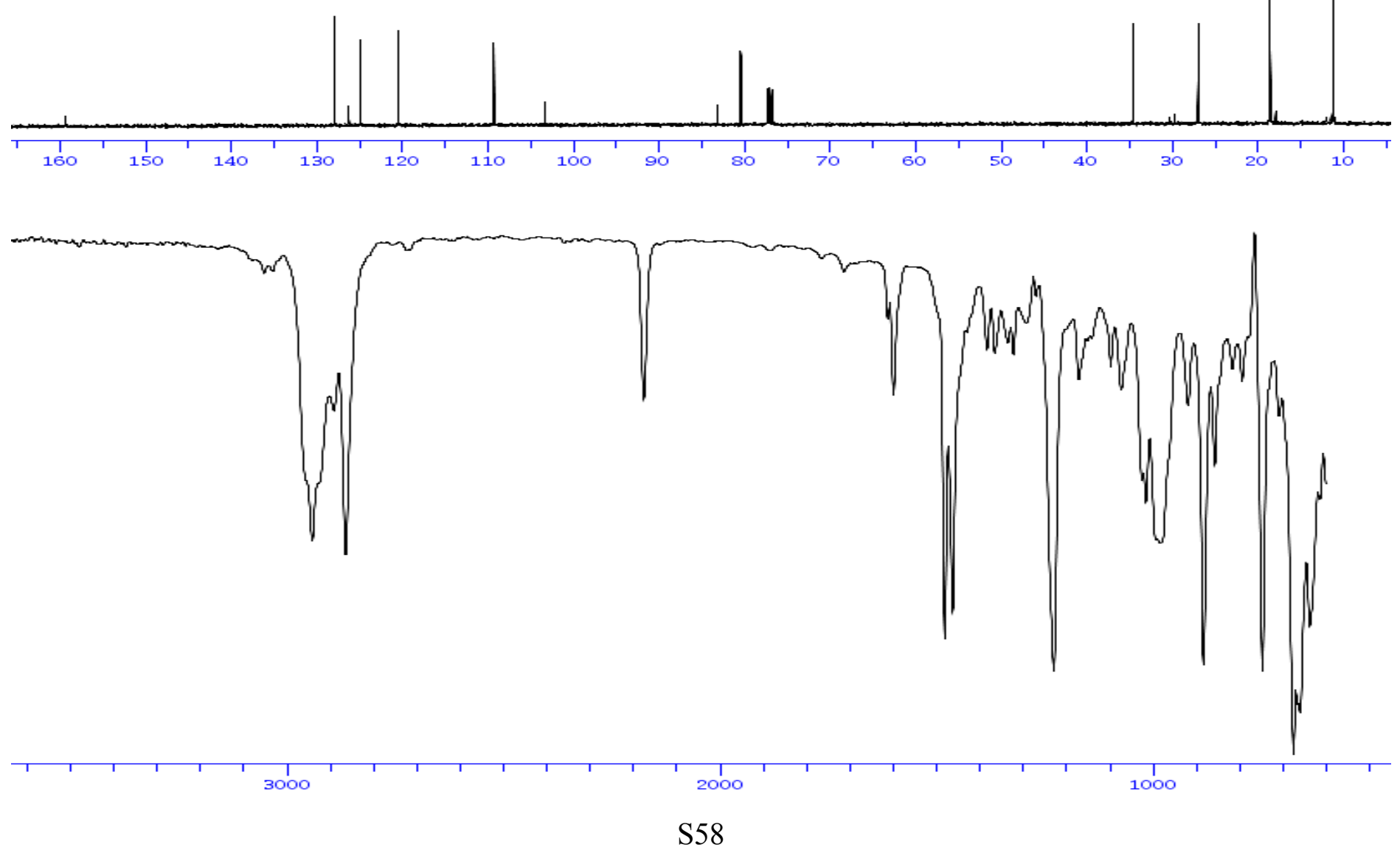


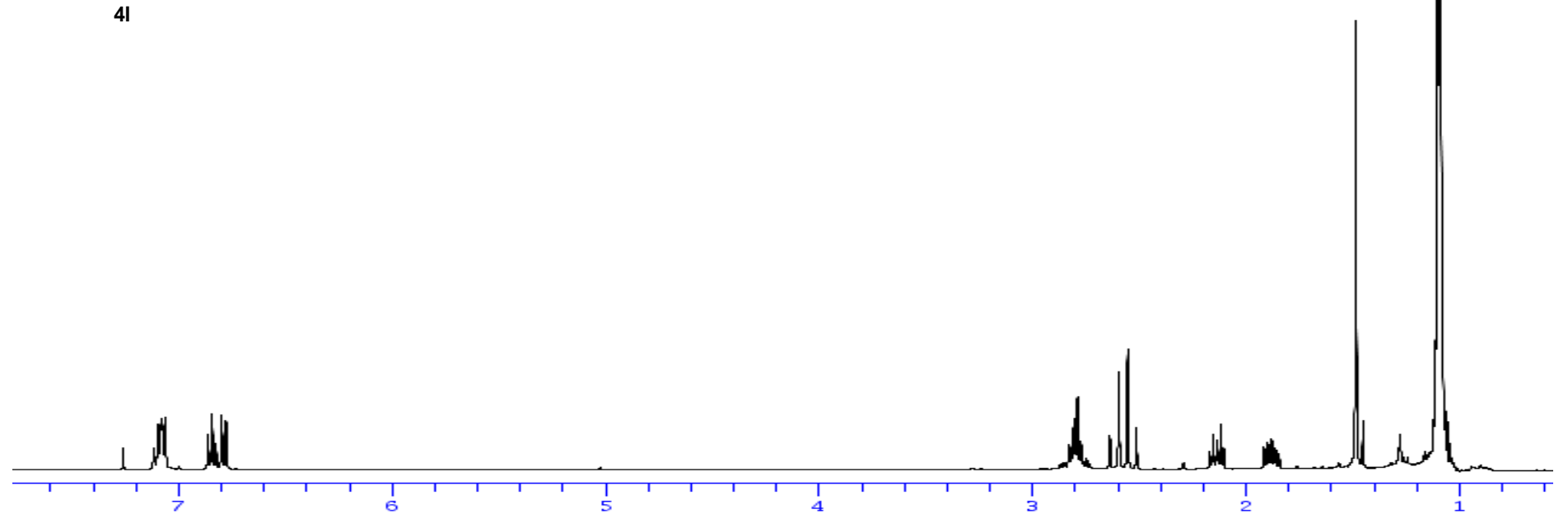

solvent: $\langle\mathrm{CDCI} 3\rangle$ Frequency. $100.61 \mathrm{MHz}$
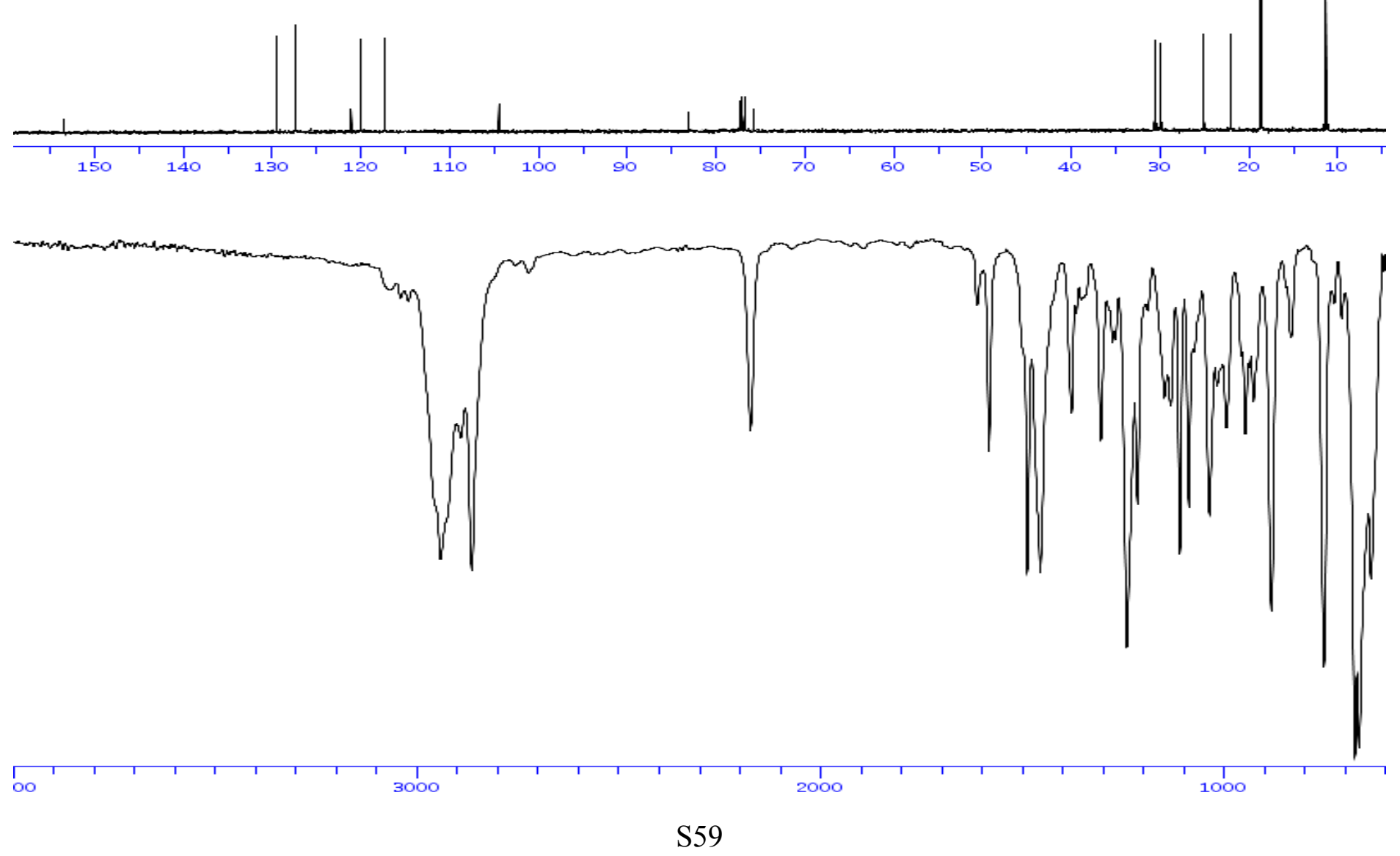
solvent: $<\mathrm{CDCI} 3>$

Frequency. $400.13 \mathrm{MHz}$

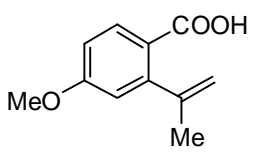

$5 b$

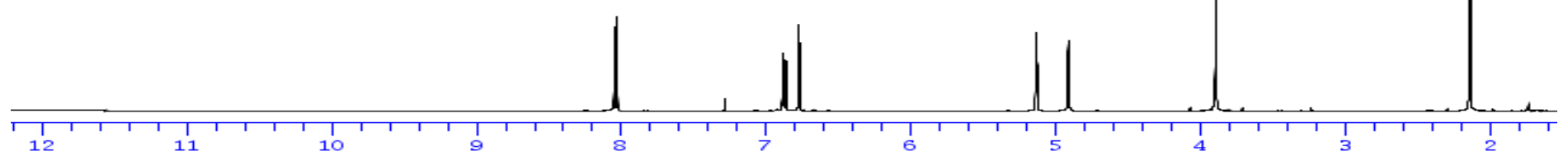

solvent: $<\mathrm{CDCI} 3\rangle$

Frequency. $100.61 \mathrm{MHz}$
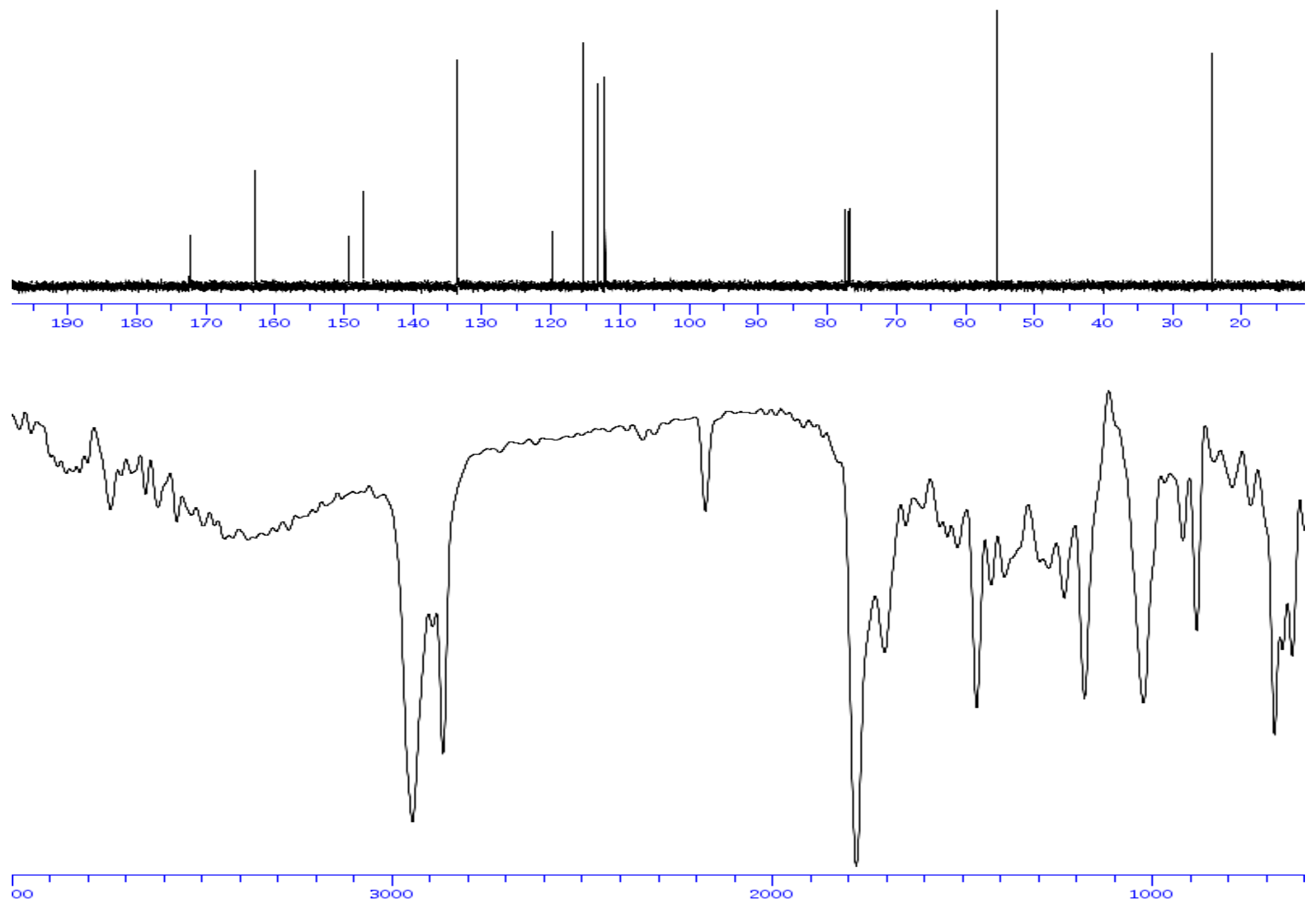
solvent: $<C D C I 3>$
Frequency. $400.13 \mathrm{MHz}$
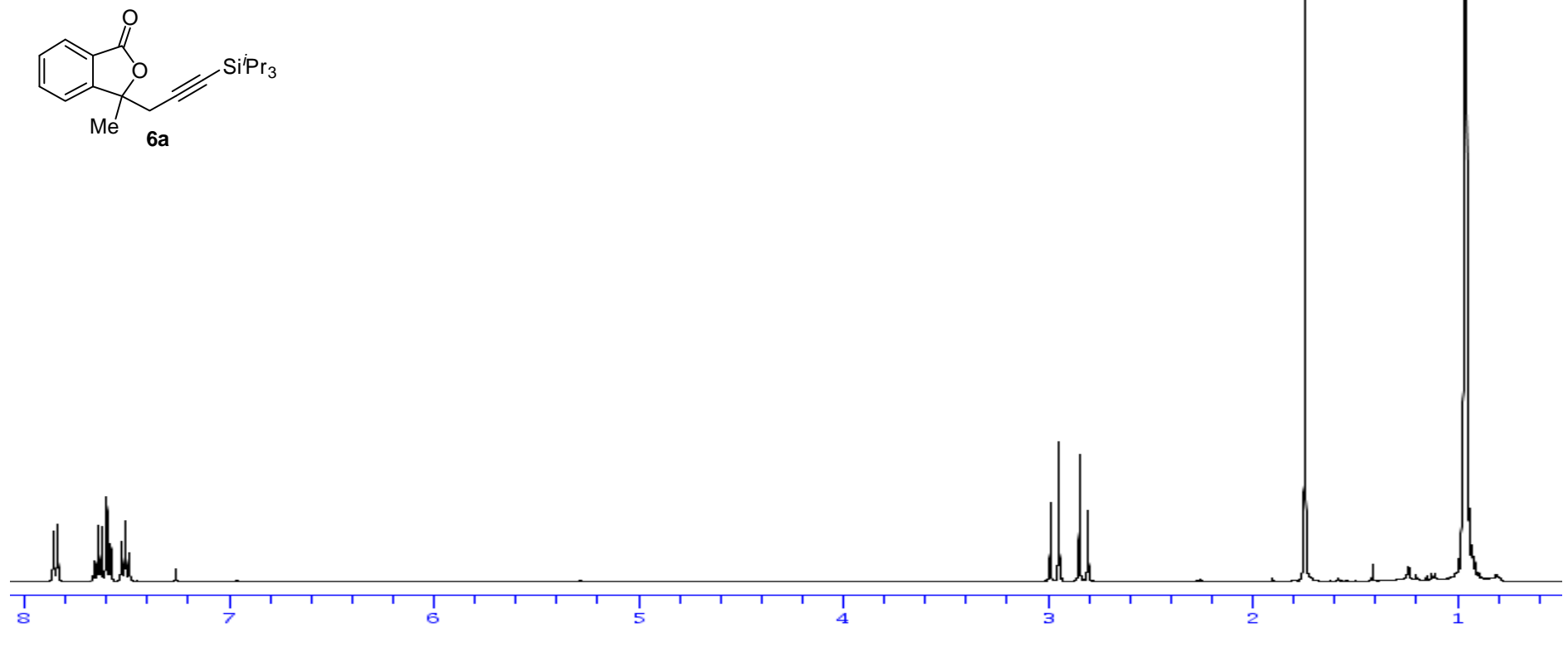

solvent: $<\mathrm{CDCI} 3>$
Frequency. $100.61 \mathrm{MHz}$
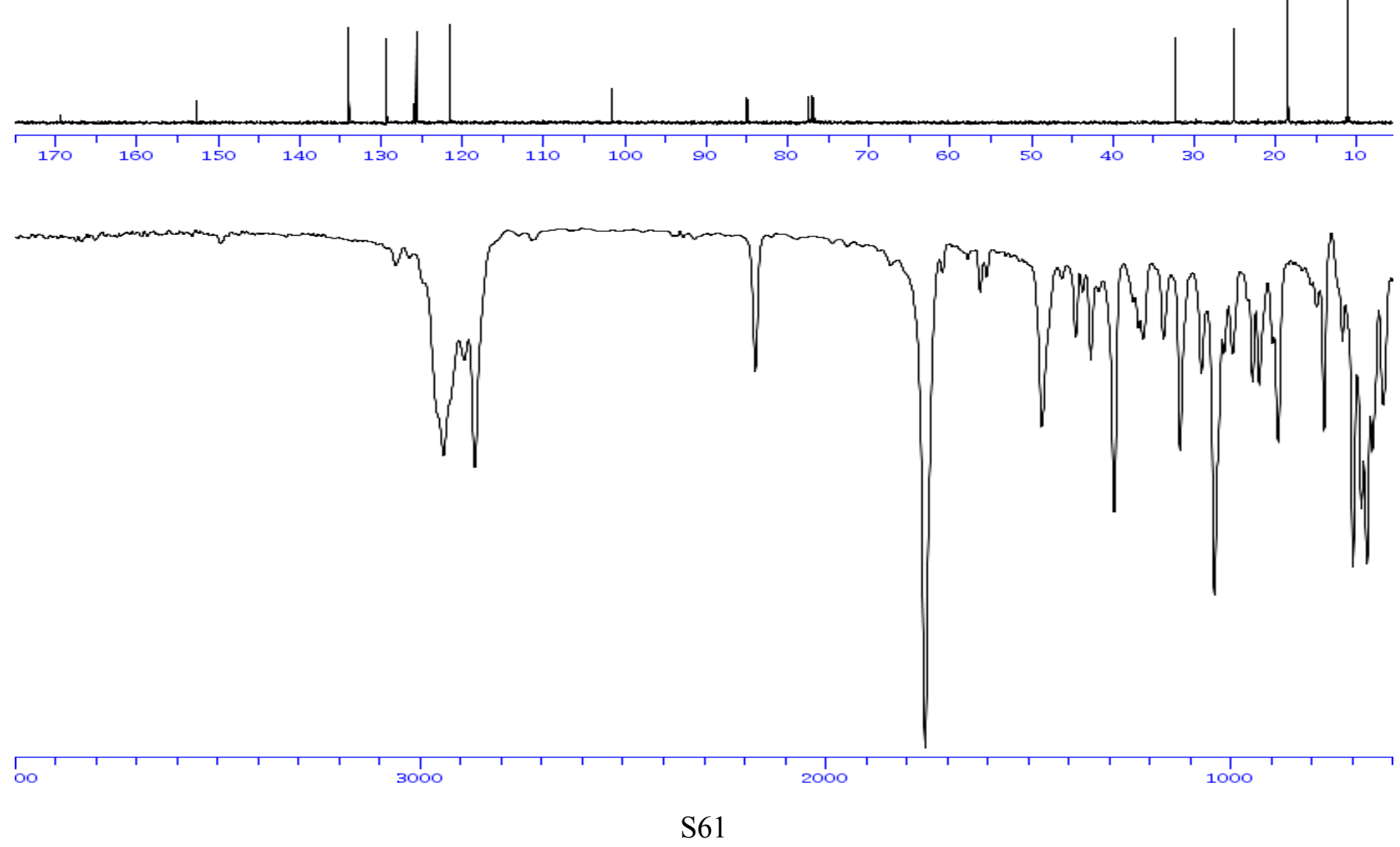
solvent: $<\mathrm{CDCI}>$

Frequency. $400.13 \mathrm{MHz}$

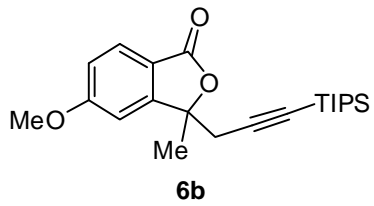

6b

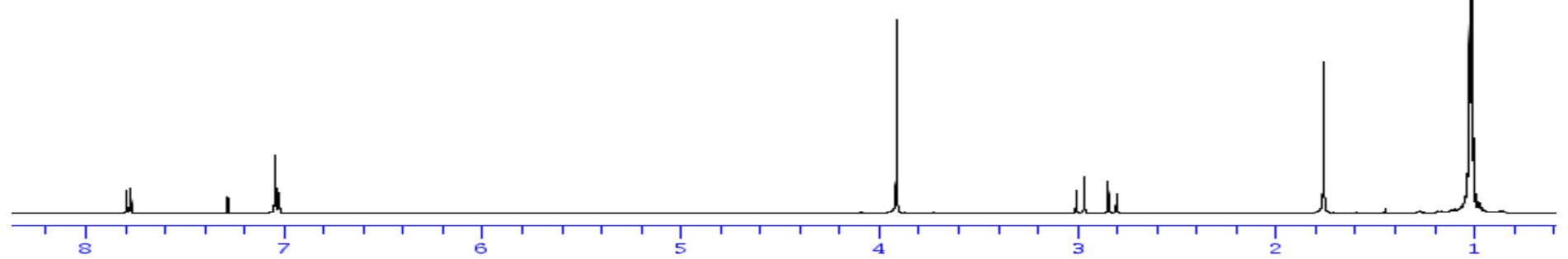

solvent: $<C D C I 3>$

Frequency. $100.61 \mathrm{MHz}$
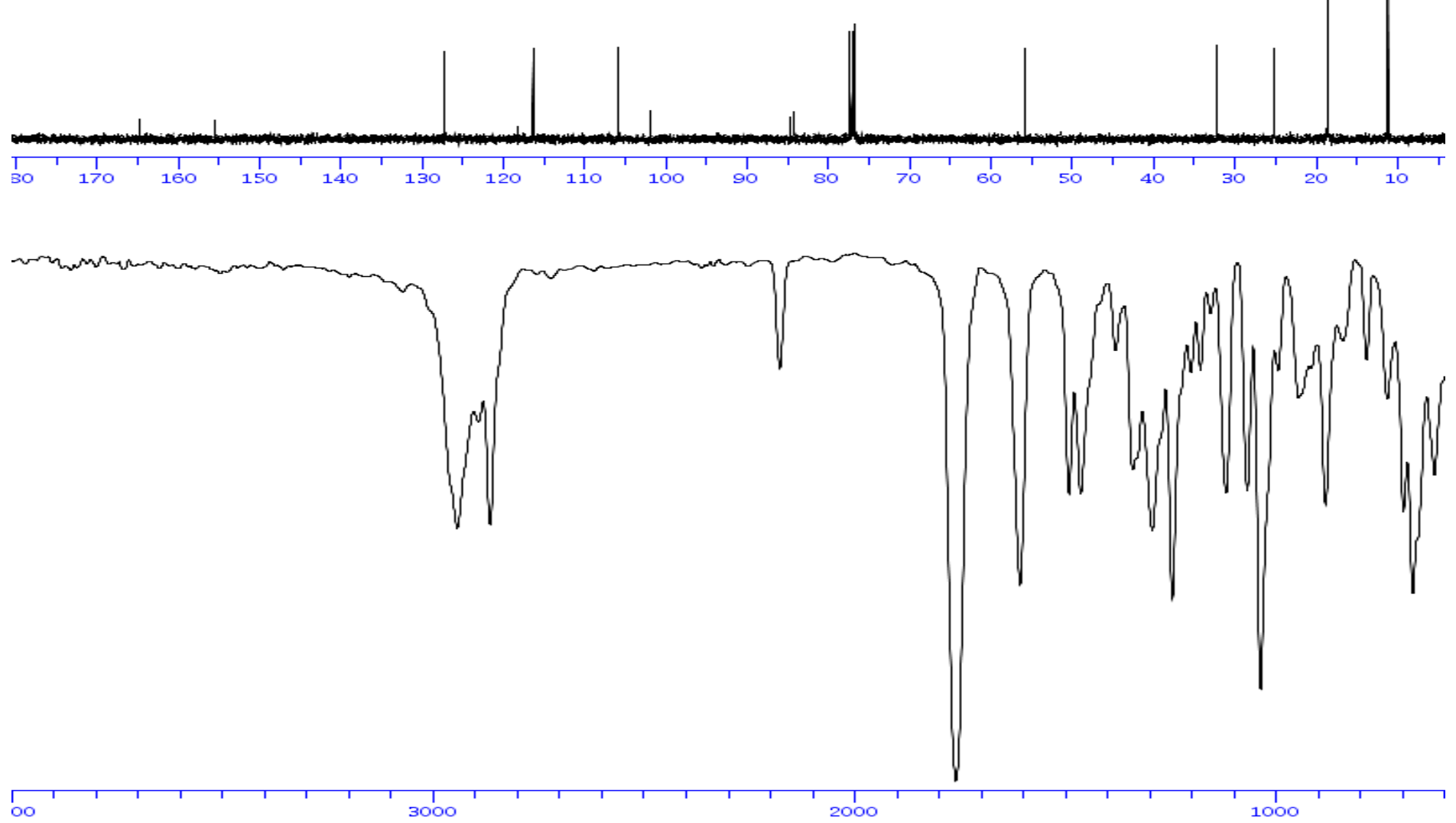

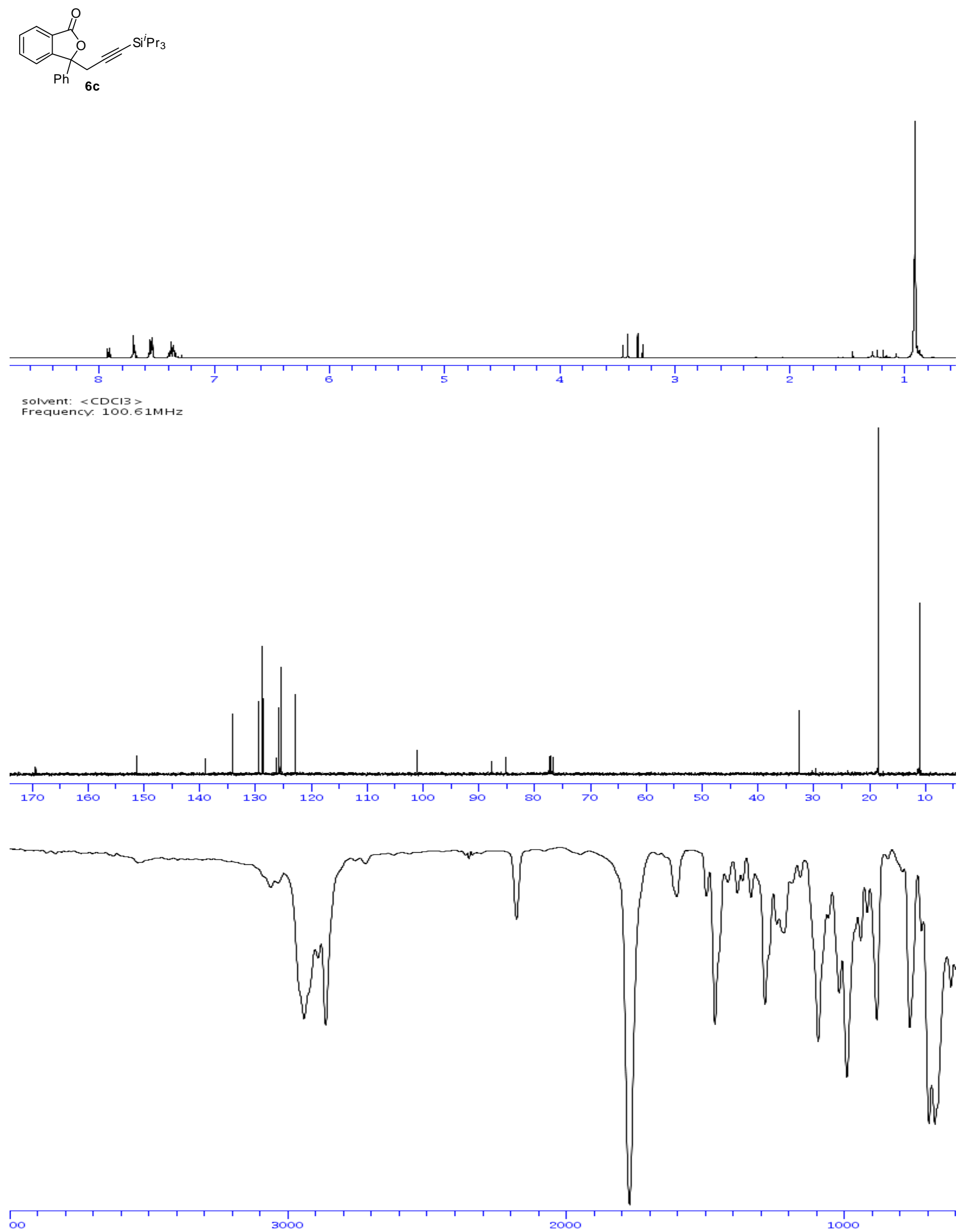

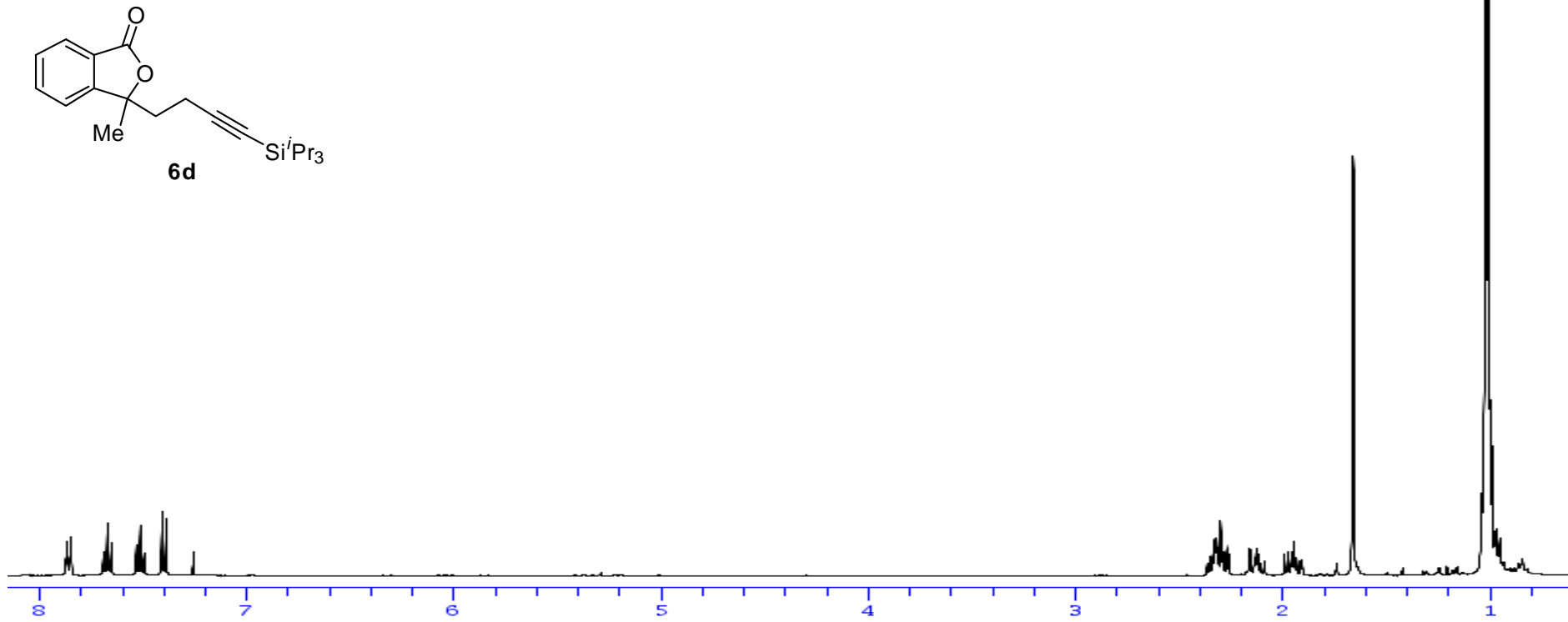

solvent: $<\mathrm{CDCI} 3\rangle$

Frequency. $10.61 \mathrm{MHz}$
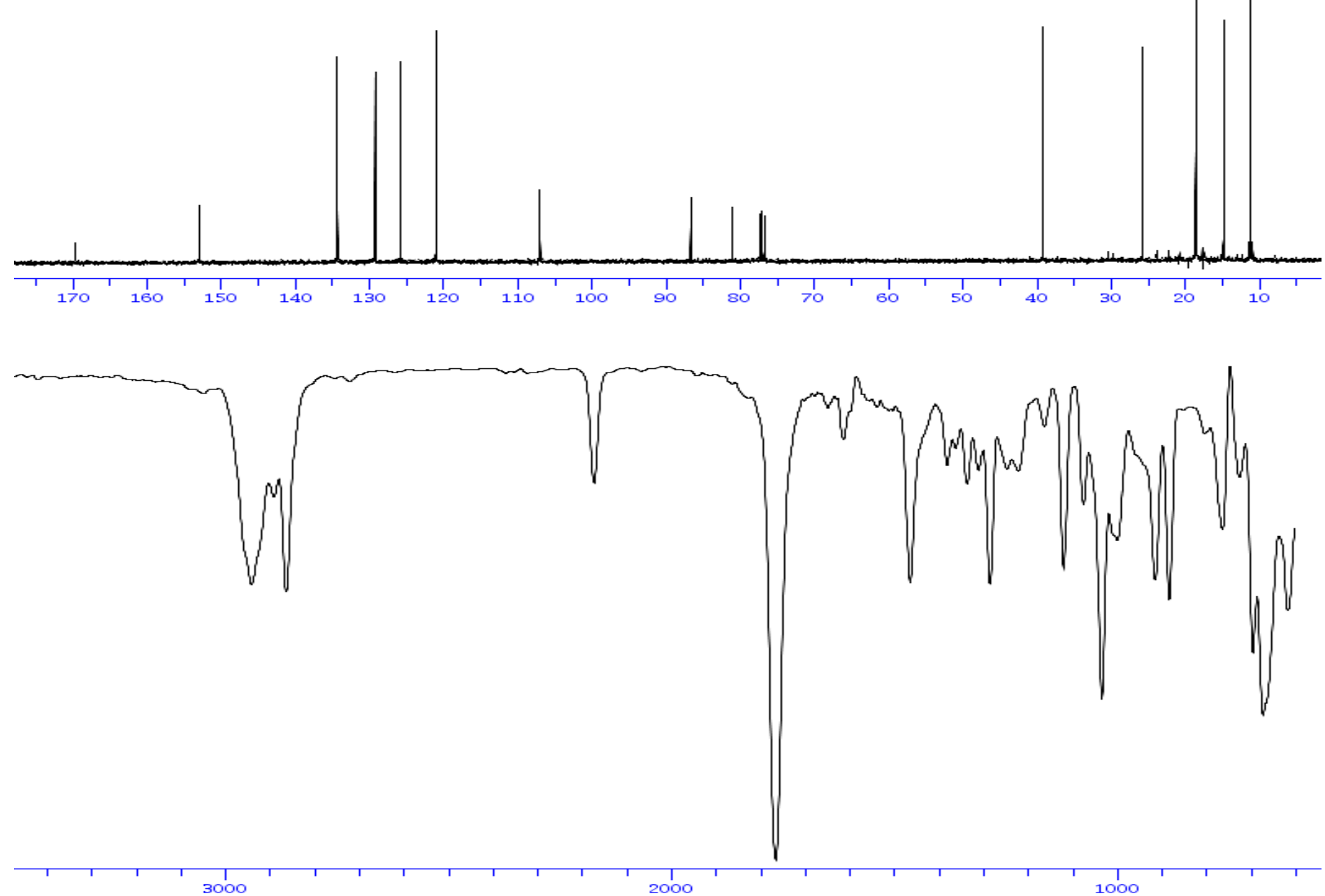

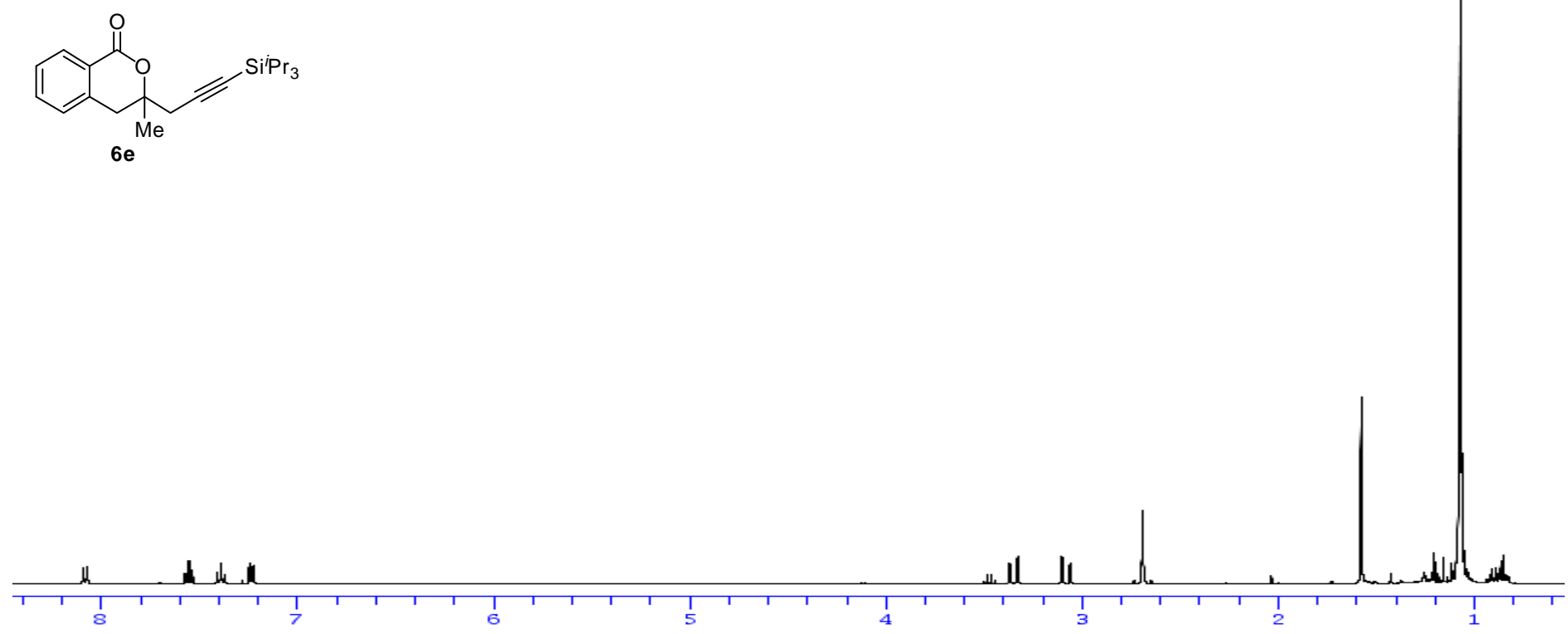
solvent : $\angle \mathrm{CDCI} 3>$ Frequency. 400.13 $\mathrm{MHz}$
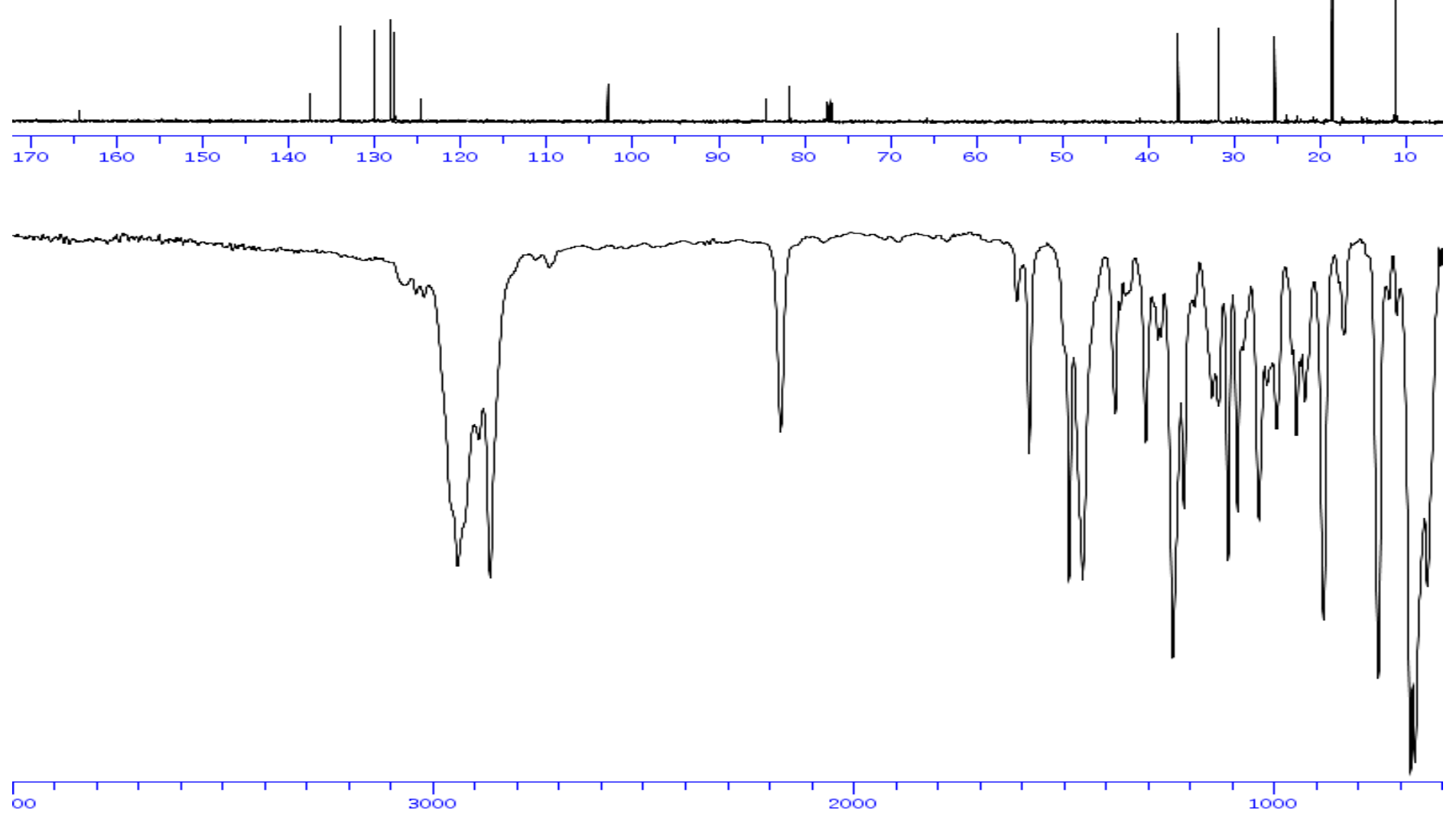
solvent: $<\mathrm{CDCI} 3>$

Frequency. 400.13 $\mathrm{MHz}$
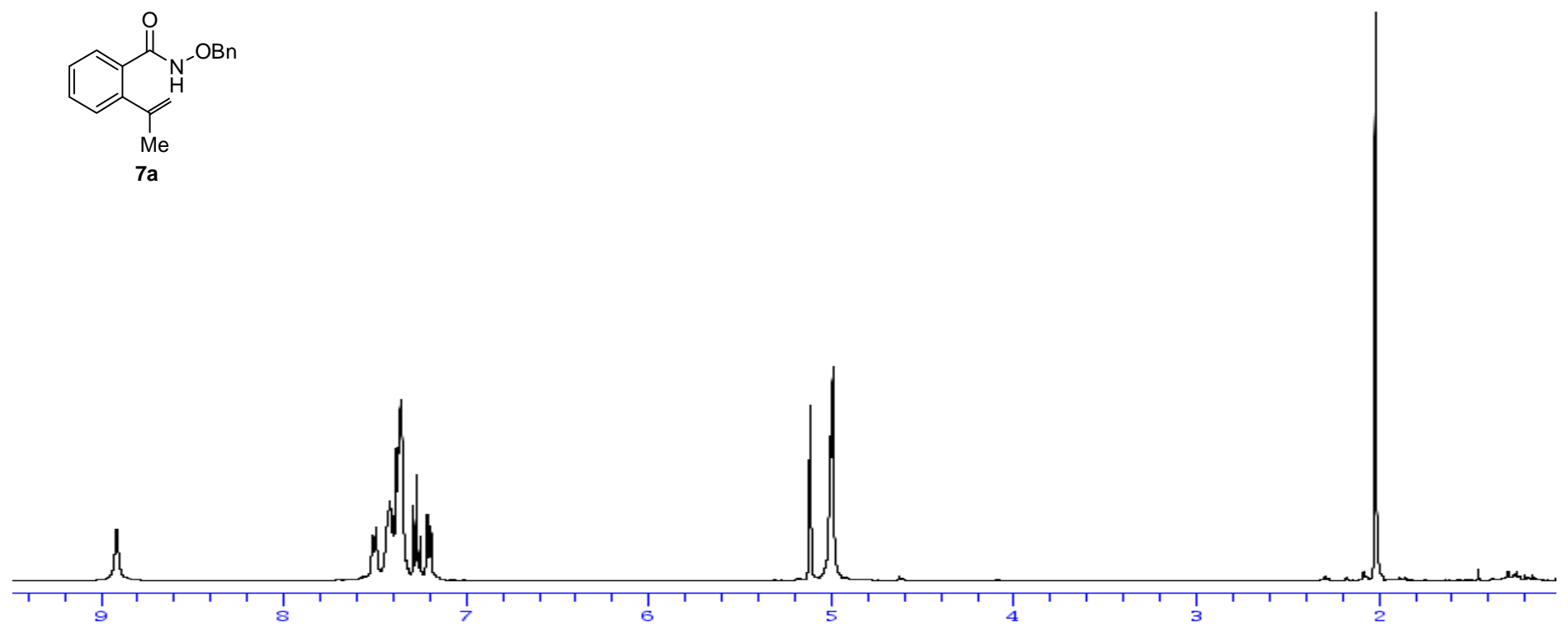

solvent: $<\mathrm{CDCI} 3>$

Frequency. $100.61 \mathrm{MHz}$
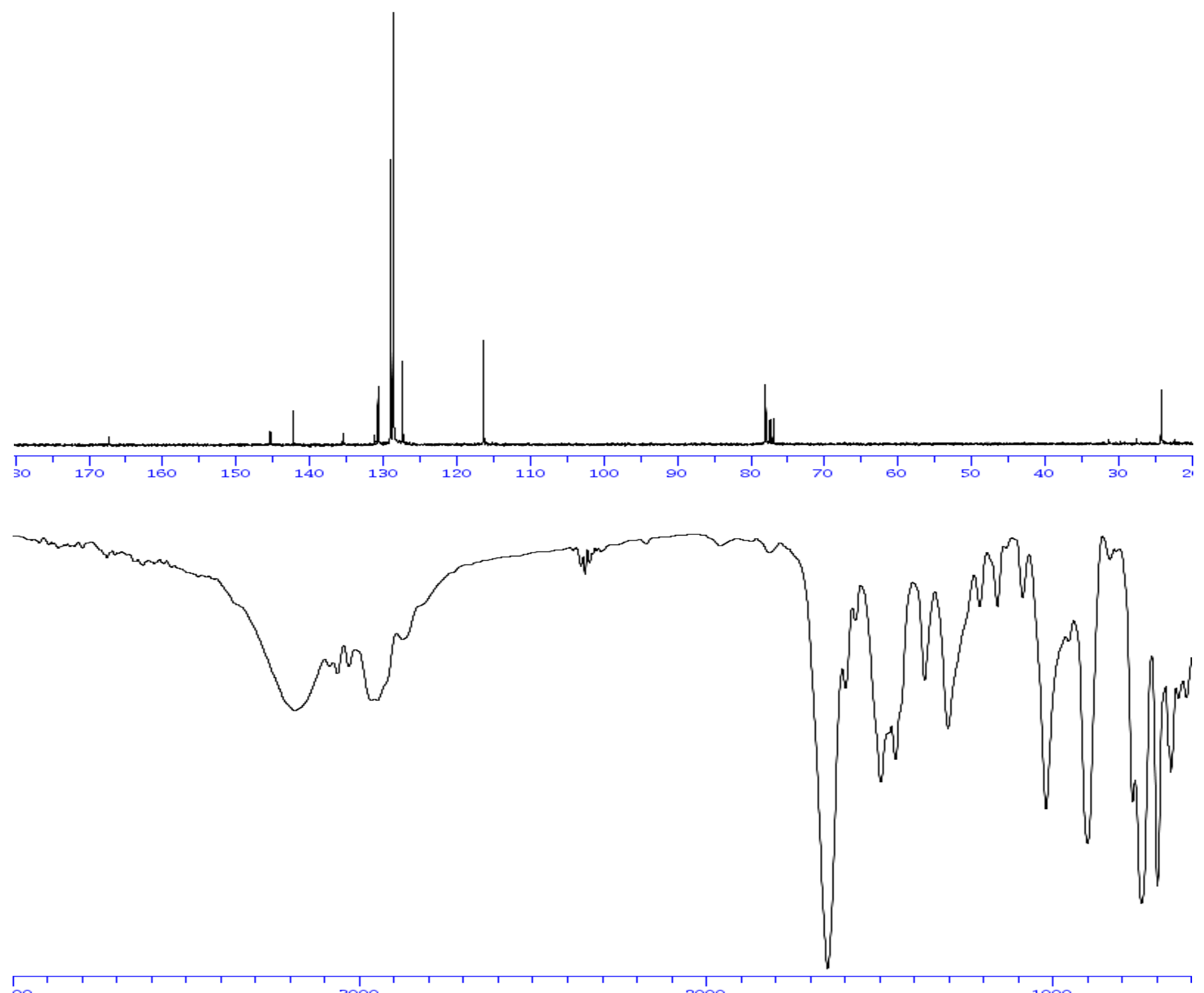


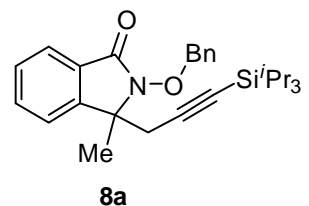

$8 a$

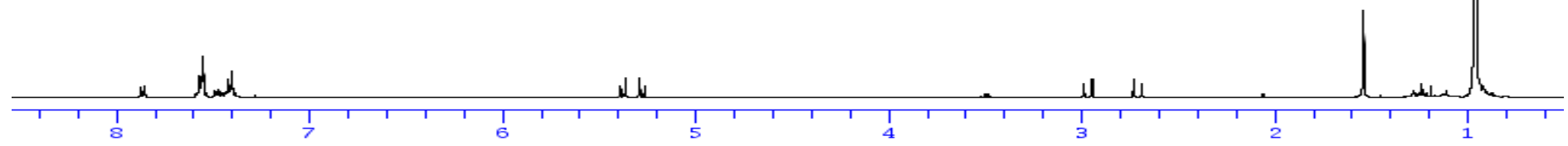

solvent: $\langle\mathrm{CDCI} 3\rangle$
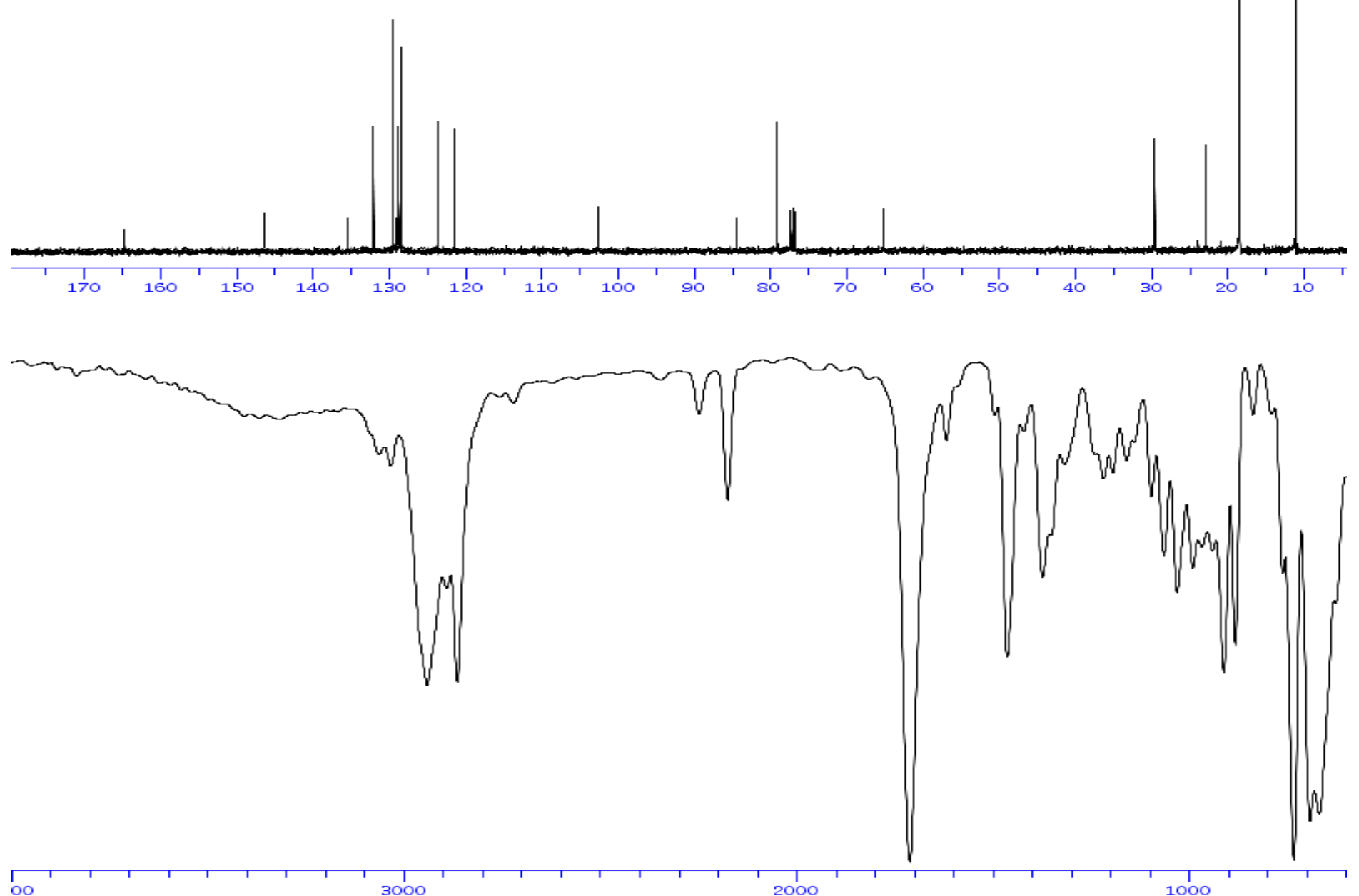
solvent: $<\mathrm{CDCI} 3>$

Frequency. 40O. $13 \mathrm{MHz}$

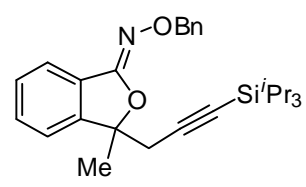

$8 b$

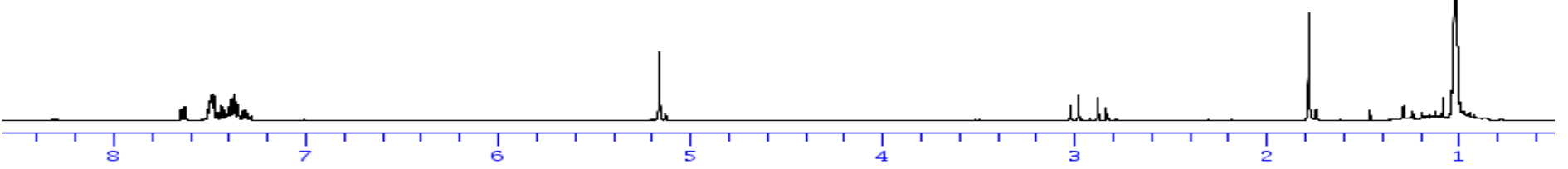

solvent: $\langle\mathrm{CDCI} 3\rangle$

Frequency. $100.61 \mathrm{MHz}$
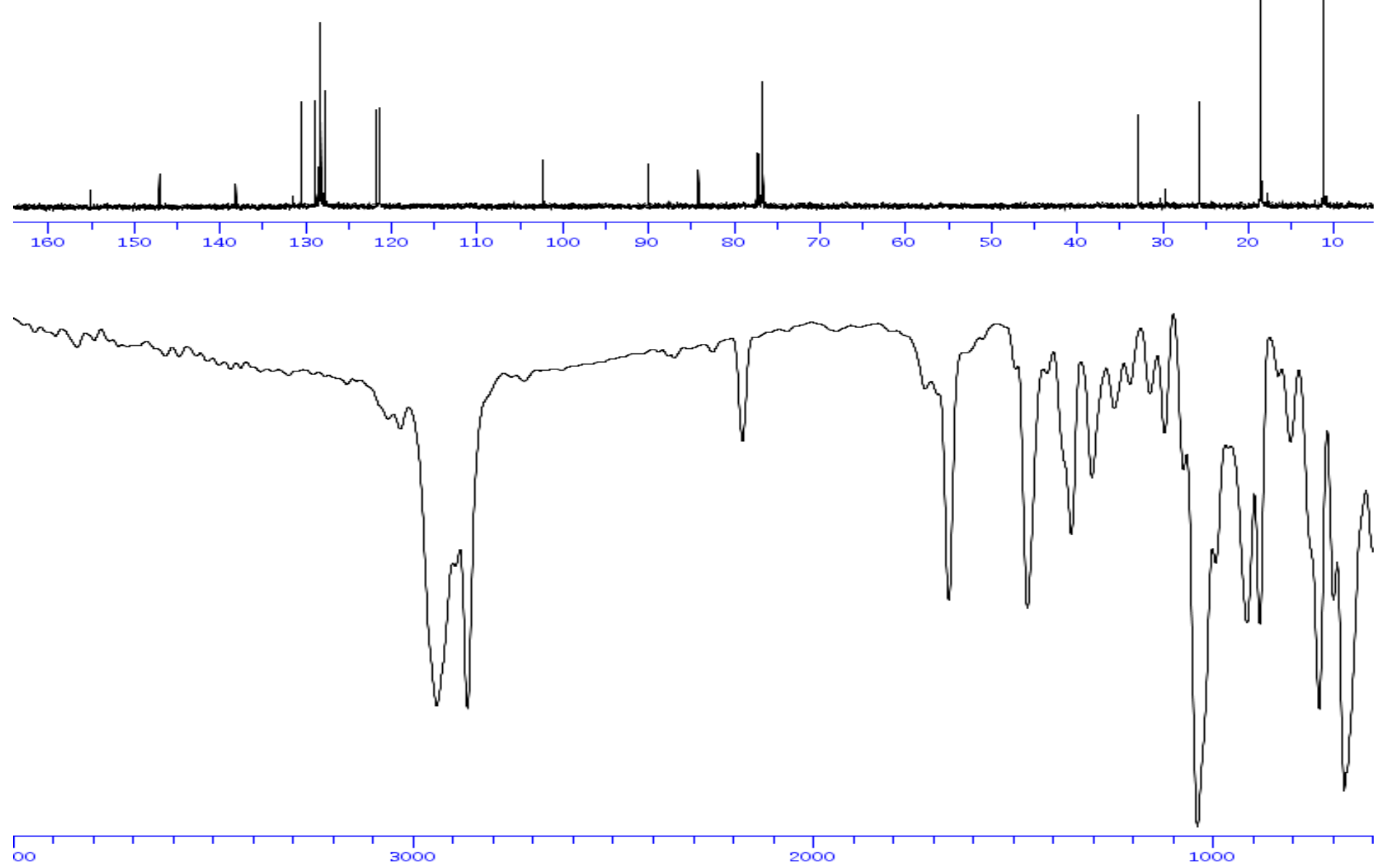
solvent: $<C D C L 3>$

Frequency. 40O. 13 $\mathrm{MHz}$
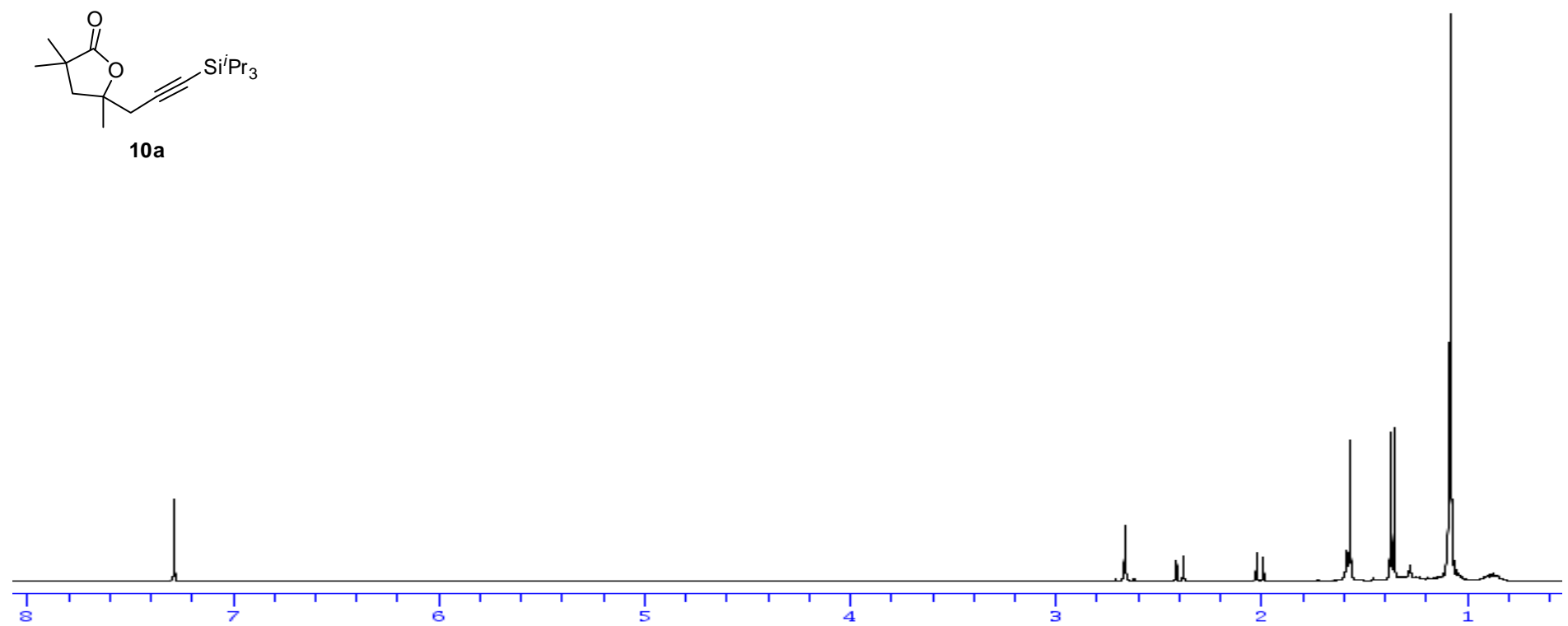
solvent: $<\mathrm{CDCI}$ > Frequency. $100.61 \mathrm{MHz}$
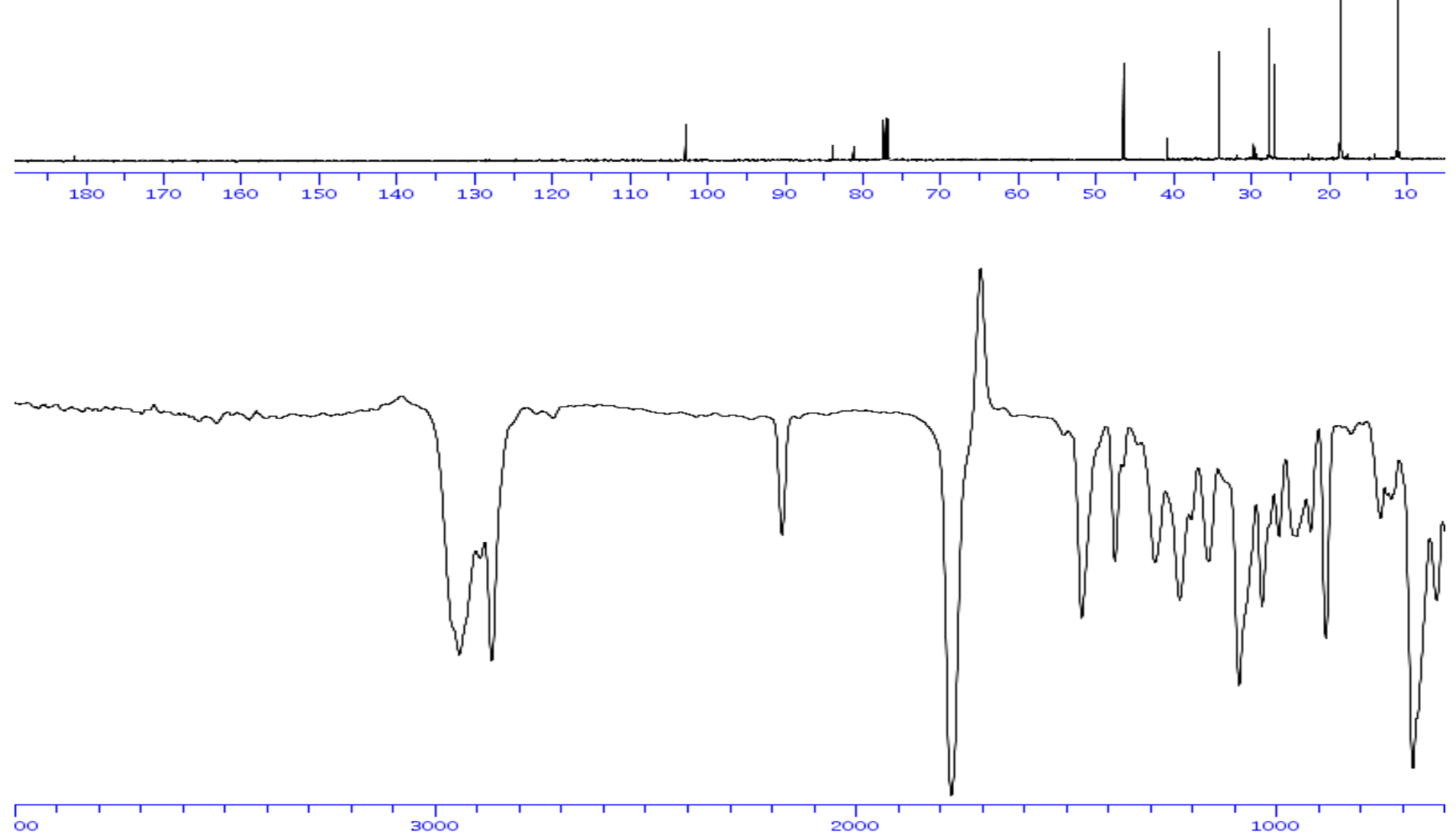
solvent: $<\mathrm{CDCI} 3>$

Frequency. 400.13 $\mathrm{MHz}$

$\underbrace{C_{1}^{O i P r} P_{3}}_{10 b}$

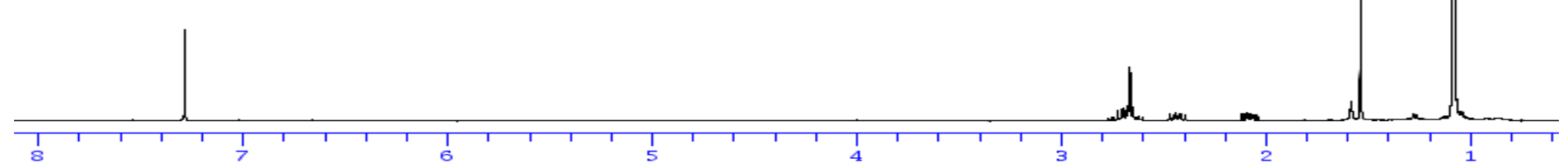

solvent: $\langle\mathrm{CDCI} 3\rangle$

Frequency. 100.75 $\mathrm{MHz}$
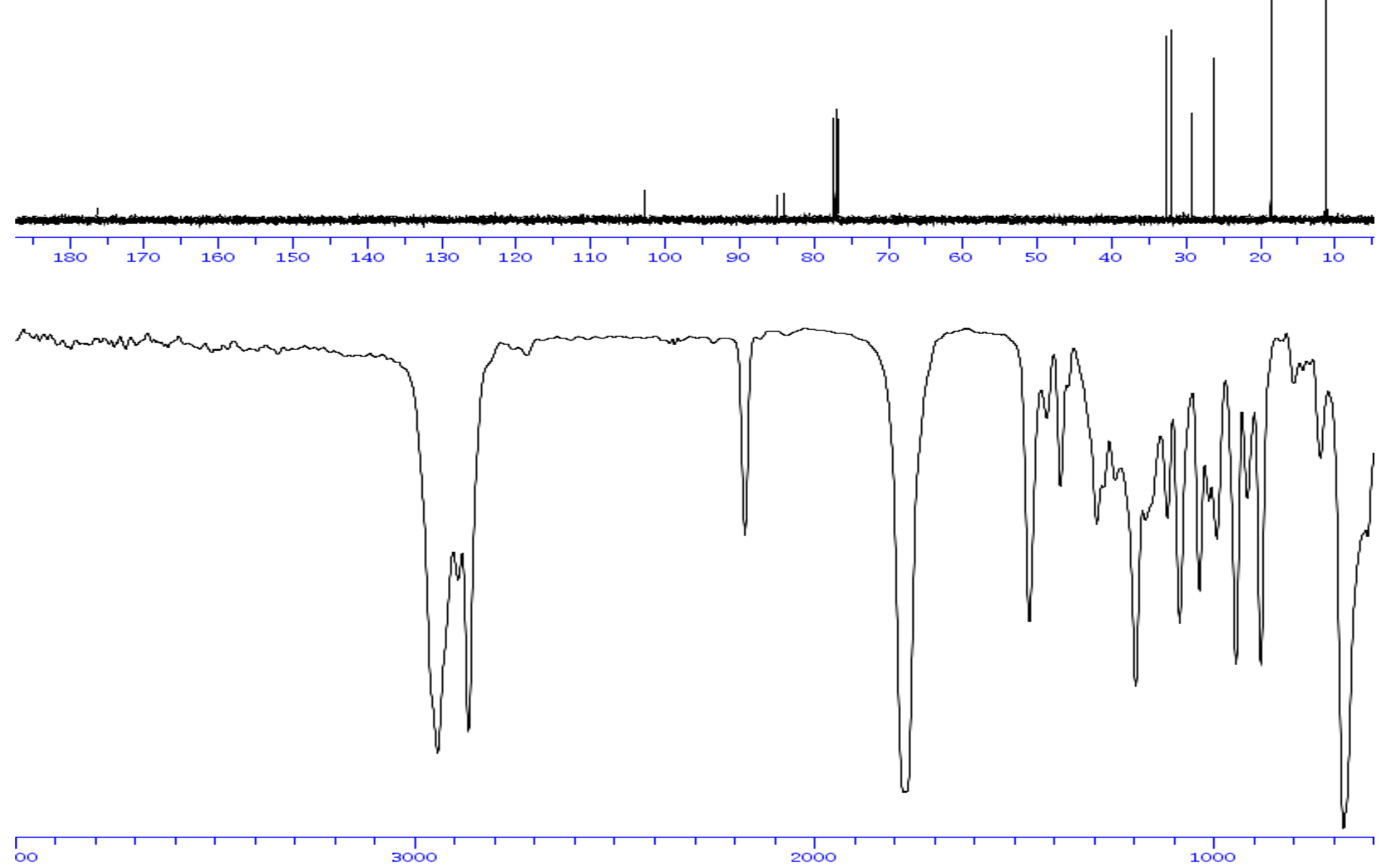
solvent: $<\mathrm{CDCI} 3>$

Frequency. $400.13 \mathrm{MHz}$

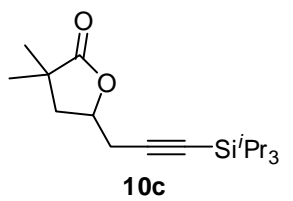

$10 c$

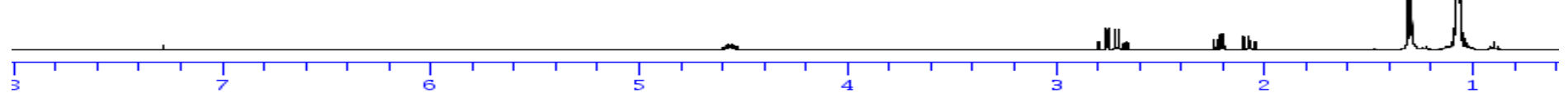

solvent: $\langle\mathrm{CDCI} 3\rangle$

Frequency. $100.75 \mathrm{MHz}$
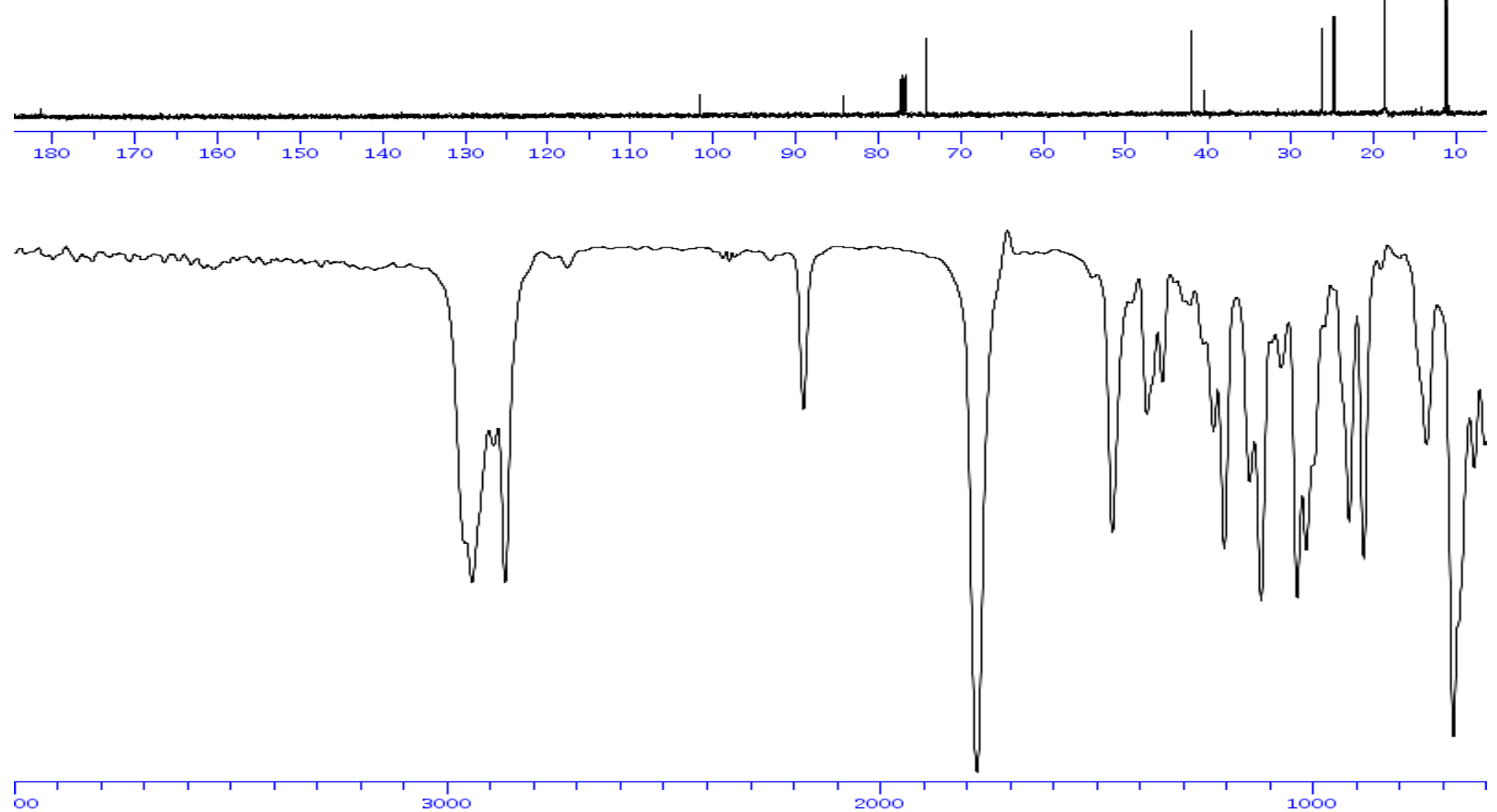
solvent: $<C D C I 3>$

Frequency.400.13 $\mathrm{MHz}$

$\underbrace{\overline{10 d}}_{\overline{10 d}} \operatorname{SiPr}_{3}$

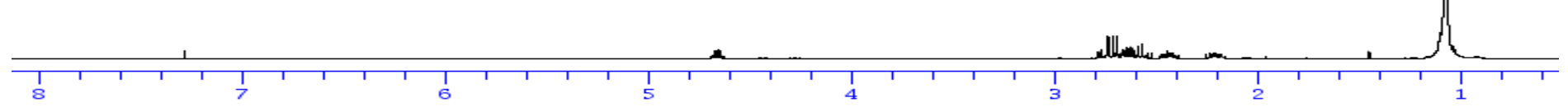

solvent: $<C D C I 3>$

Frequency. $100.61 \mathrm{MHz}$
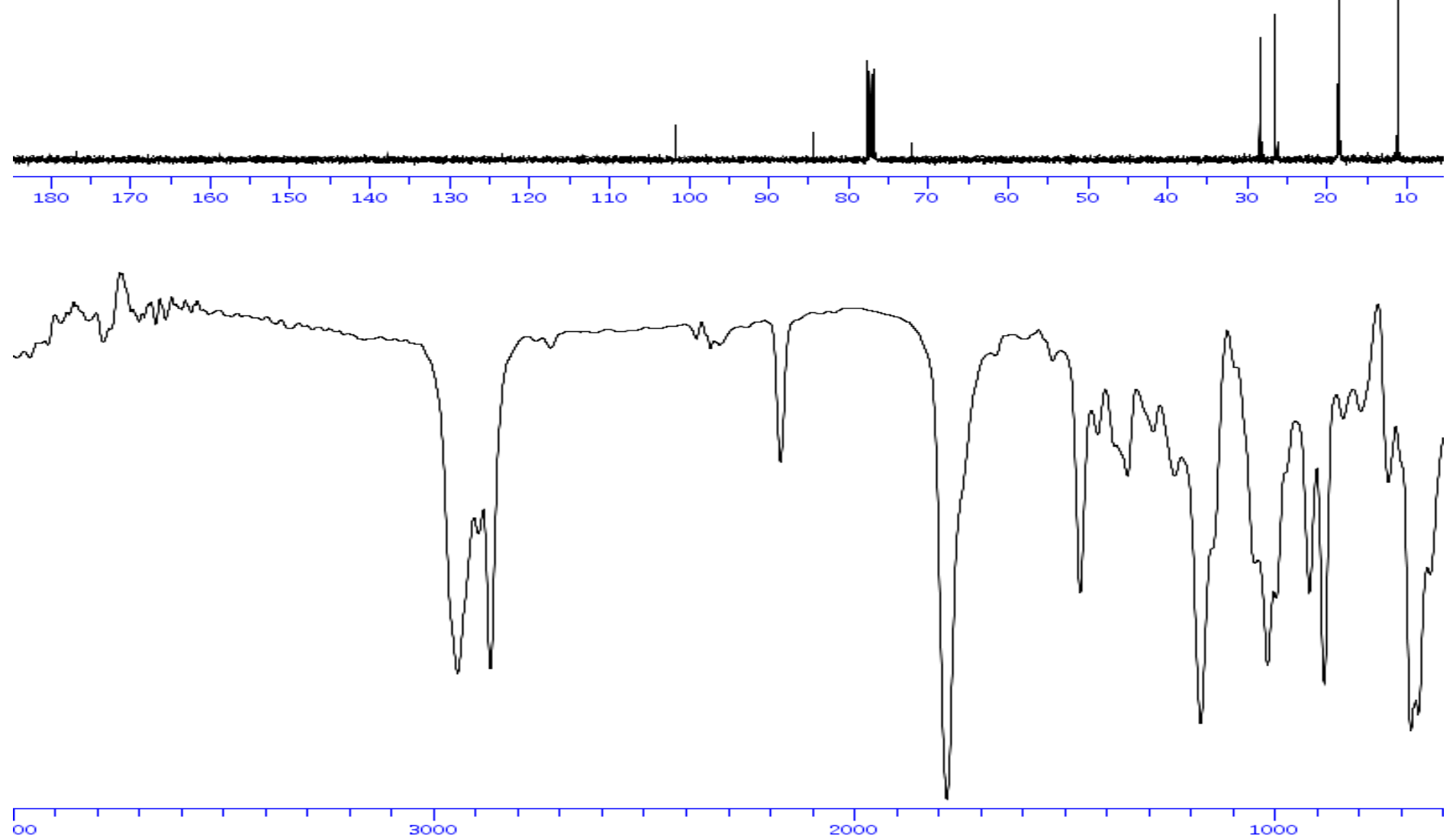

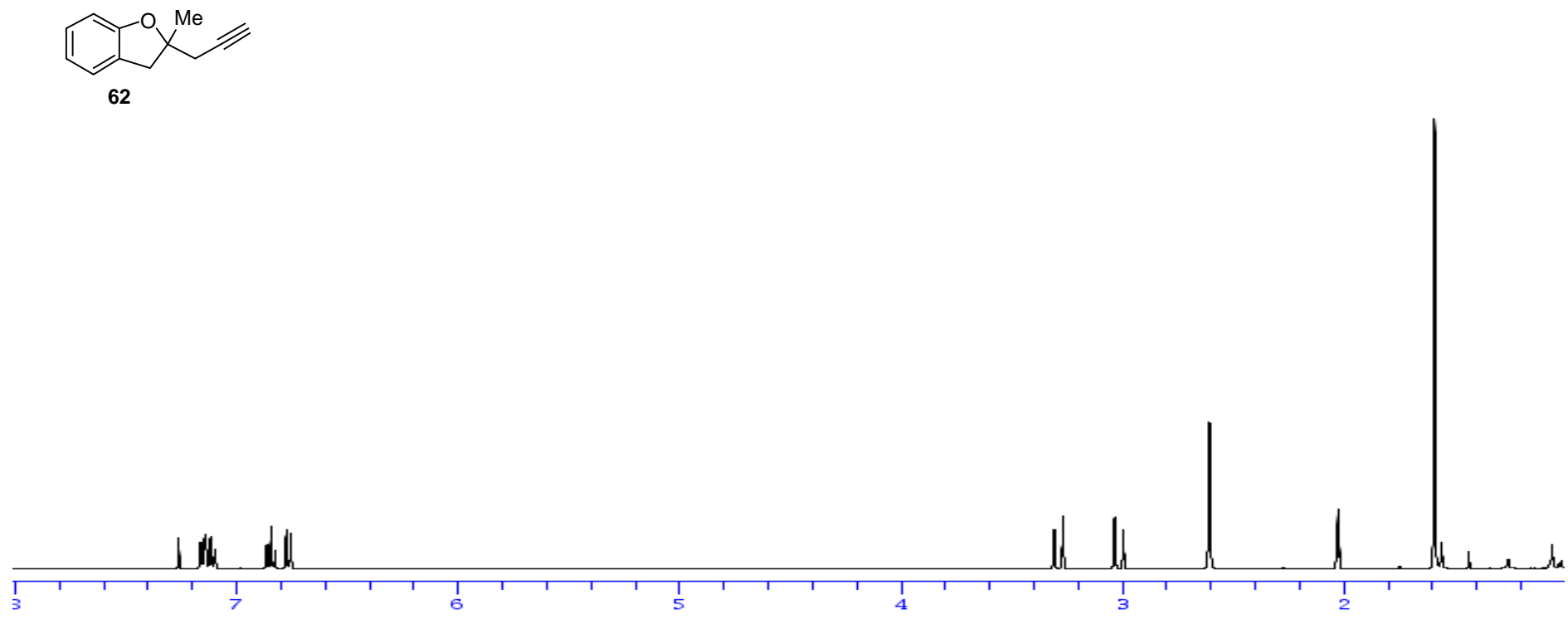
solvent: $\angle \mathrm{CDCI} 3\rangle$ Frequency. $100.61 \mathrm{MHz}$
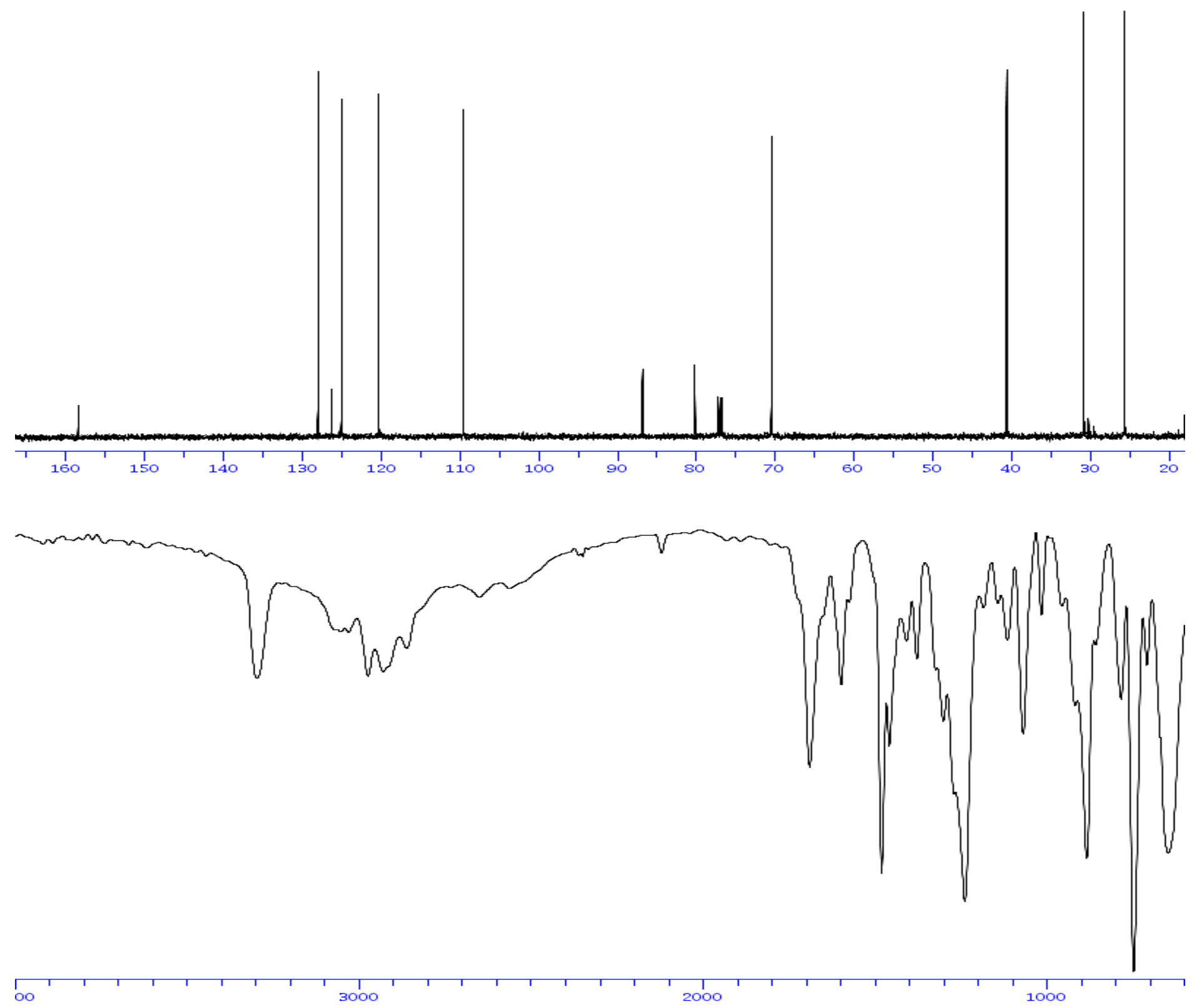
PMBO

$>13$

$60 \mathrm{Br}$

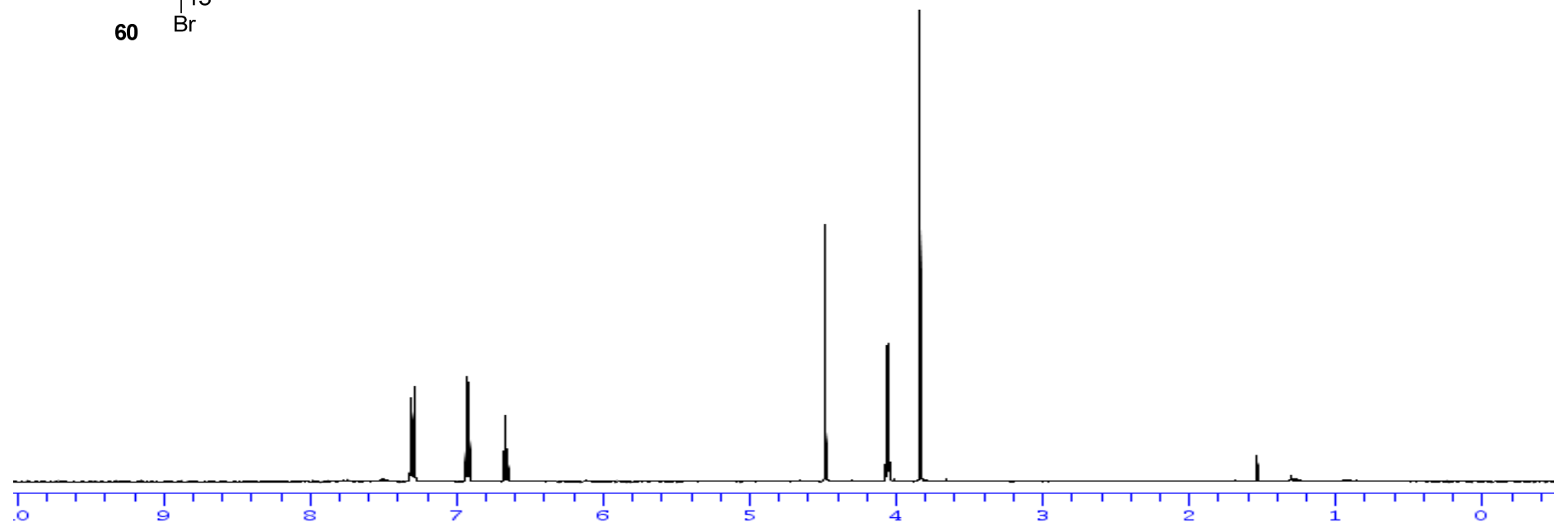

$\underbrace{\mathrm{PMBO}}_{60} \mathrm{Br}^{\mathrm{Br}}$

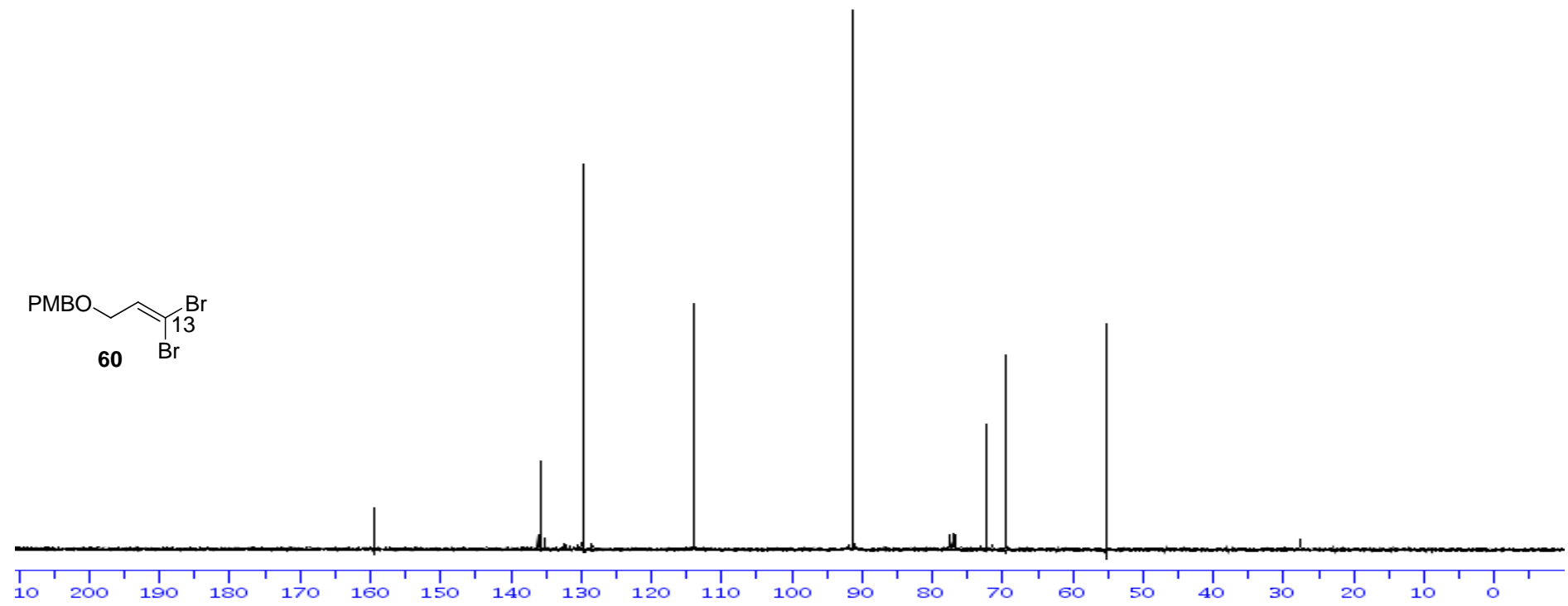

solvent: $<C D C I 3$

Frequency. $100.612769 \mathrm{MHz}$
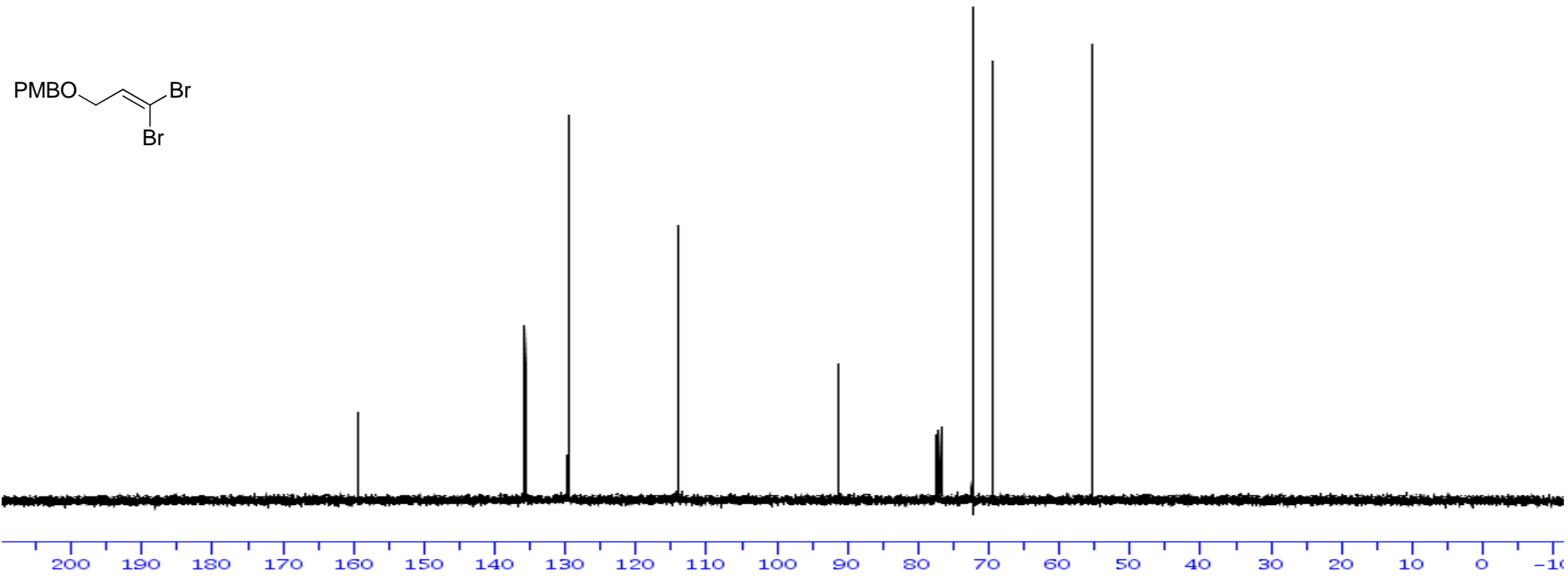


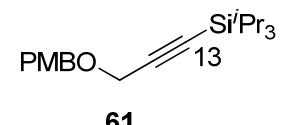

61
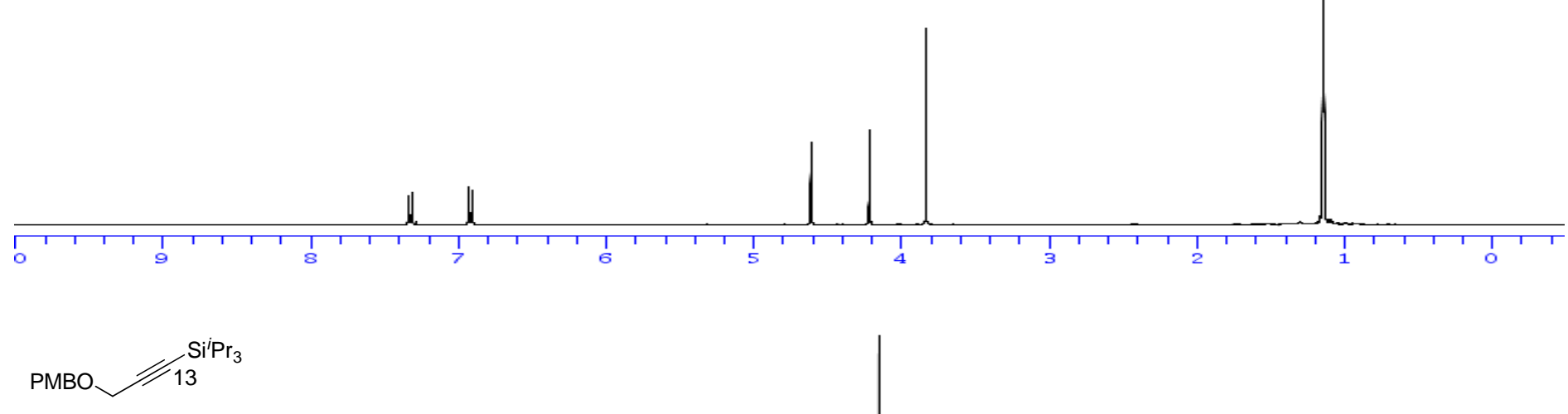

61
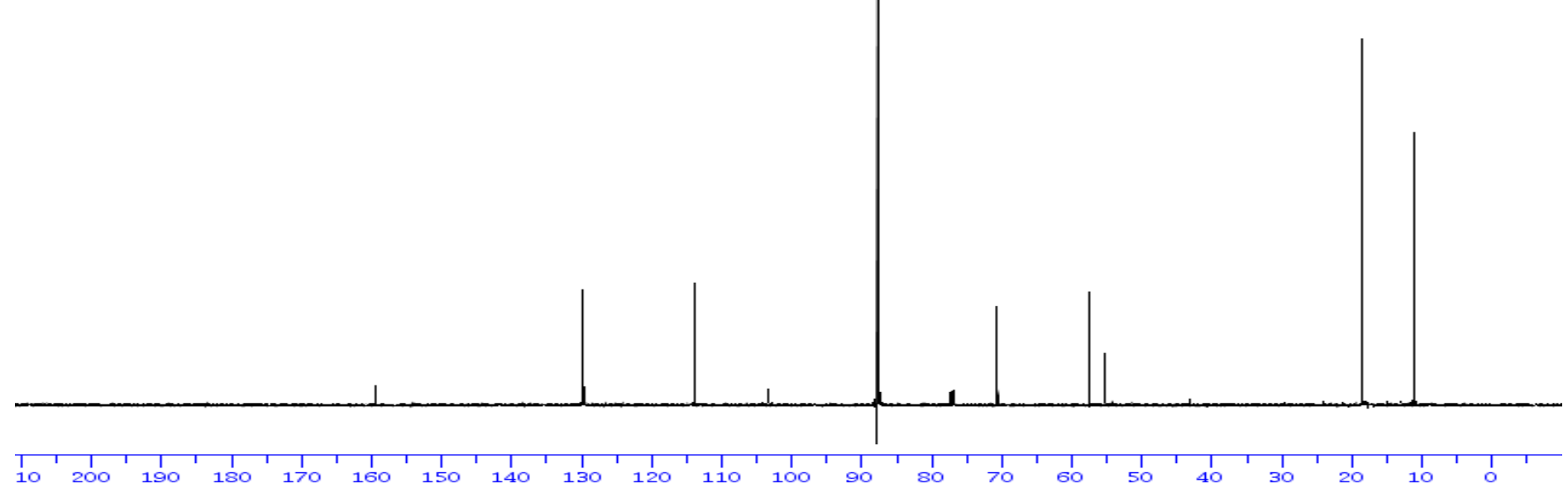

solvent: $\angle C D C I 3$

Frequency. $100.612769 \mathrm{MHz}$

$\mathrm{PMBO} / \mathrm{Si}^{\mathrm{Si}_{3}}$

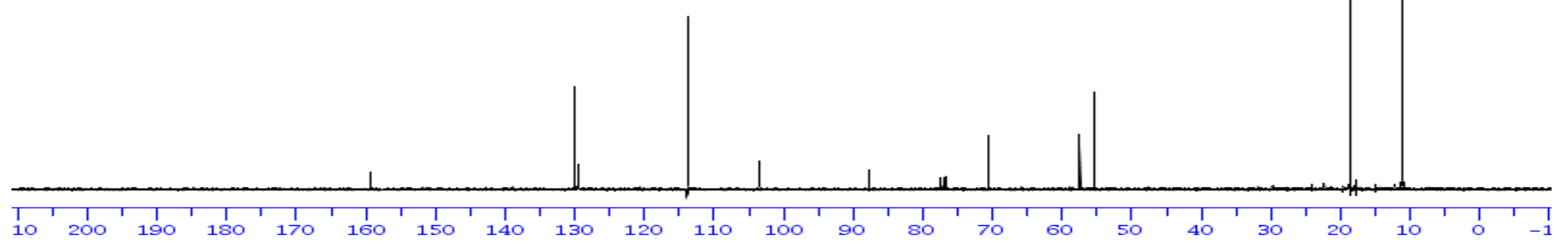




$$
\mathrm{Me}_{3} \mathrm{Si}={ }^{13} \mathrm{Si}^{i} \mathrm{Pr}_{3}
$$

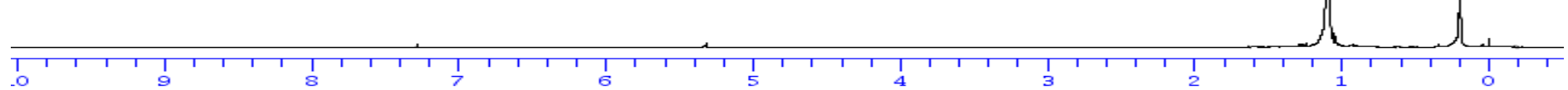

solvent: $\langle C D C I 3\rangle$

Frequency. $100.612769 \mathrm{MHz}$

$$
\mathrm{Me}_{3} \mathrm{Si}={ }^{13} \mathrm{Si}^{i} \mathrm{Pr}_{3}
$$

$15^{\prime}$

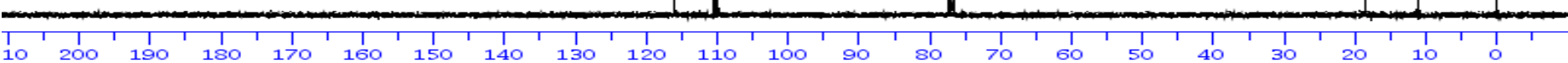

solvent: $\langle\mathrm{CDCI} 3\rangle$

Frequency. $100.61 \mathrm{MHz}$

$\mathrm{Me}_{3} \mathrm{Si}=\mathrm{Si}^{i} \mathrm{Pr}_{3}$

15

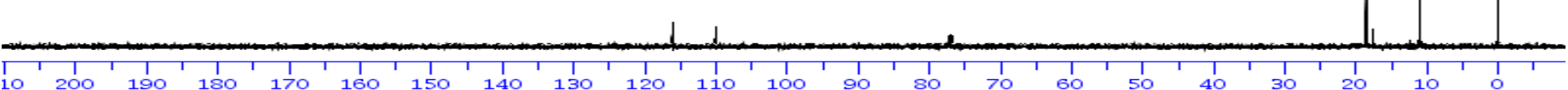




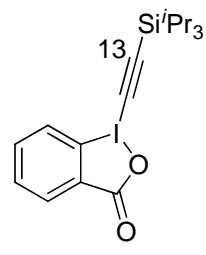

3d'

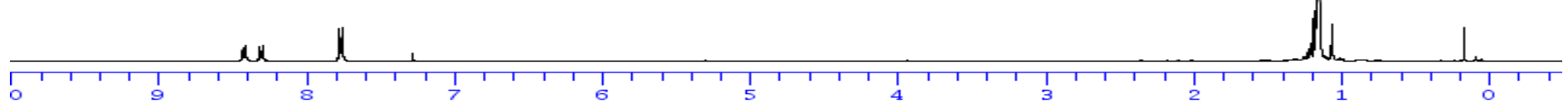

solvent: $\langle C D C I 3\rangle$

Frequency. $100.612769 \mathrm{MHz}$
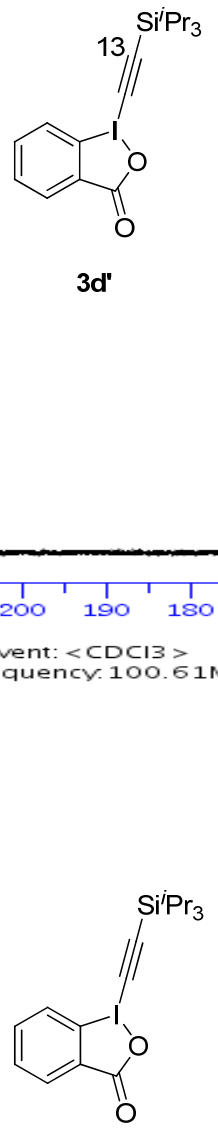

3d

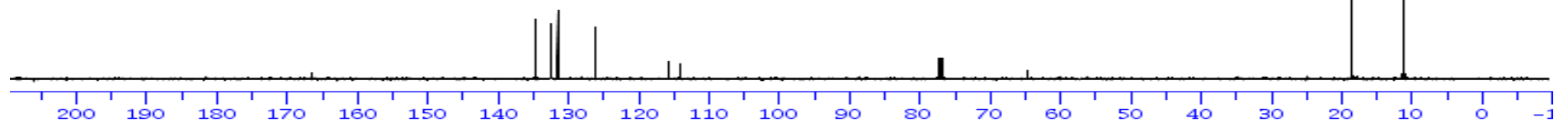




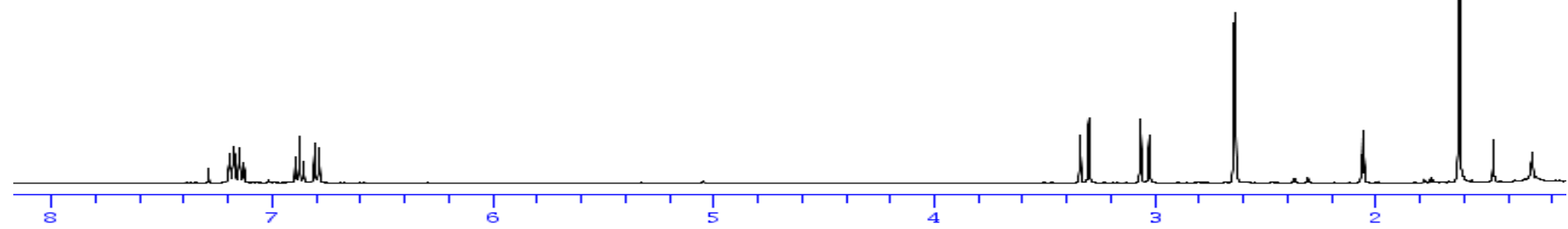
solvent: $\langle\mathrm{CDCI} 3\rangle$ Frequency. 100.61MHz<smiles>CC1(CC#C[AsH2+][SnH3])Cc2ccccc2O1</smiles>

$4 b^{\prime}$

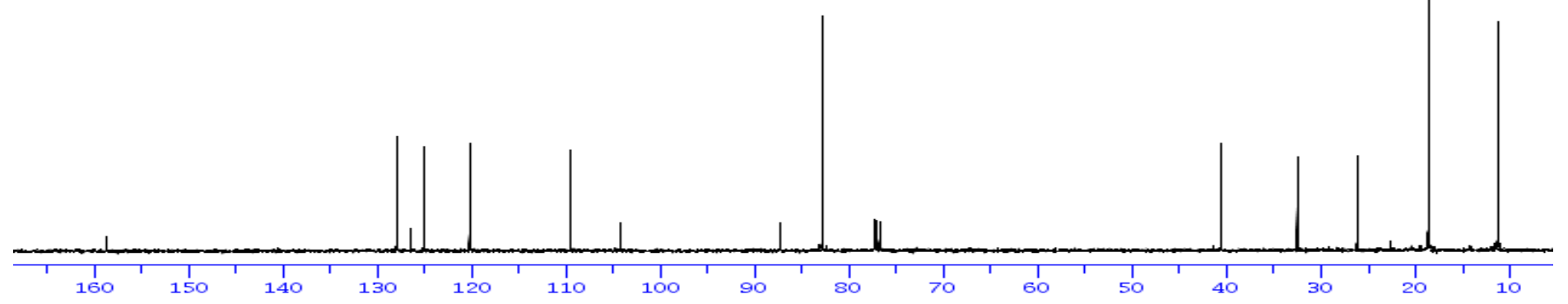
solvent: <CDCI3 > Frequency. $100.61 \mathrm{MHz}$

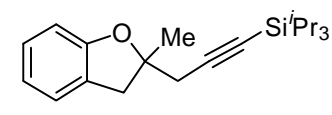

$4 b$

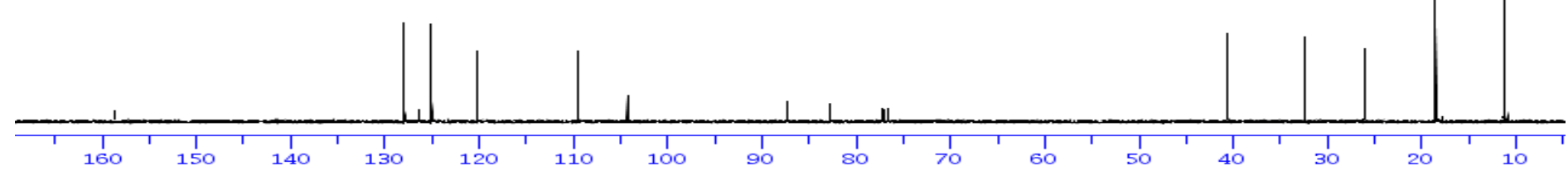


solvent: $\langle\mathrm{CDCI} 3\rangle$

Frequency. $100.61 \mathrm{MHz}$
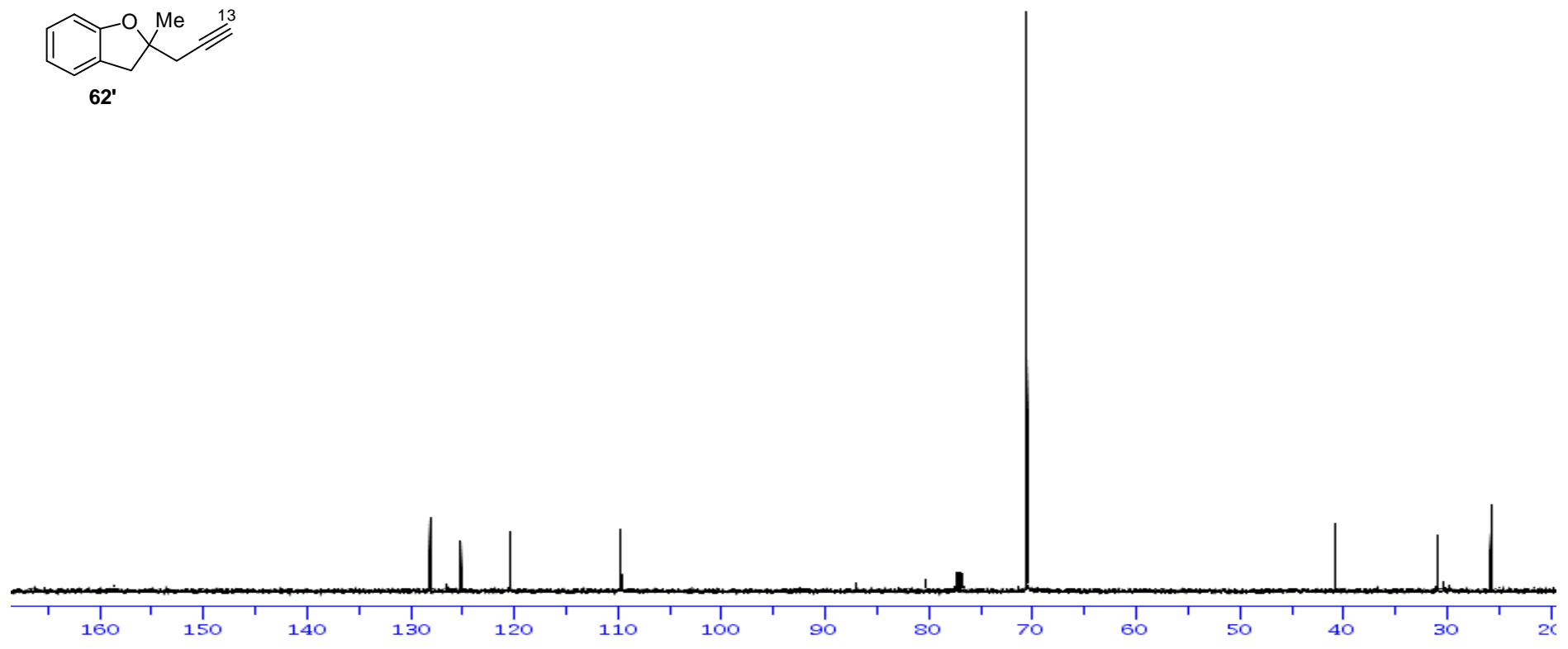

solvent: <CDCI3 >

Frequency. $100.61 \mathrm{MHz}$

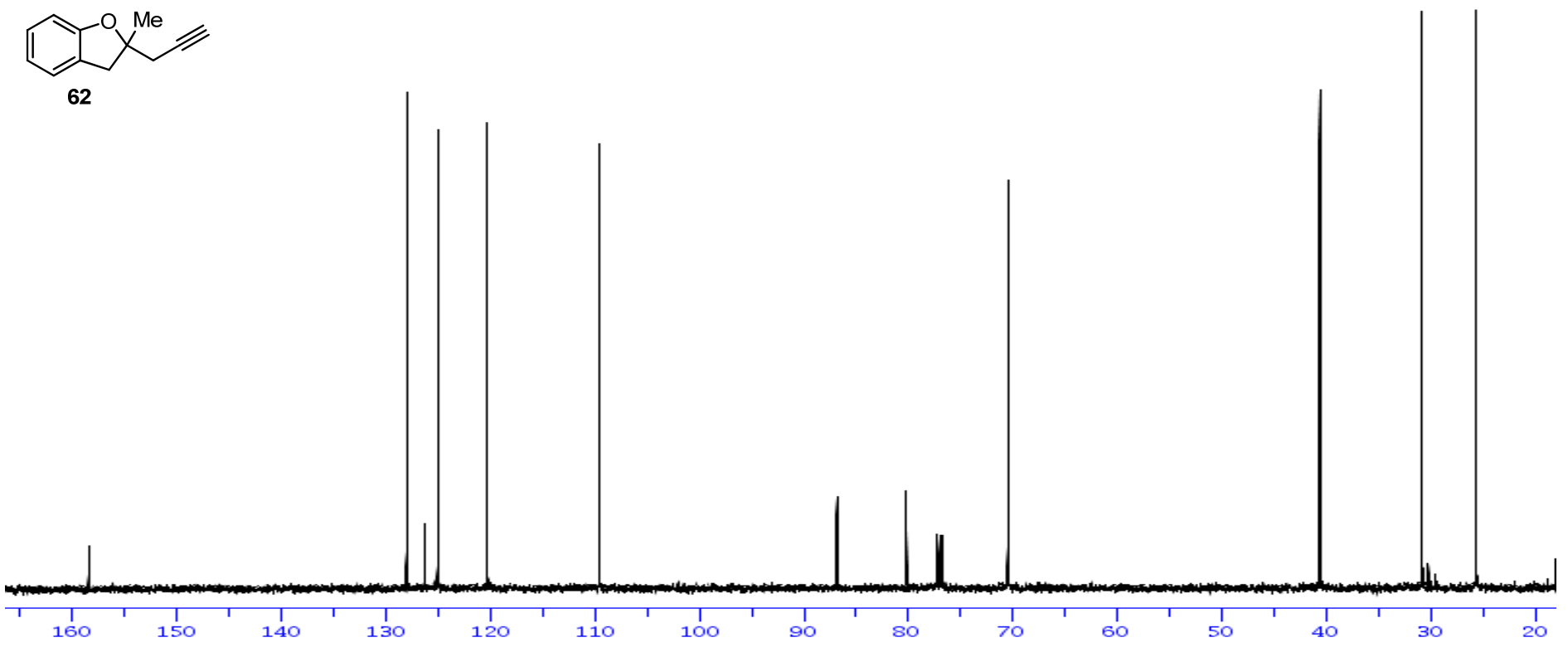

S79 
$13 C-N M R$

solvent: $<\mathrm{CDCI} 3>$

Frequency. 100.61MHz

$\mathrm{N}^{\mathrm{Me}}$

62

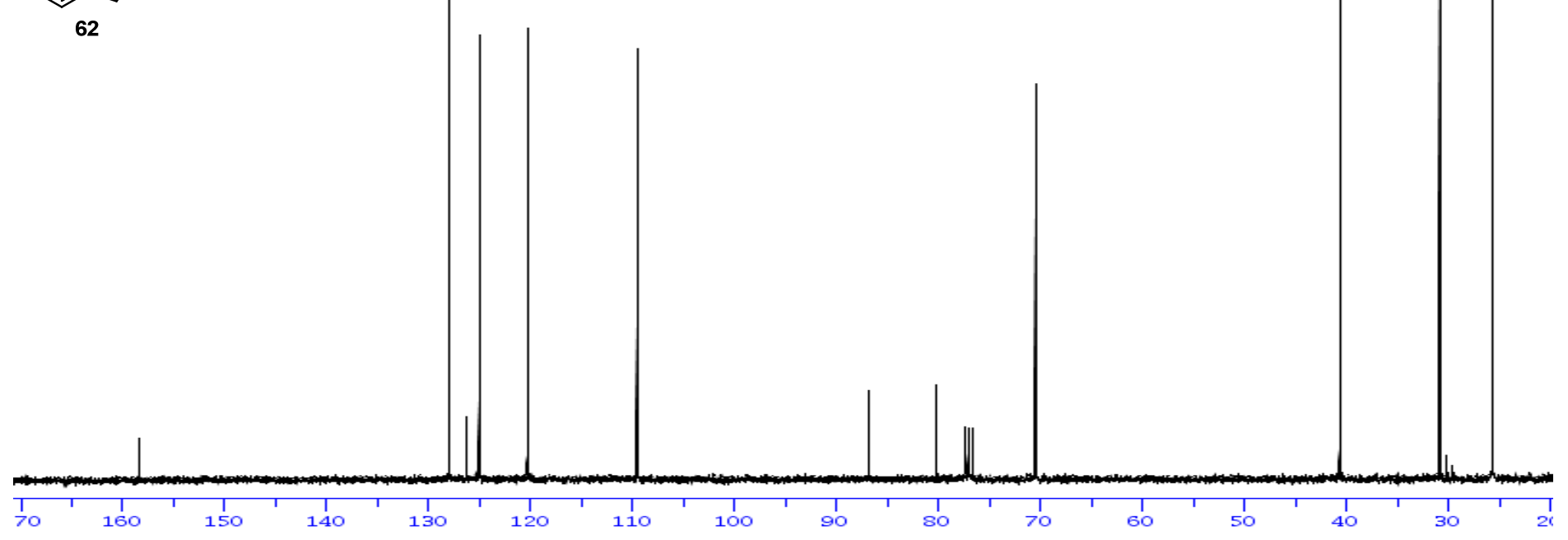

DEPT -90

solvent: $\langle\mathrm{CDCI} 3\rangle$

Frequency. 100.61 $\mathrm{MHz}$

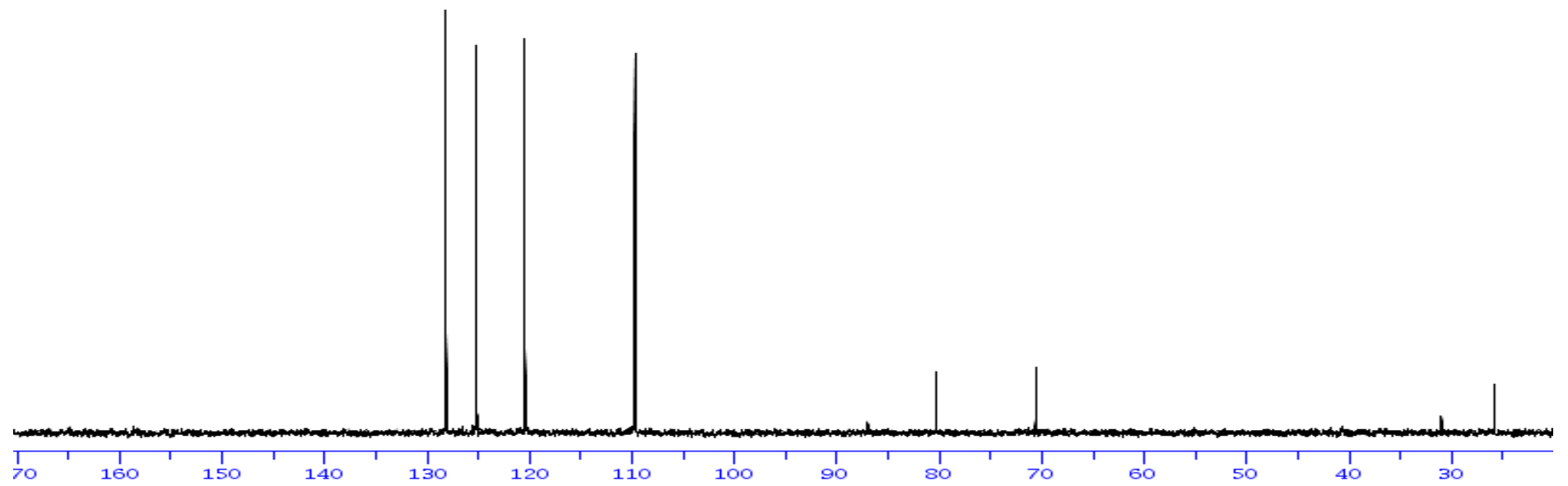

DEPT -135

solvent: $\langle C D C I 3>$

Frequency $10061 \mathrm{MHz}$

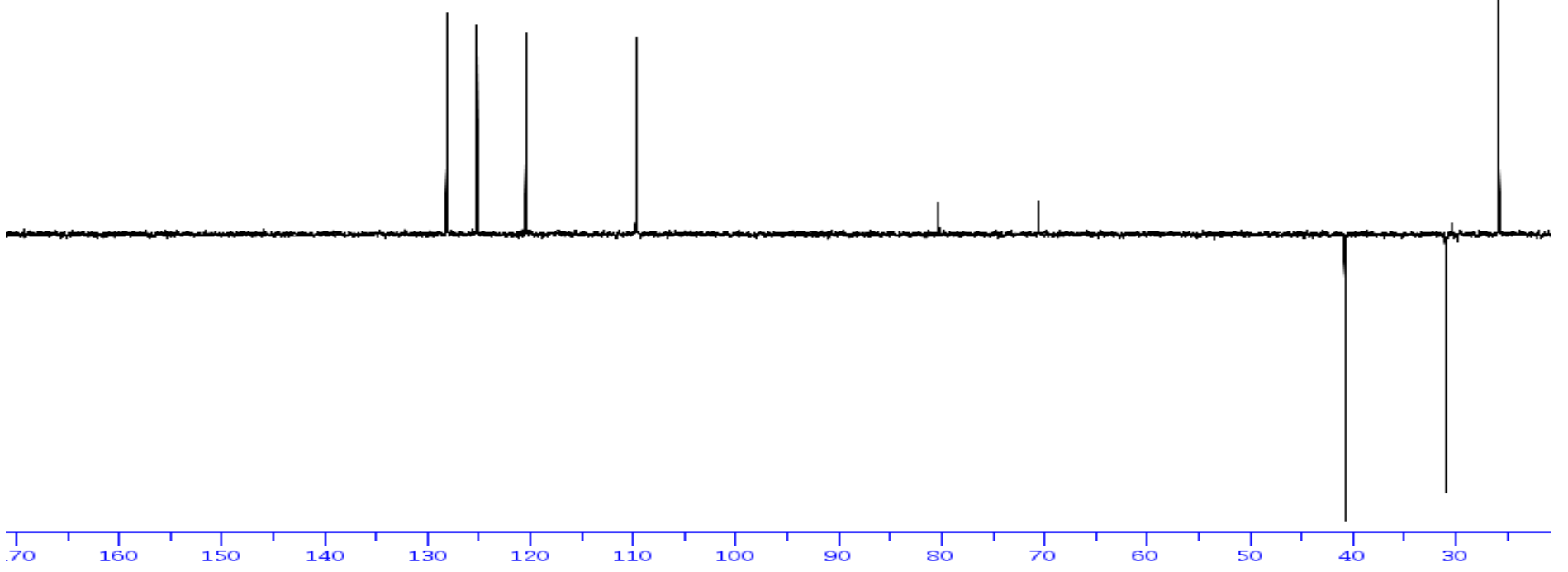



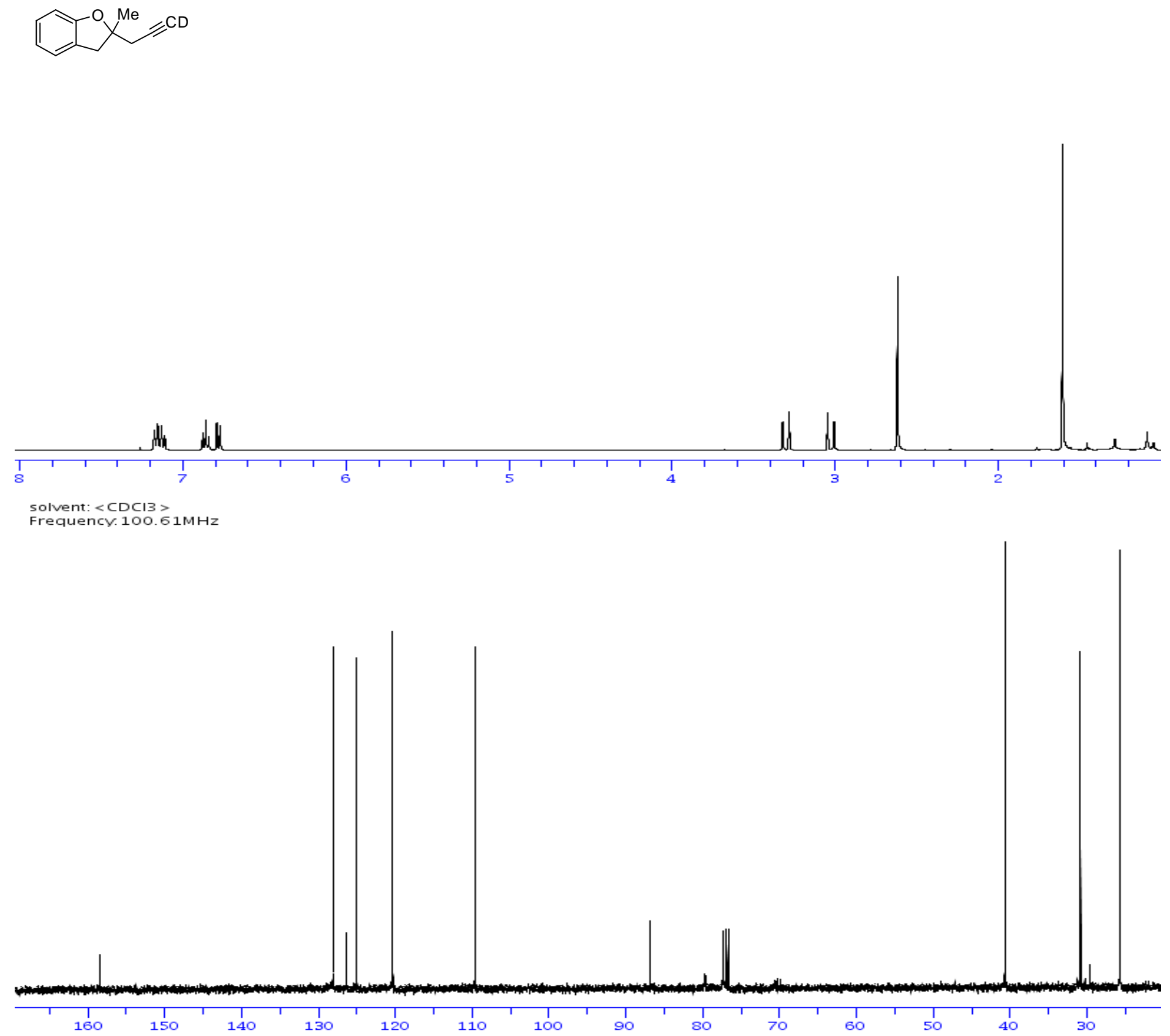